\title{
Magnetic Signature Control Strategies for an Unmanned Aircraft System
}

by

\section{Robert W. Forrester}

\author{
A Thesis submitted to \\ the Faculty of Graduate Studies and Research \\ in partial fulfilment of \\ the requirements for the degree of \\ Master of Applied Science \\ in
}

Aerospace Engineering

\author{
Ottawa-Carleton Institute for \\ Mechanical and Aerospace Engineering \\ Department of Mechanical and Aerospace Engineering \\ Carleton University \\ Ottawa, Ontario, Canada
}

September 2011(C)

Robert W. Forrester 
Library and Archives

Canada

Published Heritage

Branch

395 Wellington Street

Ottawa ON K1A ON4

Canada
Bibliothèque et

Archives Canada

Direction du

Patrimoine de l'édition

395 , rue Wellington

Ottawa ON K1A ON4

Canada
Your file Votre référence

ISBN: 978-0-494-83042-0

Our file Notre référence

ISBN: $978-0-494-83042-0$

\section{NOTICE:}

The author has granted a nonexclusive license allowing Library and Archives Canada to reproduce, publish, archive, preserve, conserve, communicate to the public by telecommunication or on the Internet, loan, distribute and sell theses worldwide, for commercial or noncommercial purposes, in microform, paper, electronic and/or any other formats.

The author retains copyright ownership and moral rights in this thesis. Neither the thesis nor substantial extracts from it may be printed or otherwise reproduced without the author's permission.

\section{AVIS:}

L'auteur a accordé une licence non exclusive permettant à la Bibliothèque et Archives Canada de reproduire, publier, archiver, sauvegarder, conserver, transmettre au public par télécommunication ou par l'Internet, prêter, distribuer et vendre des thèses partout dans le monde, à des fins commerciales ou autres, sur support microforme, papier, électronique et/ou autres formats.

L'auteur conserve la propriété du droit d'auteur et des droits moraux qui protège cette thèse. $\mathrm{Ni}$ la thèse ni des extraits substantiels de celle-ci ne doivent être imprimés ou autrement reproduits sans son autorisation.
In compliance with the Canadian Privacy Act some supporting forms may have been removed from this thesis.

While these forms may be included in the document page count, their removal does not represent any loss of content from the thesis.
Conformément à la loi canadienne sur la protection de la vie privée, quelques formulaires secondaires ont été enlevés de cette thèse.

Bien que ces formulaires aient inclus dans la pagination, il n'y aura aucun contenu manquant. 
The undersigned recommend to the Faculty of Graduate Studies and Research acceptance of the Thesis

\title{
Magnetic Signature Control Strategies for an Unmanned Aircraft System
}

\author{
Submitted by \\ Robert W. Forrester \\ in partial fulfilment of the requirements for the degree of \\ Master of Applied Science
}

M. Ahmadi, Thesis Co-supervisor

P. Straznicky, Thesis Co-supervisor

M. Yaras, Department Chair

Carleton University

September 2011 


\section{Abstract}

This thesis presents strategies for reducing the magnetic signature of an unmanned aircraft system (UAS). The GeoSurv II UAS is being designed by Carleton University for the purpose of conducting geomagnetic survey missions which requires the elimination of magnetic noise to ensure high quality survey data. A thorough experimental investigation on the GeoSurv II UAS was designed and performed to identify magnetic field sources and to create a metric of the magnetic signature of the current prototype. Two characterization schemes based on prior research, the Permanent Magnetic Dipole scheme (PMaD) and the Dual Permanent Magnetic Dipole scheme (DuPMaD), were developed to model simple magnetic field sources using magnetic point dipoles. Both PMaD and DuPMaD yielded nearly identical results, leading to the selection of the PMaD scheme for optimization and cancellation algorithms due to its simplicity.

A cancellation scheme, the Cancellation Magnet Configuration (CMC) strategy, was developed to reduce the far-field magnetic signature of a magnetic noise source through the use of a strategically configured permanent magnet. The CMC scheme was experimentally validated, yielding a $76.2 \%$ reduction in the magnetic noise of a servo actuator at a distance of $0.52 \mathrm{~m}$. Additionally, an optimization strategy, the Genetic Algorithm Magnetic Signature Optimization (GAMSO) strategy, was developed to strategically configure magnetic field sources to minimize the magnetic 
noise at wingtip magnetometer locations. The GAMSO strategy was experimentally validated, yielding a $98.8 \%$ and $85.8 \%$ magnetic noise reduction at the starboard and port wingtip locations, respectively, over the current configuration. Furthermore, the GAMSO solution exhibited small positional sensitivity, accounting for transient magnetic field changes due to small wingtip deflections experienced during flight. 


\section{Acknowledgments}

The completion of this research was accomplished with a great amount of support from individuals and organizations. I would like to thank my supervisors, Mojtaba Ahmadi and Paul Straznicky, for providing guidance and support to ensure that both my studies and my research remained in a constant state of progress. I would also like to thank Sander Geophysics Limited (SGL) for sponsoring this research. Specifically, I would like to thank Steve Ferguson, Francois Belanger, Matthew Wells, Lee Duncan, and Eric Renaud of SGL for all of their assistance and guidance with this research.

Additionally, I would like to thank Brad Nelson of the National Research Council for lending his expertise in magnetic signature control strategies. Much of the experimental testing in this report could not have been completed without Natural Resources Canada's Geomagnetic Laboratory; I would like to thank Lorne McKee, David Calp, and David Boteler for all of their cooperation. Additionally, former GeoSurv II undergraduate project students Shijun Thung, Jughyun Kim, Nilan Arthanayake, and Alexander Gale were all a tremendous help while conducting full scale testing at the Geomagnetic Laboratory in the middle of a July heat wave.

Finally, I would like to thank my parents, Colleen and Rob, and my wife, Taylor, for their constant encouragement and support. 


\section{Table of Contents}

Abstract

Acknowledgments $\quad$ v

Table of Contents vii

List of Tables $\quad$ xii

List of Figures $\quad$ xiv

Nomenclature $\quad$ xxi

1 Introduction 1

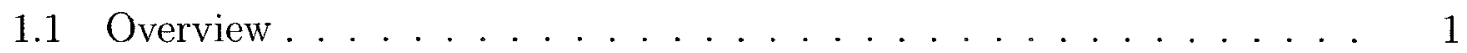

1.2 Research Motivation ..................... 2

1.2.1 Airborne Geomagnetic Surveys . . . . . . . . . . . . . . 2

1.2 .2 GeoSurv II UAS ................... 4

1.3 Research Objectives......................... 9

1.4 Research Contributions . . . . . . . . . . . . . . . . . . 11

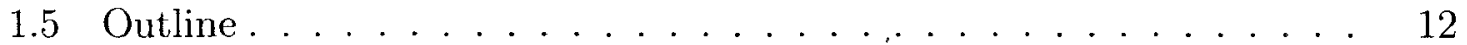

2 Magnetic Field Theory 15

2.1 Magnetic Flux Density and Magnetic Field Intensity . . . . . . . . 15 
2.2 Sources of Magnetic Fields . . . . . . . . . . . . . . . . . . 17

$2.2 .1 \quad$ Electromagnets . . . . . . . . . . . . . . . . . . . . 18

2.2 .2 Magnetic Materials . . . . . . . . . . . . . . 20

2.3 The Magnetic Dipole . . . . . . . . . . . . . . . . . . . . . . . . . 23

2.4 Geomagnetism . . . . . . . . . . . . . . . . . . 26

2.4 .1 Earth's Main Field . . . . . . . . . . . . . . . . . 26

2.4 .2 Diurnal Effects . . . . . . . . . . . . . . . 26

2.4 .3 Magnetic Anomalies . . . . . . . . . . . . . . 27

2.5 Literature Survey . . . . . . . . . . . . . . . . . . . . . 27

2.5.1 Dipole Modelling Strategies _. . . . . . . . . . . . . 28

2.5 .2 Testing Strategies . . . . . . . . . . . . . . 31

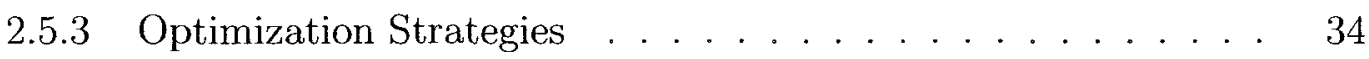

2.5 .4 Cancellation Strategies . . . . . . . . . . . . . 34

3 Measuring Magnetic Fields $\quad 37$

3.1 Introduction . . . . . . . . . . . . . . . . . . 37

3.2 Facilities for Magnetism Testing . . . . . . . . . . . . . . 37

3.3 GeoSurv II Magnetic Measurement System . . . . . . . . . . . . 45

3.3.1 Optically Pumped Cesium Magnetometer . . . . . . . . . 45

3.3.2 Fluxgate (Saturable-Core) Magnetometer . . . . . . . . . . 47

3.3 .3 Sensors Used for Testing . . . . . . . . . . . . . . . . . . 48

4 Testing and Analysis of Components of GeoSurv II Prototype 50

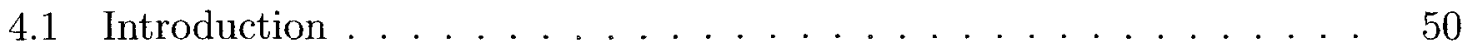

4.2 Experimental Procedure and Analysis Methods . . . . . . . . . 50

4.2 .1 Magnetic Component Localization . . . . . . . . . . . . 51

4.2 .2 Rotational Testing Procedure $\ldots \ldots \ldots \ldots \ldots$

4.2 .3 Gradiometer Testing Procedure . . . . . . . . . . . 53 
4.2 .4 Analysis Methods . . . . . . . . . . . . . . . . . 55

4.3 Servo Actuator Testing . . . . . . . . . . . . . . . . . 57

4.3.1 Determination of Rate of Decay of Magnetic Field . . . . . 58

4.3.2 Static Field Variations for Servos Under Rotation . . . . . . 61

4.3 .3 Powered Servo Testing . . . . . . . . . . . . . . 70

4.4 Engine Testing . . . . . . . . . . . . 76

4.4 .1 Gradiometer Test . . . . . . . . . . . . . . 76

4.4 .2 Powered Engine Testing . . . . . . . . . . . . 84

4.5 Avionics Testing . . . . . . . . . . . . . . . . 90

4.6 Component Testing Conclusions . . . . . . . . . . . . 95

$\begin{array}{lll}5 & \text { Characterization Strategies } & 97\end{array}$

5.1 Introduction . . . . . . . . . . . . . . . . . . 97

5.2 Requirement for an Improved Characterization Scheme . . . . . . . 97

5.3 Dual Permanent Magnetic Dipole Model . . . . . . . . . . . . 107

5.4 Permanent Magnetic Dipole Model . . . . . . . . . . . . . . . . 114

5.5 Validation using Finite Element Method Analysis . . . . . . . . . 119

5.6 Modelling Complex Magnetic Sources . . . . . . . . . . . . . . 122

5.7 Characterization Conclusions . . . . . . . . . . . . . 123

6 CMC Passive Cancellation Strategy 125

6.1 Introduction . . . . . . . . . . . . . . . . . 125

6.2 Development of Passive Cancellation Strategy . . . . . . . . 125

6.2 .1 Results from CMC Implementation . . . . . . . . . . . 128

6.3 Cancellation Conclusions . . . . . . . . . . . . . . . . . . . 139

7 Testing and Analysis of Assembled GeoSurv II Prototype 140

7.1 Introduction . . . . . . . . . . . . . 140 
7.2 Actuation of Control Surfaces . . . . . . . . . . . . . . . 141

7.3 Simulated Wingtip Deflection . . . . . . . . . . . . . 144

7.4 Gradiometer Test . . . . . . . . . . . . . . . . . . 146

7.5 Heading Test . . . . . . . . . . . . . . . . . . 149

7.6 Full-scale Testing Conclusions . . . . . . . . . . . . . . . 153

8 Genetic Algorithm Magnetic Signature Optimization 154

8.1 Introduction . . . . . . . . . . . . . . . . . . 154

8.2 Genetic Algorithm Theory and GAMSO Implementation $\ldots . . . .155$

8.2 .1 Constraints . . . . . . . . . . . . . . . . 158

8.2 .2 Fitness Function . . . . . . . . . . . . . . 162

8.2 .3 Population Creation $\ldots \ldots \ldots \ldots \ldots$

8.2 .4 Population Selection $\ldots \ldots \ldots$. . . . . . . . . 169

8.2 .5 Population Crossover . . . . . . . . . . . . . . . . 172

8.2 .6 Population Mutation . . . . . . . . . . . . . . 173

8.3 GAMSO Results . . . . . . . . . . . . . . . 173

8.3 .1 GAMSO Efficiency . . . . . . . . . . . . . . 174

8.3.2 GAMSO MATLAB Results . . . . . . . . . . . . 175

8.3.3 GAMSO Experimental Validation . . . . . . . . . . . 182

8.4 GAMSO Conclusions . . . . . . . . . . . . . . . . . . . 186

9 Conclusions and Recommendations 188

9.1 Summary . . . . . . . . . . . . . . . . . . . . . 188

9.2 Conclusions . . . . . . . . . . . . . . . . . . . . . . 190

9.3 Recommendations . . . . . . . . . . . . . . . . . . . . . . . . 191

$9.3 .1 \quad$ Future Work . . . . . . . . . . . . . . . . . . . . . 193

$\begin{array}{ll}\text { List of References } & 195\end{array}$ 
Appendix A Component Testing Figures

Appendix B Full-Scale UAS Testing Figures

Appendix C GAMSO Efficiency Investigation 


\section{List of Tables}

3.1 List of distances at which some common objects will generate a magnetic field of $1 \mathrm{nT} \ldots \ldots \ldots \ldots$

5.1 Comparison of the mean absolute error for the MoDEM and MoDEM2 characterization schemes ............................ 106

5.2 Model definition comparison for the MoDEM and DuPMaD nodels . 109

5.3 Comparison of the mean absolute error for the DuPMaD. MoDEM, and MoDEM2 characterization schemes . . . . . . . . 110

5.4 Comparison of the mean absolute error for the DuPMaD and PMaD

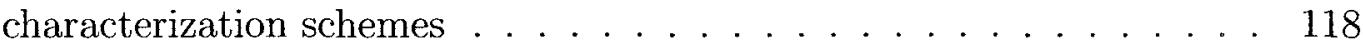

6.1 Comparison of the percentage reduction in the magnetic signature of the Hitec HSR-5990TG servo as a result of utilizing the CMC strategy

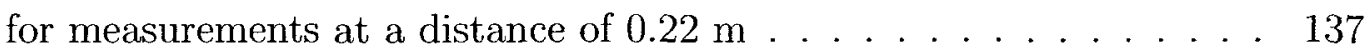

6.2 Comparison of the percentage reduction in the magnetic signature of the Hitec HSR-5990TG servo as a result of utilizing the CMC strategy for measurements at a distance of $0.33 \mathrm{~m}$. . . . . . . . . . . . 137

6.3 Comparison of the percentage reduction in the magnetic signature of the Hitec HSR-5990TG servo as a result of utilizing the CMC strategy for measurements at a distance of $0.52 \mathrm{~m} \ldots \ldots . \ldots 138$

8.1 Brief description of configurations used during optimization . . . . . . 168

8.2 Comparison of theoretical results for the GAMSO algorithm . . . . 176 
8.3 Comparison of theoretical and experimental results for the GAMSO algorithm . . . . . . . . . . . . . . . . 186

C.1 Number of generations and elapsed time required to reach global minimum with GAMSO . . . . . . . . . . . . . . 212 


\section{List of Figures}

1.1 Typical geomagnetic survey aircraft ................ . . 3

1.2 Orthogonal views of the current GeoSurv II Prototype configuration . 5

1.3 GeoSurv II prototype servo placement . . . . . . . . . . . . 7

2.1 Basic electromagnet . . . . . . . . . . . . . . 18

2.2 Basic hysteresis loop for a magnetic material . . . . . . . . . . . . 22

2.3 Comparison of dipole and quadrupole flux lines . . . . . . . . . . . 24

2.4 Behaviour of total magnetic field for dipole rotating about some fixed

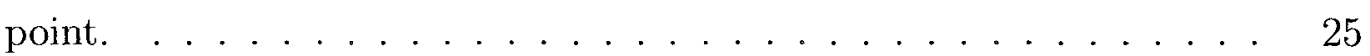

2.5 Dipole source modelled using Wells' MoDEM model . . . . . . . . . . 30

2.6 Experimental apparatus used by Versteeg et al. . . . . . . . . . . . 32

2.7 Effect of Mu-metal shield on Hitec HSM-7990TG servo . . . . . . . . 36

3.1 Magnetic field behaviour in VS3219 over the course of a day . . . . . 41

3.2 Magnetic field behaviour in ME2180 over the course of a day . . . . . 42

3.3 Magnetic field behaviour at SGL Headquarters over the course of a day 43

3.4 Absolute building and surrounding pathways at the Geomagnetic Lab-

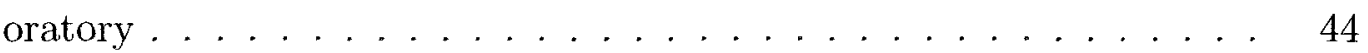

3.5 Magnetic field behaviour at the Geomagnetic Laboratory over the course of a day ....................... . . . . 45

3.6 Geometrics G-822A optically pumped Cesium magnetometer . . . . . 46

3.7 Billingsley TFM100 triaxial fluxgate magnetometer . . . . . . . . 48 
3.8 Sensitivity analysis for sensor hold time . . . . . . . . . . . . 49

4.1 Illustration of rotation test used during testing . . . . . . . . . 53

4.2 Illustration of gradiometer test used during testing $\ldots \ldots \ldots 55$

4.3 Hitec HSR-5990TG servo configurations used during testing . . . . 58

4.4 Magnetic field rate of decay results for different Hitec HSR-5990TG servo configurations . ....................... 60

4.5 MMTA-1D test apparatus with servo . . . . . . . . . . . 62

4.6 MMTA-3D test apparatus with servo in different configurations . . 64

4.7 Magnetic field vs rotation for a single Hitec HSR-5990TG servo collected using MMTA-1D . . . . . . . . . . . . . . . . 65

4.8 Magnetic field vs. rotation for a single Hitec HSR-5990TG servo collected using MMTA-3D . . . . . . . . . . . . . . . 66

4.9 Magnetic field vs. rotation for a single Hitec HSM-7990TG servo collected using MMTA-3D . . . . . . . . . . . . . . . 67

4.10 Comparison of magnetic field vs. rotation for three different Hitec HSR-5990TG servo configurations . . . . . . . . . . . . 68

4.11 Variability in the magnetic signatures of 13 Hitec HSR-5990TG servos 70

4.12 Behaviour of Magnetic Field while Powering a single Hitec HSR5990 TG servo . . . . . . . . . . . . . . . . . . 72

4.13 Behaviour of Magnetic Field while actuating a single Hitec HSR$5990 \mathrm{TG}$ servo through its motion range . . . . . . . . . . . . .

4.14 Behaviour of Magnetic Field while manually actuating a single Hitec HSR-5990TG servo through its motion range . . . . . . . . . . 74

4.15 Transient effect of cycling a Hitec HSR-5990TG servo at high load . . 75

4.16 Experimental setup used during engine gradiometer testing . . . . . 77

4.17 Behaviour of magnetic field for the GeoSurv II fuselage/engine moving past two fluxgates in a gradiometer configuration . . . . . . 78 
4.18 Behaviour of magnetic field for the GeoSurv II fuselage/engine moving past two Cesium magnetometers in a gradiometer configuration . .

4.19 Transient magnetic noise profile for each sensor for the GeoSurv II fuselage/engine moving past two fluxgate magnetometers in a gradiometer configuration while manually rotating the propeller . . . . . . .

4.20 Amplitude of the transient magnetic field for the GeoSurv II fuselage/engine moving past two fluxgate magnetometers in a gradiometer configuration while manually rotating the propeller . . . . . . . 82

4.21 Amplitude of the transient magnetic field for the GeoSurv II fuselage/engine moving past two Cesium magnetometers in a gradiometer configuration while manually rotating the propeller . . . . . . . 83

4.22 Experimental setup used for the engine run . . . . . . . . . . . . . 84

4.23 Magnetic field behaviour while manually rotating propeller to simulate a running engine with magnetometers at wingtip spacing . . . . .

4.24 Magnetic noise versus frequency in the 0 to $80 \mathrm{~Hz}$ range for the GeoSurv II engine operating at an average speed of 2140 RPM $(35.7 \mathrm{~Hz})$. . .

4.25 Magnetic noise versus frequency in the 0 to $5 \mathrm{~Hz}$ range for the GeoSurv II engine operating at an average speed of 2140 RPM $(35.7 \mathrm{~Hz}) \ldots . \quad 88$

4.26 Power spectral density analysis of magnetic noise for the GeoSurv II engine operating at an average speed of 2140 RPM $(35.7 \mathrm{~Hz}) \ldots$. . . 89

4.27 Experimental setup used during flight avionics gradiometer testing . . 90

4.28 Behaviour of magnetic field for the flight avionics moving longitudinally between two fluxgate magnetometers in a gradiometer configuration .

4.29 Behaviour of magnetic field for the flight avionics moving longitudinally between two Cesium magnetometers in a gradiometer configuration .

4.30 Behaviour of magnetic field for the flight avionics moving laterally between two fluxgate magnetometers in a gradiometer configuration . . 
4.31 Behaviour of magnetic field for the flight avionics moving laterally between two Cesium magnetometers in a gradiometer configuration . . . 95

5.1 MoDEM characterization scheme with fitness function specified by Wells $[1] \ldots \ldots \ldots . \ldots . \ldots . \ldots . \ldots . \ldots 9$

5.2 Effect of the interference vector interacting with the background field 100

5.3 MoDEM2 characterization scheme with fitness function defined in this

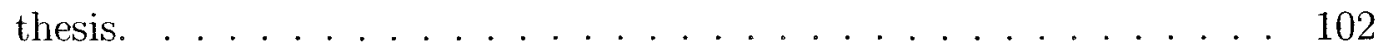

5.4 MoDEM characterization compared to experimental data collected in multiple measurement planes . . . . . . . . . . . . . . . 104

5.5 MoDEM2 characterization compared to experimental data collected in multiple measurement planes . . . . . . . . . . . . . 105

5.6 Geometry used for DuPMaD characterization . . . . . . . . . 108

5.7 DuPMaD characterization of Hitec HSR-5990TG servo. . . . . . . . 111

5.8 DuPMaD characterization of Hitec HSM-7990TG servo. . . . . . . . 112

5.9 Comparison of MoDEM2 and DuPMaD characterization schemes for the Hitec HSR-5990TG servo. . . . . . . . . . . . . . . 114

5.10 PMaD characterization of Hitec HSR-5990TG servo. . . . . . . . 116

5.11 PMaD characterization of Hitec HSM-7990TG servo. . . . . . . . . 117

5.12 Illustration of magnetic dipole location and orientation within the Hitec HSR-5990TG servo casing . . . . . . . . . . . . . . . . 119

5.13 Comparison between experimental data and theoretical results produced by MATLAB and COMSOL for the DuPMaD and PMaD characterization schemes ................... 121

5.14 Characterization of engine using four permanent magnetic dipoles . . 122

6.1 Direction of magnetic moment vector for permanent magnet used in CMC strategy . . . . . . . . . . . . . . . . 126 
6.2 Magnet mount constructed to hold cancellation magnet for the Hitec HSR-5990TG servo . . . . . . . . . . . . . . . . . 129

6.3 Experimental setup used for CMC validation . . . . . . . . . 129

6.4 Theoretical CMC results for the Hitec HSR-5990TG servo at a distance of $0.22 \mathrm{~m} \ldots \ldots \ldots \ldots \ldots \ldots$

6.5 Theoretical CMC results for the Hitec HSR-5990TG servo at a distance of $0.33 \mathrm{~m} \ldots \ldots \ldots \ldots \ldots 131$

6.6 Theoretical CMC results for the Hitec HSR-5990TG servo at a distance

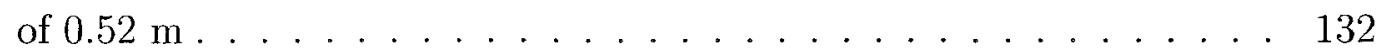

6.7 Experimental CMC results for the Hitec HSR-5990TG servo at a distance of $0.22 \mathrm{~m} \ldots \ldots \ldots \ldots \ldots$

6.8 Experimental CMC results for the Hitec HSR-5990TG servo at a dis-

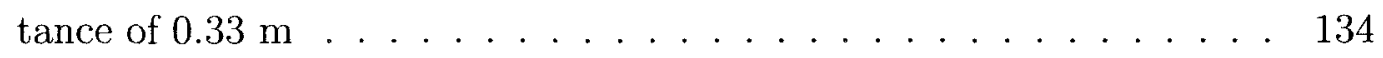

6.9 Experimental CMC results for the Hitec HSR-5990TG servo at a distance of $0.52 \mathrm{~m} \ldots \ldots \ldots \ldots \ldots \ldots$

7.1 Experimental setup used for indoor full scale testing . . . . . . . . . . 142

7.2 Behariour of Magnetic Field while actuating both flaperons in a roll

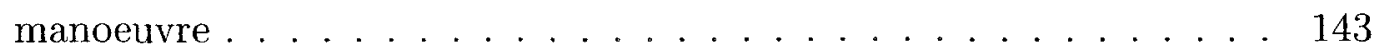

7.3 Analysis of flaperon magnetic noise in the frequency domain . . . . . 144

7.4 Behaviour of wingtip magnetic noise while simulating wingtip deflections 146

7.5 Experimental setup used for full scale gradiometer testing . . . . . . . 147

7.6 Behaviour of magnetic noise for GeoSurv II while moving past two Cesium Magnetometers in a gradiometer configuration . . . . . . . . 148

7.7 Behaviour of magnetic noise gradient for GeoSurv II while moving past two Cesium Magnetometers in a gradiometer configuration . . . . . . 149

7.8 Locations of measurement points used during full scale heading test . 150

7.9 Experimental setup used during full scale heading test . . . . . . . 151 
7.10 Behaviour of wingtip magnetic noise for GeoSurv II at various headings 152

8.1 Block diagram of the processes used in genetic algorithms . . . . . . 157

8.2 GeoSurv II coordinate system used for GAMSO strategy . . . . . . . 161

8.3 Illustration of servo locations and orientations for current and GAMSO optimized configurations . . . . . . . . . . . . . . 177

8.4 Magnetic field at wingtip magnetometers for the current servo config-

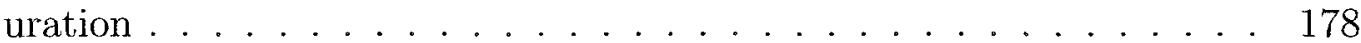

8.5 Magnetic field at wingtip magnetometers for the manually optimized

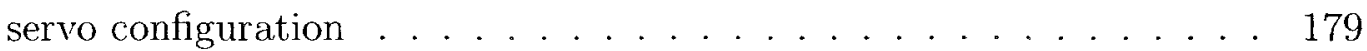

8.6 Magnetic field at wingtip magnetometers for the GAMSO optimized servo configuration ...................... . . . 180

8.7 Magnetic field contour in the xy-plane for current configuration . . . 181

8.8 Magnetic field contour in the xy-plane for GAMSO optimized config-

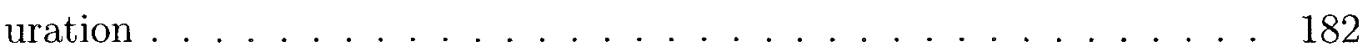

8.9 Current configuration used during GAMSO validation . . . . . . . . 183

8.10 GAMSO optimized configuration used during GAMSO validation . . 184

A.1 Magnetic field rate of decay results for a single Hitec HSR-5990TG servo200

A.2 Magnetic field rate of decay results for dual Hitec HSR-5990TG servos 201

A.3 Magnetic field rate of decay results for dual counteroriented Hitec HSR-

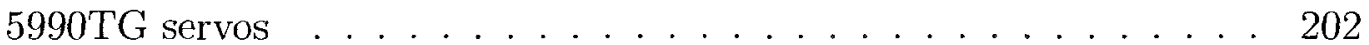

A.4 Magnetic field vs. rotation for dual Hitec HSR-5990TG servos collected

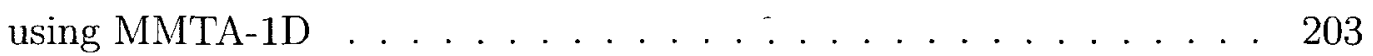

A.5 Magnetic field vs. rotation for dual counteroriented Hitec HSR5990TG servos collected using MMTA-1D . . . . . . . . . . . . 204

A.6 Transient effect of cycling a Hitec HSR-5990TG servo at low load . . 205

A.7 Transient effect of cycling a Hitec HSR-5990TG servo at medium load 206

B.1 Behaviour of magnetic field while actuating both elevators . . . . . 208 
B.2 Behaviour of magnetic field while actuating both rudders . . . . . 209

B.3 Behaviour of magnetic field while actuating the throttle . . . . . 210 


\section{Nomenclature}

\begin{tabular}{|c|c|c|}
\hline \multicolumn{3}{|c|}{ List of Symbols } \\
\hline Symbol & Description & Units \\
\hline$\vec{B}$ & Magnetic Flux Density & nanoTesla (nT) \\
\hline$\vec{H}$ & Magnetic Field Strength & ampere/metre $(\mathrm{A} / \mathrm{m})$ \\
\hline$M$ & Magnetization & ampere/meter $(\mathrm{A} / \mathrm{m})$ \\
\hline$\vec{m}$ & Magnetic Dipole Moment & ampere $\cdot$ metre $^{2}\left(\mathrm{~A} \cdot \mathrm{m}^{2}\right)$ \\
\hline$\mu$ & Magnetic Permeability & henry/metre $(\mathrm{H} / \mathrm{m})$ \\
\hline$\mu_{o}$ & Magnetic Permeability (vacuum) & henry/metre $(\mathrm{H} / \mathrm{m})$ \\
\hline$\mu_{r}$ & Relative Magnetic Permeability & $\frac{H / m}{H / m}$ \\
\hline$I$ & Current & ampere $(\mathrm{A})$ \\
\hline$\vec{r}$ & Position Vector & metre $(\mathrm{m})$ \\
\hline$\hat{r}$ & Unit Position Vector & $(\mathrm{m} / \mathrm{m})$ \\
\hline
\end{tabular}


List of Acronyms

\begin{tabular}{|c|c|}
\hline Acronym & Description \\
\hline $\mathrm{CMC}$ & Cancellation Magnet Configuration \\
\hline $\mathrm{DAQ}$ & Data Acquisition \\
\hline DDP & Direct Dipole Parameterization \\
\hline DuPMaD & Dual Permanent Magnet Dipole Model \\
\hline FFT & Fast Fourier Transform \\
\hline GAMSO & Genetic Algorithm Magnetic Signature Optimization \\
\hline MMTA & Magnetic Moment Test Apparatus \\
\hline MoDEM & Modified Dipole Ellipse Model \\
\hline $\mathrm{OFA}$ & Optimal Field Attenuation \\
\hline PMaD & Permanent Magnet Dipole Model \\
\hline PSD & Power Spectral Density \\
\hline RPM & Revolutions Per Minute \\
\hline SGL & Sander Geophysics Limited \\
\hline UAS & Unmanned Aircraft System \\
\hline UAV & Unmanned Aerial Vehicle \\
\hline UUV & Unmanned Underwater Vehicle \\
\hline
\end{tabular}




\section{Chapter 1}

\section{Introduction}

\subsection{Overview}

Airborne geomagnetic surveys are performed in order to determine the magnetic field over geological regions of interest. They require high resolution measurements of the magnetic field to identify local magnetic field variations, which can be used to indicate the presence of geological anomalies such as mineral or oil deposits. Due to the inherent properties of magnetic fields, the magnetic field measured during the survey may not necessarily be the true value of the earth-field at that survey point. Magnetic field sensors (magnetometers) measure the net magnetic field which means that if any magnetic interference is present, it will also be included in the measurement. Therefore, magnetic noise will compromise the accuracy and consistency of magnetic data.

Magnetometers can measure either the vector or total field depending on the type of magnetometer selected, and are unable to distinguish between fields that they are intended to measure such as the earth-field and those of magnetic interference sources. Interference generated from magnetic materials and low frequency $(<10 \mathrm{~Hz})$ magnetic sources most substantially affect survey data. Thus significant attention is 
given towards attenuating their effect at magnetometer locations on survey aircraft. Interference generated from magnetic fields at higher frequencies $(>10 \mathrm{~Hz})$ can be removed through filtering.

Geomagnetic survey aircraft are designed such that the magnetic interference at magnetometer locations is attenuated. This is generally accomplished by positioning the magnetometers far enough away from the airframe so that the magnetic interference generated by the aircraft does not affect the magnetometer readings. The current practice is also to replace ferromagnetic parts of the aircraft with non-ferromagnetic materials, which incurs cost and recertification of the aircraft for flight. To avoid having to modify airframes of as-built aircraft, purpose-built unmanned aircraft systems (UAS) may be utilized with the added advantage of requiring a smaller field crew and the ability to fly closer to the terrain. However. UAS's are smaller in size which implies increased proximity between magnetic noise sources and magnetometers. This thesis presents the development of a novel strategy to characterize, and attenuate the magnetic interference of an unmanned geomagnetic survey aircraft.

\subsection{Research Motivation}

\subsubsection{Airborne Geomagnetic Surveys}

Traditionally, airborne geomagnetic surveys are completed with manned fixed wing or rotary wing aircraft. This typically requires a field crew of one or two geophysicists, a pilot, a co-pilot, as well as an aircraft maintenance engineer (AME). A typical geomagnetic survey aircraft is shown in Figure 1.1. 


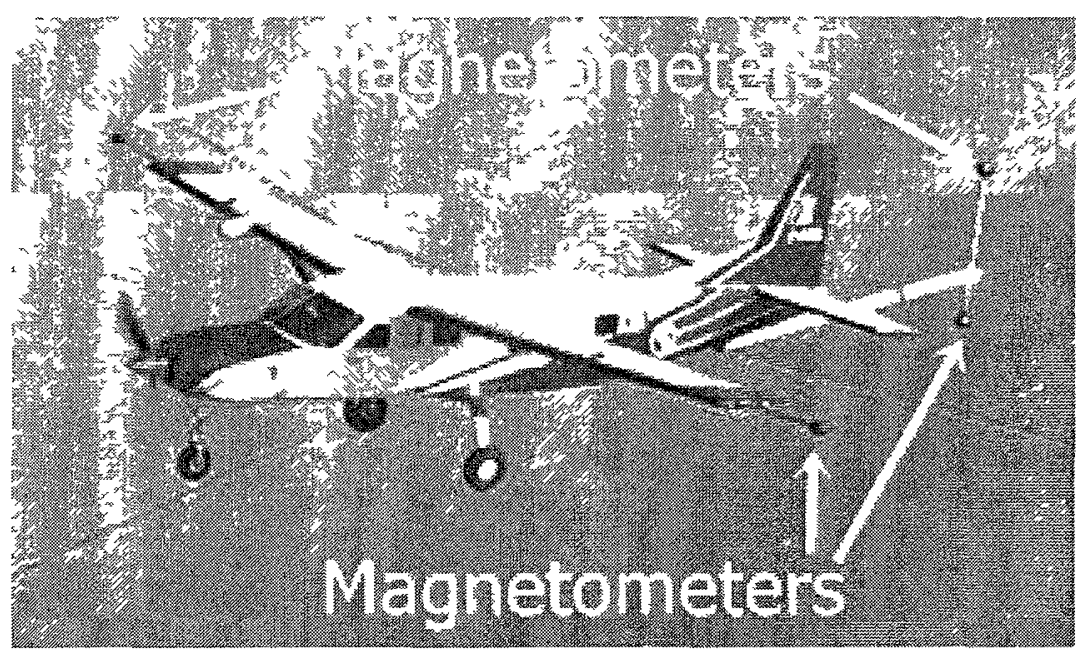

Figure 1.1: An SGL Cessna 208B Grand Caravan shown equipped for triaxial gradiometer surveying. Note the stingers used to separate the magnetometers from the airframe. Courtesy of Sander Geophysics Limited.

Ideally, geomagnetic surveys are flown "slow and low" meaning that it is desirable for the survey aircraft to have a low velocity and low altitude. This is desirable for several reasons:

- Flying slowly allows the aircraft to follow the drape more closely. The drape is the projection of the terrain at a constant elevation above the actual terrain. For example, if the terrain has a hill such that the altitude on the ground increases, then the drape will also increase in altıtude. By following the drape more closely, arrborne geomagnetic measurements can give a closer representation of magnetıc anomalies.

- Flymg slowly allows for more measurement points to be collected over a set distance. since the distance between measurement points will decrease with speed for a set measurement frequency.

- The magnitude of a magnetic field is proportional to the inverse cube of distance from source to measurement point, meaning that the closer the measurement 
point is to the source, the larger the measured field will be. Thus, flying at a lower altitude means that the magnetic field intensity will be greater, and hence the survey resolution will be increased. Therefore, anomalies will appear sharper in the data, again aiding data interpretation. Results from Caron [2] indicate that the advantage of flying lower altitude surveys disappears below elevations of $50 \mathrm{~m}$ for the survey area studied.

Manned aircraft cannot fly close to the terrain due to the safety concerns associated with having humans onboard. Comparatively, UAS's are able to fly much lower to the terrain since the safety requirements of unmanned aircraft are significantly less than their manned counterparts. Additionally, the use of UAS's allows for a reduction in the number of field crew members to as few as a single person. leading to a decrease in the resources needed to perform a geomagnetic survey and hence making UAS's more economical. Therefore, there are both economic and performance drivers toward using unmanned aircraft to perform the function of manned surveys.

However, since UAS's are typically smaller than manned aircraft, the high sensitivity magnetometers will be located much closer to onboard sources of magnetic interference relative to manned aircraft. Therefore, special attention must be given to ensuring that the magnetic interference at magnetometer locations is attenuated to the greatest extent possible.

\subsubsection{GeoSurv II UAS}

Carleton University is currently developing a UAS, known as GeoSurv II, capable of high resolution geomagnetic surveys. GeoSurv II has a wingspan of $4.88 \mathrm{~m}$, a length of $4.27 \mathrm{~m}$, and stands $0.91 \mathrm{~m}$ off the ground. The airframe is composed almost entirely of composite materials with the exception of some fasteners. The use of metals is 
kept to a minimum, and nonmagnetıc metals are used where necessary. At present, a prototype of GeoSurv II has been built and is being prepared for high-speed taxi tests and first flight The curnent weight of the prototype is $95.25 \mathrm{~kg}$. The GeoSurv II is designed to have a field crew of no more that 2 persons Refer to Figure 1.2 for a schematic of the current GeoSurv II configuration

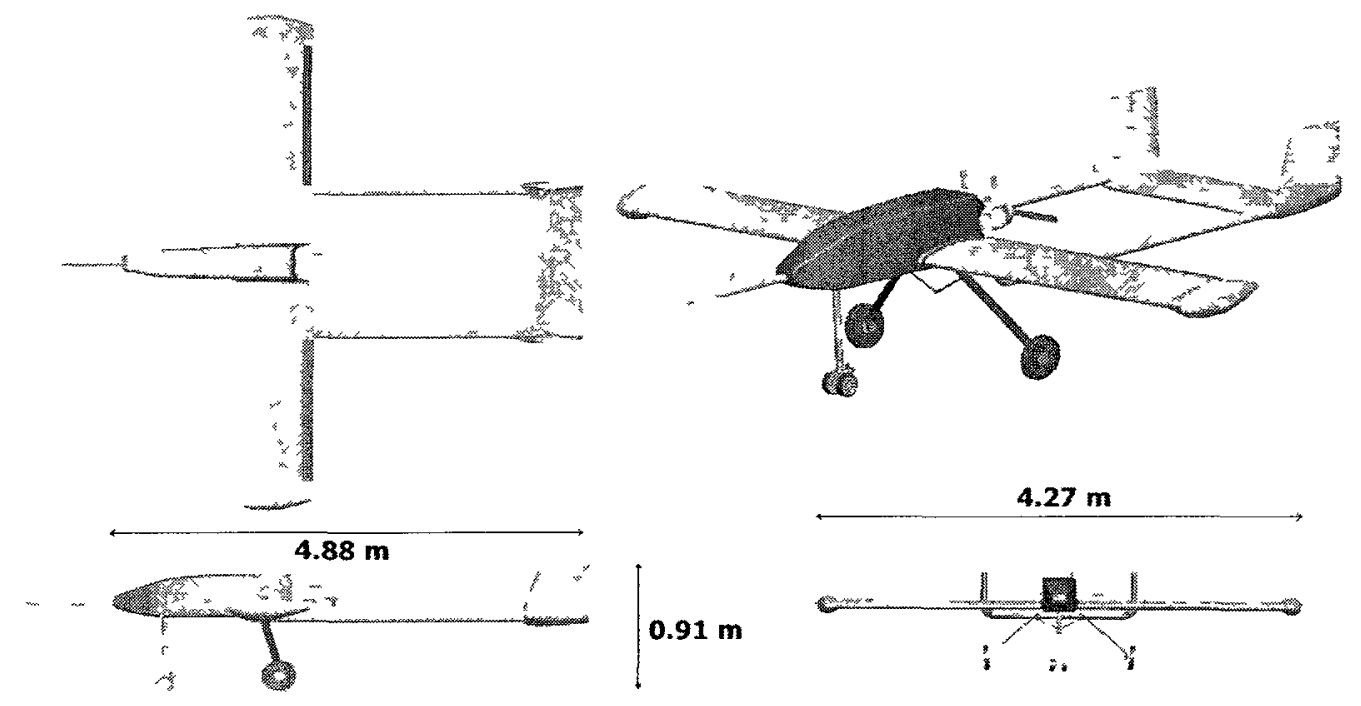

Figure 1.2: Orthogonal views of the current GeoSurv II Prototype configuration. Top (top left), side (bottom left), and front (bottom right) views are shown.

GeoSurv II is being developed in partnership with Sander Geophysics Limited (SGL), a world leader in airborne geophysical surveying, and geological and environmental mapping The project team is composed of the undergraduate design team and the graduate research team. The undergraduate design team is responsible for the design and integration of the aircraft structure and systems, and is composed of fourth year aerospace, mechanical, systems and computer engineering students. The graduate research team focuses on the development of technologies specific to geomagnetic survey UAS's and addresses five research topics - obstacle detection. autonomous operation, low cost composite airframes, geomagnetic data acquisition, 
and magnetic signature control - which are conducted by graduate students. Both project teams also include faculty members and industry professionals. This thesis presents the work completed on the magnetic signature control research project.

\section{Sources of Magnetic Noise}

GeoSurv II is designed to operate within the niche market of unmanned geomagnetic surveying. As such, the availability of magnetically quiet off-the-shelf components for the power plant and actuation is scarce, hence there are many sources of magnetic fields onboard GeoSurv II that will contribute to the magnetic interference at the wingtip magnetometers. Although the airframe is composed primarily of composite materials, metal fasteners were necessary for certain high load components. Mostly nonmagnetic materials have been selected for such fasteners. Additionally, engine components such as the crankshaft and connecting rods are made of high strength steel which is unfavourable for magnetic signature control. Furthermore, the final design of GeoSurv II will incorporate a generator, and additional avionics associated with the mission and autonomous operation which will likely increase the magnetic signature of the aircraft.

Most importantly, the actuation of the current GeoSurv II prototype is performed by electric servo actuators. The permanent magnets within the $\mathrm{DC}$ motor of each servo actuator significantly affect the magnetic signature of GeoSurv II. The locations of the servo actuators onboard the current GeoSurv II prototype, along with the locations of the engine and magnetometers are shown in Figure 1.3. 


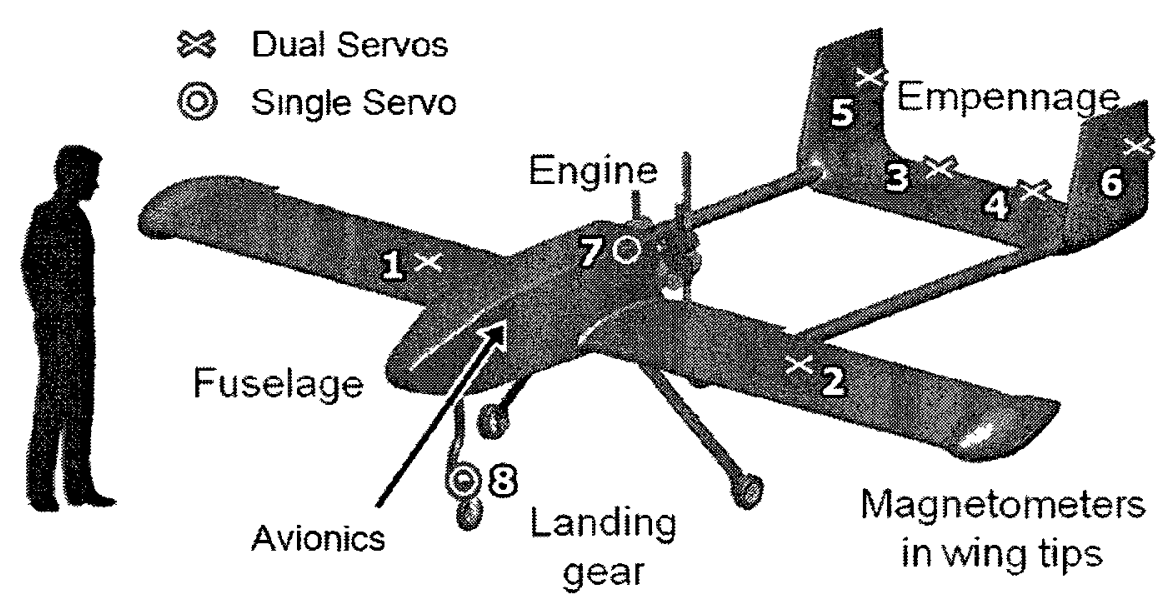

Figure 1.3: GeoSurv II prototype servo placement. There are 14 servos in total. Labels: (1) starboard flaperon servos. (2) port flaperon servos, (3) starboard elevator servos, (4) port elevator servos, (5) starboard rudder servos, (6) port rudder servos, (7) throttle servo, (8)nose landing gear servo. The flaperon servos are closest to the wingtip magnetometers, hence are responsible for the greatest contribution of magnetic noise.

\section{Magnetic Signature Requirements}

The magnetic signature requirements for GeoSurv II were assigned by SGL as outlined in [3]. The established requirements specify that the final GeoSurv II design be competitive in its performance with manned geomagnetic survey aircraft. The requirements related to the magnetic signature of GeoSurv II are:

- Requirement 3.1.1-3: Uncompensatable magnetic fields (noise) generated by the UAS shall not exceed 0.1 nanoTesla (nT) for fixed sources.

- Requirement 3.1.1-4: Uncompensatable magnetic fields (noise) generated by the UAS shall not exceed $0.01 \mathrm{nT}$ for time-varying sources, in a 0 to $5 \mathrm{~Hz}$ bandwidth.

- Requirement 3.1.1-9: The aircraft structure shall consist of only composite materials within $30 \mathrm{~cm}$ of the magnetometer sensors. Use of metals within this area shall be minimized and shall in any case be limited to nonmagnetic 
hardware components.

- Requirement 3.1.3-4: If 2 magnetometers are carried, these shall be mounted in horizontal gradiometer configuration.

- Requirement 3.1.3-5: The aircraft shall carry 1 or 2 G-822A magnetometer sensors.

- Requirement 3.1.3-6; The aircraft shall carry 1 Billingsley TFM 100 vector magnetometer sensor.

- Requirement 3.1.3-7: All materials within 1 foot (30 centimeters) of magnetometer(s) shall be non-conductive, with the exception of small nonmagnetic fasteners.

The magnetometers that SGL uses are the Geometrics G-822A optically pumped cesium magnetometers which have a sensitivity of $0.005 \mathrm{nT}$. The magnetometer data is then filtered using computer-controlled real-time digital compensation, which results in an overall system resolution of $0.01 \mathrm{nT}$. Since the sensors used by SGL in their current aircraft fleet are highly sensitive, any UAS completing geomagnetic surveys will need to have magnetic interference below the specified limits.

The limits on allowable magnetic noise are especially important for gradiometer measurements, which measure the difference in the magnetic field between two sensors. On GeoSurv II this would be across the wingspan since the magnetometers are located in the wingtips. Gradiometer measurements can typically be on the order of a few nanoTeslas which is quite small compared to the total field value which can range from $20,000 \mathrm{nT}$ to $60,000 \mathrm{nT}$ depending on geographic location. Since gradiometer measurements are so small, it is necessary to eliminate magnetic noise as much as 
possible to increase the precision of the gradiometer measurements. SGL currently operates with a horizontal gradient noise level of less than $0.005 \mathrm{nT} / \mathrm{m}$.

\subsection{Research Objectives}

The primary objective of the magnetic signature control research project is to eliminate the magnetic noise experienced at the wingtip magnetometer locations on GeoSurv II to increase the resolution of the magnetic field survey measurements. Due to the complex nature of magnetic fields. it is not feasible to completely eliminate the magnetic noise at sensor locations as long as there are magnetic components onboard. Therefore, the objective of this research is to reduce the magnetic noise to levels that are within the design specifications. This is accomplished by adhering to the "reduce as much as possible, then compensate" design principle as detailed below:

1. Minimize the use of ferromagnetic material. Position remaining ferromagnetic materials as far from magnetometers as possible.

2. Minimize number of magnetic components. Position remaining magnetic components as far from magnetometers as possible.

3. Minimize the effect of the remaining magnetic components through shielding and passive/active cancellation. Magnetic shielding diverts the magnetic flux from sources so that their magnetic fields are locally contained. Cancellation involves the positioning of additional magnetic sources such that the net effect is less than that of the original magnetic component alone. Active and passive cancellation are used for time-varying and time-invariant sources, respectively.

4. Configure the remaining magnetic field sources such that the net magnetic noise at wingtip magnetometer locations is minimized. 
Often, as is the case of the current GeoSurv II prototype, the replacement of all ferromagnetic materials and magnetic components with nonmagnetic counterparts is not feasible, and thus significant attention must be spent on items 3 and 4, above. Additionally, since the GeoSurv II UAS is much smaller than a typical survey aircraft, magnetic noise sources will be in much closer proximity to the wingtip magnetometers, limiting the ability to implement item 2 effectively. For this reason, the research objectives of this thesis are as follows:

1. Develop a test procedure to identify magnetic components and ferromagnetic material onboard GeoSurv II. This is necessary due to the proximity of magnetic noise sources to wingtip magnetometer locations. Since on GeoSurv II it is often not feasible to increase the source-sensor distance, it is necessary to identify magnetic noise sources so that they can replaced or configured to yield reduced net magnetic noise at wingtip sensor locations.

2. Develop and experimentally validate a modelling strategy for all components expected to contribute to the magnetic interference at wingtip magnetometer locations. These mathematical models can then be used for any theoretical analysis, as well as optimization and cancellation strategies.

3. Develop and experimentally validate, at the UAS-scale, a source configuration optimization strategy designed to minimize wingtip magnetic interference. This strategy configures magnetic noise sources, which cannot be replaced or negated, to yield a reduced net magnetic interference vector at wingtip magnetometer locations.

4. Develop and experimentally validate a passive cancellation strategy such that the magnetic signature of individual sources is reduced. This strategy will be useful for individual magnetic noise sources that cannot be replaced or reconfigured. 


\subsection{Research Contributions}

The research contributions generated from this project include:

1. A novel design optimization strategy was implemented using a genetic algorithm to configure each magnetic noise source to minimize the net effect at regions of interest, such as the wingtip magnetometer locations. It performs a stochastic heuristic search to determine the optimum feasible configuration for magnetic noise sources. The Genetic Algorithm Magnetic Source Optimization (GAMSO) tool was validated experimentally, and is shown to be capable of reducing the net magnetic noise of onboard servos by $98.8 \%$ and $85.8 \%$ at the starboard and port wingtip magnetometers, respectively, relative to the current GeoSurv II prototype configuration.

2. A novel passive cancellation strategy, the Cancellation Magnet Configuration (CMC) strategy, designed to minimize the effect of each source of magnetic noise. The CMC strategy involved the utilization of permanent magnets of opposite polarity in close proximity to the sources which they are intended to minimize. This is intended to locally minimize the magnetic noise of each source to reduce the far-field effect. The CMC strategy was experimentally validated, and was shown to reduce the magnetic signature of a Hitec HSR-5990TG servo by $76.2 \%$ at a distance of $0.52 \mathrm{~m}$.

3. A thorough testing procedure document for experimental analysis of the magnetic signature of unmanned aircraft systems and their components is presented. The testing methods presented may be applied to other systems that must be magnetically quiet. Recommendations for comprehensive testing are given, and form the basis for a metric which describes the overall magnetic signature of the system. Testing was conducted at a magnetically quiet facility to determine 
the magnetic signature of the GeoSurv II prototype and its components.

4. An improved method for characterizing servos as simple magnetic dipole sources. The Dual Permanent Magnetic Dipole (DuPMaD) and Permanent Magnetic Dipole (PMaD) characterization strategies are presented. They are an improvement over a strategy presented by Wells [1], yielding a better fit to data collected along multiple measurement planes. Magnetic components are modelled using one (PMaD) or two (DuPMaD) dipoles that may be placed anywhere within the component geometry, and may have the magnetic dipole vectors in any orientation. Additionally, an improved experimental testing apparatus, environment, and procedure allows for a more accurate representation of magnetic components. The results were verified through a finite element method analysis using COMSOL Multiphysics software.

\subsection{Outline}

There are nine chapters in this thesis. A brief description of Chapters 2 to 9 is provided below.

\section{Chapter 2: Magnetic Field Theory}

The basic physics of magnetic fields required to understand the contents of this thesis are described. Sources of magnetic fields are outlined, the magnetic point dipole is introduced, and a discussion of Earth's geomagnetism is presented. A literature survey of present strategies for dipole modelling, testing, optimization, and cancellation is described. 


\section{Chapter 3: Measuring Magnetic Fields}

Facilities used for testing magnetic fields are described. An explanation of the operation of fluxgate magnetometers and optically pumped Cesium magnetometers is provided. The measurement system to be used on GeoSurv II is also presented.

\section{Chapter 4: Testing and Analysis of Components of GeoSurv II Prototype}

This section presents the procedure, analysis, and results from a novel test strategy used to determine the magnetic signature of individual aircraft components. The magnetic signature of the servo actuators, engine and engine assembly, and flight avionics were experimentally investigated using several different testing procedures. The servo actuators were found to have the largest magnetic signature.

\section{Chapter 5: Characterization Strategies}

Two characterization strategies, DuPMaD and PMaD, utilizing permanent magnetic dipoles are presented. Two servos, the Hitec HSR-5990TG and the Hitec HSM7990TG, are characterized using both characterization strategies and compared to previously developed models. Model performance is verified through the use of a simulation using COMSOL Multiphysics. The DuPMaD and PMaD schemes are shown to produce nearly identical results; therefore, since the PMaD strategy is simpler, it was selected for use in optimization and cancellation strategies.

\section{Chapter 6: Cancellation Strategies}

The development of the novel Cancellation Magnet Configuration (CMC) strategy is presented, along with a description of magnetic cancellation strategies. The CMC strategy aims to minimize the magnetic far-field through the use of a cancellation magnet. The results of the CMC strategy are experimentally validated for the Hitec 
HSR-5990TG, showing that CMC is an effective strategy for decreasing the far-field effect.

\section{Chapter 7: Testing and Analysis of Assembled GeoSurv II Prototype}

This section presents the procedure, analysis, and results from a novel test strategy used to determine the magnetic signature of the GeoSurv II prototype. A series of testing is presented, and a benchmark of the magnetic signature of the UAS is determined. The GeoSurv II prototype was found to have a magnetic signature larger than the allowable magnetic noise, and as such it is necessary to reduce the magnetic noise using magnetic signature control strategies.

\section{Chapter 8: Genetic Algorithm Magnetic Signature Optimization}

The development of the Genetic Algorithm Magnetic Signature Optimization (GAMSO) strategy is presented along with a brief description of the basic components of a simple genetic algorithm. The magnetic field at wingtip magnetometers is modelled using the PMaD characterization of the Hitec HSR-5990TG to represent the 14 servos onboard the current GeoSurv II prototype. The performance of GAMSO is verified using a highly constrained subset of solutions to prove that GAMSO consistently reaches the global minimum. An optimized solution for the configuration of GeoSurv II is determined and experimentally validated.

\section{Chapter 9: Conclusions and Recommendations}

Major conclusions from research involved in this thesis are provided, as well as recommendations for future work. 


\section{Chapter 2}

\section{Magnetic Field Theory}

This chapter will outline the basic theory applicable for attenuation of magnetic interference. A brief explanation of the nature of magnetic fields, along with sources of magnetic interference will be presented.

\subsection{Magnetic Flux Density and Magnetic Field In- tensity}

The term magnetic field may refer to the B-field or to the H-field, which correspond to the magnetic flux density and magnetic field strength, respectively. Magnetic flux density, also known as magnetic induction, is measured in Tesla, T. Magnetic flux lines form continuous loops and thus are said to be solenoidal. The magnetic flux entering a closed surface must be equivalent to the magnetic flux exiting the closed surface. This is shown mathematically using Gauss's Theorem:

$$
\oint_{S} B \cdot d A=0,
$$

for some closed surface $\mathrm{S}$, through area $d A$. The B-field is divergenceless, with no sources or sinks. hence there are no points where the magnetic flux lines begin and 
end. For example. outside of magnets the magnetic flux lines enter near the south pole, and exit near the north pole; within magnets, the magnetic flux lines continue from south to north pole, therefore there are no discrete start or end points for the magnetic flux lines. In this thesis, the term magnetic noise source will refer to a feature that produces magnetic flux lines, and any reference to the magnetic field should be interpreted as referring to the magnetic flux density.

Magnetic field strength, also known as magnetizing force, is measured in $\mathrm{A} / \mathrm{m}$. Unlike the B-field, the H-field is not divergenceless, meaning that it has sources and sinks. Within a magnet, the $\mathrm{H}$-field lines are oriented in the opposite direction to the B-field lines. and the H-field lines appear to originate on the horizontal surfaces of the magnet [4].

In free space, magnetic flux density and magnetic field strength may be used to describe the same property, since they are related through the magnetic permeability of free space, $\mu_{o}$, which has a value of $4 \pi \cdot 10^{-7} T \cdot m / A(H / m)$ :

$$
B=\mu_{o} H
$$

The magnetic permeability is a measure of the ability of a material to conduct magnetic flux. Materials with larger magnetic permeabilities indicate that the material will conduct magnetic flux more readily than a material with a lesser permeability. Most often the permeability of a medium is specified by its relative magnetic permeability, $\mu_{r}$, which is the the ratio of the permeability of the medium being defined to the permeability of free space.

Within magnetic materials, the magnetization, $M$, of the material must be taken into account when relating the $\mathrm{H}$-field and the $\mathrm{B}$-field. The magnetic effect of a 
magnet or magnetic material is often defined using the magnetic moment, which defines the force that a magnetic field will exert on some electric current, expressed in $A \cdot m^{2}$. The magnetization is the mean magnetic moment per unit volume of a medium, measured in $A / m$. In a magnetic medium, the $\mathrm{B}$-field is calculated using the following relationship:

$$
B=\mu_{o}(H+M) .
$$

It is quite difficult to know the exact level of magnetization within a medium due to a variety of factors. Conveniently, air may be assumed to be nonmagnetic, and thus is treated as free space, meaning that it is possible to use equation (2.2) to solve for the magnetic field strength directly provided that the magnetic flux density is known through measurements.

The total magnetic field at any given point is the sum of the vector field contributions of all sources. Therefore, when determining the magnetic field, all sources must be taken into consideration. Often, for convenience, sources with small magnetic field contributions at the measurement point may be neglected.

\subsection{Sources of Magnetic Fields}

Magnetic field sources may be classified as either a moving electric charge or a magnetic moment, which are equivalent to current loops. Electromagnets are classified into the moving electric charge category. Permanent magnets and ferromagnetic materials are classified into the magnetic moment category. Sources of magnetic fields are akin to sources of magnetic interference, and as such are treated as one and the same. 


\subsubsection{Electromagnets}

Magnetic fields are created whenever there is a moving electric charge such as an electric current. The magnitude of the magnetic field produced by an electromagnet is proportional to the current flowing through the circuit [5]. The direction of the magnetic field produced from an electromagnet can be found using the right-hand rule; the natural curl of the fingers will indicate the direction of the magnetic field produced when aligning the thumb of the right-hand with the direction of current flow, as shown in Figure 2.1.

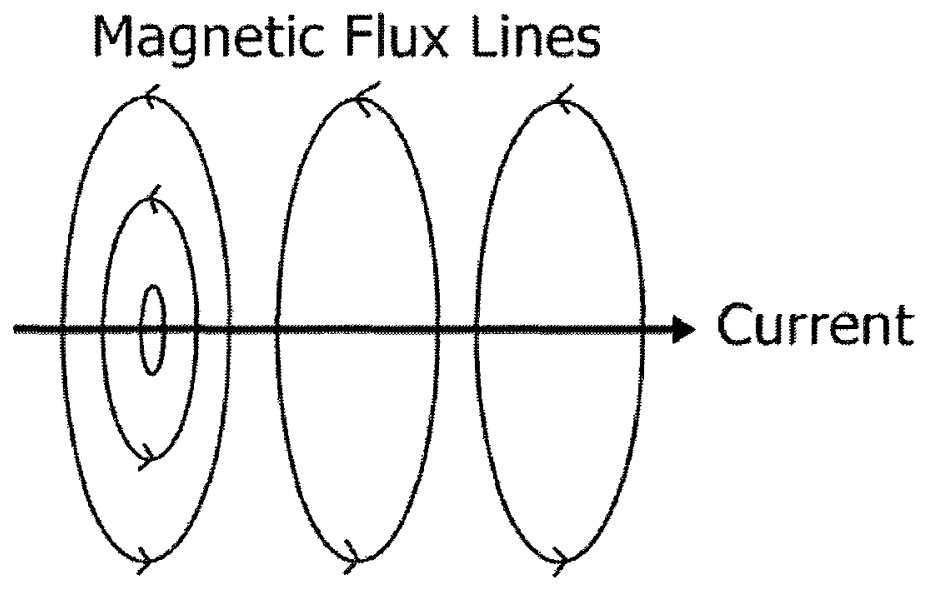

Figure 2.1: Basic electromagnet showing the magnetic flux lines wrapping around the current path, following the right-hand rule.

Electromagnets are the magnetic field source of choice for many applications since they are capable of producing much larger magnetic fields than permanent magnets, and also because the magnetic field is easily controlled by varying the current. A special type of electromagnet called a Bitter magnet, is capable of producing the largest man-made fields, generating brief $45 \mathrm{~T}$ fields [4].

Also belonging to the electromagnet category are eddy currents. Eddy currents are generated whenever there is a changing magnetic field applied to an electrically 
conductive material. They flow in planes perpendicular to the applied magnetic field, in closed loops [6]. Magnetic fields generated by eddy currents are typically small, but can be an issue depending on the size and proximity of the conductive material to some measurement point.

GeoSurv II contains many electromagnetic sources. The most prominent sources are within the fuselage in the flight and mission avionics, where there are current loops which will generate static and transient magnetic fields. Additionally, the GeoSurv II prototype is controlled by servo actuators which utilize electromagnets to produce torque. Furthermore, the wiring runs leading to each servo actuator will be a source of magnetic field onboard GeoSurv II. The magnetic field produced by any wiring can be significantly reduced using the twisted pair method, which involves twisting wiring pairs around one another such the magnetic field produced by one wire will cancel with the other.

Eddy currents are an issue for all geomagnetic survey aircraft, including GeoSurv II. Local magnetic ficld variations result in a transient magnetic field as experienced by the moving survey aircraft. If there are electrically conductive components on the aircraft, then they will generate eddy currents which are largest during angular variations resulting from flight manoeuvres. Design requirement 3.1.3 - 7 [3] was specified to avoid having electrically conductive material near the magnetometers. The magnetic fields produced by eddy currents which are farther than $0.3 \mathrm{~m}$ away from the magnetometers will not be a major source of magnetic noise for the UAS. It is also worth noting that the carbon fibre airframe is electrically conductive to a lesser degree than most metals, but may still present a problem when dealing with magnetic signature of the UAS. For this reason, it may be beneficial to use fibreglass for the construction of the wingtip fairings. 


\subsubsection{Magnetic Materials}

Magnetism in materials is generated by the electrons within. The spin motion of electrons about their own axis and the orbital rotation about the nucleus produce the magnetic moment of an individual electron. The movement and spin of the electrons essentially constitutes a moving electric charge, hence magnetic materials are often thought of as a collection of current loops [7]. In most atoms, the electrons will spin and orbit in such a way that the magnetic moment of each will cancel. In ferromagnetic materials, the magnetic moments of the individual electrons will combine to create a net magnetic moment. The net magnetic field of a material is due to the vector sum of the magnetic moments of all the electrons within the material [5].

Magnetic order describes the degree to which the atomic structure of a material behaves ferromagnetically. It is a relatively low temperature phenomenon, and occurs well below room temperature for most materials. Curie temperatures greater than 500 $\mathrm{K}$ are needed for room temperature applications, which occurs in no more than $20 \%$ of known magnetic materials [4]. At the Curie temperature, the magnetic order of the specimen is destroyed and the specimen behaves paramagnetically. The late $3 \mathrm{~d}$ metals such as iron, cobalt, nickel, and gadolinium, and their alloys, including intermetallic compounds and interstitial alloys, are frequently ferromagnetic [4]. Dilute alloys which contain less than $10 \%$ magnetic atoms cannot be expected to order magnetically at room temperature, if at all.

Magnetic materials respond to changes in applied magnetic field strength by varying their level of magnetization, thus varying the magnetic field produced by the material. For example. if the strength of the applied magnetic field is increased, then the specimen will become more magnetized, and vice versa. This behaviour is more 
commonly known as magnetic hysteresis [7].

Magnetic materials consist of many magnetic domains, which are tiny regions that each have net magnetization in a certain direction. The domains are separated by thin regions known as domain walls where the direction of magnetization rotates from one domain to the next. When a ferromagnetic specimen is initially formed, the magnetic domains will all be oriented randomly such that the net magnetization of the sample will be zero. When a ferromagnetic specimen is introduced to an applied magnetic field, the magnetic domains will attempt to orient themselves parallel to the applied magnetic field. This will occur incrementally until all of the domains are aligned with the applied field, a state that is referred to as saturation magnetization [4].

If the applied field is removed at any point during the magnetization process, the specimen will retain some of its magnetization, known as the remanent magnetization [4]. Permanent magnets are a great example of this process. The material used for the permanent magnet is exposed to an applied magnetic field, usually to saturation, such that when the magnetic field is removed, the specimen will have a remanent magnetization, and hence will behave as a permanent magnet. A typical hysteresis curve with diagrams of domain orientations is shown in Figure 2.2.

Additionally, ferromagnetic specimens may become magnetized through cold working. This is because the strain, particularly plastic strain, induced during cold working can cause the magnetic domains to be reoriented, hence changing the net magnetization of the specimen [9]. Other factors affecting the magnetization of a ferromagnetic specimen include thermal gradients, vibration, and the rate of change of the applied magnetic field. Therefore, since it is difficult to know the exact magnetic history of a specimen, it is extremely difficult to know precisely the magnetization of a specimen through time. 


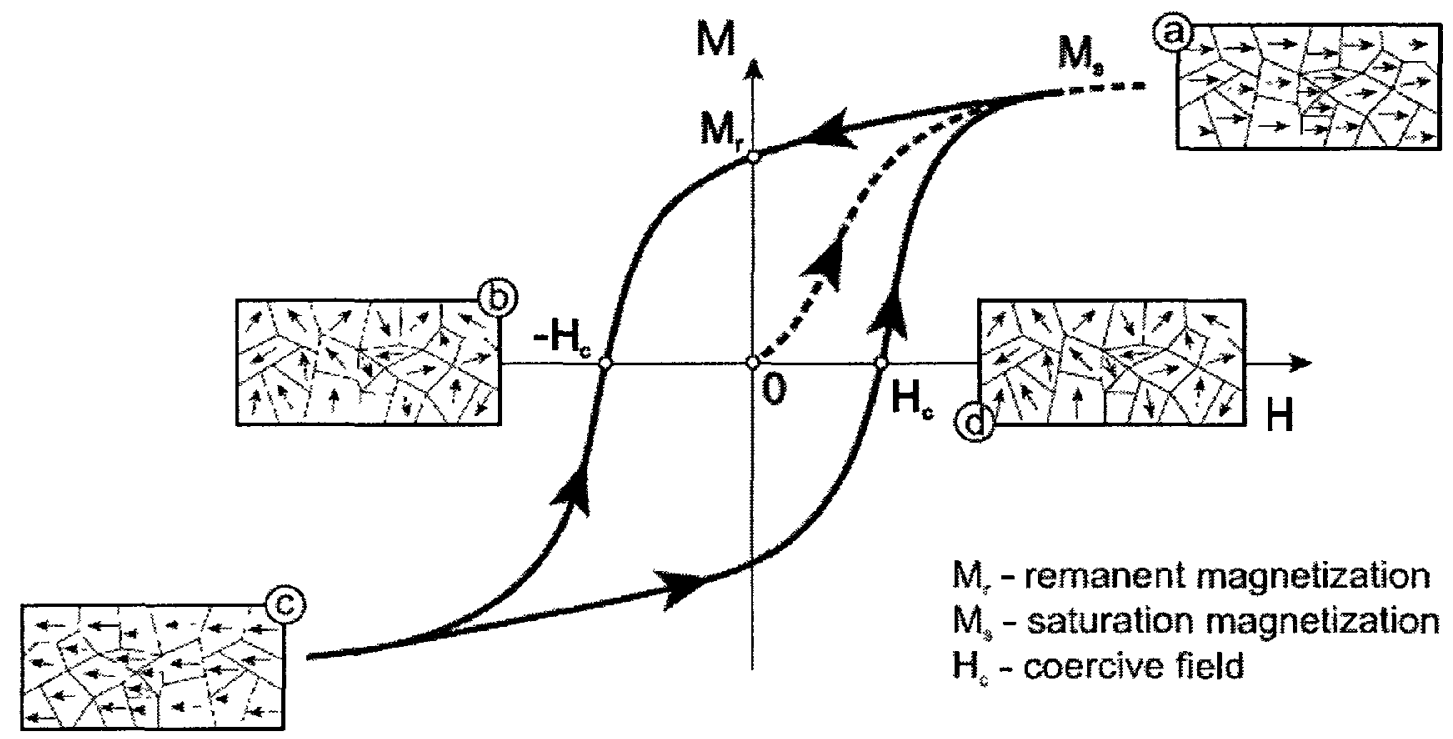

Figure 2.2: Basic hysteresis loop for a magnetic material [8]. The material will become magnctized with an applied magnetic field. If the applied field is large enough, the magnetization will reach saturation. If the applied field is removed, the material will retain some magnetization, known as the remanent magnetization. If the applied field is reversed, the magnetization will also reverse.

It is possible to decrease the magnetization of a specimen in one of two ways. The first is through a process called degaussing. This involves cycling the specimen through a reversing applied magnetic field that slowly diminishes with time such that the magnetization of the object is decreased towards zero. It is impossible to fully demagnetize the specimen using this technique. The other method for demagnetizing a specimen is to anneal the specimen above its Curie temperature, which is specific to each material. By annealing the specimen above the Curie temperature, the magnetic domains within the specimen will randomly orient upon cooling in a magnetic fieldfree environment, yielding a specimen with net zero magnetization [4].

The main source of magnetic noise onboard GeoSurv II comes from the permanent magnets within the servo actuators. These are magnetic materials that produce large magnetic fields. To a lesser extent, ferromagnetic materials used for fasteners 
and other components will increase the magnetic signature of the UAS. Although the engine block is composed of aluminum which is nonmagnetic, components in the engine, such as the crankshaft and connecting rods, will be responsible for some magnetic noise. To reduce the combined effect of magnetic materials, the best strategy is to replace the magnetic components with nonmagnetic equivalents to the largest possible extent.

\subsection{The Magnetic Dipole}

Magnetic field sources are made up of one or more dipoles, as well as possible multipole components. Magnetic dipoles are defined by their magnetic dipole moment, $\vec{m}$, which specifies the force that the magnetic dipole will exert on some electric current. A magnetic dipole is an elementary magnetic quantity which may be represented by a tiny current loop of any shape. as long as the current flows in a plane [4]. The magnetic dipole moment of a current loop is defined as follows:

$$
m=I \cdot A
$$

where $I$ is the current circulating within the loop in Amperes, and $A$ is the area of the loop in square metres, thus the units of $\vec{m}$ are $A \cdot m^{2}$. The magnetic dipole moment is oriented normal to the plane of current flow, in a direction that is determined by the direction of current flow using the right-hand rule.

The magnetic field due to a magnetic dipole may be calculated using the following, which is an expansion of Gauss's Law for magnetism:

$$
\vec{B}=\frac{\mu_{o}}{4 \pi}\left[\frac{3(\vec{m} \cdot \hat{r}) \hat{r}-\vec{m}}{\|\vec{r}\|^{3}}\right]
$$


where $\hat{r}$ is the unit vector of the position vector, $\vec{r}$, which extends from source to measurement point. It is important to note from (2.5), that the dipole field will decay proportionally to the inverse cube of distance. Magnetic fields decay at a rate proportional to $1 /\|r\|^{n}$ where $n=3$ for dipole sources and $n \geq 4$ for multipole sources $[10]$. Hence, in the far-field, which is defined by regions at distances greater than three times the source's largest dimension, the dipole field will dominate while the multipole field will contribute to a significantly lower portion of the total magnetic field. Figure 2.3 shows the difference between a quadrupole and dipole field.

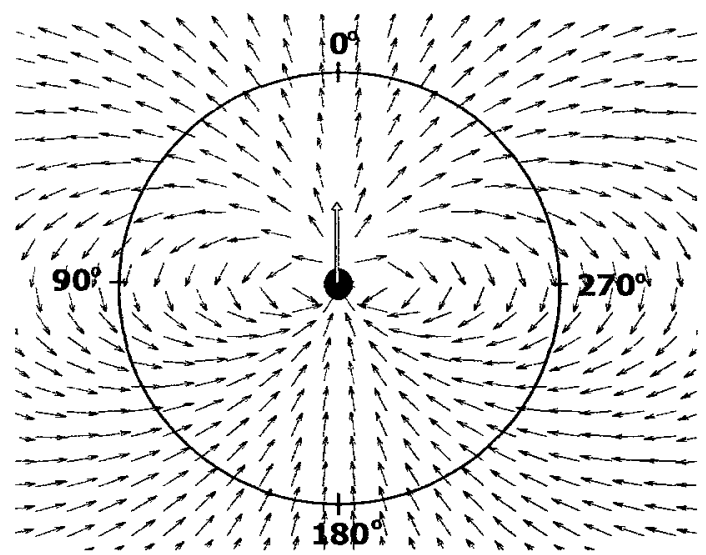

(a) Dipole Field

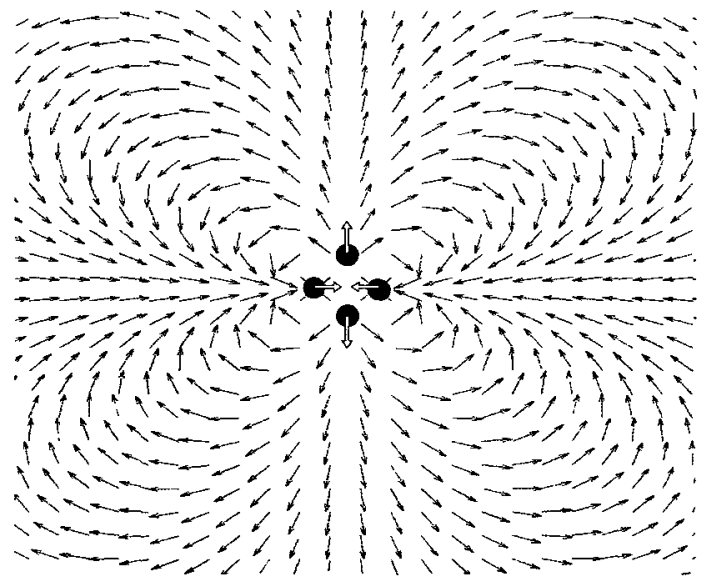

(b) Quadrupole Field

Figure 2.3: Comparison of dipole and quadrupole flux lines. Magnetic quadrupoles may be represented by four magnetic dipoles in close proximity with opposing orientations. The orientation of each dipole is indicated.

Additionally. if rotating the dipole source about some fixed point, and taking measurements at some fixed point in the measurement plane, it can be noted magnetic total field will vary from $\left\|\vec{B}_{\max }\right\|$ to $0.5\left\|\vec{B}_{\max }\right\|$. The locations of $\left\|\vec{B}_{\max }\right\|$ will correspond to the points at which the dipole moment vector is most closely aligned with the position vector. This result is illustrated in Figure 2.4 for the magnetic point dipole shown in Figure 2.3a. 


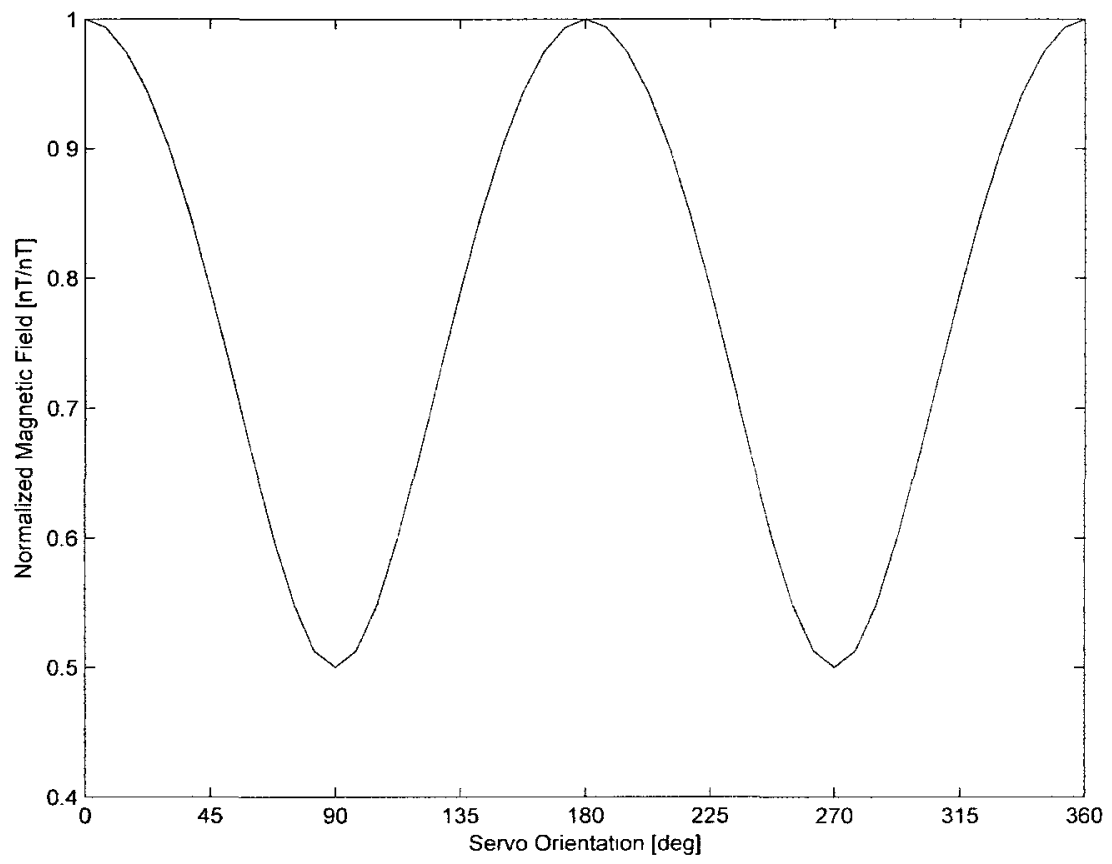

Figure 2.4: Behaviour of total magnetic field for dipole rotating about some fixed point. The field varies between $\left\|\vec{B}_{\max }\right\|$ and $0.5\left\|\vec{B}_{\max }\right\|$ for when the magnetic moment vector is aligned with and normal to the position vector, respectively.

(2.5) is useful since most magnetic field sources may be accurately modelled as a magnetic dipole when performing a far-field analysis. If the distance from field source to measurement point is greater than three times the largest dimension of the field source, then the magnetic dipole approximation of magnetostatics may be utilized. The assumption made with the magnetic dipole approximation is that one or more magnetic noise sources may be modelled as a single point dipole provided that the magnitude of the position vector from the centre of the source geometry, $\vec{r}$, is sufficiently large $[1,11]$. For multiple sources, the source geometry is defined by the longest distance between any two sources [12]. This approach is alternatively known as far-field analysis. 


\subsection{Geomagnetism}

The magnetic field at any given location on Earth is due primarily to three contributors: Earth's main field, diurnal effects, and magnetic anomalies. Magnetic fields measured during airborne geomagnetic surveys can range from 20,000 to $70,000 \mathrm{n}$ T which is a result of all three contributors. Magnetic fields strengths provided in this section are at the Earth's surface or near-surface unless otherwise specified.

\subsubsection{Earth's Main Field}

Earth's main field behaves as a large magnetic dipole. Currents flowing in the liquid outer core of Earth are responsible for the main field, which obtains its principal alignment from the axial spin of the Earth [13]. Magnetic flux lines originating in the outer liquid core travel around the Earth from the Magnetic South Pole to the Magnetic North Pole, which is opposite to the convention used to describe the poles of magnetic dipoles [14]. The location of each pole changes gradually over time due to slow changes in the flow processes within the liquid core. Additionally, the magnetic field of the Earth has reversed several times throughout Earth's history in a process that is not well understood. Earth's main field is strongest near the poles, and weakest near the equator, with values of approximately $60,000 \mathrm{nT}$ and 30,000 $\mathrm{nT}$, respectively [13].

\subsubsection{Diurnal Effects}

Variations in the magnetic field that occur on short time scales are often due to diurnal variations. Diurnal variations are due to electrical currents in the Earth's ionosphere. The solar wind, composed of neutral hydrogen atoms, protons, and electrons, originates from the sun and interacts with Earth's main field to cause variations on the order of roughly $5 \mathrm{nT}$. Additionally, atoms within the ionosphere are ionized 
by ultraviolet light from the sun, causing strong electric currents which can generate magnetic fields of up to $80 \mathrm{nT}$ : these variations subside at night when the local ionosphere is facing away from the sun [14]. Furthermore, solar storms may expel increased quantities of charged particles causing sudden increases in the geomagnetic field intensity. Other variations that may occur on short time scales may be due to the rotation and/or orbital motion of the Earth, sun and moon [15].

\subsubsection{Magnetic Anomalies}

Magnetic anomalies on shorter scales (less than $500 \mathrm{~km}$ ), are generally correlated with geological surface features [14]. These are often due to the magnetization of crustal rocks at depths ranging from a few meters to hundreds of kilometers [15]. Local magnetic anomalies provide information about the properties of the crust's geological structures [15]. The magnetic fields generated by concentrations of magnetic ores are known to produce local field variations of up to $10,000 \mathrm{nT}$ at a distance of $25 \mathrm{~m}$ [14]. The magnetic field variations generated by anomalies decrease rapidly with altitude which results in minimal contributions at space elevations [13]. For this reason, geological anomalies are more easily identified at lower altitudes, hence providing motivation to develop unmanned geomagnetic survey aircraft.

\subsection{Literature Survey}

The section covers a literature survey that was conducted for the topics explored in this thesis: dipole modelling strategies, testing strategies, optimization strategies, and cancellation strategies. 


\subsubsection{Dipole Modelling Strategies}

Distributed multipole models, which model sources as a group of magnetic point dipoles, have been proposed by Lee and Son [16]. This approach was first described to account for varying permanent magnet shapes since single dipole models are only accurate for needle-like magnets. Distributed multipole models account for different magnet shapes by distributing a finite number of magnetic dipoles throughout the geometry of the magnet. Olsen and Lyon [10] use a similar approach, but take into consideration spherical and cylindrical multipole sources. Also, Roy [17] presents a method for characterizing the magnetic field of a spacecraft in terms of its multipole coefficients. The multipole approach is beneficial because it allows for the near-field to be modelled with increased accuracy over single dipole models which is useful for real-time modelling of magnetic fields within motors. However, increasing the number of dipoles adds complexity which may not be necessary when studying the magnetic far-field, as is the case for the GeoSurv II application.

A simple and accurate method used to characterize a single point source was outlined by Zaffanella, Sullivan, and Visinteiner [12]. The method involves treating sources as a single point dipole provided that the measurement points are in the far-field. The determination of the magnetic dipole components $\left(\vec{m}_{x}, \vec{m}_{y}, \vec{m}_{z}\right)$ is done through fitting (2.5) to magnetic field components measured experimentally at three points. The assumption is made that the position vector acts from the centre of the source volume to the measurement point, which may not necessarily be true. Determination of the point dipole location within the source volume is then found through iteration using the magnetic dipole components calculated with the estimated position vector. 
Although the method for characterizing sources presented by Zaffanella et al. is sound, there was not enough experimental data collected to precisely characterize the magnetic moment of a source. Additional measurement points at various orientations and distances are needed to effectively characterize a source. Furthermore, the approach described in Zaffanella et al. involves treating the magnetic point dipole components separately from the position vector, when in fact the six parameters are interdependent and should therefore be calculated at the same time.

The approach outlined by Zaffanella et al. was further expanded by Wells [1] by the addition of an extra magnetic dipole to represent each source in greater detail. The Modified Dipole Ellipse Model (MoDEM) characterized magnetic sources using two point magnetic dipoles of any orientation, with the minor dipole offset in the z-direction of the source from the major dipole. Both dipoles were constrained to be within the geometry of the source. The approach used by Wells utilized a minimization algorithm (MATLAB fmincon) to fit the 10 MoDEM parameters $\left(\vec{m}_{1, x}\right.$, $\left.\vec{m}_{1, y}, \vec{m}_{1, z}, \vec{m}_{2, x}, \vec{m}_{2 . y}, \vec{m}_{2, z}, x_{1}, y_{1}, z_{1}, z_{\text {offset }}\right)$ to data collected experimentally. The experimental data collected involved rotation of the source about its geometric centre at a known distance in a single plane. The experimental method and characterization strategy developed by Wells provides a better representation of the equivalent magnetic dipole than those in Zaffanella et al..

However, the results presented by Wells are biased towards magnetic fields within the plane of testing. All experimental data used for parametrization involved rotation about the source's z-axis in a single plane, resulting in a poor fit between the theoretical and experimental results for the z-component of the magnetic field, as can be seen in Figure 2.5. 


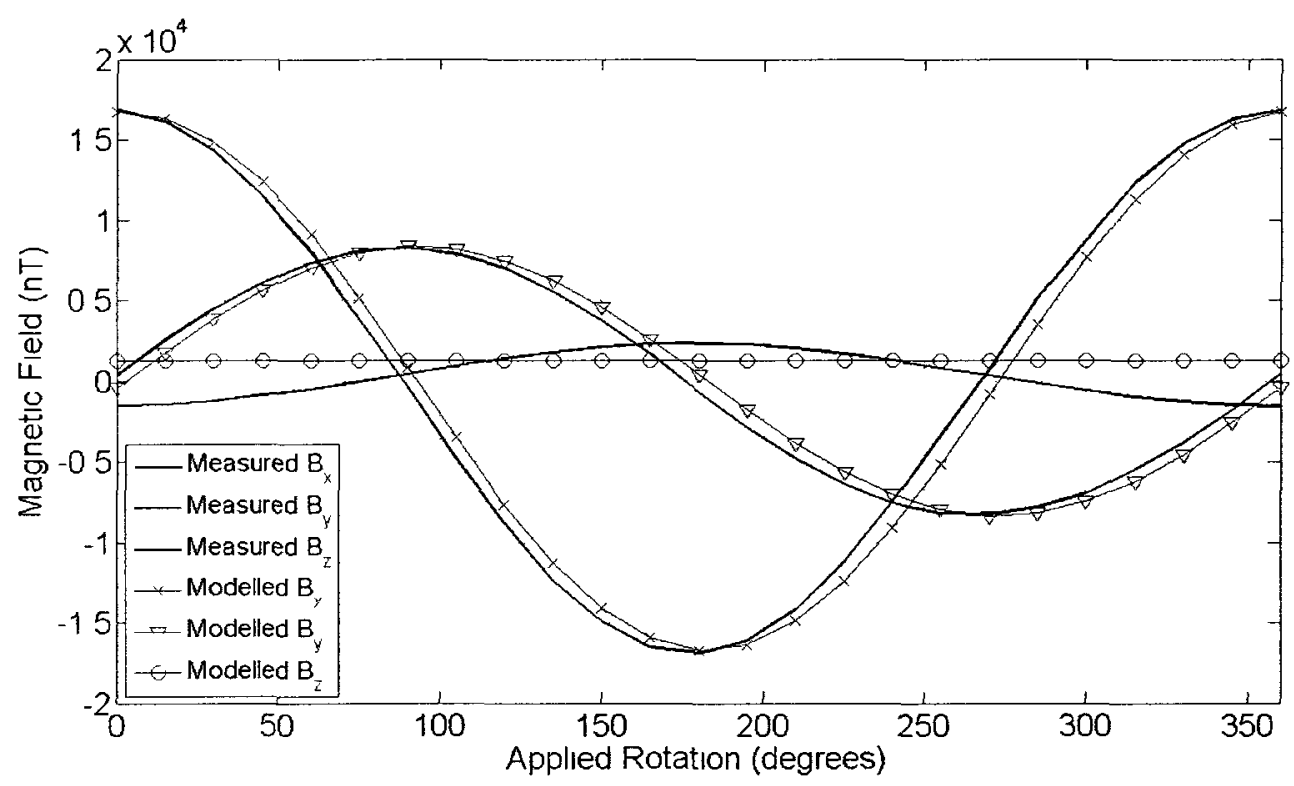

Figure 2.5: Dipole source modelled using Wells' MoDEM model [1]. Note the modelled $\vec{B}_{z}$ component remains at the same value for the entire rotation, and does not follow the measured trend.

This was due to an incomplete characterization of the magnetic dipole component parallel to the axis of rotation. In order to properly characterize all three vector components of the equivalent magnetic dipole it is necessary to perform measurements in multiple planes. The Dual Permanent Magnetic Dipole (DuPMaD) characterization strategy presented in this thesis is a variation of Wells' MoDEM which allows for full degrees of freedom in the placement of the two magnetic point dipoles within the source geometry. and utilizes experimental data collected in multiple planes of rotation at varying distances to provide a more accurate representation of all the vector components of the equivalent magnetic point dipole.

Depending on the size of the source being modelled, it may be necessary to include additional magnetic dipoles to model larger sources of magnetic noise. Mehlem [18] outlines the use of up to 29 independent dipoles to model the magnetic signature of a satellite. This approach is useful for generating rough models of aircraft subassemblies 
without needing to individually test each subassembly. The drawback is that using a large number of dipoles will inherently make the near-field more complex, and will make characterization computationally and experimentally expensive.

\subsubsection{Testing Strategies}

The majority of the current published literature studying magnetic signature reduction strategies for vehicles has been completed for naval vessels. The reason being that naval mines often use the disturbance in the background field caused by the presence of a ship or submarine as a triggering mechanism. Additionally, aircraft similar to those used for geomagnetic surveys may be used to locate submerged submarines through the detection of local magnetic anomalies.

Kildishev, Nyhenuis, Boyko, Dobrodeyev, and Volokhov [19] present a method for determining the magnetic centre of an unmanned underwater vehicle(UUV). The magnetic centre is defined as the location at which the placement of an appropriate compensating dipole and quadrupole will essentially eliminate the first eight coefficients of a spherical harmonic expansion of the magnetic scalar potential. Kildishev et al. [19] locate the magnetic centre by moving a UUV through a series of sense coils. Measurements were taken at incremental distances, allowing for the determination of the optimal size and location of a cancellation dipole near the magnetic centre. The coils used in [19] require a dedicated room within a magnetically quiet facility, which incurs large resources and costs. A simpler and less expensive alternative must be devised for smaller projects such as the GeoSurv II.

An experimental investigation on the magnetic signature of an autonomous rotating wing UAS-magnetometer system was presented by Versteeg, McKay, Anderson, Johnson, Selfridge, and Bennett [20]. The effect of orientation on magnetic signature 
for various separation distances (stinger lengths) between the sensor and UAS was studied. The experimental apparatus used was itself not magnetically quiet. requiring data correction to determine true ralues of the magnetic field at each orientation Additionally, the orientation was correlated to the time elapsed for rotation at a presumed constant rate. but the rate likely was not constant since the platform was rotated manually, leading to errors in the orientation calculation. A better solution is to take readings incrementally at known orientations to maintain explıcit knowledge of UAS orientation. Versteeg et al. [20] also conducted a pullover test, which involved moving the UAS past a fixed sensor to determine the magnetic signature of the UAS. The effect of the powered engine was also studied during the pullover test. However, the data was not correlated to the source-sensor distances. and hence no analysis was completed to pinpoint the locations and magnitudes of the main contributors of magnetic noise. Figures 2.6a and 2.6b show the test apparatus used by Versteeg et al. [20].

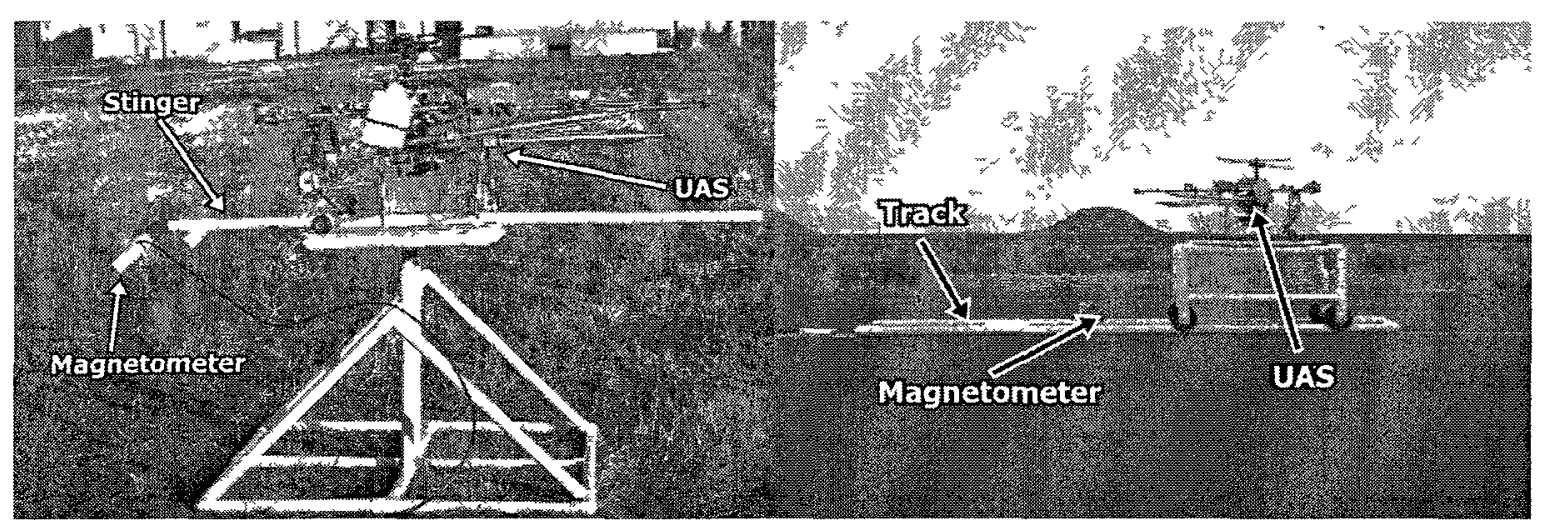

(a) Orientation Test

(b) Pullover Test

Figure 2.6: Experimental apparatus used by Versteeg et al. [20]. In (a), the test rig was rotated manually at a semi-constant rate, allowing for an approximate calculation of heading. In (b), the test rig was translated manually, but data was not correlated to UAS position relative to the magnetometer. 
Using experimental methods that involve moving the vehicle past some fixed sensor, such as was done by Kildishev et al. [19] and in the pullover test by Versteeg et al. [20], it is possible to use different analysis techniques to isolate magnetic dipoles within the vehicle. Ginzburg, Frumkis, Kaplan, Sheinker, Lerner, and Salomonski [21,22] suggest using genetic algorithms and simulated annealing. As well, Alimi, Geron, Weiss, and Ram-Cohen [23] utilized a Levenberg Marquardt algorithm to locate and characterize magnetic dipoles within larger assemblies. These approaches are beneficial for low signal to noise ratios (SNR), which is often the case for magnetic anomaly detection involving relatively large source-sensor distances. For the GeoSurv II testing covered in Chapters 4 and 7, the source-sensor distances are controlled to provide a favourable SNR, allowing simpler analysis techniques to be implemented.

Bono, Overway, Wynn, Allen, Purpura, and Matthews [24-26] present an alternative experimental method which involves attaching a UUV to a three-axis motion table rocking at known frequency. Several total-field magnetometers are positioned around the motion table and on the UUV, and the data was analyzed in the frequency spectrum to localize magnetic field sources. Allen et al. $[25,26]$ also present a method similar to the pullover test discussed by Versteeg et al. [20], which involves a set of magnetometers arranged in a gradiometer configuration located as closely as possible to the movement track. Both approaches were utilized on multiple UUV's, allowing for the localization of several noise sources and the improved selection of suitable magnetometer locations. A similar method to the gradiometer test outlined in $[25,26]$ was used to analyze the magnetic signature of GeoSurv II, as is described in Chapter 7. 


\subsubsection{Optimization Strategies}

Since the magnetic field at any point in space is the vector sum of all magnetic fields at that point, it presents the opportunity to configure the local magnetic field sources so that the net effect in a region of interest is minimized. Previous work was completed on this topic by Wells [1]. Wells' Optimal Field Attenuation (OFA) strategy optimizes the configuration (location and orientation) of a finite number of magnetic field sources such that the net effect in some defined region (or regions) of interest is minimized. The OFA approach yielded promising results which were experimentally validated in [1].

The OFA optimization and experimental validation allowed only rotations about the vertical axis (servo z-axis as defined in [1]). Additionally, in the OFA scheme each source may assume any orientation about the axis of rotation, presenting the possibility of positioning servo actuators in orientations where their mechanical leverage cannot be effectively used. This is disadvantageous from a practical standpoint. Onboard GeoSurv II, the servos must assume orientations which are normal to the coordinate planes of the aircraft to promote ease of installation and effective use of their mechanical leverage. Furthermore, the OFA strategy optimizes over a defined optimization area (2-Dimensions), when in reality it is necessary to optimize over an optimization volume (3-Dimensions), as is the case with GeoSurv II. For these reasons, a new optimization algorithm was developed in this thesis and is presented in Chapter 8.

\subsubsection{Cancellation Strategies}

There are multiple ways to decrease the effect of single magnetic noise sources. Shielding is the most common method of protecting against unwanted magnetic fields. Regions can be shielded using a variety of means depending on the nature of the field 
that is to be negated. Static fields, that is magnetic fields which do not vary with time, may be compensated by super-positioning additional magnetic field sources (permanent magnets or electromagnets) of equal magnitude and opposite polarity such that the magnetic flux lines are locally contained. This method was implemented and experimentally validated as shown in Chapter 6. Transient fields, which are magnetic fields that vary with time, may be compensated by using a pair of Helmholtz coils to create a uniform magnetic field within the region of interest, and an external reference sensor to monitor changes to the background field. The use of three orthogonal Helmholtz coils allows for control of the three vector components of the magnetic field within the region of interest. Shielding of high frequency magnetic fields can be accomplished using a Faraday cage.

Both static and transient magnetic fields may also be compensated for using high magnetic permeability materials, such as $\mathrm{Mu}$-metal, which provide a low reluctance path for the magnetic field. By enclosing regions of interest, in high magnetic permeability materials, it is possible to create regions where the external magnetic field is practically negated within the enclosed region. This will also cause the external magnetic field to be larger near high magnetic permeability materials. An experimental investigation into the use of Mu-metal to shield a servo actuator was conducted by Forrester [27]. The conclusions of [27] indicate that the use of magnetic shielding results in a magnetic field that does not vary significantly with orientation, leading to the conclusion that the effect measured during experimentation was due to the interaction of the high permeability shield with the local background field. This implies that the magnetic flux originating in the servo was effectively contained by the shielding layers. The shielding decreased the mean absolute magnetic field for most testing sequences, which may be seen in Figure 2.7. The use of high magnetic permeability materials for the GeoSurv II application may be useful for large, single 
magnetic noise sources, however the effect on the background magnetic field should be experimentally validated for each source and shielding configuration.
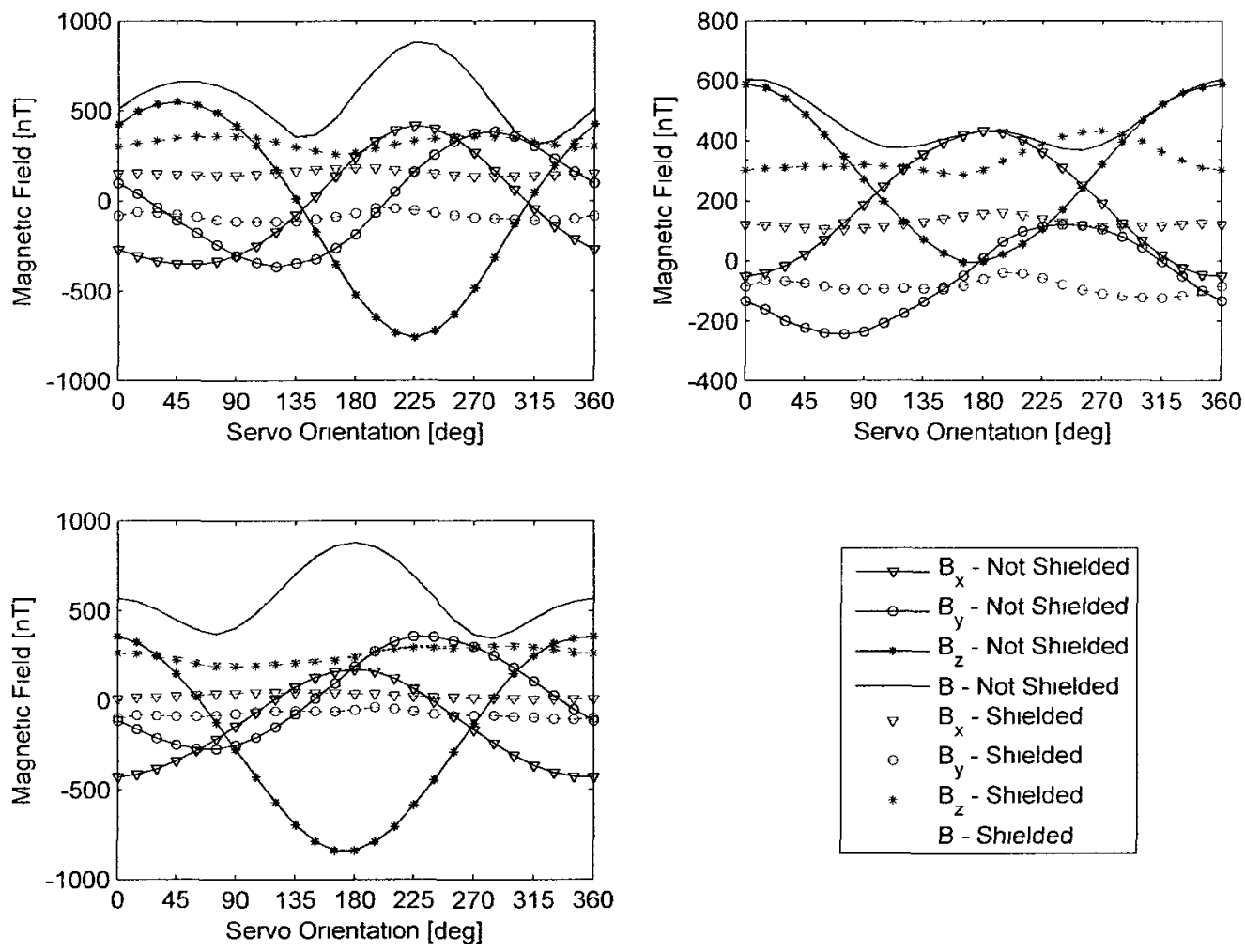

$$
\begin{aligned}
& \longrightarrow-B_{x}-\text { Not Shielded } \\
& \longrightarrow B_{y} \text { - Not Shielded } \\
& B_{z} \text { - Not Shielded } \\
& \text { B - Not Shielded } \\
& \checkmark \mathrm{B}_{x} \text { - Shielded } \\
& \text { - } B_{y} \text { - Shielded } \\
& \text { * } \quad B_{z} \text { - Shielded } \\
& \text { B-Shielded }
\end{aligned}
$$

Figure 2.7: Effect of Mu-metal shield on Hitec HSM-7990TG servo at a distance of $0.33 \mathrm{~m}$ for rotations about the servo's $\mathrm{x}$ - (top left), $\mathrm{y}$ - (top right), and $\mathrm{z}$ (bottom left) axis. The shielded scenario yielded lower mean absolute magnetic field interference over each $360^{\circ}$ rotation. 


\section{Chapter 3}

\section{Measuring Magnetic Fields}

\subsection{Introduction}

There are many different ways to measure the magnetic field, and thus many different types of magnetometers. The choice of magnetometer depends on, among other factors, the range, resolution, physical dimensions, and whether the vector or total field is required for the desired application. Additionally, to measure magnetic fields accurately, measurements must be completed in environments that do not affect the local magnetic field. This chapter will cover the magnetometers and facilities used for testing, as well as the measurement system on the final GeoSurr II design.

\subsection{Facilities for Magnetism Testing}

Facilities for magnetism testing must not have a local magnetic ficld that varies significantly with time or space. Features within the facility such as ferromagnetic materials, permanent magnets, or electromagnets cause magnetic field gradients which can cause measurement errors [15]. The dominant component of any magnetic field is the dipole which decreases proportionally to the inverse cube of distance. Hence, the best way to avoid interfering magnetic fields is to position magnetic objects as 
far away as possible from the measurement location. Refer to Table 3.1 for a list of distances at which some common objects will produce a magnetic field deviation of $1 \mathrm{nT}$.

Table 3.1: List of distances at which some common objects will generate a magnetic field of $1 \mathrm{nT}$. if configured appropriately [15].

\begin{tabular}{|c|c|}
\hline Object & Source - Sensor Distance $[\mathrm{m}]$ \\
\hline \hline Belt buckle & 1 \\
\hline Watch & 1 \\
\hline Screwdriver & 1 \\
\hline Hammer & 4 \\
\hline Motorcycle & 20 \\
\hline Car & 40 \\
\hline Bus & 80 \\
\hline
\end{tabular}

In order to eliminate measurement errors, it is necessary to perform magnetism testing at facilities that have been designed to minimize spatial magnetic field gradients and to reduce the transient field effects towards a constant magnetic field. These are created using primarily nonmagnetic materials, and by positioning any sources of magnetic interference far enough away from the test area to not affect measurements. Some facilities, such as magnetic resonance imaging (MRI) facilities, use shielding to eliminate local magnetic interference when the fields to be measured are extremely small ( $\leq 0.2 \mathrm{nT}$ ) and when equipment is sensitive to large magnetic fields [28]; this is not the case for the testing involved in this project.

Selection of proper testing facilities involves the investigation of all features - buildings, equipment, infrastructure - around the facility to determine if it is suitable for testing. If the local magnetic field gradients are small, changes in the background 
field may be recorded using a reference sensor which is positioned far enough away from the measurement area such that it measures only variations in the naturally occurring background field, allowing transient local field variations to be removed in post-processing. It is important to note that if using vector magnetometers, it is necessary that the reference and measurement sensors be aligned or to have knowledge of their orientations relative to one another.

Testing completed during this project was performed at three primary locations: Carleton University Campus, Natural Resources Canada's Geomagnetic Laboratory, and at SGL's headquarters in Ottawa. These facilities will be described in this section. and their suitability as magnetic field testing environments will be discussed.

\section{Carleton University Campus and SGL Headquarters}

Testing at both the Carleton University Campus and SGL Headquarters was conducted for two main reasons: a) to refine experimental apparatus and testing procedures, and b) to collect measurements in order to determine the approximate magnetic signature of particular components. Both facilities were not ideal for magnetism measurements, but nonetheless useful data was collected. Before any experiments were conducted it was necessary to determine the suitability of each location for testing. Since neither location was built to be magnetically quiet, they were both susceptible to the many sources of magnetic noise within the immediate vicinity.

Therefore, due to the large quantity of magnetic noise sources, the magnetic field gradients within each location are too large to effectively utilize reference sensors. This is because the background variation at the measurement sensor may not be of the same magnitude as at the reference sensor, thus making it difficult to properly remove background transient fields. Therefore, it was necessary to determine the 
magnetic behaviour of each location over the course of one or more days to quantify the expected magnetic noise deviation.

Testing at Carleton University was originally planned in the Visualization and Simulation (VSIM) Building at the South West corner of campus. It was found that there were large fluctuations in the magnetic field associated with elevator use. As can be seen in Figure 3.1, fluctuations of $\approx 2,500 \mathrm{nT}$ are associated with elevator use, potentially followed by an offset in the background field depending on which floor the elevator stopped. 


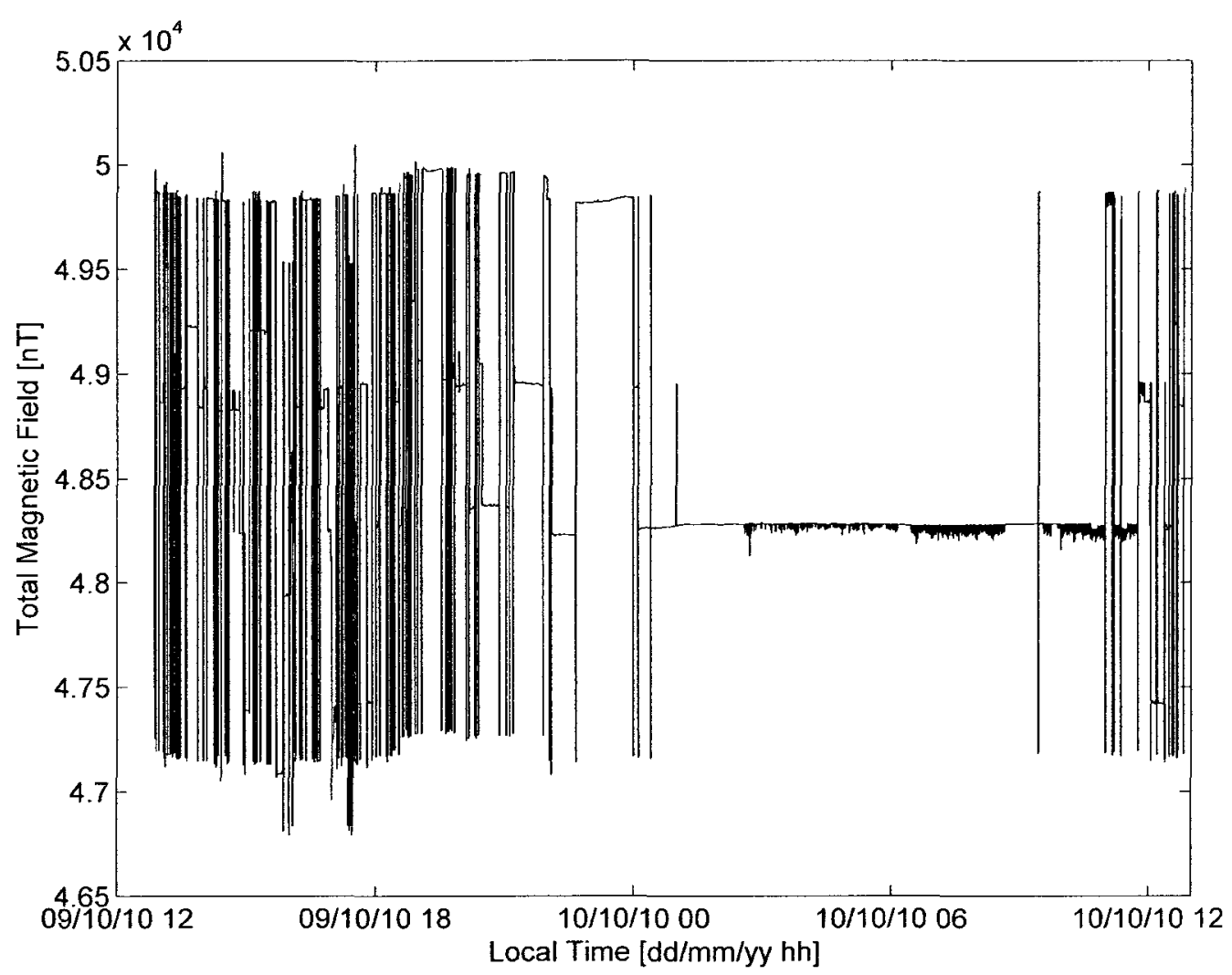

Figure 3.1: Magnetic field behaviour in the Carleton University Visualization and Simulation Building, room 3219 over the course of a day. Measurements were taken from 09 May 2010 at 12:51 EST to 10 May 2010 at 12:51 EST. Movement of the elevator results in $\mathrm{a} \approx 2,500 \mathrm{nT}$ variation, which accounts for the daily fluctuation.

Due to the unsuitability of the VSIM building, two locations for testing in the Mackenzie Engineering (ME) Building were surveyed. Figure 3.2 shows that the magnetic field behaviour in ME2180 over a 24-hour period is relatively undisturbed compared to VS3219. Although ME2180 is still susceptible to large fluctuations in the background field, it was deemed to be the most appropriate location for magnetism testing out of the limited number of locations tested on the Carleton University campus. 


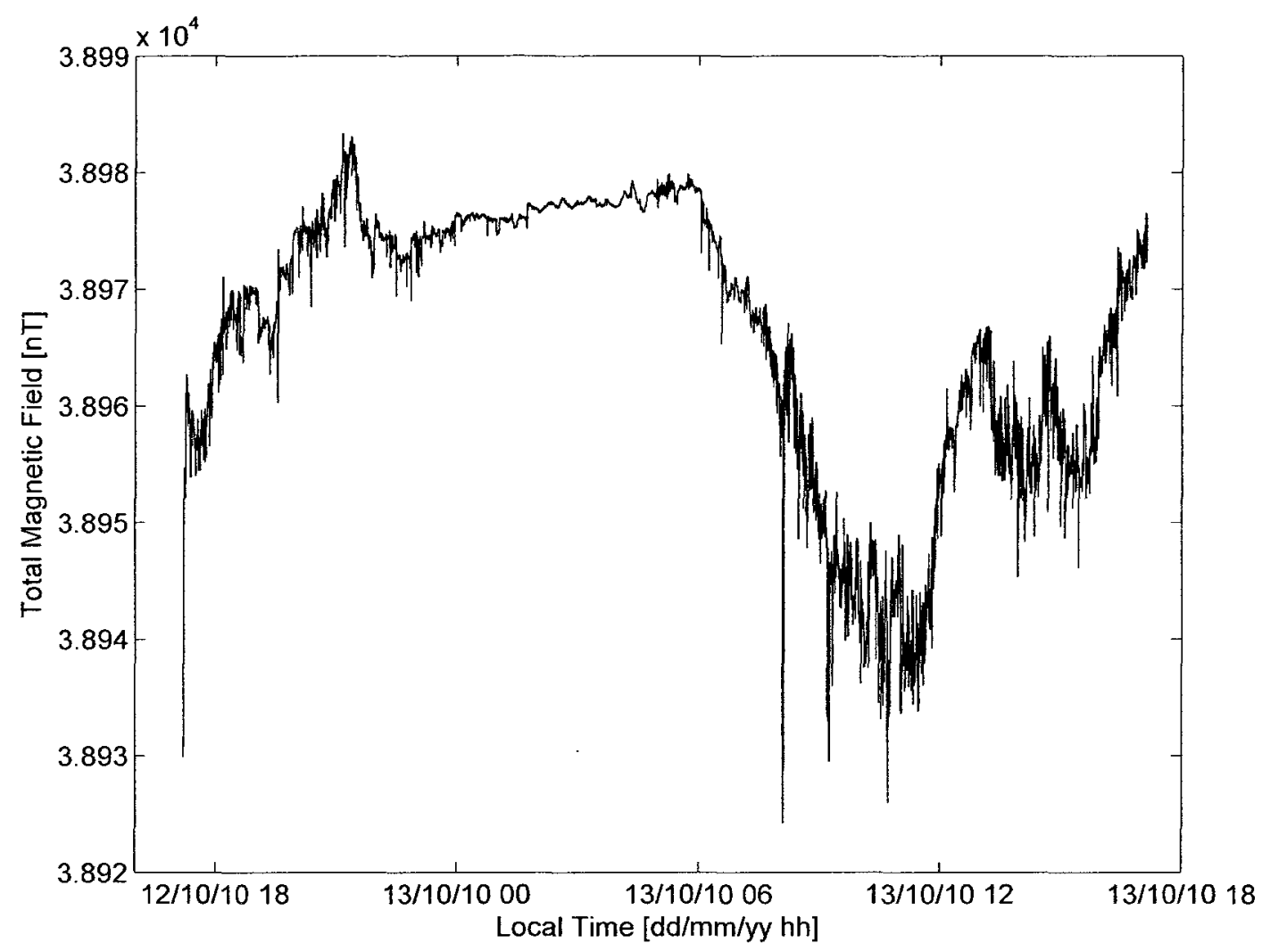

Figure 3.2: Magnetic field behaviour in the Carleton University MacKenzie Engineering Building, room 2180 over the course of a day. Measurements were taken from 12 May 2010 at 17:07 EST to 13 May 2010 at 17:07 EST. The daily variation is $\approx 55 \mathrm{nT}$, with the largest fluctuation being $\approx 35 \mathrm{nT}$.

Additionally, SGL Headquarters was used for shorter testing sequences, lasting less than a few days, and not requiring a completely magnetically quiet environment. As shown in Figure 3.3, the magnetic field fluctuates least between 17:00 and 06:00, thus testing completed at SGL was conducted within this window. 


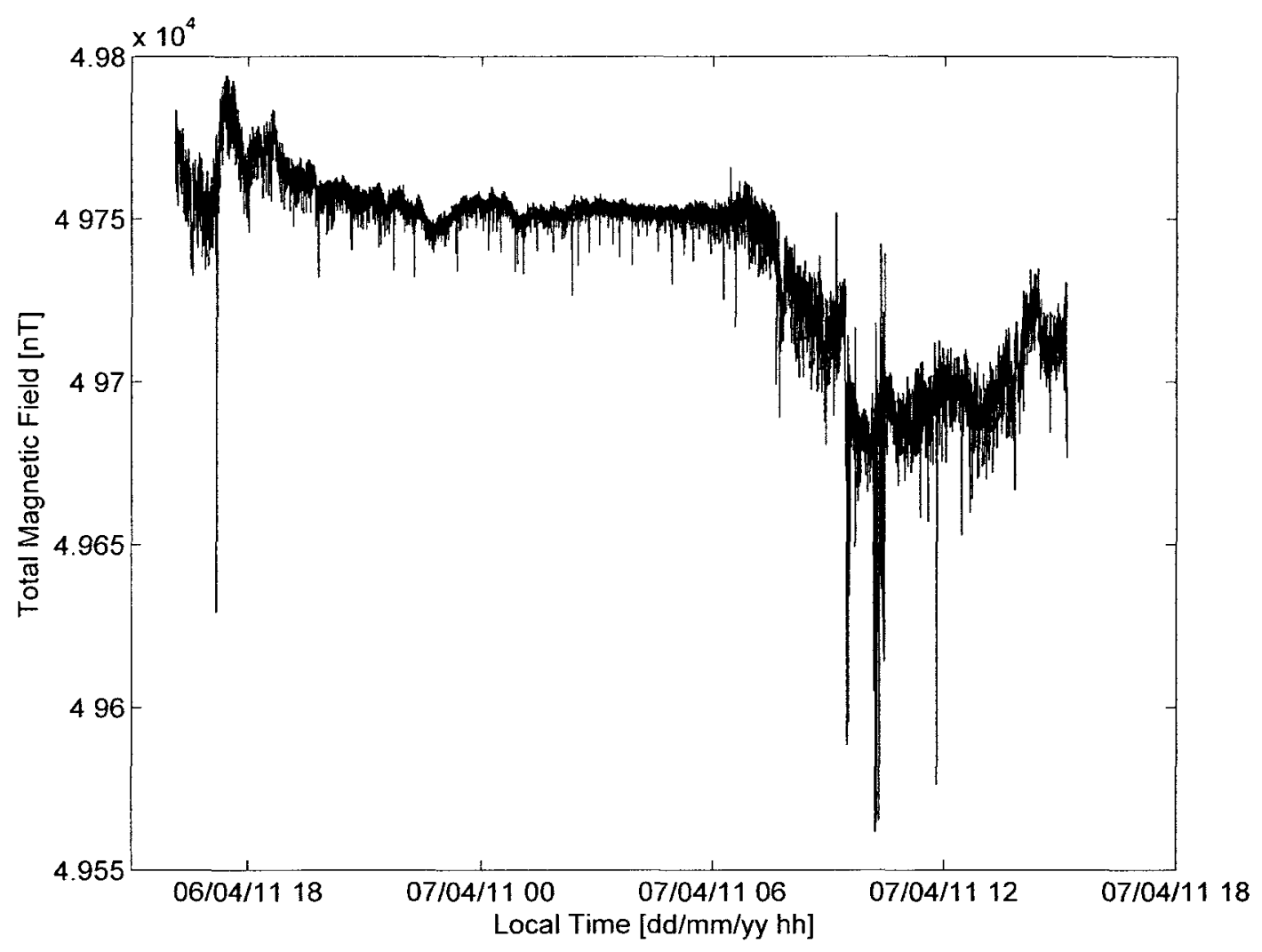

Figure 3.3: Magnetic field behaviour at SGL Headquarters over the course of a day. Measurements were taken from 06 April 2011 at 16:07 EST to 07 April 2011 at 15:39 EST. The maximum variation during off-peak hours is $\approx 25 \mathrm{nT}$, with a daily variation of $\approx 200 \mathrm{nT}$.

\section{Geomagnetic Laboratory}

The majority of testing was conducted at the Geomagnetic Laboratory of Natural Resources Canada which is located approximately $11 \mathrm{~km}$ east of the Carleton University campus. The facility is in a location that is negligibly affected by the magnetic noise generated by the City of Ottawa, and is located on a 30 hectare compound containing 16 buildings of nonmagnetic construction. All of the indoor testing was completed in Building 4, which is also referred to as the Absolute Building, which is shown in Figure 3.4. The Absolute Building is used for baseline calibration of 
magnetic field measurements for the Ottawa observatory as part of the International Real-time Magnetic Observatory Network (INTERMAGNET).

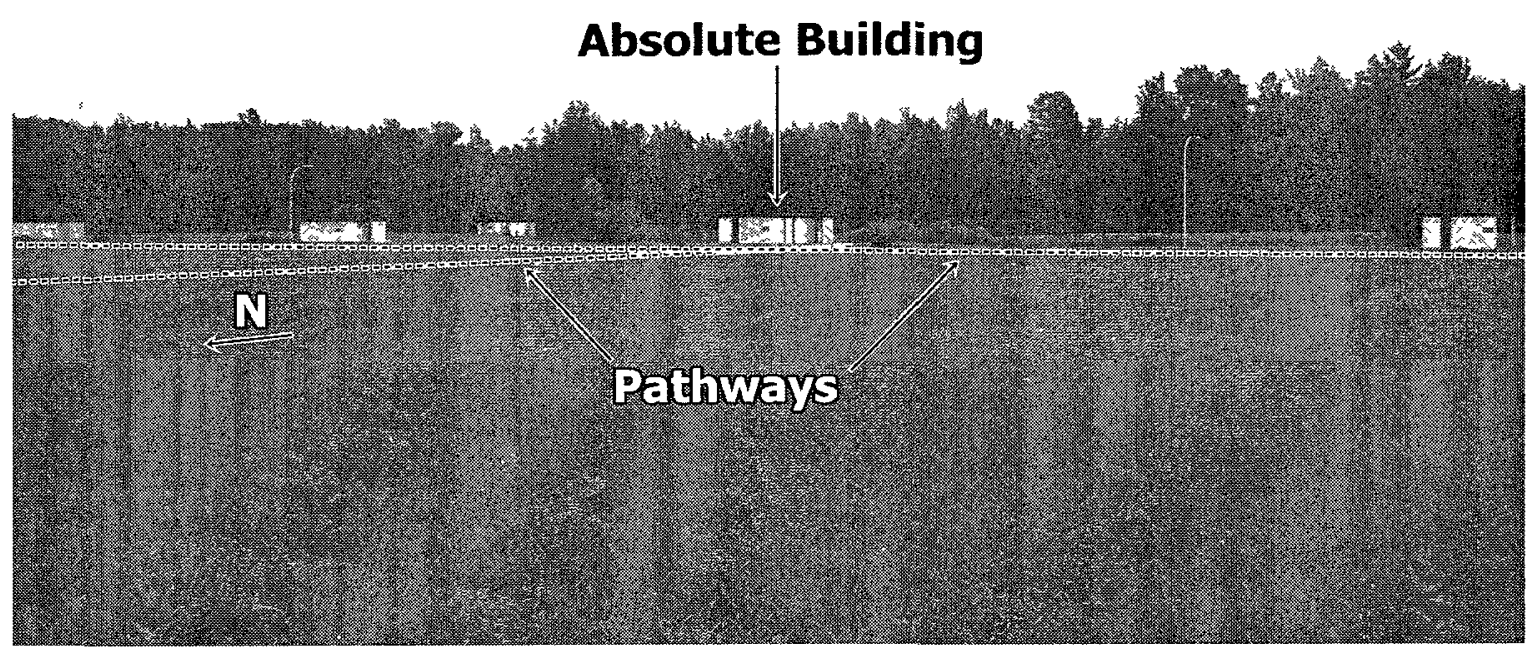

Figure 3.4: Absolute building and surrounding pathways at the Geomagnetic Laboratory

Outdoor testing was completed on the pathways surrounding Building 4, with the exception of the powered engine test which was conducted near the Calibration Building. The Geomagnetic Laboratory was proven to provide an environment with minimal magnetic field gradient and only naturally occurring variations in the background field, allowing for the completion of accurate magnetic signature testing. The daily field variation may be seen in Figure 3.5 . 


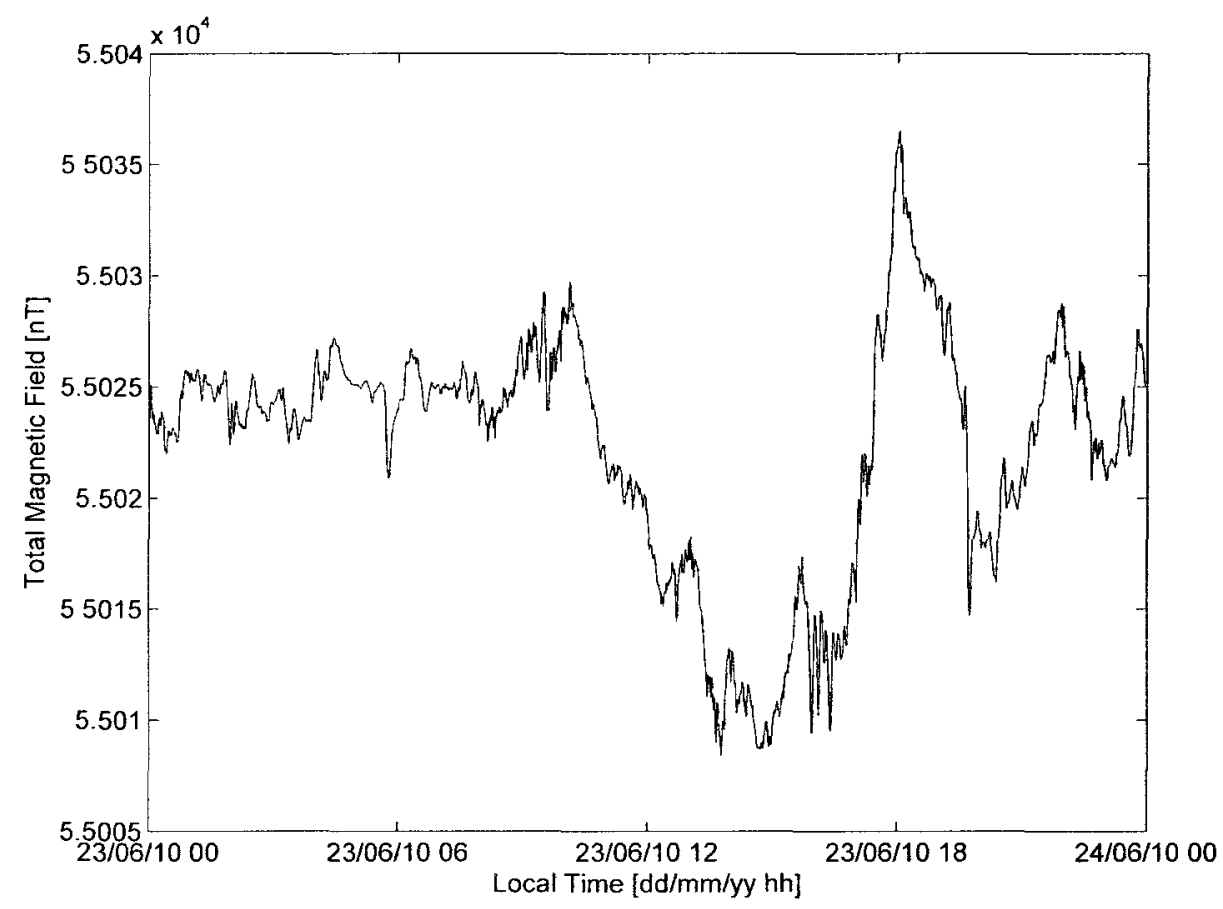

Figure 3.5: Magnetic field behaviour at the Geomagnetic Laboratory over the course of a day]. Measurements were taken from 23 June 2010 at 00:00 UTC to 23 June 2010 at 23:59 UTC. The maximum variation during off-peak hours is $\approx 10 \mathrm{nT}$, with a daily variation of $\approx 25 \mathrm{nT}$. Data collected at the Ottawa INTERMAGNET Observatory (www. intermagnet.org).

\subsection{GeoSurv II Magnetic Measurement System}

GeoSurv II is designed to carry one or two Geometrics G-822A optically pumped Cesium magnetometers and one Billingsley TFM100 triaxial fluxgate magnetometer. Both magnetometers will be briefly described in this section.

\subsubsection{Optically Pumped Cesium Magnetometer}

Optically pumped Cesium magnetometers measure the total field in high resolution on the order of $0.01 \mathrm{nT}$. Cesium magnetometers are sensitive to orientation and will only operate in certain orientations relative to the local magnetic field. They are the 
magnetometer of choice for geomagnetic surveying since they allow for high resolution measurements at high frequencies, with inherently low noise [13]. The Geometrics G822A optically pumped Cesium magnetometer is shown in Figure 3.6.

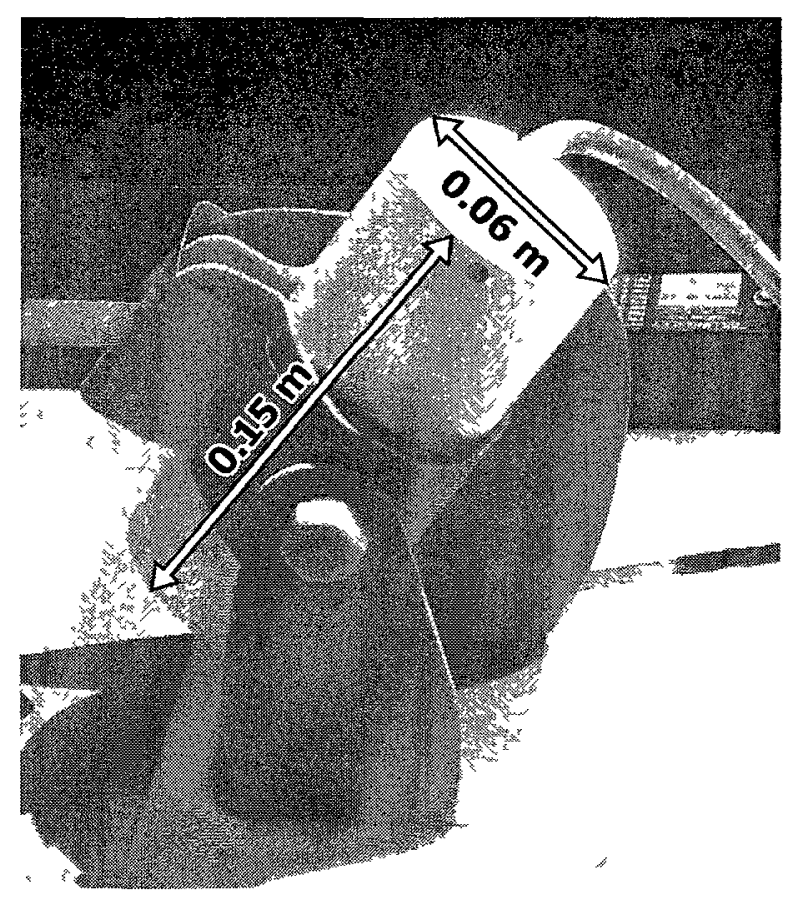

Figure 3.6: Geometrics G-822A optically pumped Cesium magnetometer.

Optically pumped Cesium magnetometers operate by exciting Cesium vapour atoms using light of a proper wavelength. This causes the Cesium atoms to jump to a higher energy level. The excited Cesium atoms then reemit energy in the form of a photon, and drop to a lower energy state than they originally occupied [28]. When all of the Cesium atoms within the vapour cell are in this lower energy level, the sample no longer absorbs light and is said to be polarized. An alternating magnetic field of varying frequency is then applied until the Cesium atoms return to their original energy level which is detected by the absorption of light, at which point the process starts again [13]. The local magnetic field will dictate the energy level of the polan ized state. Hence, the frequency of the applied magnetic field that corresponds 
to the point at which the Cesium atoms return to the original energy level, known as the Larmor precession frequency, may be correlated with the magnitude of the local magnetic field.

\subsubsection{Fluxgate (Saturable-Core) Magnetometer}

Fluxgate magnetometers are used to measure the vector magnetic field in the range of approximately $10^{-10}$ to $10^{-4} \mathrm{~T}$. They are best suited for measurements which have a desired resolution in the $1 \mathrm{nT}$ range. Fluxgate magnetometers are composed of a magnetically susceptible core wrapped with two separate wire coils, an excitation coil and a sense coil. An alternating current is passed through the excitation coil, magnetizing the core. The magnetization of the soft core will change with the polarity and magnitude of the excitation voltage, as well as with the local magnetic field. The alternating magnetic field produced by both the excitation coil and the core induces a current within the sense coil. which can then be correlated to the magnetic field magnitude acting along the direction of the two coils [28]. The use of three orthogonal (triaxial) coils allows for the measurement of all vector components of the magnetic field. The Billingsley TFM100 triaxial fluxgate magnetometer is shown in Figure 3.7.

Fluxgate magnetometers are inexpensive compared to other magnetometer technologies, as well as being rugged, reliable and compact. Furthermore, they contain no moving parts, and are temperature stable [28]. Applications of fluxgate magnetometers include aircraft and vehicle navigation, geophysical prospecting, and detection of ferromagnetic objects. 


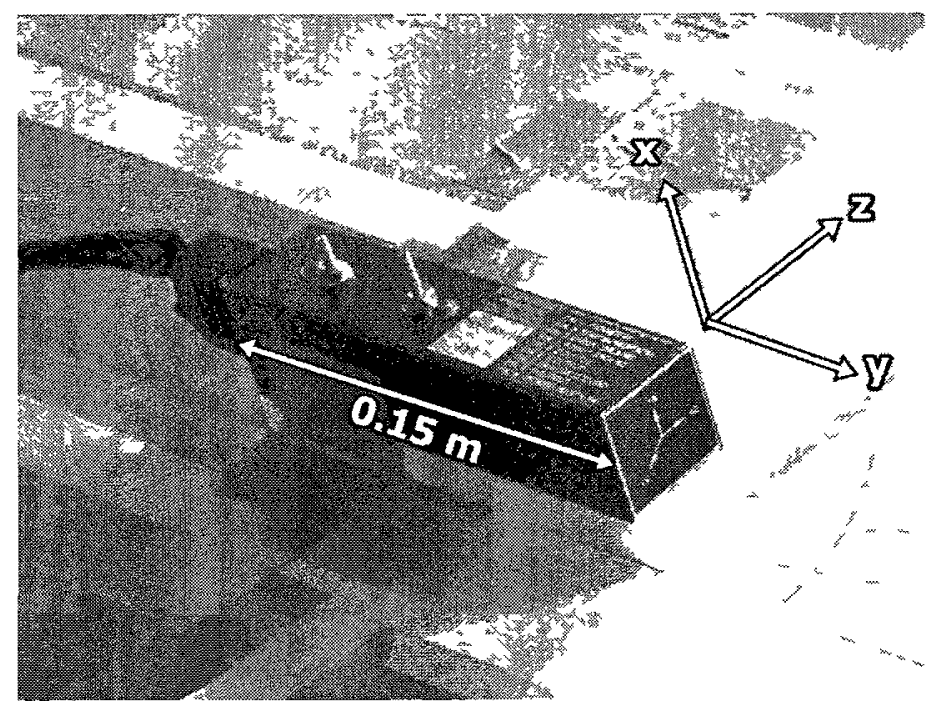

Figure 3.7: Billingsley TFM100 triaxial fluxgate magnetometer.

\subsubsection{Sensors Used for Testing}

The sensors and data acquisition system used for testing were supplied by SGL. The sensors used were the Geometrics G-822A optıcally pumped Cesium magnetometers and the Billngsley TFM100 triaxial fluxgate magnetometer, the same sensors that will be on the final GeoSurv II design Most expermments involved the use of at least two sensors.

Additıonally, a Foerster Magnetoscop was used to locate magnetic components. The magnetoscop utilizes a single uniaxıal fluxgate to measure local magnetic fields. It is a hand-held unit with a display which indicates the deviation in the local background field caused by a component The specific configuration of sensors used during testing will be outlined for each test procedure in Chapters 4 and 7 .

A sensitivity analysis was conducted to determine the optimum sample hold time for static experiments by recording 12 one minute long intervals at a sampling frequency of $160 \mathrm{~Hz}$. The one minute long intervals were further broken down into samples 
whereby measurements were defined as the first $N$ seconds of each interval, where $N=1,2,3,4,5,10,15,20,25,30,40,50$, or 60 seconds. The standard deviation was taken for each sample hold time and averaged over the 12 intervals; the samples were not corrected for the changes in the background magnetic field. The results show that the sample standard deviation increases with hold time, as can be seen in Figure 3.8. For this reason, it was decided to keep static measurement intervals to 5 seconds or less.

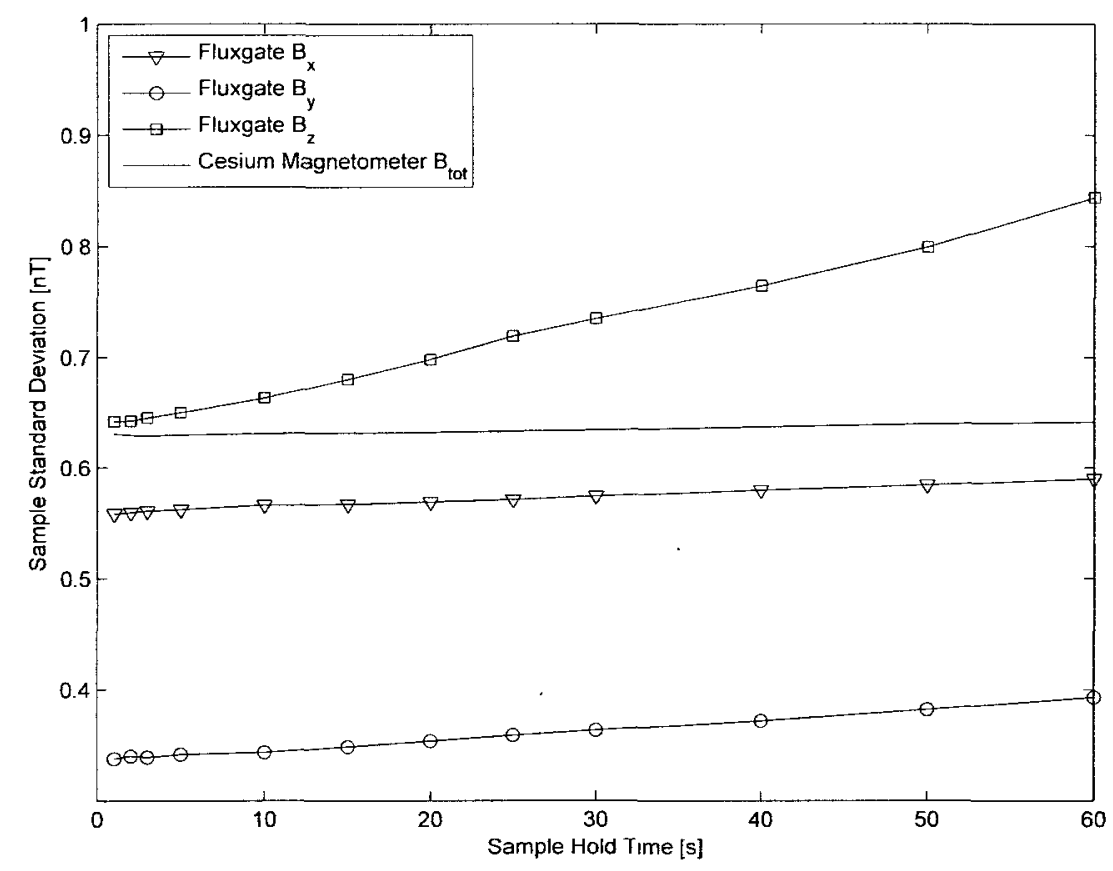

Figure 3.8: Sensitivity analysis for sensor hold time. The $B_{x}, B_{y}$, and $B_{z}$ measurements are for a Billingsley TFM100 triaxial fluxgate magnetometer. and the $B_{\text {tot }}$ measurement is for a Geometrics G-822A optically pumped Cesium magnetometer. The results were not corrected for changes in the background field. 


\section{Chapter 4}

\section{Testing and Analysis of Components of GeoSurv II Prototype}

\subsection{Introduction}

This chapter details a thorough investigation of the magnetic signature of individual components on the GeoSurv II prototype. This investigation was necessary to locate and identify the components which have the greatest contribution to the wingtip magnetic noise. Three main contributors were identified early in the investigation. They are, in order of significance, the servo actuators, the engine and engine assembly, and the avionics package. All experiments listed in this chapter are explained in greater detail in [29].

\subsection{Experimental Procedure and Analysis Meth- ods}

There are three experimental methods that were used for the majority of the component testing: magnetic component localization, rotational testing, and gradiometer testing. All three will be described in this section. As well, common equations used 
for the data analysis are presented. In this chapter, any deviations from these experimental procedures and use of analysis equations will be noted in the respective sections.

\subsubsection{Magnetic Component Localization}

Before beginning component testing it was necessary to identify the components with the largest magnetic signature, so that they could be given greater attention. A Foerster Magnetoscop was used to identify problem components. As was discussed in Section 3.3.3, the Foerster Magnetoscop utilizes a single uniaxial fluxgate to measure local magnetic fields. Since it is handheld, it allows for the quick identification of magnetic components. Alternatively, magnetic components may also be located using permanent magnets which are attracted to other permanent magnets and ferromagnetic material.

The magnetic field effect at a set distance was measured for each component using the Foerster Magnetoscop. From this investigation, a summary of problem components was produced, allowing for a prioritized list of problem components to be created, as may be found in [30].

\subsubsection{Rotational Testing Procedure}

Rotational testing involves measuring the magnetic field at a fixed point for an object at a known distance that is being rotated in a controlled manner. As was covered in Section 2.3 , the total magnetic field of a dipole will vary between $\left\|\vec{B}_{\max }\right\|$ and $0.5\left\|\vec{B}_{\max }\right\|$ for when the magnetic moment vector is most closely aligned with and normal to the position vector, respectively. Rotational testing seeks to take advantage of this behaviour. 
This testing requires the use of two magnetometers; one as a measurement sensor and the other as a reference sensor. The general procedure is illustrated in Figure 4.1 , and is conducted as follows:

1. Place the measurement sensor on the measurement platform, and level the platform.

2. Place the reference sensor far enough away from the measurement platform so that it measures only variations in the background magnetic field. If using vector magnetometers, align the reference sensor with the measurement sensor.

3. Place the test component far enough away from both sensors such that neither sensor is affected. Make a record of the readings at both magnetometers to establish a baseline without the component present.

4. Set the component on the test platform at a known orientation and distance relative to the measurement magnetometer. Take a measurement.

5. Rotate the component by some incremental angle about one of its geometrical axes at some defined fixed point. Record the magnetic field values at this orientation.

6. Repeat step 5 until 360 degree rotation is completed. It is beneficial to repeat step 3 at least once throughout the rotation if the experiment takes longer than roughly 10 minutes. 


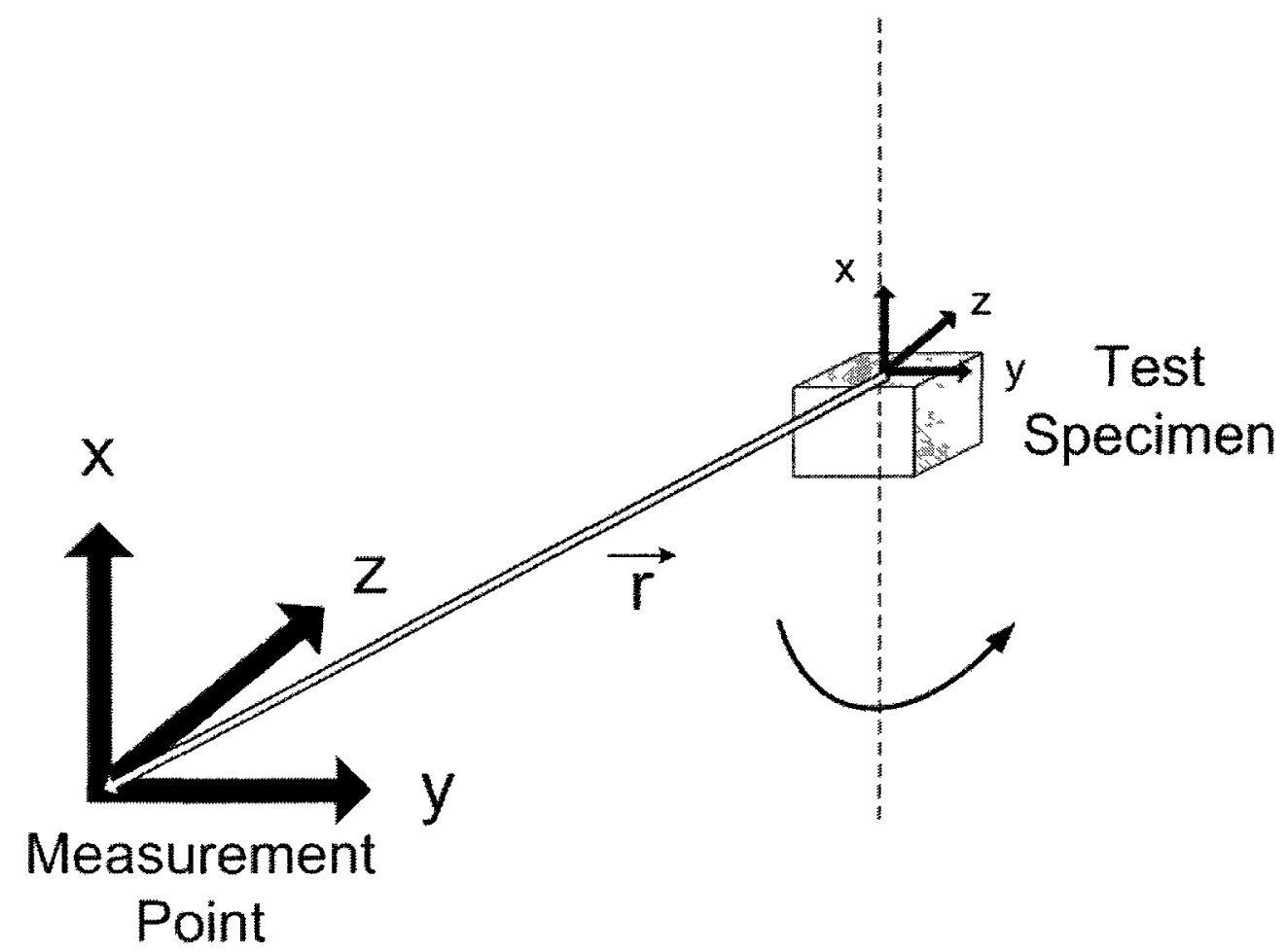

Figure 4.1: Illustration of rotation test used during testing. Test component is rotated about one of its geometrical axes at a known fixed point. Measurements are taken at incremental angles spanning a full rotation.

The rotation test may be completed for rotations about more than one component axis. Also, a custom nonmagnetic experimental apparatus is needed to maintain control over position vectors and component orientations.

\subsubsection{Gradiometer Testing Procedure}

The objective of gradiometer testing is to measure the behaviour of the magnetic field with distance from two or more magnetometers arranged in a gradiometer configuration. The gradiometer configuration allows for additional detail about the magnetic field that cannot be obtained from a single magnetometer. The data is useful for determining the magnitude of the magnetic noise effect of a component. and may be used for characterization. The general trend for this type of testing results in near 
zero magnetic field effect at large source-sensor distances. with the greatest magnetic field effect corresponding to points with the smallest source-sensor position vector for each dipole.

Ideally, this testing is completed with three magnetometers (two for measurement, one for reference), but may be completed with two magnetometers if the background magnetic field does not change substantially during the experiment. The general procedure is illustrated in Figure 4.2, and is conducted as follows:

1. Place measurement sensors close to but not obstructing the translational path of the component. Level and align sensors relative to each other.

2. If using a reference sensor, place far enough away from the translational path so that it is measures only variations in the background magnetic field. If using vector magnetometers, align the reference sensor with the measurement sensors.

3. Place the test component far enough away from all sensors such that no sensor is affected. Make a record of the readings at all magnetometers to establish a baseline without the component present.

4. Set the component at some known location on the translational path, aligning it in the desired orientation. Take a measurement.

5. Maintaining the same orientation, move the component along the path by some known incremental displacement. Record the magnetic field values at this orientation.

6. Repeat step 5 until the desired distance has been reached. It is beneficial to repeat step 3 at least once throughout the experiment if it takes longer than roughly 10 minutes. 


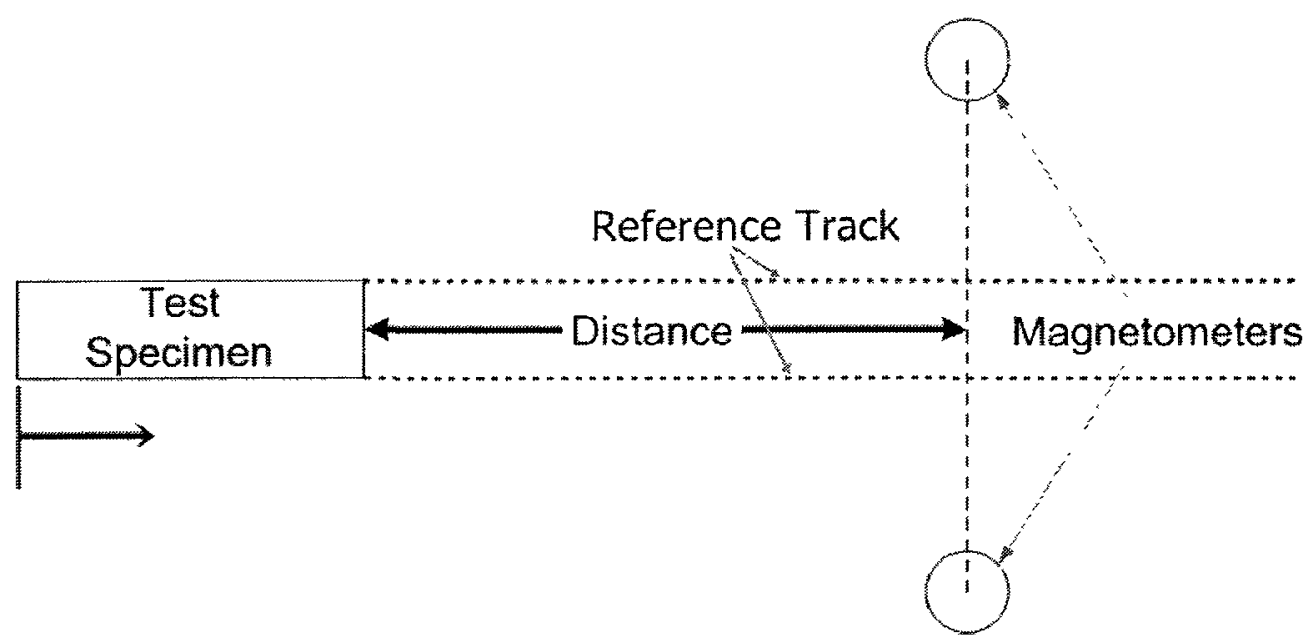

Figure 4.2: Illustration of gradiometer test used during testing. Test component is incrementally translated along a straight trajectory with stationary magnetometers located on both sides of the path at some fixed location. The fixed location is usually at the midpoint of the motion path.

Multiple translational axes relative to the component coordinate system may be used during gradiometer testing to provide additional data. A reference track must be defined for the component path, which can be accomplished using a variety of means. Distances from each sensor relative to the track must be measured and recorded to obtain proper position vectors. Additionally, if components must be mounted on a mobile test fixture to allow for easy translation. the test fixture must be nonmagnetic to avoid contaminating results.

\subsubsection{Analysis Methods}

This section outlines the methods most often used for experimental analysis. These methods are common for many of the experiments conducted, and will be referenced in the explanation of each individual experiment that they apply to. Analysis methods which are specific to certain tests will be presented with the corresponding experiments. 
For the majority of experiments conducted, a baseline reading was obtained to determine the background magnetic field without the test components present. This was useful to determine an offset, $\vec{B}_{i, o f f s e t}$, for magnetic field values. For experiments involving a reference sensor, the baseline may be defined by a single measurement of the magnetic field difference before the specimen was introduced to the measurement area. Alternatively, some analyses, usually involving experiments without a reference sensor, treated the baseline as the mean value of measurements taken without the specimen present at the beginning and end of each testing sequence, as calculated below:

$$
\vec{B}_{i, \text { of fset }}=\left(\vec{B}_{i, \text { baseline }, \text { start }}+\vec{B}_{i, \text { baseline }, \text { finish }}\right) / 2,
$$

where $\vec{B}_{i, b a s e l n n e, s t a r t}$ and $\vec{B}_{i, \text { baseline,finish }}$ are the baseline readings taken at the start and finish, respectively, of each testing sequence for magnetometer $i$. This approach is less accurate, therefore, in order to avoid larger baseline errors, it is suggested that experiments be well planned and executed within a short time.

The offset was removed from each measurement as follows:

$$
\vec{B}_{i, \text { offset removed }}=\vec{B}_{i, \text { measurement }}-\vec{B}_{i, \text { offset }},
$$

where $\vec{B}_{i, m e a s u r e m e n t}$ is the raw measurement value for magnetometer $i$. For analysis not involving a reference sensor, $\vec{B}_{i, o f f s e t r e m o v e d}$ is then treated as the true magnetic field effect for the measurement condition. If a reference sensor is utilized, changes in the background field may be calculated using (4.2), yielding $\vec{B}_{\text {ref, of fset removed, where }}$ $\vec{B}_{\text {ref;measurement }}$ is the magnetic field value during some measurement, and $\vec{B}_{\text {ref,of } f \text { set }}$ is the magnetic field during the baseline reading. both taken at the reference sensor. The background field changes may then be removed from each reading for measurement 
sensor $i$ using the following:

$$
\vec{B}_{i, \text { corrected }}=\vec{B}_{i, \text { of fset removed }}-\vec{B}_{\text {ref, of fset removed }},
$$

where $\vec{B}_{i, \text { offset removed }}$ and $\vec{B}_{\text {ref,offset removed }}$ are the magnetic field effect values for measurement sensor $i$ and the reference sensor with offsets removed, respectively. (4.3) will correct for any changes in the background field occurring during measurements.

Gradiometer readings help to reveal additional detail about geological survey areas and components, and provide a means of measuring magnetic field gradients. To calculate gradiometer values, $\Delta \vec{B}$. the difference in corrected magnetic field effects between measurement sensors 1 and $2, \vec{B}_{1 . c o r r e c t e d}$ and $\vec{B}_{2, \text { corrected }}$ is taken as follows:

$$
\Delta \vec{B}=\frac{\vec{B}_{1, \text { corrected }}-\vec{B}_{2, \text { corrected }}}{d}
$$

where $d$ is the distance between magnetometer locations. This yields a value with units $n T / m$.

\subsection{Servo Actuator Testing}

The servo actuators on the current GeoSurv II prototype, the Hitec HSR-5990TG coreless digital robot servo, were identified as the main contributor of magnetic noise onboard the aircraft. For this reason, primary focus was given to the servo actuators. Three different configurations were studied, as defined in Figure 4.3. Additionally, rotational testing was completed for the Hitec HSM-7990TG servo, which was selected to replace all Hitec HSR-5990TG servos on GeoSurv II due to its increased capacity and suitability for unmanned aircraft. The coordinate system used for the Hitec HSM-7990TG servo was defined to be the same as for the Hitec HSR-5990TG, with 
the origin also at the geometric centre.

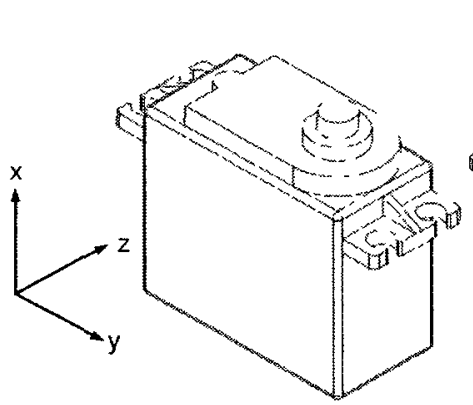

(a) Single

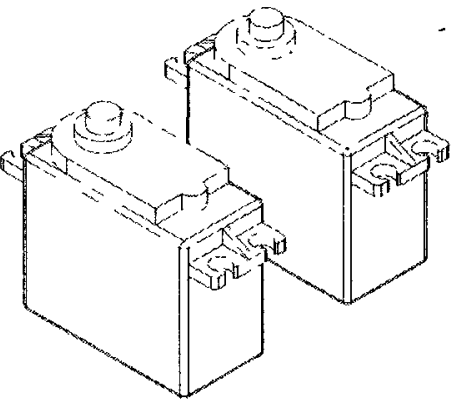

(b) Dual

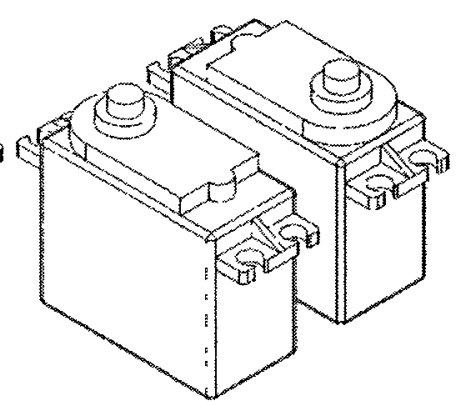

(c) Dual Counteroriented

Figure 4.3: Hitec HSR-5990TG servo configurations used during testing: (a)Single, (b)Dual, (c) Dual Counteroriented. The coordinate system for the servo is displayed in (a); the origin is at the geometric centre of the servo.

In addition to the testing mentioned in this section, a study similar to the one outlined by Yamazaki and Kawamoto [31] to determine the magnetic centre of the Hitec HSR-5990TG servo was completed. Knowledge of the location of the magnetic centre is necessary to determine position vector used for calculating the magnetic field effect of a source at some point in space. This test involved translating the servo between two magnetometers, and attempting to find the location at which the magnetic field effect was identical at both sensors. This process was repeated for movement along all servo axes, but yielded inconclusive results, which are outlined in $[29]$.

\subsubsection{Determination of Rate of Decay of Magnetic Field}

Magnetic fields will decrease in magnitude with distance from the source. The rate at which the magnetic field decreases with distance. rate of decay, is dependent on the nature of the magnetic field source. The objective of this experiment was to verify the rate of decay of the magnetic field effect for different servo configurations. 
As was covered in Section 2.3, the rate of decay of an ideally dipolar field will be proportional the inverse cube of distance. The experimental procedure used for this test is a variation of the gradiometer test outlined in Section 4.2 .3 which utilizes only a single measurement sensor, along with a reference sensor. Each servo configuration was moved at incremental distances away from the measurement sensor, along the $x$-, $y$-, and $z$ - servo configuration axes.

The data was analyzed using (4.1), (4.2). and (4.3) to remove the effect of the background field. As was expected, the rate of decay of the magnetic field for each servo was consistent with the behaviour of a permanent magnetic dipole. The results of this test may be seen in Figure 4.4 for the single, dual, and dual counteroriented configurations. 

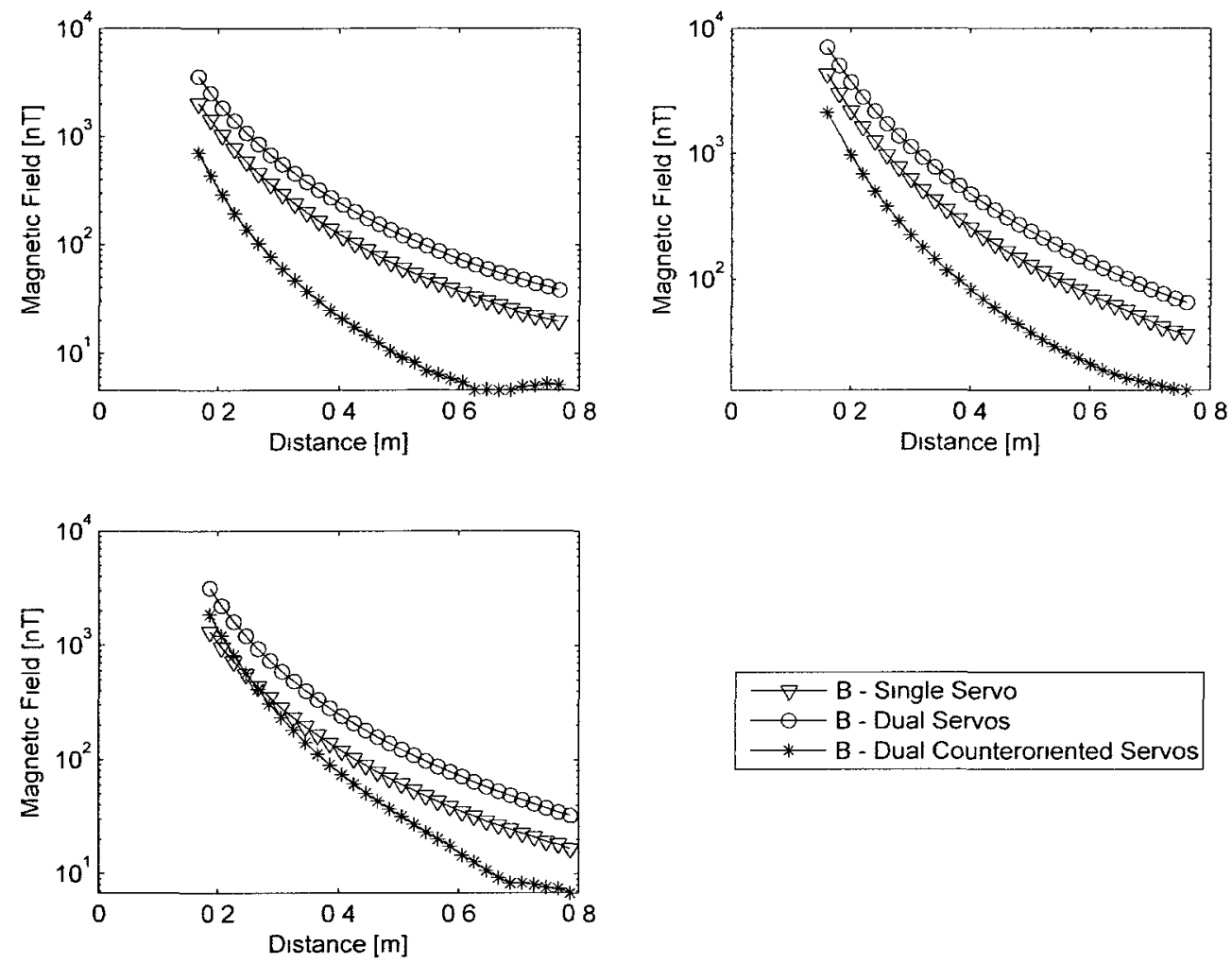

Figure 4.4: Magnetic total field rate of decay results for different Hitec HSR-5990TG servo configurations. Increasing distance along the $\mathrm{x}$ - (top left), $\mathrm{y}$ - (top right), and $z$ - (bottom) axis of a single servo. The dual servo configuration is seen to have the largest effect, and the dual counteroriented configuration has the smallest effect.

From Figure 4.4, it can be noted that the magnitude of the magnetic field is much larger for the dual servo configuration than for the other two configurations. Also interesting is that the magnitude of the magnetic field for the dual counteroriented servo configuration displays smaller magnetic field values than a single servo for two of the three directions. It can also be noted that for dual counteroriented servos, the $\mathrm{Y}$ component of the magnetic field along the $\mathrm{X}$ direction actually increases slightly in the near field region before it begins to decrease at a rate consistent with a magnetic dipole, as can be seen in the vector component plots in Appendix A, Figures A.1 
to A.3. This is due to the multipole effects of counterorienting the two servos. The results of this experiment reveal that the magnetic signature of servo pairs may be reduced by simply counterorienting each servo in the pair with respect to one another.

\subsubsection{Static Field Variations for Servos Under Rotation}

The primary experiment conducted on each servo was the rotation test described in Section 4.2.2. This procedure yields the most useful data for the characterization schemes outlined in Chapter 5. For this test procedure, two magnetic moment test apparatus (MMTA) were designed and constructed to allow for precise control of the position vectors and angles measured experimentally. The MMTA-1D allowed for rotation only about the x-axis of the Hitec HSR-5990TG servo at distances of 0.20 $\mathrm{m}, 0.25 \mathrm{~m}$, and $0.30 \mathrm{~m}$, and was used to collect preliminary data. The MMTA-1D is shown in Figure 4.5. 


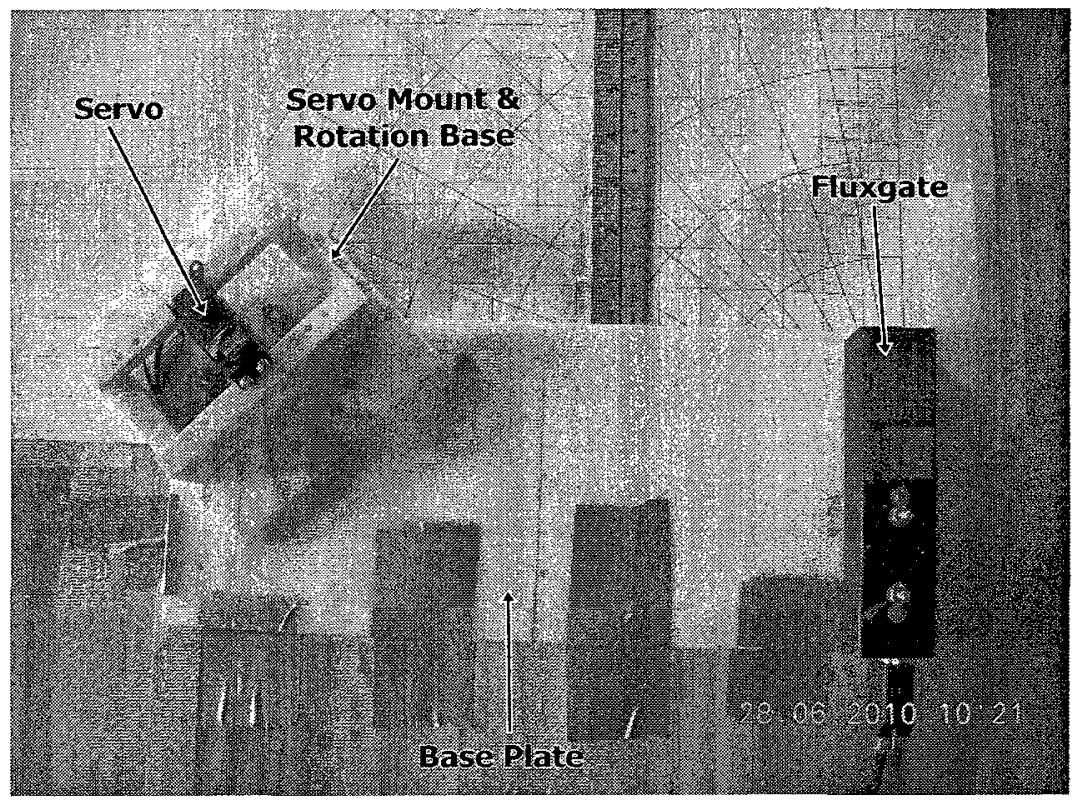

Figure 4.5: MMTA-1D test apparatus with servo. The servo is fastened to the servo mount which is fastened to the rotation base. The rotation base has holes drilled at $15^{\circ}$ intervals which are aligned using dowels to control the orientation and position vector of each measurement.

The MMTA-3D was an improvement on the MMTA-1D, allowing for rotations about all three axes of components smaller than $0.127 \times 0.127 \times 0.127 \mathrm{~m}$ at distances varying from $0.16 \mathrm{~m}$ to $0.64 \mathrm{~m}$ in 0.02 increments in the -z-direction from the fluxgate centre as referenced to the coordinate system in Figure 4.6a. The MMTA-3D is capable of measuring the magnetic field at 136 unique points around each component at each incremental distance. It is constructed from entirely nonmagnetic and nonconductive materials: high density polyethylene (HDPE), nylon, and ABS plastic. It was designed to be modular such that testing of any new specimen would only require the fabrication of a single component, the component mount. The MMTA-3D has four main components:

- Base Plate: The base plate was necessary to ensure that the source-sensor y- and $\mathrm{z}$-position vectors are controlled. It is a flat HDPE sheet with holes drilled along 
the $\mathrm{z}$-axis with $0.02 \mathrm{~m}$ separation intended to allow the component to be tested at varying distances. It also has mounting holes for fluxgate magnetometers.

- Support: The support is composed of the support base and the two support sides. The support base has holes at intervals of $15^{\circ}$ at a radius of $0.06 \mathrm{~m}$. This allows measurement of the magnetic field about the specimen. The support sides, along with the thickness of the support base, provide the constant $\mathrm{x}$ position vector that is used during testing. The sides have mounting holes for the frame.

- Frame: The frame was constructed such that it can fit within the support in six different configurations $(+\mathrm{x},-\mathrm{x},+\mathrm{y},-\mathrm{y},+\mathrm{z},-\mathrm{z})$. It includes mounting holes for the component mount.

- Component Mount: This piece holds the component being tested, and is specific to individual components. It must be designed such that the geometrical centre of the component is aligned with the centre of the frame. It may be manufactured using a rapid prototyping machine. 


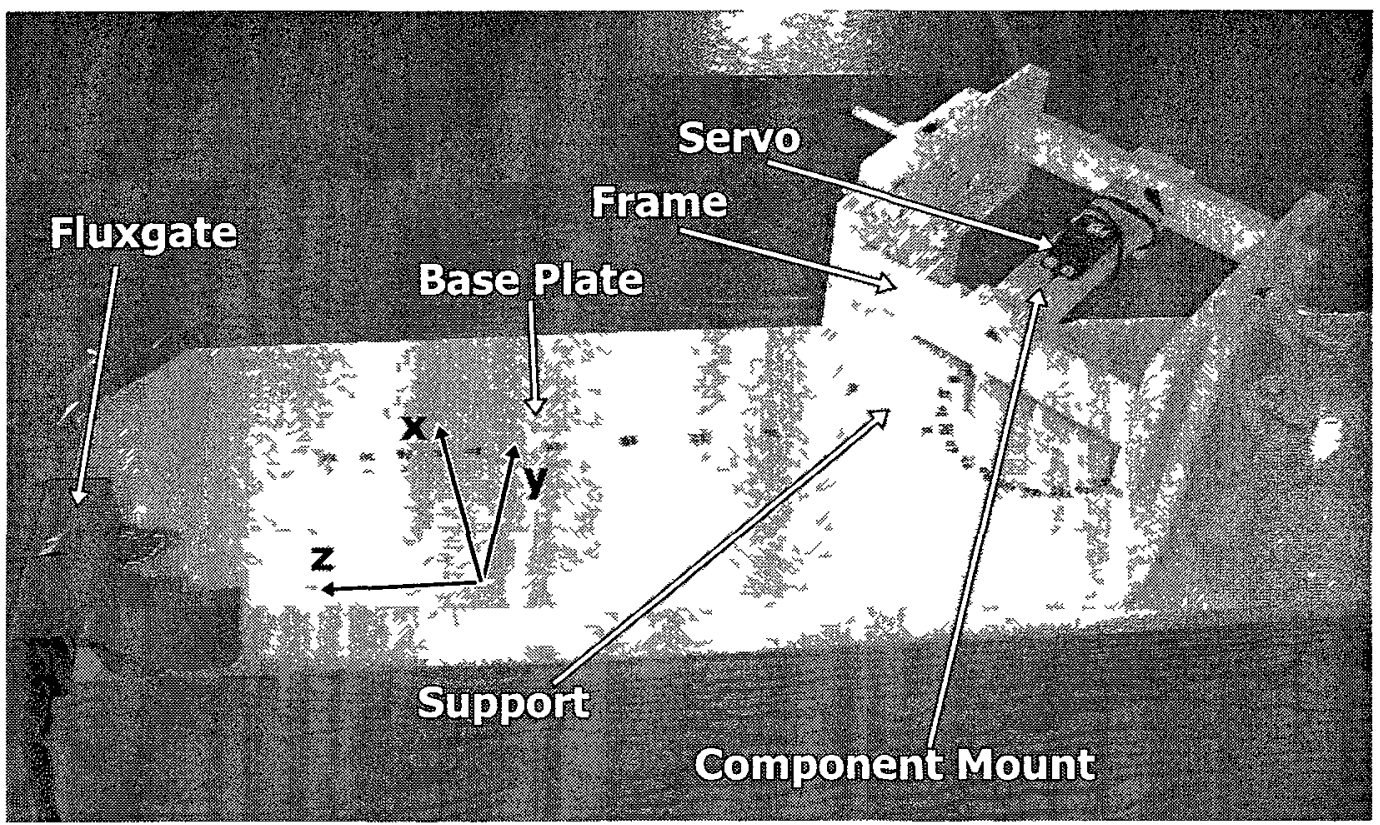

(a) x-axis rotation configuration

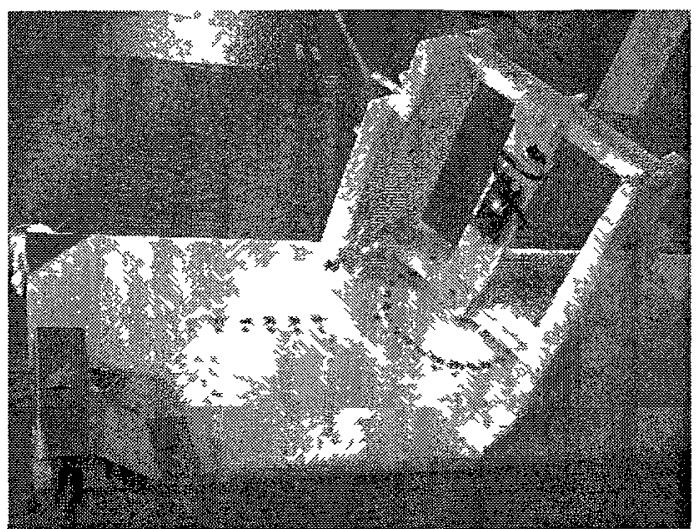

(b) y-axis rotation configuration

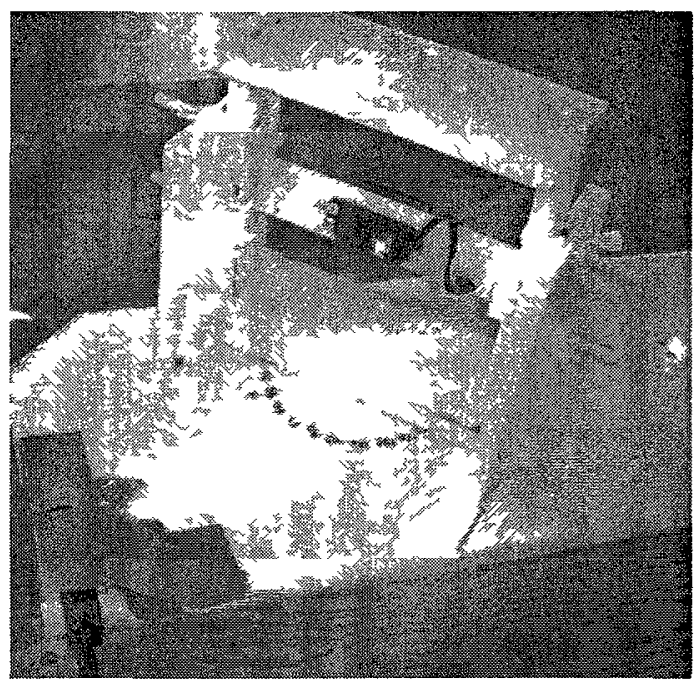

(c) z-axis rotation configuration

Figure 4.6: MMTA-3D test apparatus with servo in different configurations The support has holes drilled at $15^{\circ}$ intervals which are aligned using dowels to control the orientation and position vector of each measurement

The test procedure for this experiment follows the rotation test outlined in Section 422 Measurements were taken in $15^{\circ}$ increments, and baseline readings were taken before the $0^{\circ}, 90^{\circ}, 180^{\circ}, 270^{\circ}$, and after the $345^{\circ}$ measurements A reference 
sensor was utilized, so the true magnetic measurement values were obtained using (4.1), (4.2), and (4.3). The results of this test may be seen in Figures 4.7 for the MMTA-1D apparatus, and in Figures 4.8 and 4.9 for the MMTA-3D apparatus. The data shown in Figures 4.8 and 4.9 is useful for characterization strategies since it provides magnetic field readings at locations surrounding each servo. along different measurement planes.

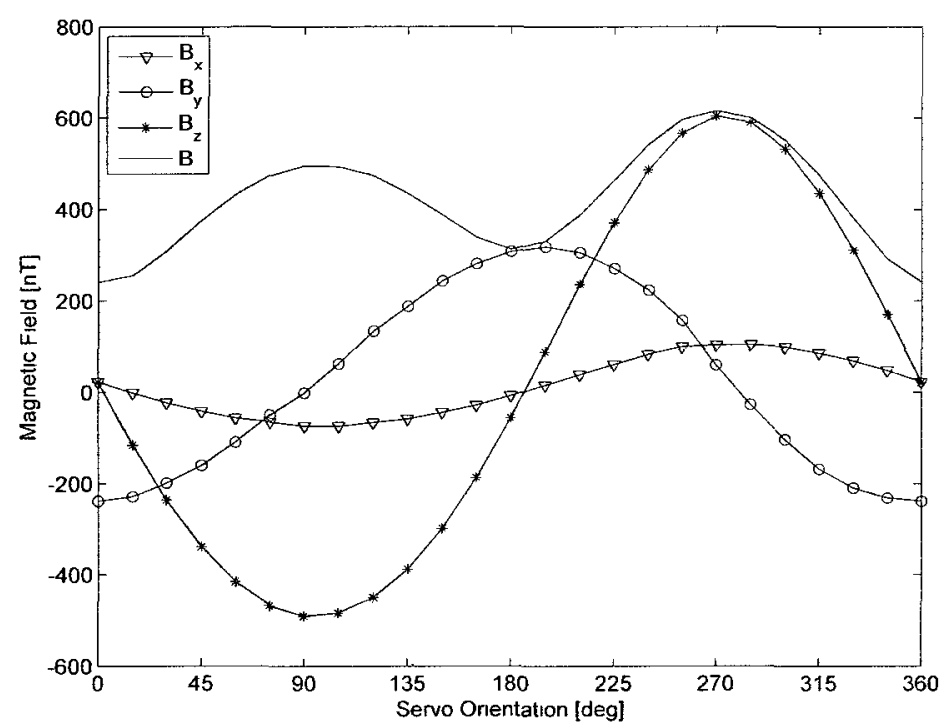

Figure 4.7: Magnetic field vs rotation for a single Hitec HSR-5990TG servo collected using MMTA-1D at a distance of $0.30 \mathrm{~m}$ in the $+\mathrm{z}$-direction of the measurement fluxgate. Shown are rotations about the $x$ - (top left), $y$ - (top right), and z-axis (bottom). The results show that the magnetic dipole moment is closely aligned to the $y$-axis of the servo. 

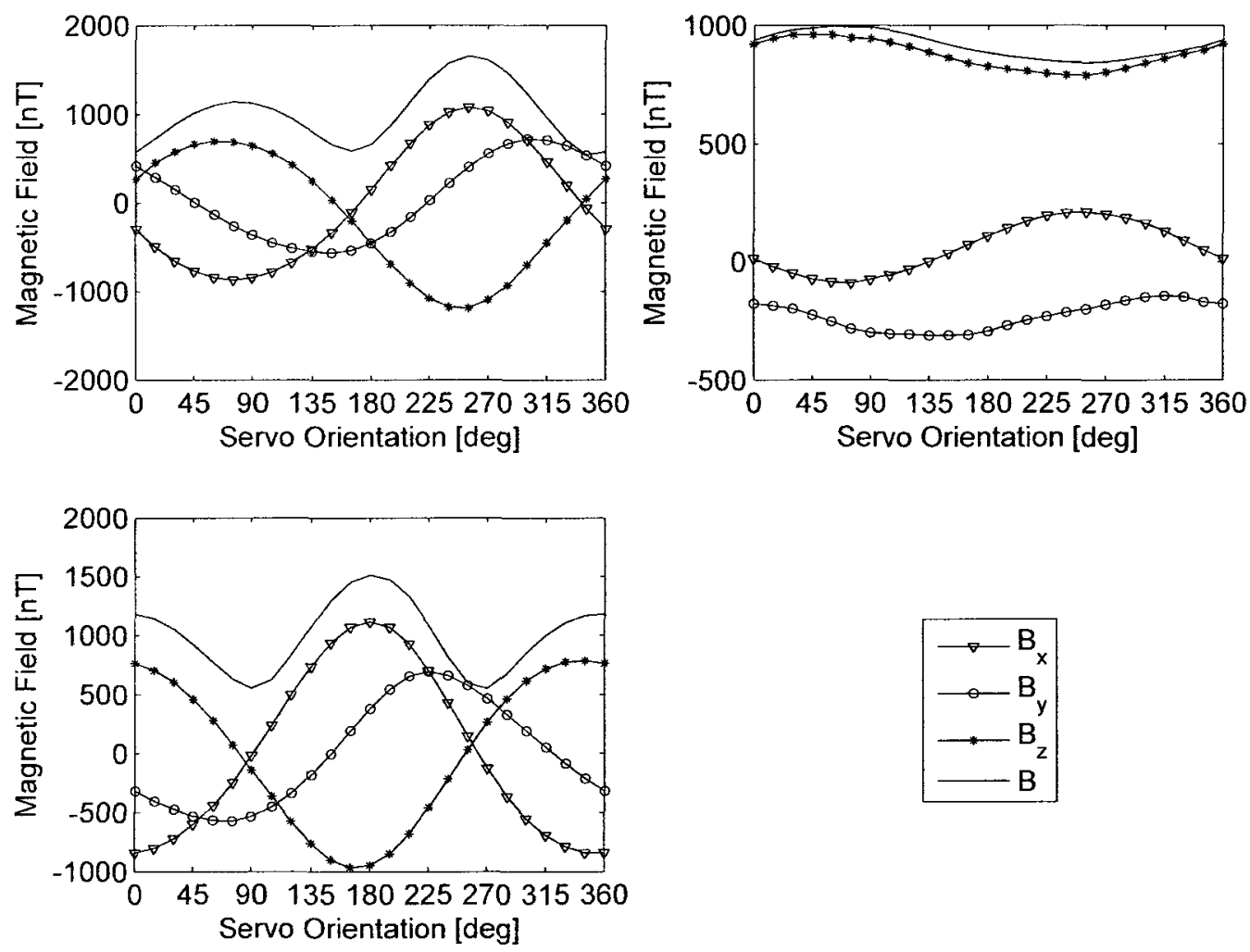

Figure 4.8: Magnetic field vs. rotation for a single Hitec HSR-5990TG servo collected using MMTA-3D at $0.22 \mathrm{~m}$ in the -z-direction of the measurement fluxgate. The results for rotation about the $y$-axis indicate that there is also a small $x$ component of the magnetic dipole moment. 

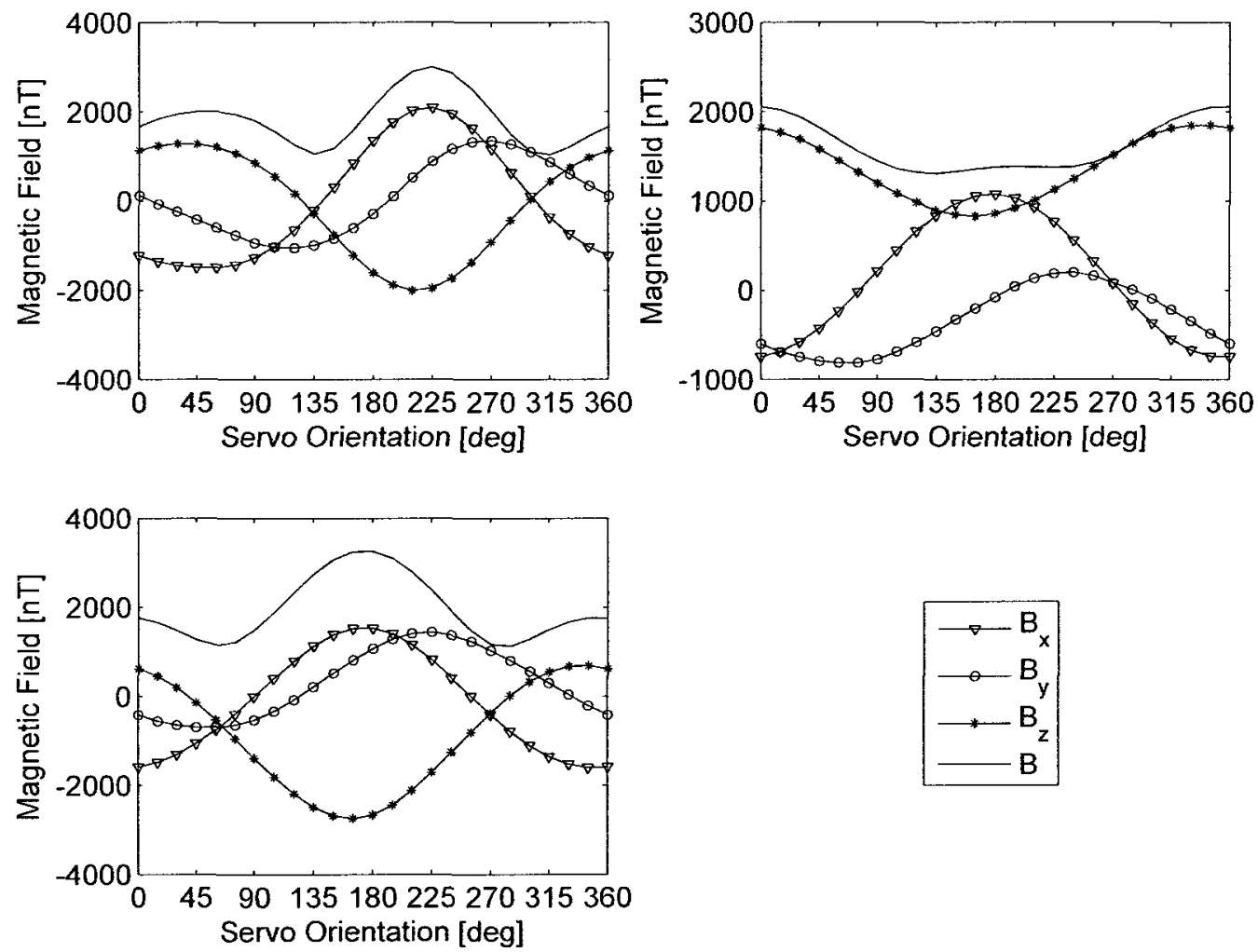

Figure 4.9: Magnetic field vs. rotation for a single Hitec HSM-7990TG servo collected using MMTA-3D at $0.22 \mathrm{~m}$ in the -z-direction of the measurement fluxgate. Shown are rotations about the $\mathrm{x}$ - (top left), $\mathrm{y}$ - (top right), and $\mathrm{z}$ - (bottom) axis. The magnetic signature of the Hitec HSM-7990TG is roughly.twice as large as that of the Hitec HSR-5990TG. 
From Figure 4.10, it can be noted that the dual servo configuration has an effect that is twice as large as that for a single servo. Additionally, counterorienting the $+y$-axis of each servo with respect to the other has a magnetic field effect which is less than that of a single servo. This is an important conclusion for the current GeoSurv II prototype since each control surface is controlled by a pair of servos. By reconfiguring all Hitec HSR-5990TG control surface servos such that each paired servo is counteroriented along the $+\mathrm{y}$-axis with respect to the other, the magnetic noise effect of the control surface servos can be reduced. Figures showing the magnetic field components for the dual and dual counteroriented servos may be found in Appendix A.

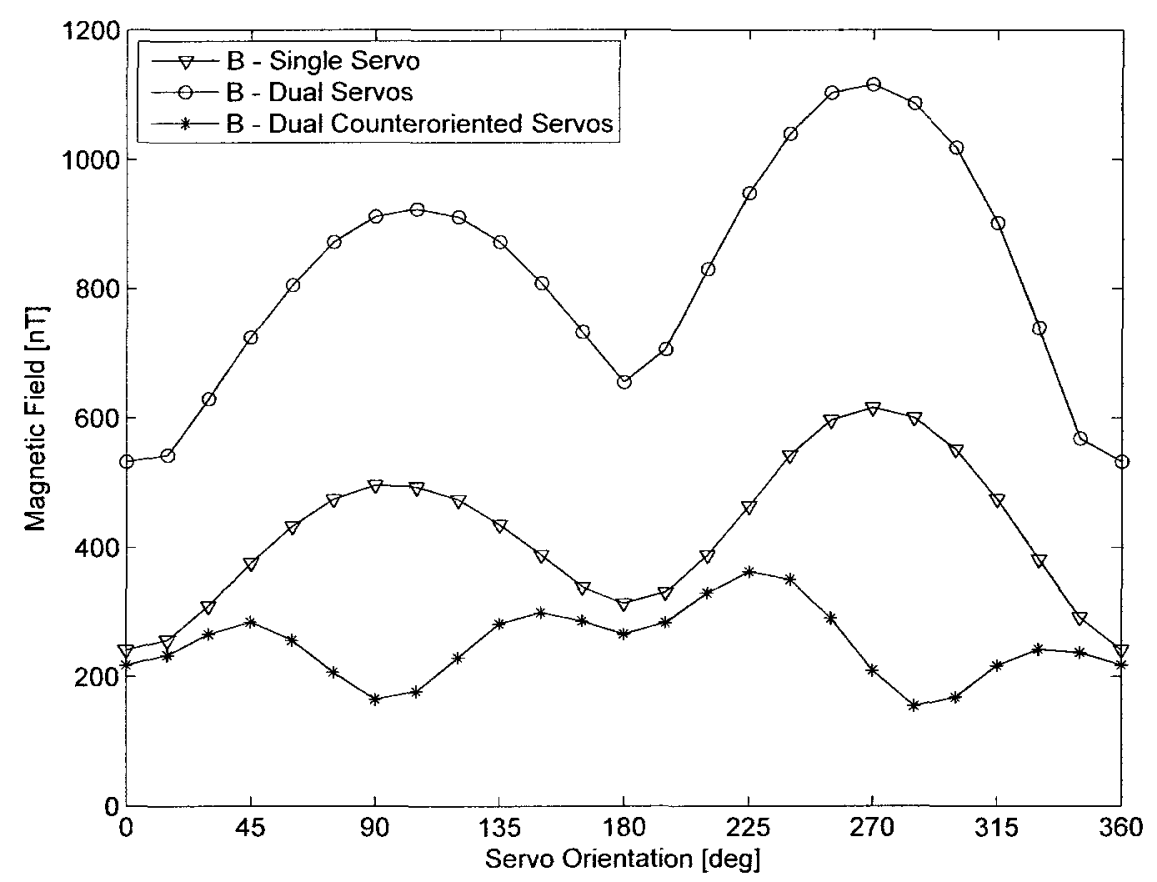

Figure 4.10: Magnetic field vs. rotation for three different Hitec HSR-5990TG servo configurations collected using MMTA-1D at $0.3 \mathrm{~m}$ in the +z-direction of the measurement fluxgate. Dual servo configuration has a signature that is twice that of a single servo. Dual counteroriented configuration has a lower signature than a single servo. 


\section{Servo Variability}

An investigation into the variability of the magnetic signatures for different Hitec HSR-5990TG servos was conducted using 13 servos from the GeoSurv II prototype. This investigation was necessary since it allows for servos with higher magnetic signatures to be positioned at locations further from the wingtip magnetometers. The variability was found using a mean magnetic signature calculated from the batch of 13 servos. The magnetic field value for the mean representative servo at each measurement point was calculated as follows:

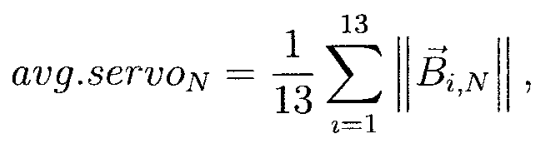

where $\left\|\vec{B}_{i, N}\right\|$ is the total magnetic field for servo $i$ at measurement point $N$, calculated at 12 measurement points using (4.1), (4.2), and (4.3). The variability is then calculated as follows:

$$
\% \text { variability }=\underbrace{\max }_{\text {for } i=1 \rightarrow 13} \frac{1}{12} \sum_{N=1}^{12}\left(\frac{\|\left(\left\|\vec{B}_{i, N}\right\|-\text { avg.servo }_{N}\right) \|}{\text { mean servo } N} \times 100\right) .
$$

The magnetic dipole moment of all servos tested appears to be oriented in the same direction, but there is a $\pm 11 \%$ maximum variability in the magnitude from the mean magnetic signature of the batch. This variability was evaluated for rotation about each servo's $\mathrm{x}$-axis, and a source-sensor distance of $0.22 \mathrm{~m}$. A comparison of the total magnetic field behaviour with rotation for all 13 servos is shown in Figure 4.11. 


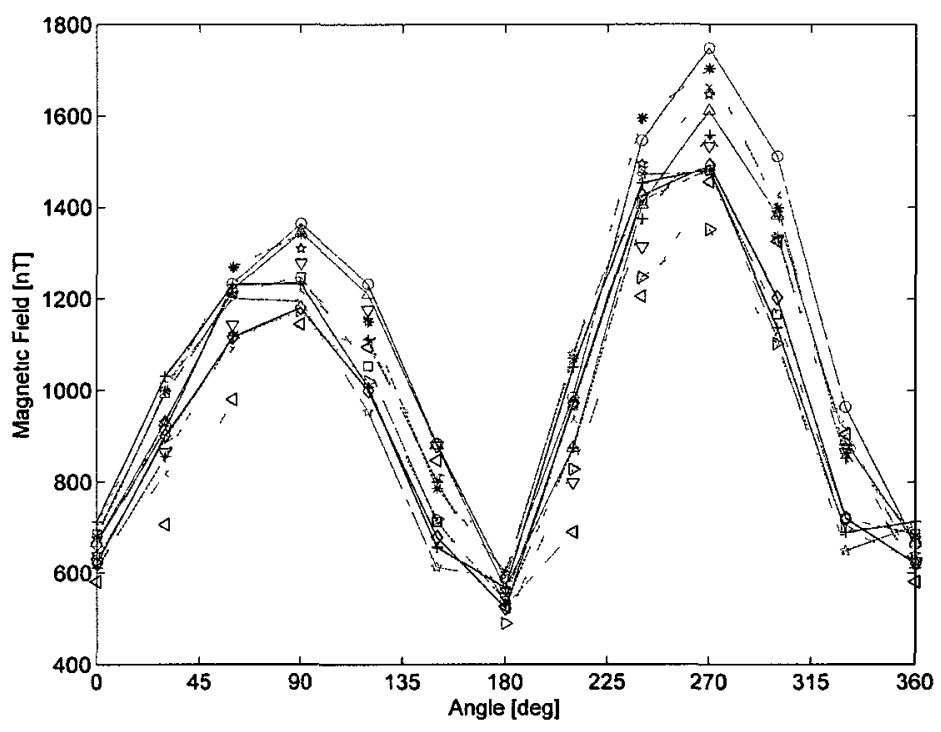

Figure 4.11: Variability in the magnetic signatures of 13 Hitec HSR-5990TG servos as shown for magnetic field vs rotation collected using MMTA-3D at $0.22 \mathrm{~m}$ in the -z-direction of the measurement fluxgate, rotating about the $\mathrm{x}$-axis of each servo. The orientation of the dipole moment vector is roughly the same for all servos, but the magnitude varies by $\pm 11 \%$ from the mean.

\subsubsection{Powered Servo Testing}

All powered servo testing was completed for the Hitec HSR-5990TG servo at a constant source to sensor distance without any rotation of the servo or sensor. The magnitude of the fluxgate-servo and Cesium magnetometer-servo position vectors were $0.822 \mathrm{~m}$ and $0.691 \mathrm{~m}$, respectively. Due the the minute magnetic field variations during powered testing, analysis was based primarily on the data collected from the Cesium magnetometers due to their better resolution as compared to fluxgate magnetometers. A simple estimation of the total magnetic field, $\|\vec{B}\|$ at some distance $r$, knowing the magnetic field, $\left\|\vec{B}_{\text {measured }}\right\|$, at some known distance, $r_{\text {measured }}$, may be extrapolated using the dipole approximation as follows:

$$
\|\vec{B}\|=\left\|\vec{B}_{\text {measured }}\right\|\left(\frac{r_{\text {measured }}}{r}\right)^{3}
$$


A baseline reading was taken before each test to be used as an offset. As well, a reference sensor was used to account for any variations in the background magnetic field.

Figure 4.12 shows the servo being powered at the $21 s$ mark, indicating a 0.06 $\mathrm{nT}$ difference in magnetic noise between the powered and unpowered states. This variation may be due to the servo arm rotating slightly to the preprogrammed zero position upon powering. As will be described in Chapter 5 , the minor variations in the servos magnetic field due to movement of the servo armature cannot be captured using characterization schemes since the variation is much smaller than the error associated with the curve fitting algorithm. Additionally, since the servos will always be powered during missions, they are approximated as time-invariant sources of magnetic noise. 


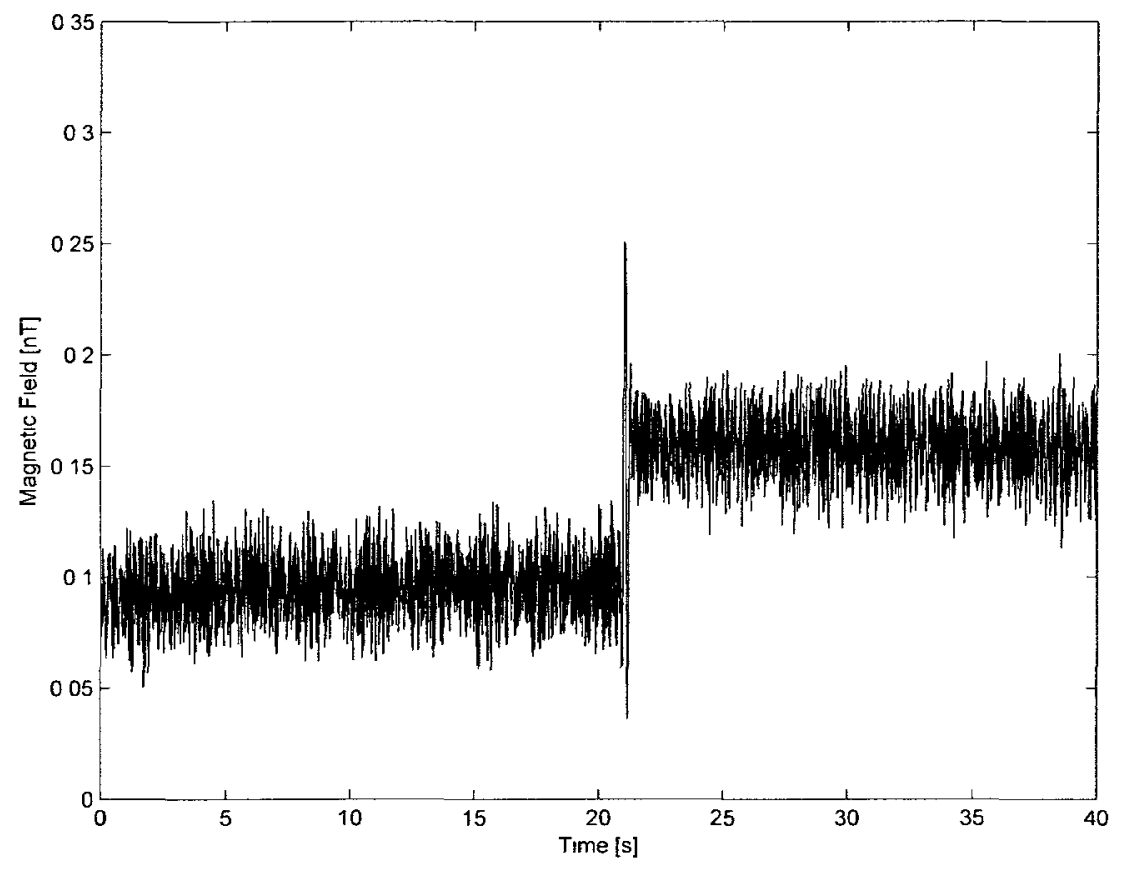

Figure 4.12: Behaviour of Magnetic Field while Powering a single Hitec HSR$5990 \mathrm{TG}$ servo. There is a $0.06 \mathrm{nT}$ increase in magnetic noise between the powered and unpowered states at a distance of $0.691 \mathrm{~m}$.

Full actuation of the servo led to a change in the magnetic field of approximately $0.2 \mathrm{nT}$. This value does have some variation from cycle to cycle. It can be seen in Figure 4.13 that actuating the Hitec HSR-5990TG servo with no load does cause a variation in the local magnetic field. It is important to recall that the sensor-source distance is $0.691 \mathrm{~m}$ for the cesium magnetometers, meaning that at the sensor-source distance of $1.5 \mathrm{~m}$ between the wingtip magnetometers and the flaperon servos, the effect is reduced to approximately $0.02 \mathrm{nT}$ calculated using (4.7). Additionally, if the servos are eventually moved to the fuselage, this effect will be even further reduced. 


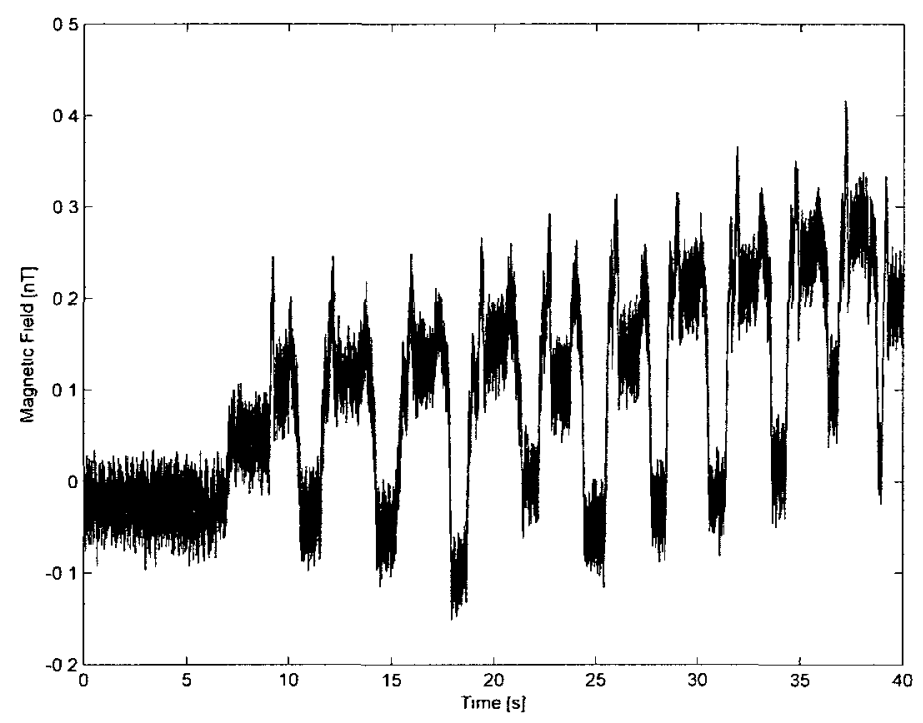

Figure 4.13: Behaviour of Magnetic Field while actuating a single Hitec HSR5990TG servo through its motion range. There is a $0.2 \mathrm{nT}$ variation in magnetic noise at a distance of $0.691 \mathrm{~m}$ due to actuation.

Comparing Figure 4.13 to Figure 4.14, it can be seen that manually actuating the servo has an effect that is of the same magnitude as the magnetic noise effect of actuating the servo under power. From this, it can be concluded that the variation in magnetic field due to actuating the servo is not due to the effect of current loops within the servo but is due to simply moving certain components of the servo. 


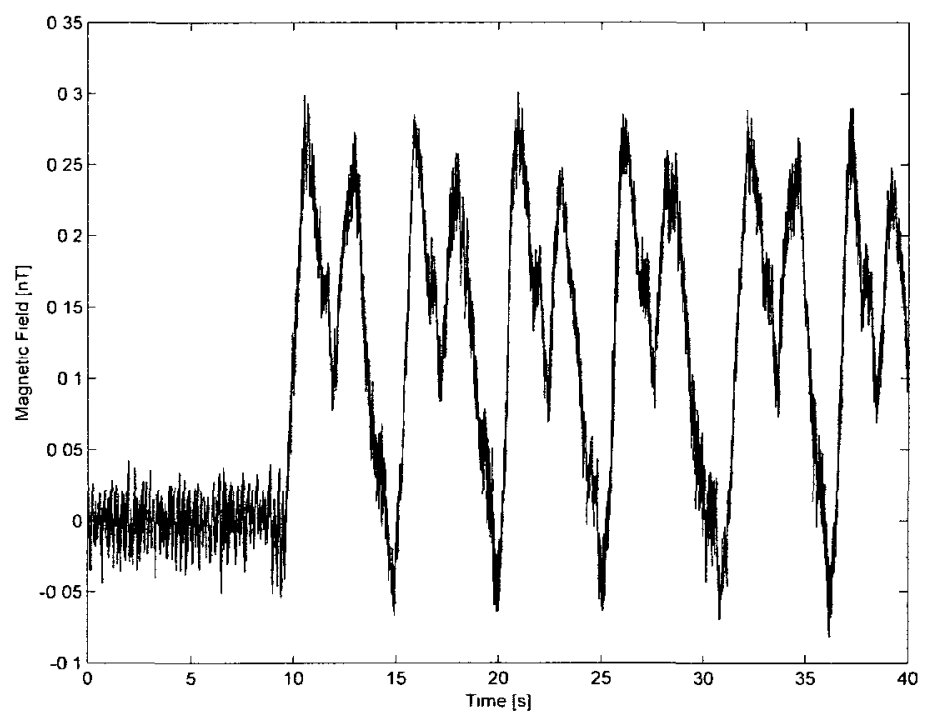

Figure 4.14: Behaviour of Magnetic Field while manually actuating a single Hitec HSR-5990TG servo through its motion range. The variation observed matched closely to that measured during powered actuation, leading to the conclusion that this effect is due to moving certain components of the servo instead of current loops within the servo.

Figure 4.15 shows the transient effect of a single Hitec HSR-5990TG servo cycled through different loading conditions. The performance of the servo under other loading conditions may be found in Appendix A. It can be seen that the sandbags used to load the servo affect the magnetic noise. Movement of the sandbags during experimentation produced an undesirable magnetic field effect which interfered with measurement of the true effect of the servo only. For this reason, the results require additional interpretation to remove the effect of the sand bags. The experimental procedure involved collecting data while cycling loaded and unloaded conditions, as well as applying a constant load for a short period of time. Additionally, the effect of the load itself was measured with the servos powcred off. 

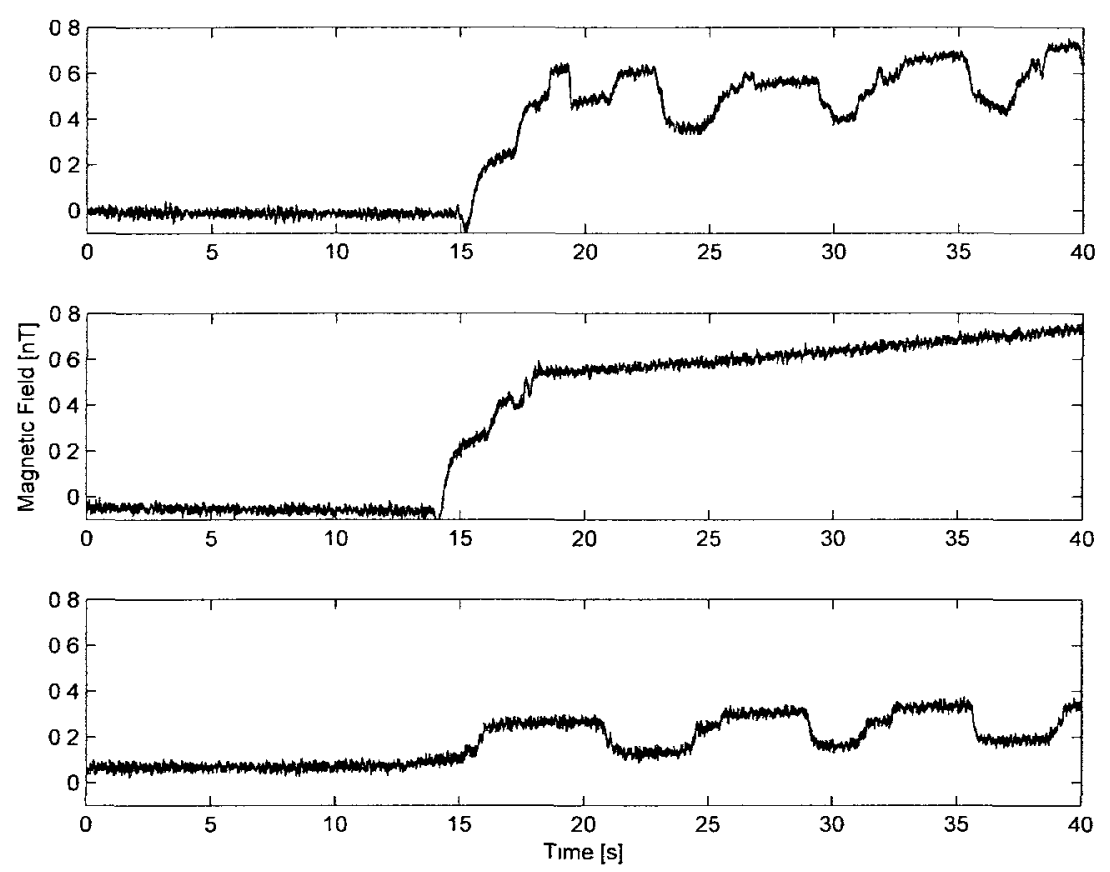

Figure 4.15: Transient effect of cycling a Hitec HSR-5990TG servo at load of approximately $19 \mathrm{~kg} \cdot \mathrm{cm}$. Shown are the results for cycling load/no load (top), constant load (middle), and the effect of the movement of the load alone (bottom). Sand bags were used for the load which had an adverse effect on the experimental results. It can be seen that the transient noise is roughly $0.4 \mathrm{nT}$ at a distance of $0.691 \mathrm{~m}$.

It is clear for the high load case, that applying a constant load causes a rise in total magnetic field of roughly $0.4 \mathrm{nT}$. Extrapolating this effect to predict the effect of a single flaperon servo at the wingtip magnetometer location using (4.7) yields a predicted transient effect of $0.04 \mathrm{nT}$. It is recommended that for future testing, loads utilizing water or other nonmagnetic materials be used to avoid contamination of the experiment. Additionally. it was noticed that the transient magnetic effect increases over time, possibly following hysteresis patterns. In conclusion, the magnetic field variation when experiencing high loads may be above design specifications, but can be further reduced by moving the flaperon servos away from the magnetometers. 


\subsection{Engine Testing}

The engine on GeoSurv II was expected to contribute to the magnetic interference experienced by the onboard magnetometers. The engine was tested in both the static (not running) and powered (running) states. When testing in the powered state, it was necessary to determine the effect of varying engine speed on magnetic field at the wingtip magnetometers. The frequency of the magnetic field effect was determined to evaluate design criteria pertaining to time-varying sources in the 0 to $5 \mathrm{~Hz}$ bandwidth.

A test procedure similar to the rotation testing described in Section 4.2.2 was conducted for the engine assembly. The results of this test yielded inconclusive results, with low repeatability between multiple data sets. The results for the rotational testing investigation are outlined in [29]. It was found that using the gradiometer test procedure described in Section 4.2 .3 yielded very repeatable results.

\subsubsection{Gradiometer Test}

A custom fuselage cradle was designed and built to allow for easy translation of the engine assembly and of the full-scale GeoSurv II prototype during gradiometer testing. The fuselage cradle was tested to ensure that it did not affect results. The engine was mounted to the fuselage, which was then attached to the fuselage cradle. For engine gradiometer testing, all magnetic components within the fuselage not part of the engine assembly were removed. A test track was defined, and the effect of the engine was measured at varying distances from sensors arranged in a gradiometer configuration. Figure 4.16 shows the setup used during experimentation. 


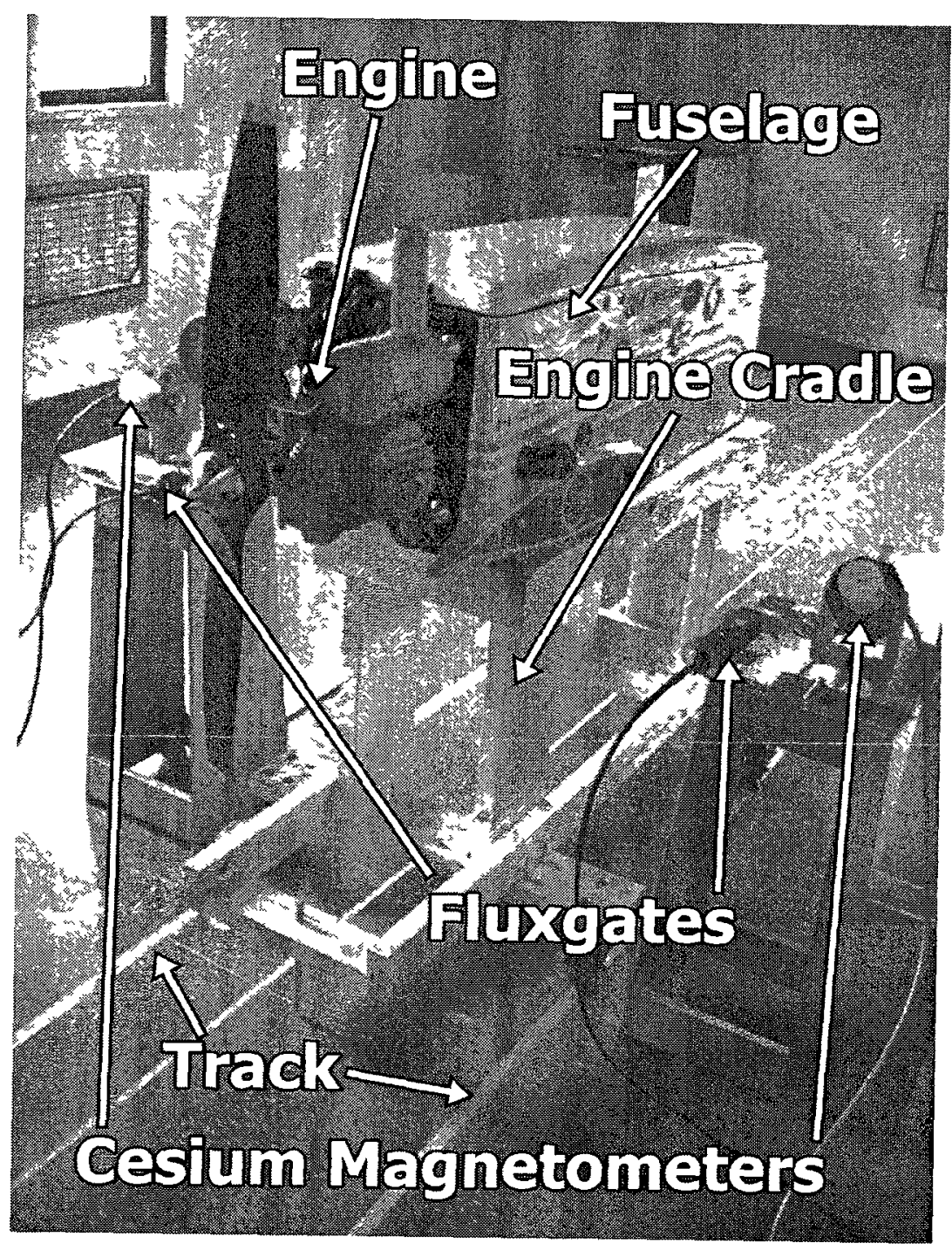

Figure 4.16: Experimental setup used during engine gradiometer testing Engine assembly was translated at incremental distances along a predefined track and measurements were taken of the inagnetic field effect at each location

This test was conducted in two cases with the fuselage translating in the longitudinal (tail-nose) direction and in the lateral (port-starboard) direction. The reference track was adjusted accordingly. This testing was completed without reference sensors, so the offset values were calculated using (4.1), and the corrected values were calculated using (42) only. All distances are referenced from the location of the centre of the intake manifold whereby positive distances are in the forward direction and 
negative distances are in the aft direction of the fuselage. From Figures 4.17 and 4.18 , it can be seen that the experimental data is suitable for characterization strategies to localize any sources of magnetic noise within the engine assembly. The peak magnetic noise occurs slightly forward of the intake manifold.
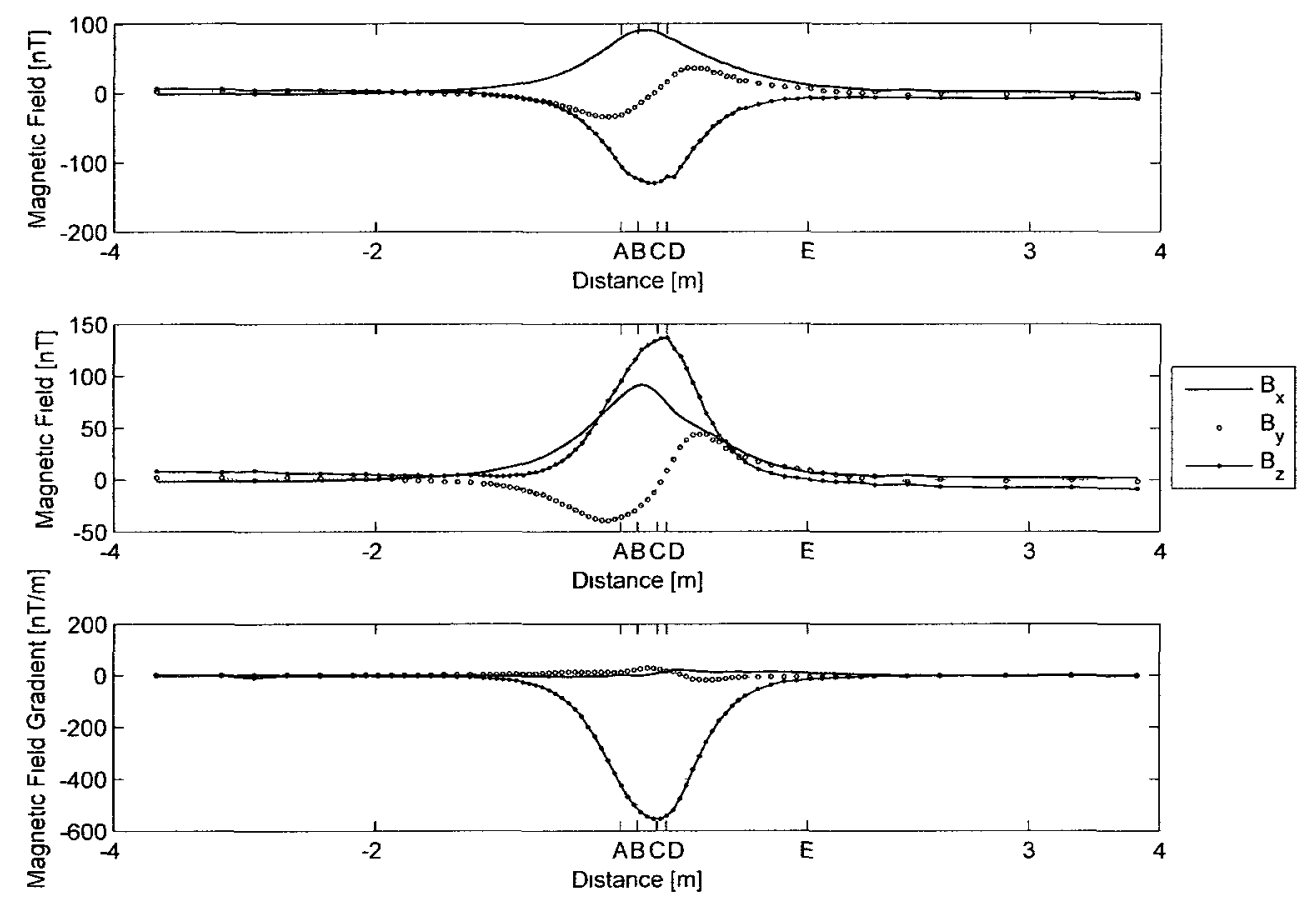

Figure 4.17: Behaviour of magnetic field for the GeoSurv II fuselage/engine moving past two fluxgates in a gradiometer configuration each located $0.39 \mathrm{~m}$ from the fuselage centreline. The starboard magnetometer (top), port magnetometer (middle), and magnetic field gradient (bottom) are shown. Reference points: (A) rear of propeller hub, (B) cylinder centre, (C) front of engine block, (D) front of carburetor, and (E) nose. The peak magnetic noise occurs slightly forward of the intake manifold. 

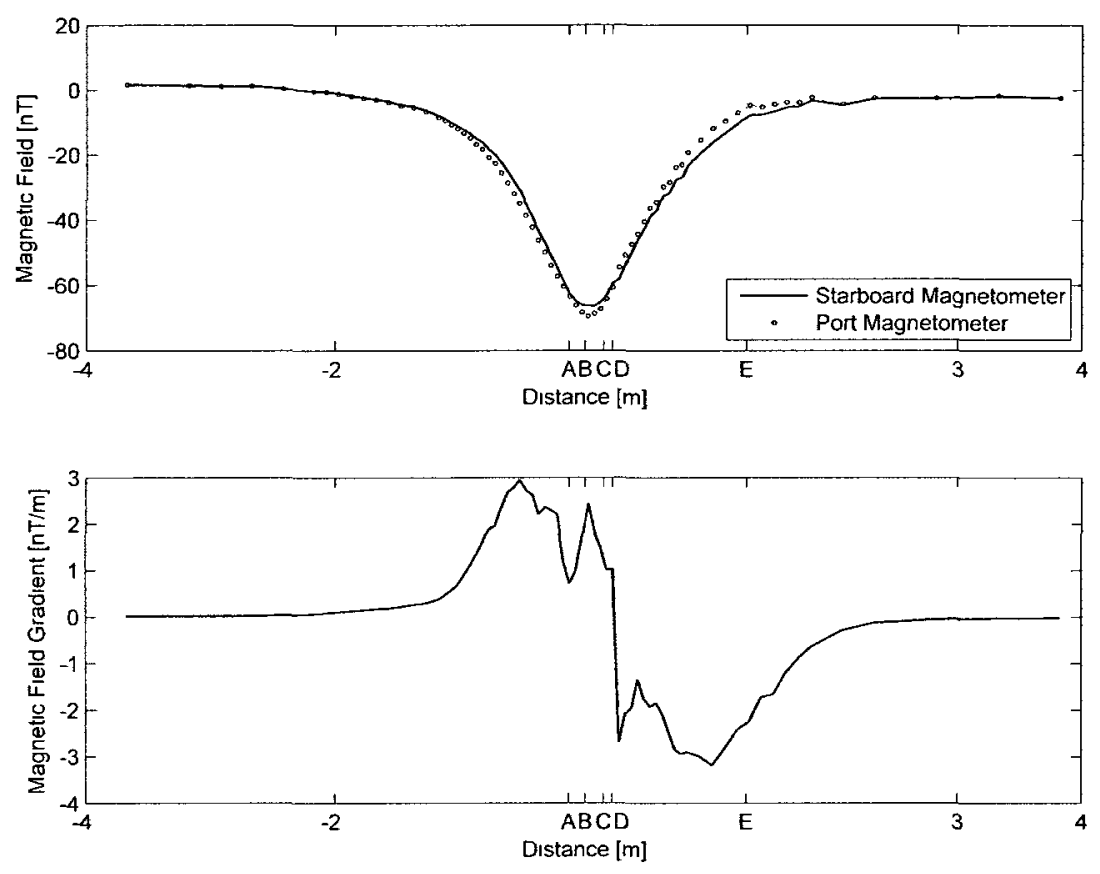

Figure 4.18: Behaviour of magnetic field for the GeoSurv II fuselage/engine moving past two Cesium magnetometers in a gradiometer configuration each located $0.55 \mathrm{~m}$ from the fuselage centreline. The top shows the magnetic field effect at the starboard and port magnetometers, respectively, and the bottom plot shows the gradient between magnetometers. Axis reference labels: (A) rear of propeller hub, (B) cylinder centre, (C) front of engine block, (D) front of carburetor, and (E) nose. The peakiness of the magnetic field gradient plot is due to the small differences in the total field values, which is further exasperated by small variations in fuselage/engine heading between measurement points.

Testing was also conducted to determine the behaviour of rotating the engine at each distance step. A transient magnetic noise effect from engine rotation was expected due to the movement of ferromagnetic components such as the crankshaft and connecting rods, as well as the ignition timing magnets located on the propeller hub which are of roughly equal and opposite polarity. Due to the presence of these components, the transient effect of the engine was expected to occur at the operating 
frequency of the engine. A similar procedure was followed with larger distance increments for the length of the track. The propeller was rotated by hand at approximately 30 RPM at each measurement location. For this test, the peak-to-peak difference in the magnetic field was calculated for each distance to determine the effect of running the engine. The effect of simulating the running engine at each distance, $B_{d, p r o p}$, for each distance, $d$, can be calculated at each sensor using the following:

$$
\vec{B}_{d . p r o p}=\vec{B}_{d, \max }-\vec{B}_{d, \min }
$$

where $\vec{B}_{d, \max }$ and $\vec{B}_{d, \min }$ are the maximum and minimum magnetic field values over the sample period. A typical transient profile of the magnetic noise at each sensor is shown in Figure 4.19 for the measurement at $0.0254 \mathrm{~m}$. 

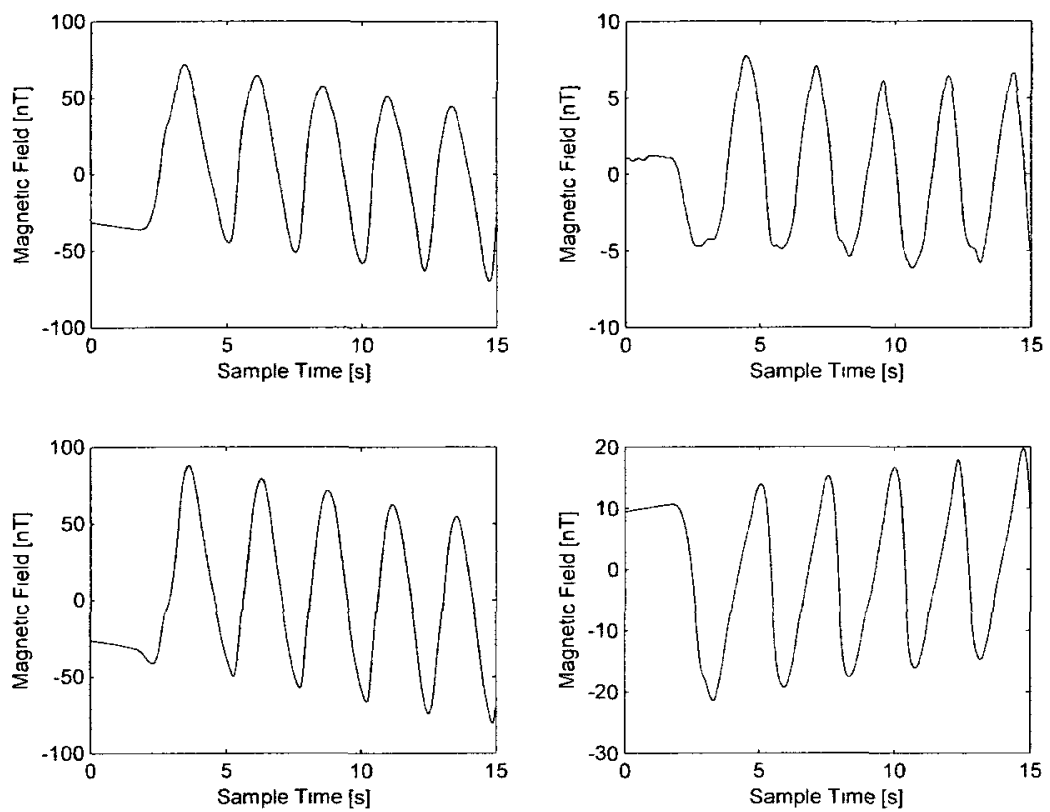

Figure 4.19: Transient magnetic noise profile for each sensor for the GeoSurv II fuselage/engine moving past two fluxgate magnetometers in a gradiometer configuration at the $0.0254 \mathrm{~m}$ step along the motion track while manually rotating the propeller. The fluxgate and Cesium magnetometers were located at $0.39 \mathrm{~m}$ and $0.55 \mathrm{~m}$, respectively, from the fuselage centreline. The transient signature is displayed for the fluxgate $B_{x}$ sensor (top left), $B_{y}$ sensor (top right), $B_{z}$ sensor (bottom left), and for the Cesium magnetometer sensor (bottom right).

Figures 4.20 and 4.21 display the magnetic field variation of manually cycling the engine with respect to distance from magnetometers. This gives an indication of the magnetic noise effect of a powered engine with respect to distance from the magnetometers. 

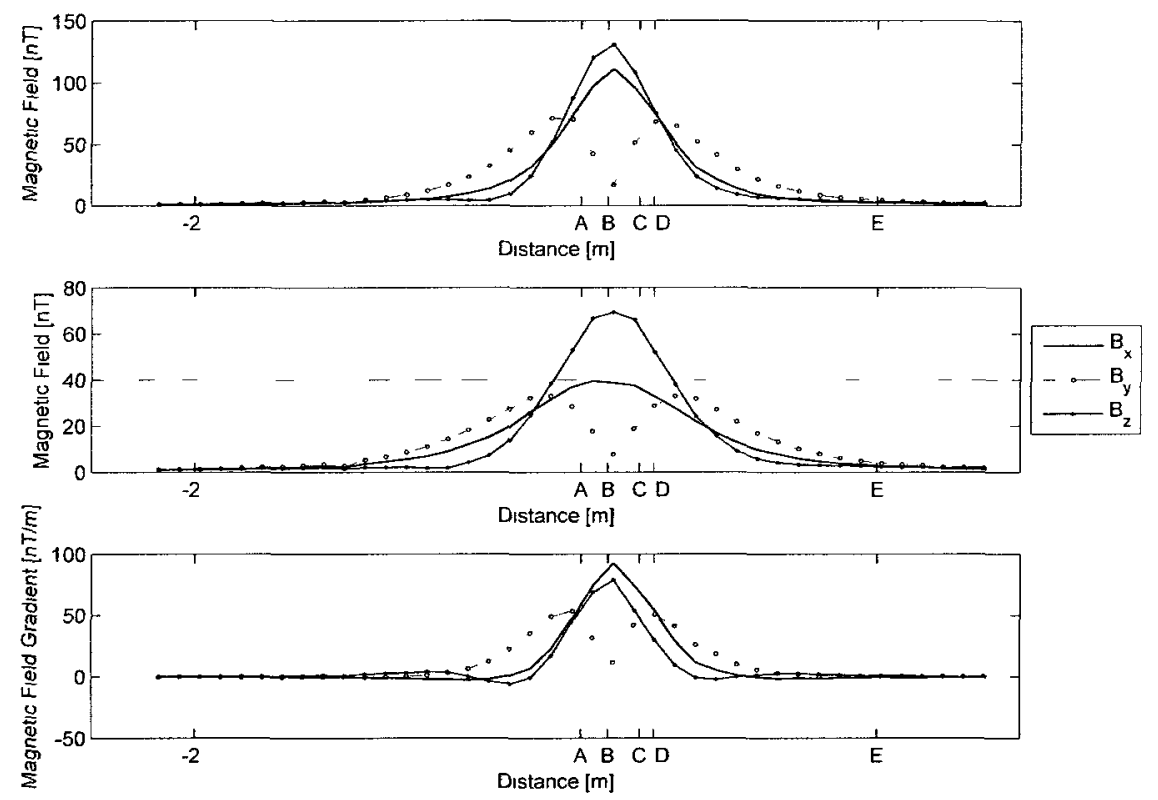

Figure 4.20: Amplitude of the transient magnetic field for the GeoSurv II fuselage/engine moving past two fluxgate magnetometers in a gradiometer configuration each located $0.39 \mathrm{~m}$ from the fuselage centreline while manually rotating the propeller. The transient amplitude is displayed for the starboard fluxgate (top), port fluxgate (middle), and bottom shows the inagnetic field gradient between the two fluxgates. Axis reference labels: (A) rear of propeller hub, (B) cylinder centre, (C) front of engine block, (D) front of carburetor, and (E) nose. Transient magnetic noise peaks slightly forward of the intake manifold. 

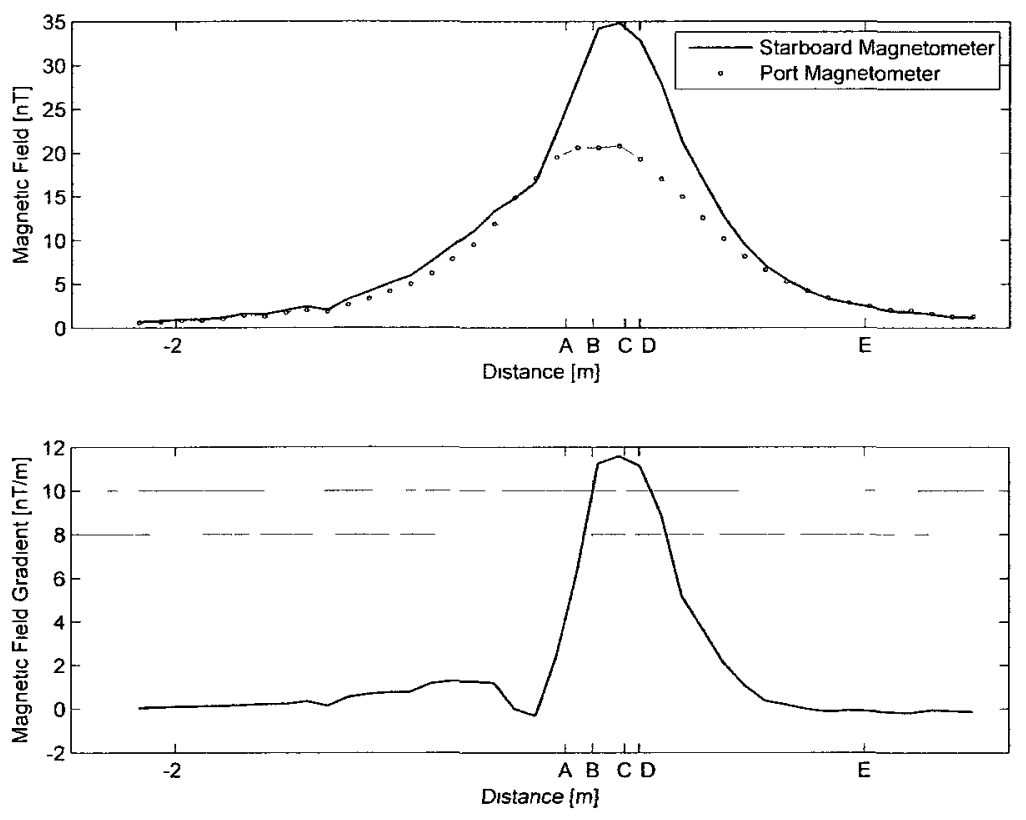

Figure 4.21: Amplitude of the transient magnetic field for the GeoSurv II fuselage/engine moving past two Cesium magnetometers in a gradiometer configuration each located $0.55 \mathrm{~m}$ from the fuselage centreline while manually rotating the propeller. The transient amplitude is displayed for the starboard and port magnetometers (top), and bottom shows the magnetic field gradient between the two magnetometers. Axis reference labels: (A) rear of propeller hub, (B) cylinder centre, (C) front of engine block, (D) front of carburetor, and (E) nose. Transient magnetic noise peaks slightly forward of the intake manifold, which also corresponds to the location of the largest magnetic field gradient. 


\subsubsection{Powered Engine Testing}

A powered engme test was conducted on a pathway at the Geomagnetic Laboratory to determine if there were any engine components that produced time-varying magnetic fields in the 0 to $5 \mathrm{~Hz}$ range. The test procedure involved mounting a Cesium magnetometer at each wingtip location. and the utilization of a reference magnetometer. The wings and empennage where not included in the assembly for this test, as is shown in Figure 4.22.

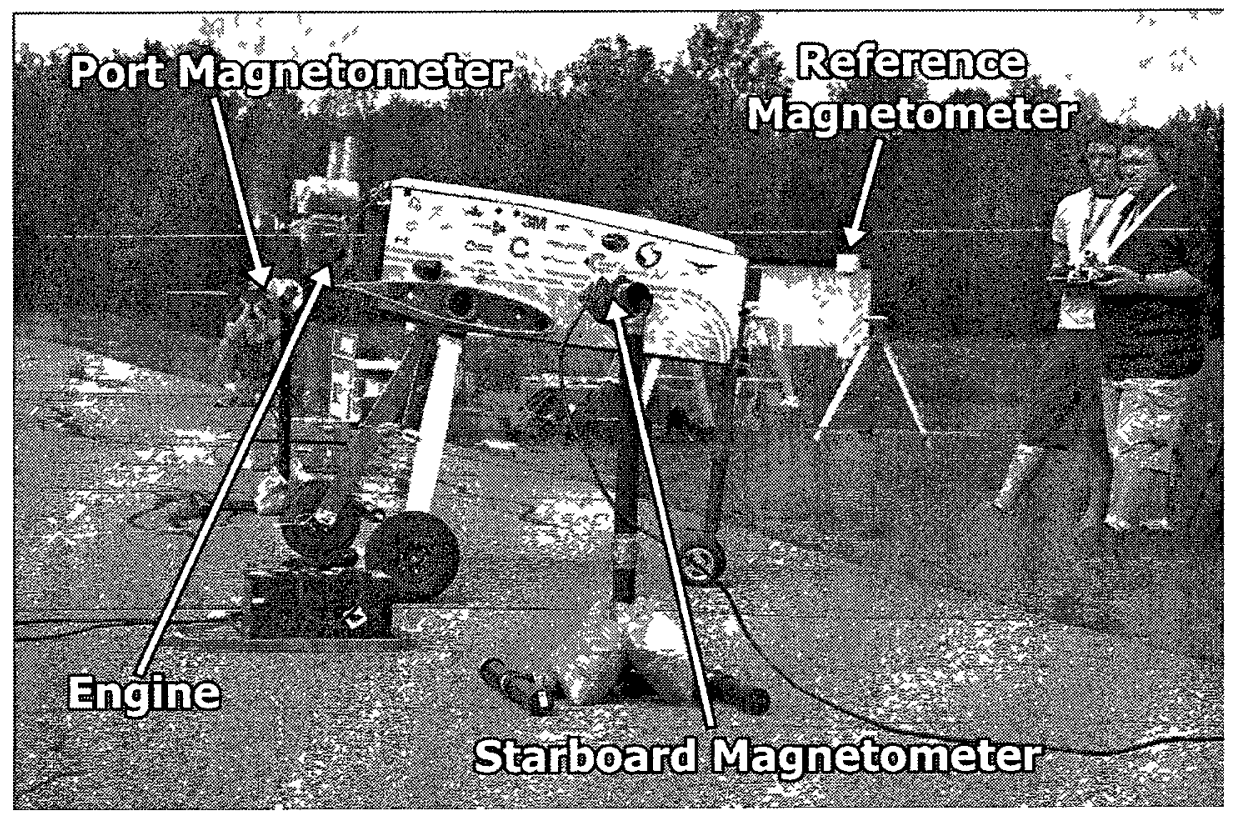

Figure 4.22: Experimental setup used for the engine run. Starboard and port magnetometers were positioned in locations corresponding to where they would be mounted if the UAS were fully assembled. The reference magnetometer was sufficiently distant to be unaffected by any engine runs or preparation activity.

The engine was operated at multiple speed settings for approximately 20 seconds each. The engine speeds tested were $2140,3140,3160,3975$, and 4150 RPM. The mean value was removed from each data set, and the data was analyzed in the frequency domain. MATLAB's Fast Fourier Transform (FFT) and Power Spectral Density 
(PSD) functions were utilized for this analysis.

Prior to collecting magnetic noise data during a powered engine run, the effect of rotating the engine manually was analyzed for magnetometers placed at wingtip locations during full scale testing. From Figure 4.23 , it can be observed that the peak-to-peak amplitudes of the transient magnetic field are $0.6 \mathrm{nT}$ and $0.4 \mathrm{nT}$ for the starboard and port magnetometers, respectively. For this reason, it was expected that these transient magnetic field amplitudes would be present at the engine operating frequency during a powered engine run. These results are lower than those measured in Figure 4.21 due to the increased magnetometer spacing.
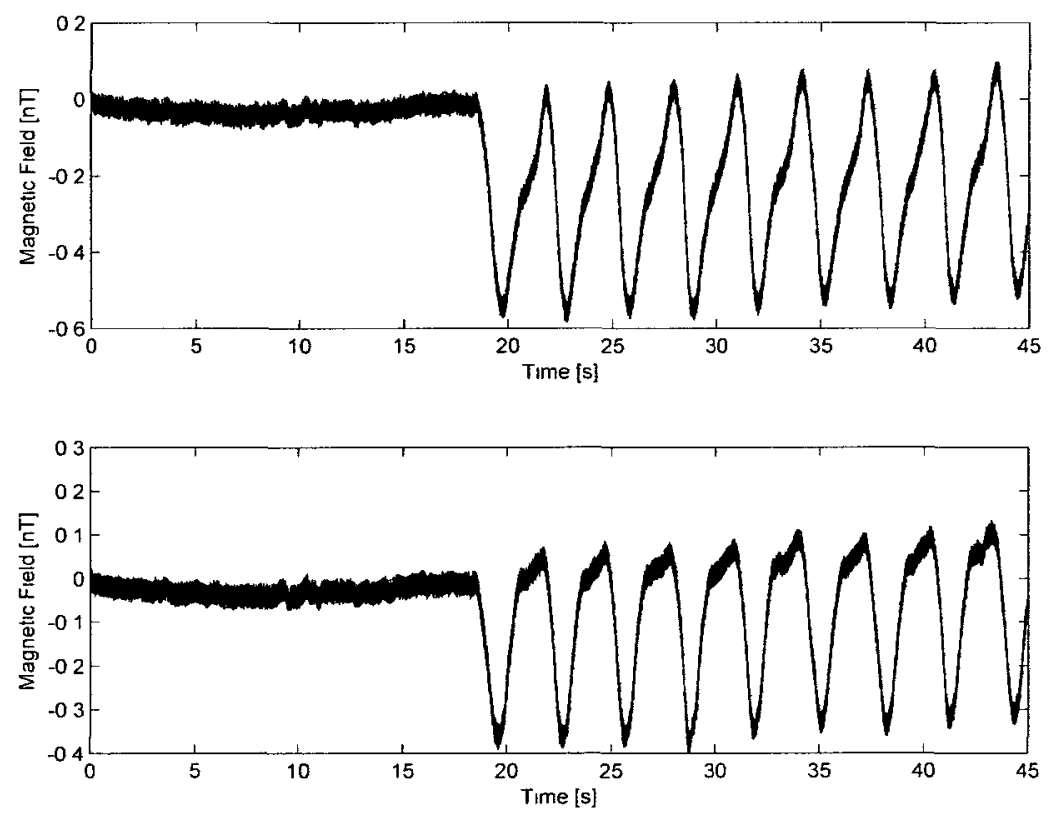

Figure 4.23: Magnetic field behaviour while manually rotating propeller to simulate a running engine with magnetometers at wingtip spacing. Shown are the starboard (top) and port (bottom) magnetometers. Amplitudes of $0.6 \mathrm{nT}$ and $0.4 \mathrm{nT}$ were observed for the transient magnetic field at the starboard and port wingtips, respectively. 
Initial analysis was conducted using MATLAB's Fast Fourier Transform (FFT), the results of which can be seen in Figures 4.24 and 4.25 which show the same data but with different scaling on the frequency axis. From Figure 4.24, it can be observed that the dominant magnetic field frequency was $60 \mathrm{~Hz}$, which corresponds to the switching frequency of the utility grid. There was also some aliasing visible at the $20 \mathrm{~Hz}$ frequency, due to the sampling frequency of $160 \mathrm{~Hz}$. Importantly, there was no discernible magnetic field effect at the engine operating frequency. Additionally, because of the 0 to $5 \mathrm{~Hz}$ bandwidth requirement on magnetic noise, this bandwidth was studied more closely as seen in Figure 4.25 which shows that the transient engine noise experienced appears to meet the design specifications. The low frequency $(\leq 0.25$ $\mathrm{Hz}$ ) noise seen in Figure 4.25 is present at the reference magnetometer as well, which led to the conclusion that this effect was likely due to aliasing produced by the FFT rather than onboard sources. 

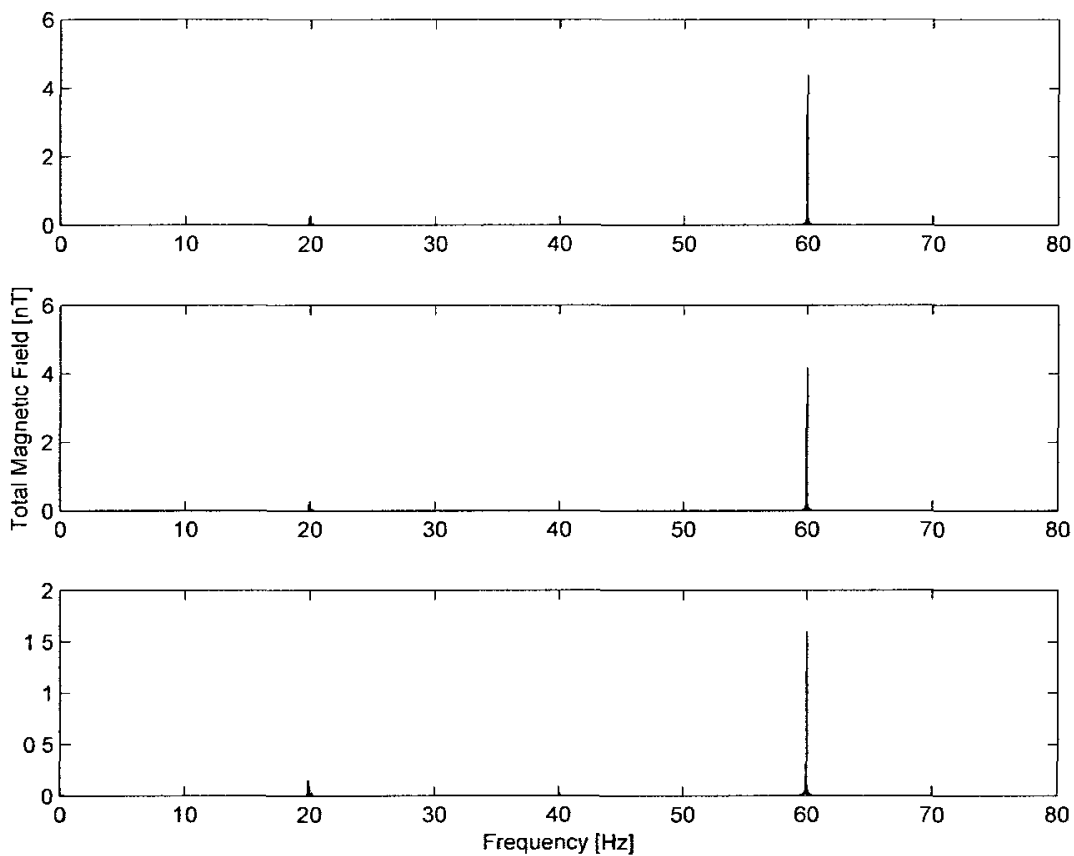

Figure 4.24: Magnetic noise versus frequency for the GeoSurv II engine operating at an average speed of $2140 \mathrm{RPM}(35.7 \mathrm{~Hz})$. Shown are the starboard wingtip magnetometer (top), port wingtip magnetometer (middle), and reference magnetometer (bottom). The noise is largest at the $60 \mathrm{~Hz}$ frequency, corresponding to the utility grid switching frequency. 

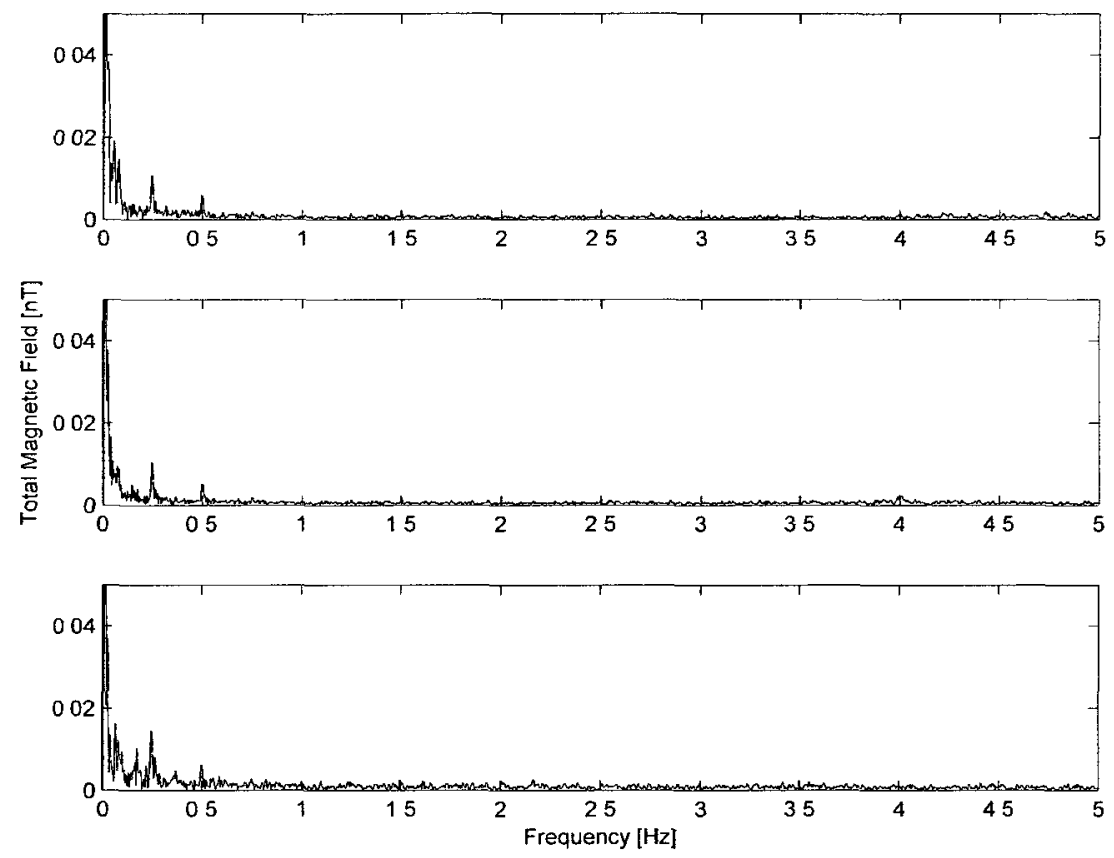

Figure 4.25: Magnetic noise versus frequency for the GeoSurv II engine operating at an average speed of 2140 RPM $(35.7 \mathrm{~Hz})$. Shown are the starboard wingtip magnetometer (top), port wingtip magnetometer (middle), and reference magnetometer (bottom). The low frequency $(\leq 0.25 \mathrm{~Hz})$ noise is present at all magnetometers. leading to the conclusion that this effect was likely due to aliasing produced by the FFT rather than onboard sources since nothing onboard is known to operate that slowly. 
The results of the PSD analysis can be seen in Figure 4.26, where it can be noted that a magnetic field effect appears at the engine operating frequency. Additionally, there is a transient source present at both wingtip magnetometers but not at the reference magnetometer, which is operating at a frequency of approximately $13 \mathrm{~Hz}$. This frequency is above the 0 to $5 \mathrm{~Hz}$ design requirement but is still of interest since it does not appear to be dependent upon engine speed for all engine speeds analyzed. This analysis was done for a variety of engine speeds which allows for the alias frequencies of each operating point to be tracked to determine if they pose a concern. From the PSD analysis, it appears that the transient magnetic signature produced by the powered engine will not be a problem with regards to magnetic signature control.
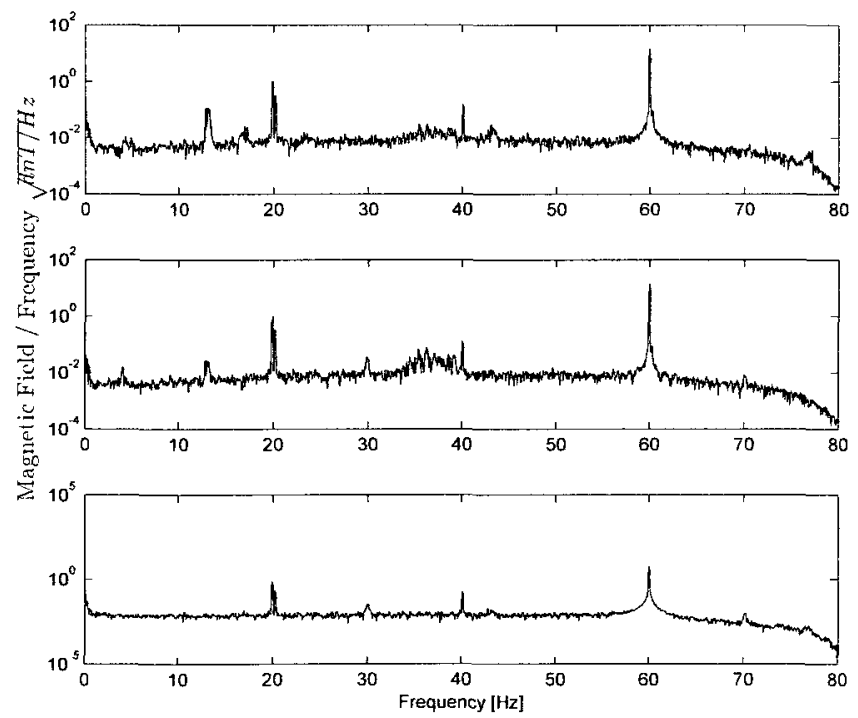

Figure 4.26: Power spectral density analysis of magnetic noise for the GeoSurv II engine operating at an average speed of 2140RPM $(35.7 \mathrm{~Hz})$. Shown are the starboard (top), port (middle), and reference (bottom) magnetometers. Low amplitude magnetic noise is observed at the engine operating speed. As well, there is a magnetic noise source operating at $13 \mathrm{~Hz}$ which is present at the wingtip sensors, but not at the reference sensor. The $20 \mathrm{~Hz}$ noise appears at all engine frequencies. 


\subsection{Avionics Testing}

The gradiometer test procedure of Section 4.2 .3 was also applied to study the magnetic signature of the flight avionics. The flight avionics were removed from the fuselage and mounted on a nonmagnetic tray to allow for easy translation in both the longitudinal and lateral directions. The flight avionics rack was powered using three A123 racing batteries which were also included on the avionics tray. The experimental setup used is shown in Figure 4.27.

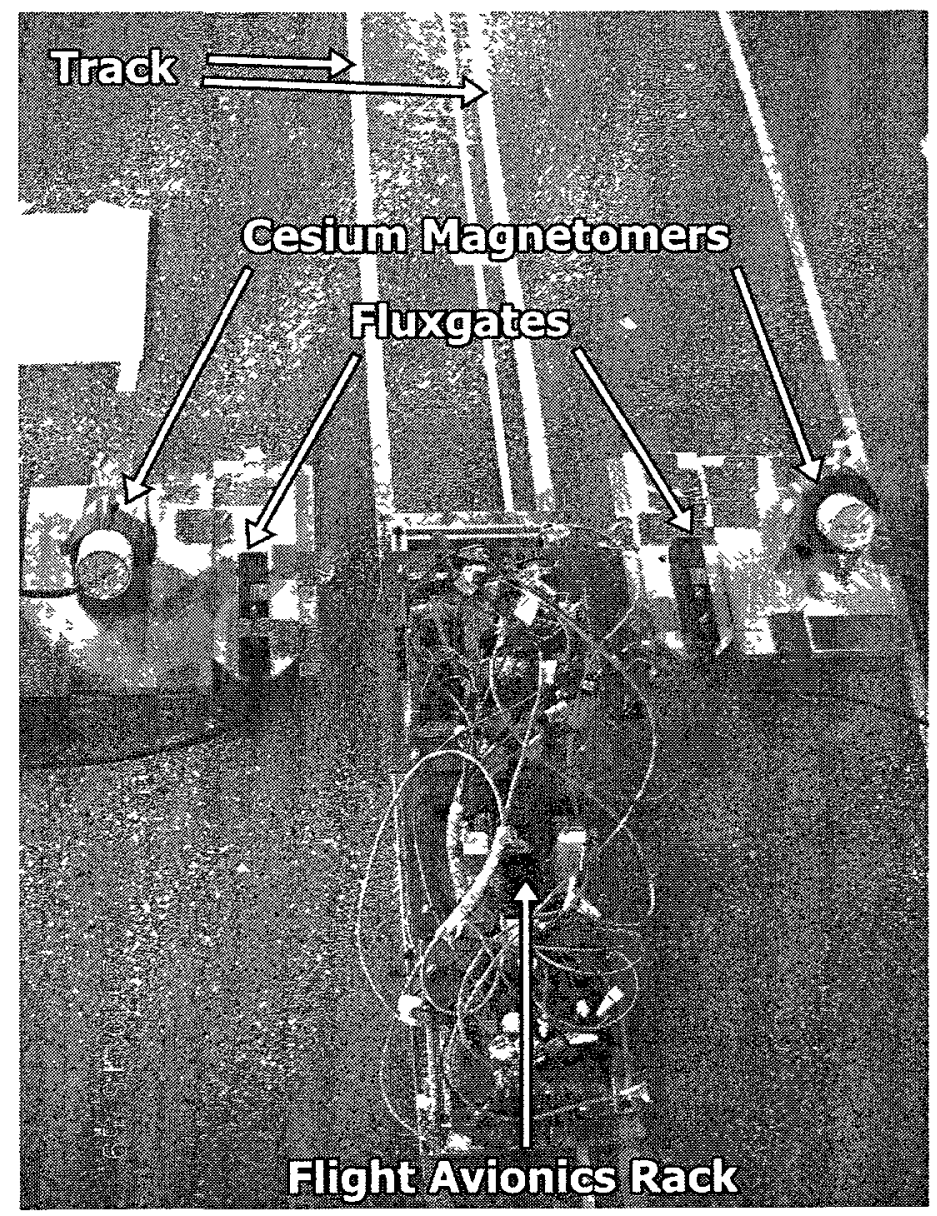

Figure 4.27: Experimental setup used during flight avionics gradiometer testing. Flight avionics package was translated at incremental distances along a predefined track and measurements were taken of the magnetic field effect at each location. Shown for the longitudinal direction here. 
All distances are referenced from the centre point of the leading edge of the rack, with the forward direction being positive along the longitudinal axis and the starboard direction being positive along the lateral axis. This test was conducted in two cases with the avionics platform translating in the longitudinal (tail-nose) direction and in the lateral (port-starboard) direction, with reference track was adjusted accordingly for each direction. Both tests were conducted along a south-north track. This testing was completed without reference sensors, so the offset values were calculated using (4.1), and the corrected values were calculated using (4.2) only.

Figure 4.28 indicates that there are multiple magnetic field sources along the length of the flight avionics rack as indicated by the multiple peaks and troughs. The difference in total magnetic field between the two magnetometers during the longitudinal gradiometer test, as seen in Figure 4.29, is small compared to the difference in field components at each fluxgate, seen in Figure 4.28. This is due to the increased sensor separation for the Cesium magnetometers as compared to the fluxgates. 

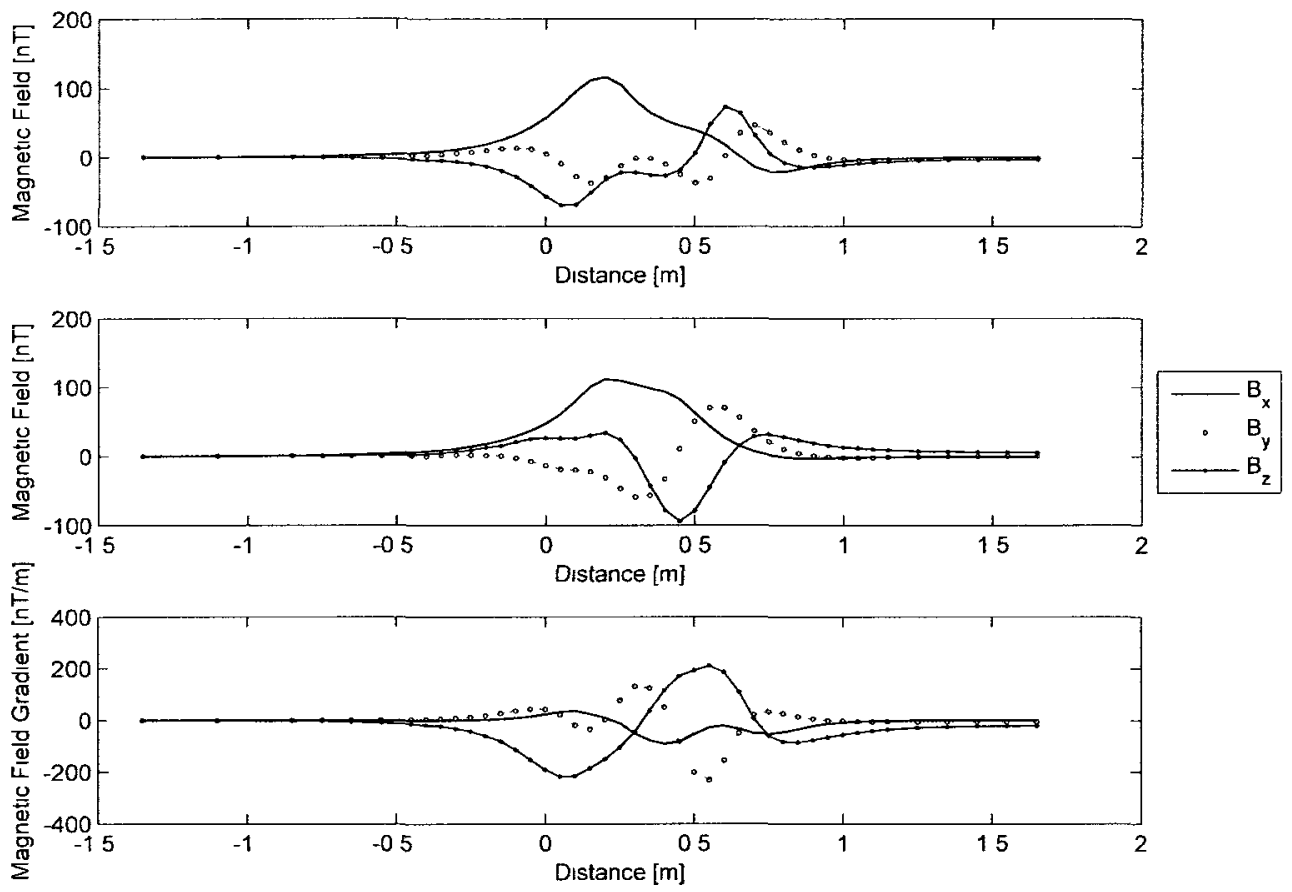

Figure 4.28: Behaviour of magnetic field for the flight avionics moving longitudinally between two fluxgate magnetometers in a gradiometer configuration each located $0.22 \mathrm{~m}$ from the avionics tray centreline. Measurements at the starboard fluxgate (top), port fluxgate (middle), and the magnetic field gradient between the two fluxgates (bottom) are shown. Multiple sources of magnetic noise are present along the length of the flight avionics rack. 

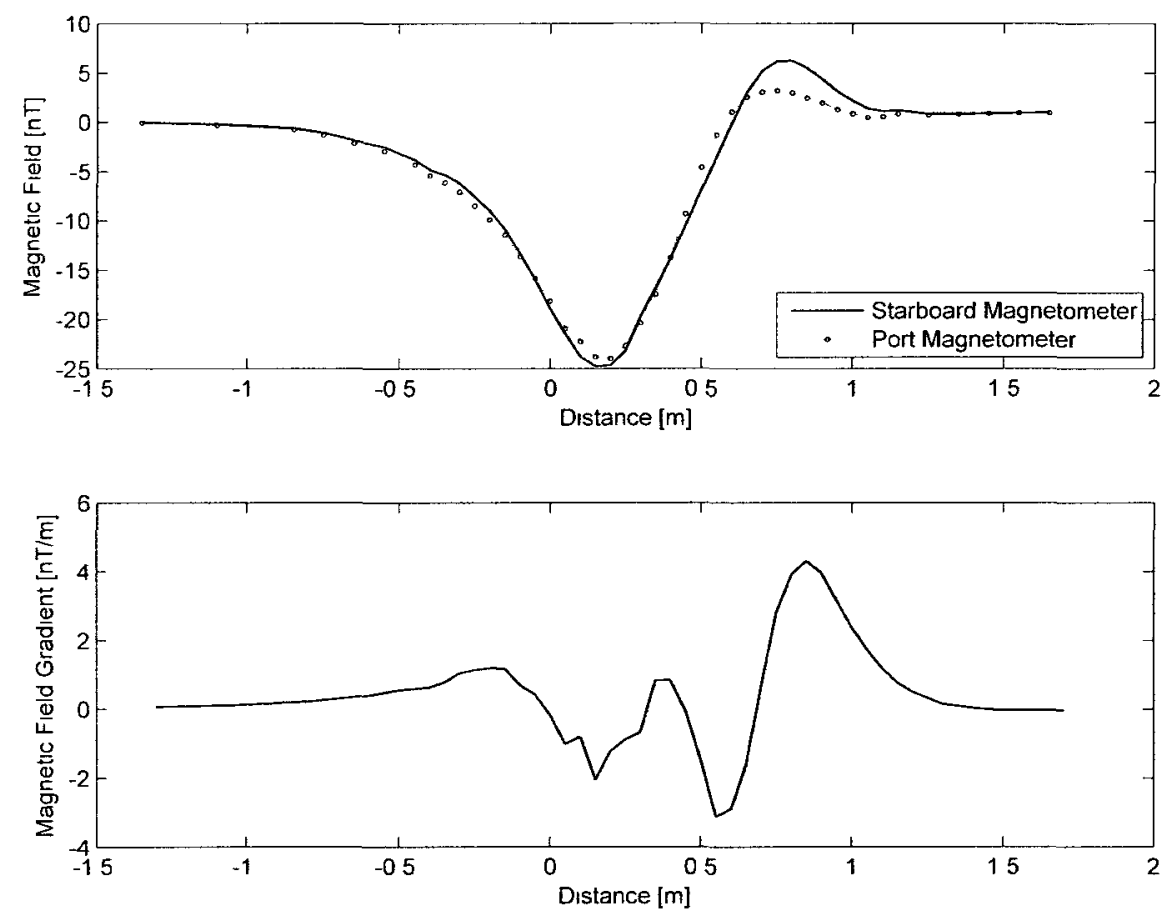

Figure 4.29: Behaviour of magnetic field for the flight avionics moving longitudinally between two Cesium magnetometers in a gradiometer configuration each locat ed $0.38 \mathrm{~m}$ from the avionics tray centreline. The readings at both magnetometers (top), as well as the magnetic field gradient between magnetometers (bottom) are shown. The magnitude of the magnetic field effect is quite small relative to the servos and engine assembly. 
The results from the lateral gradiometer test indicate that all sources are $\approx 0.1 \mathrm{~m}$ port of the avionics tray centreline. This can be seen by the location of the peaks and troughs in Figures 4.30 and 4.31. Additionally. using (4.7) the magnetic noise effect of the flight arionics was concluded to be negligible due to its minute magnetic far-field effect at distances similar to the avionics-magnetometer distances on GeoSurv II.
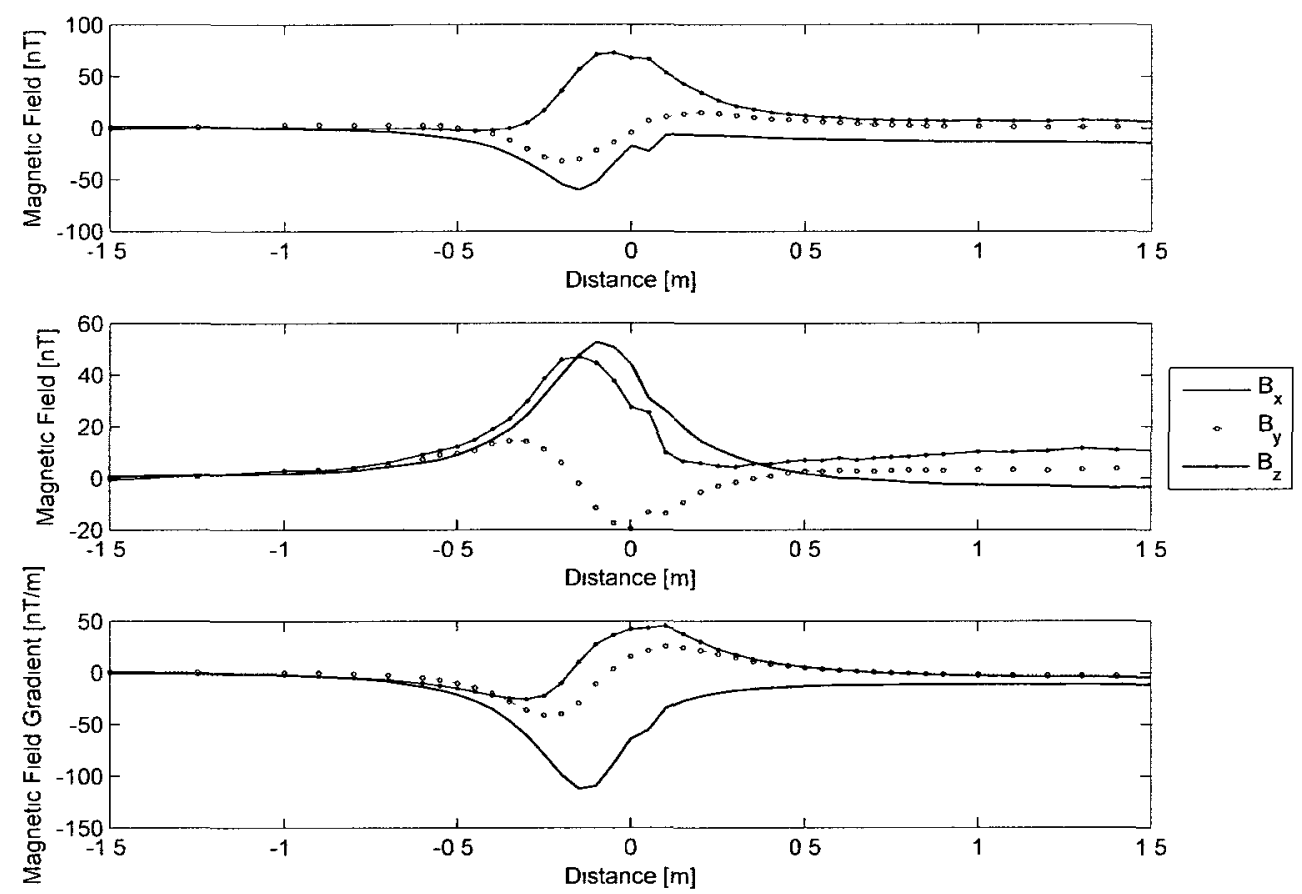

Figure 4.30: Behaviour of magnetic field for the flight avionics moving laterally between two fluxgate magnetometers in a gradiometer configuration each located $0.48 \mathrm{~m}$ from the avionics tray centreline. Measurements at the starboard fluxgate (top), port fluxgate (middle), and the magnetic field gradient between the two fluxgates (bottom) are shown. Magnetic noise sources appear to be located $\approx 0.1 \mathrm{~m}$ port of the avionics tray centreline. 

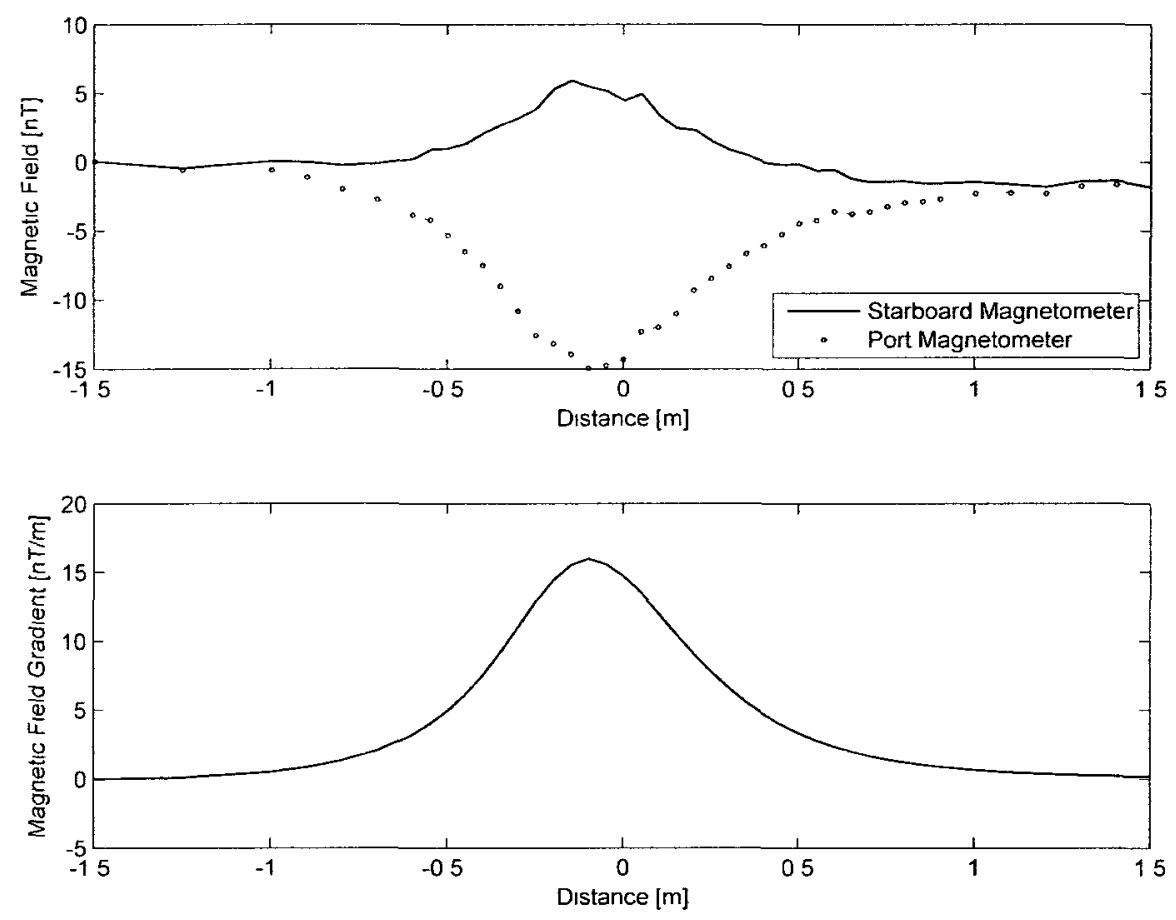

Figure 4.31: Behaviour of magnetic field for the flight avionics moving laterally between two Cesium magnetometers in a gradiometer configuration each located $0.64 \mathrm{~m}$ from the avionics tray centreline. Readings at both magnetometers (top), as well as the magnetic field gradient between magnetometers (bottom) are shown. Magnetic noise sources appear to be located $\approx 0.1 \mathrm{~m}$ port of the avionics tray centreline.

\subsection{Component Testing Conclusions}

The results of the investigation into the magnetic signature of individual GeoSurv II prototype components concluded that the servo actuators were the largest contributors of magnetic noise, followed by the engine assembly. Since the engine assembly constitutes a magnetic noise source that is not easily modelled, attention must be given to reducing the magnetic signature of the engine through the replacement of ferromagnetic parts with nonmagnetic equivalents; this may even require the development of an engine composed of completely nonmagnetic components. Servos, 
conversely, are easily represented by simple magnetic dipole models, therefore the development of servo models is crucial for implementing proper optimization and cancellation schemes. Since the positioning and orientation of each servo is flexible in the GeoSurv II design, focus was put primarily on these components for minimizing the magnetic signature of the UAS. 


\section{Chapter 5}

\section{Characterization Strategies}

\subsection{Introduction}

Magnetic field sources must be characterized for inclusion into models and optimization algorithms. As was introduced in Section 2.5.1, the magnetic source characterization strategies developed in this thesis will consist of representative magnetic point dipoles. Sources may be modelled by one or more magnetic point dipoles, each characterized by six parameters specifying the location, magnitude, and orientation: $\vec{m}_{x}, \vec{m}_{y}, \vec{m}_{z}, x, y, z$. The objective of characterization strategies is to determine the six parameter values such that the error is minimized between experimental magnetic field measurements and magnetic ficld values calculated using equivalent point dipole models.

\subsection{Requirement for an Improved Characteriza- tion Scheme}

The MoDEM model previously developed by Wells [1], modelled sources using two magnetic point dipoles as outlined in Section 2.5.1. The objective function used in 
the MoDEM strategy was designed to minimize the error between the total field from the data collected experimentally and the total field modelled by point dipoles:

$$
\text { Error }=\frac{1}{N} \sum_{\imath=1}^{N}\left(\left\|\vec{B}_{\imath, \text { experımental }}\right\|-\left\|\vec{B}_{\imath, M o D E M}\right\|\right)^{2},
$$

where $N$ is the number of points fitted, and $\vec{B}_{\text {expermental }}$ and $\vec{B}_{M o D E M}$ are the experimentally measured and the modelled magnetic flux density, respectively.

The MoDEM objective function was selected to give equal weighting to fitting the $\vec{B}_{x}, \vec{B}_{y}$, and $\vec{B}_{z}$ curves, but instead of giving equal weighting to the vector components, the MoDEM objective function considers only the total field values. It was found that the objcctive function defined in MoDEM provided an excellent fit betwcen the experimental and modelled total field curves, but had a tendency to less accurately fit the individual $\vec{B}_{x}, \vec{B}_{y}$, and $\vec{B}_{z}$ components. Although the MoDEM strategy is capable of accurately capturing the vector components, it is susceptible to ambiguity about the proportion to which each vector component contributes to the total field value. This ambiguity is highlighted in Figure 5.1. 


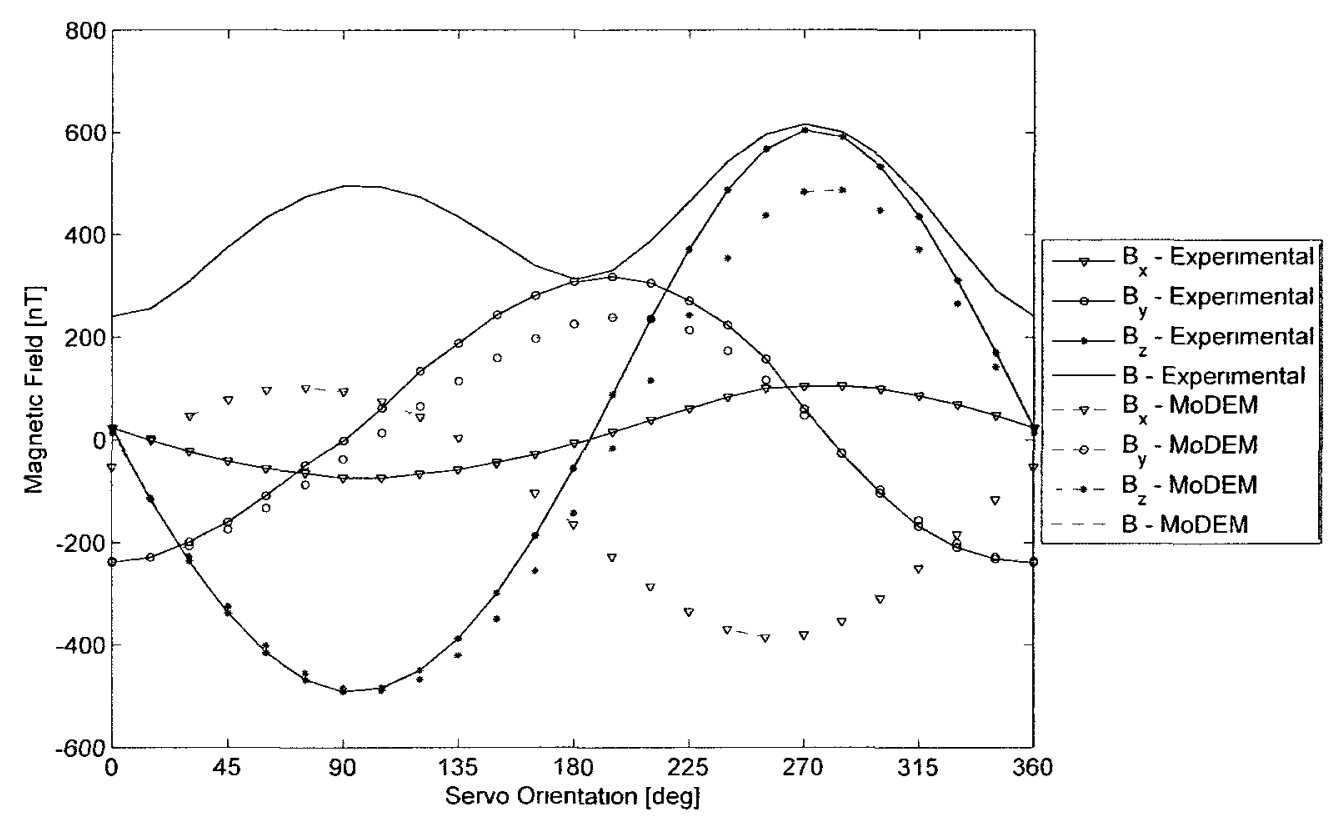

Figure 5.1: MoDEM characterization scheme with fitness function specified by Wells [1] for a single Hitec HSR-5990TG servo. Measurements taken at a distance of $0.30 \mathrm{~m}$, with rotation about the x-axis of the servo. Excellent fit between the experimental and modelled total ficld curves, but a less accurate fit between the experimental and modelled vector component curves. The difference is most noticeable for the $\vec{B}_{x}$ field component.

Magnetic noise, as defined for the GeoSurv II application, is the deviation in the total magnetic field from the naturally occurring background field. Since the net field at any given point is the vector sum of the contributions. the total noise at a given measurement point is dependent on the vector contributions of both the background field and the magnetic noise sources, rather than the sum of the total field contributions. This point is illustrated in Figure 5.2 where it is shown that two interference vectors of the same magnitude but different orientation will result in two different magnetic noise levels. 
Magnetometer

A

Interference
Vector

Background

Field Vector

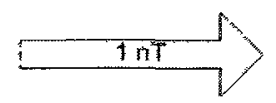

\section{Interference \\ $+$ \\ Background \\ Field}

\section{Magnetic \\ Noise}

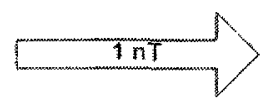

Magnetometer

B
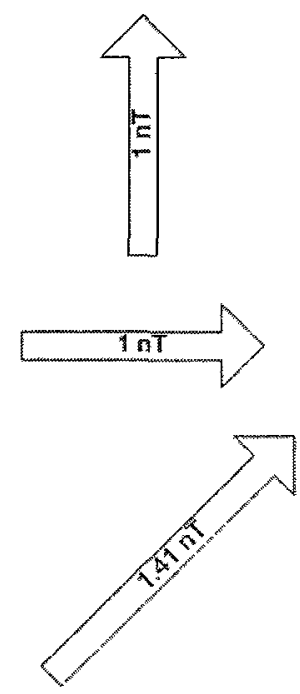

$0.41 \mathrm{nT}$

Figure 5.2: Effect of the interference vector interacting with the background field. Noise is measured as the total field deviation from the natural background field, meaning that knowledge of the vector contributions of each source is necessary.

Therefore, it was necessary to develop an alternative objective function to produce an improved fit between the experimental and modelled vector magnetic flux density values. A fitness function utilizing the error in the vector components was developed and implemented in an improved MoDEM model, known as MoDEM2. The new fitness function determines the mean square of the error in the vector components as follows: 


$$
\begin{aligned}
\text { Error }=\frac{1}{3}[ & \frac{1}{N} \sum_{\imath=1}^{N}\left(\vec{B}_{x . \imath . \text { experimental }}-\vec{B}_{x, \imath . M o D E M 2}\right)^{2} \\
& +\frac{1}{N} \sum_{i=1}^{N}\left(\vec{B}_{y . \imath \text { experimental }}-\vec{B}_{y, \imath . M o D E M 2}\right)^{2} \\
& \left.+\frac{1}{N} \sum_{\imath=1}^{N}\left(\vec{B}_{z . \imath . e x p e r i m e n t a l}-\vec{B}_{z, \imath . M o D E M 2}\right)^{2}\right]
\end{aligned}
$$

As shown in (5.2), the mean square error for all measurement points is calculated for the three vector components individually. The objective function is defined by the average of the vector mean square errors, as shown in (5.2). Consequently, by minimizing the error between the vector components, the error between the experimental and modelled total field components will also be minimized. The new MoDEM2 objective function consistently provides a better fit to the vector components while still closely matching the total field values. The result of the improved MoDEM2 objective function is shown in Figure 5.3. 


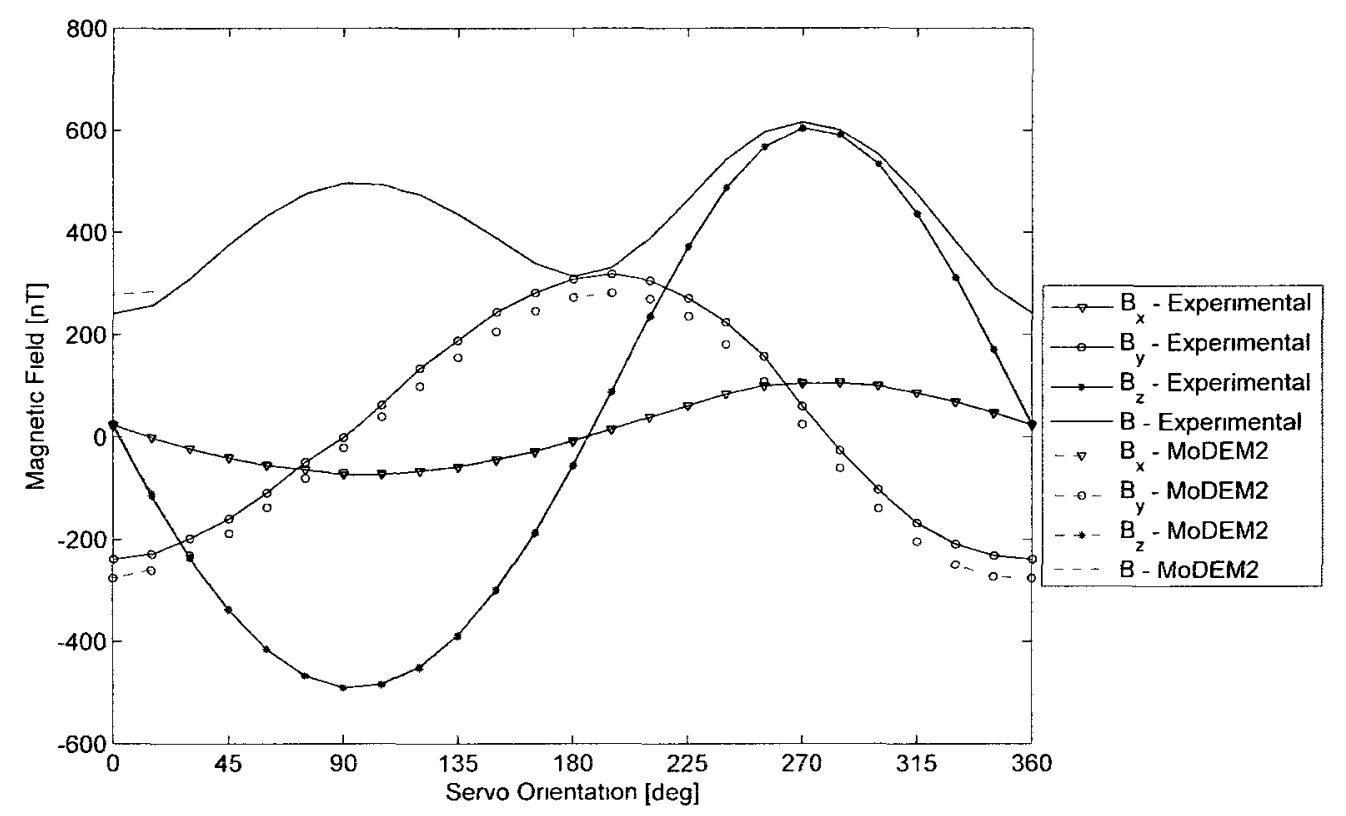

Figure 5.3: MoDEM2 characterization scheme with fitness function defined in this thesis for a single Hitec HSR-5990TG servo. Measurements taken at a distance of $0.30 \mathrm{~m}$, with rotation about the $\mathrm{x}$-axis of the servo. Good fit between the experimental and modelled vector and total field curves.

Additionally, if the position vector component parallel to the axis of rotation used during measurements is small, the variation of the magnetic field component parallel to the axis of rotation will be small compared to the other vector components. This causes the MoDEM and MoDEM2 models to have difficulty accurately characterizing the respective dipole component value, i.e. if rotating about the x-axis and $\vec{r}_{x}$ is small, then $\vec{B}_{x}$ will be a low amplitude sinusoid, such as in Figures 5.1 and 5.3. This results in a bias towards modelling the magnetic field within the measurement plane, meaning that any calculation of the magnetic field outside of the measurement plane may be inaccurate if using the MoDEM and MoDEM2 strategies. This effect is most noticeable when performing testing with rotations about two or more axis of the component, and is illustrated in Figures 5.4 and 5.5 which show the MoDEM and 
MoDEM2 models, respectively, compared to experimental data collected for rotations about the servos $\mathrm{x}^{-}, \mathrm{y}^{-}$, and z-axes. It should be noted that in Figures 5.4, 5.5, 5.7, and 5.10 , the difference in behaviour shown for rotation about the $\mathrm{x}$-axis for the Hitec HSR-5990TG servo, when comparing the top left and bottom right subfigures is due to the different position vectors utilized between measurement apparatus; the top left, top right, and bottom left data was collected using the MMTA-3D apparatus, whereas the bottom right data was collected using the MMTA-1D apparatus. 

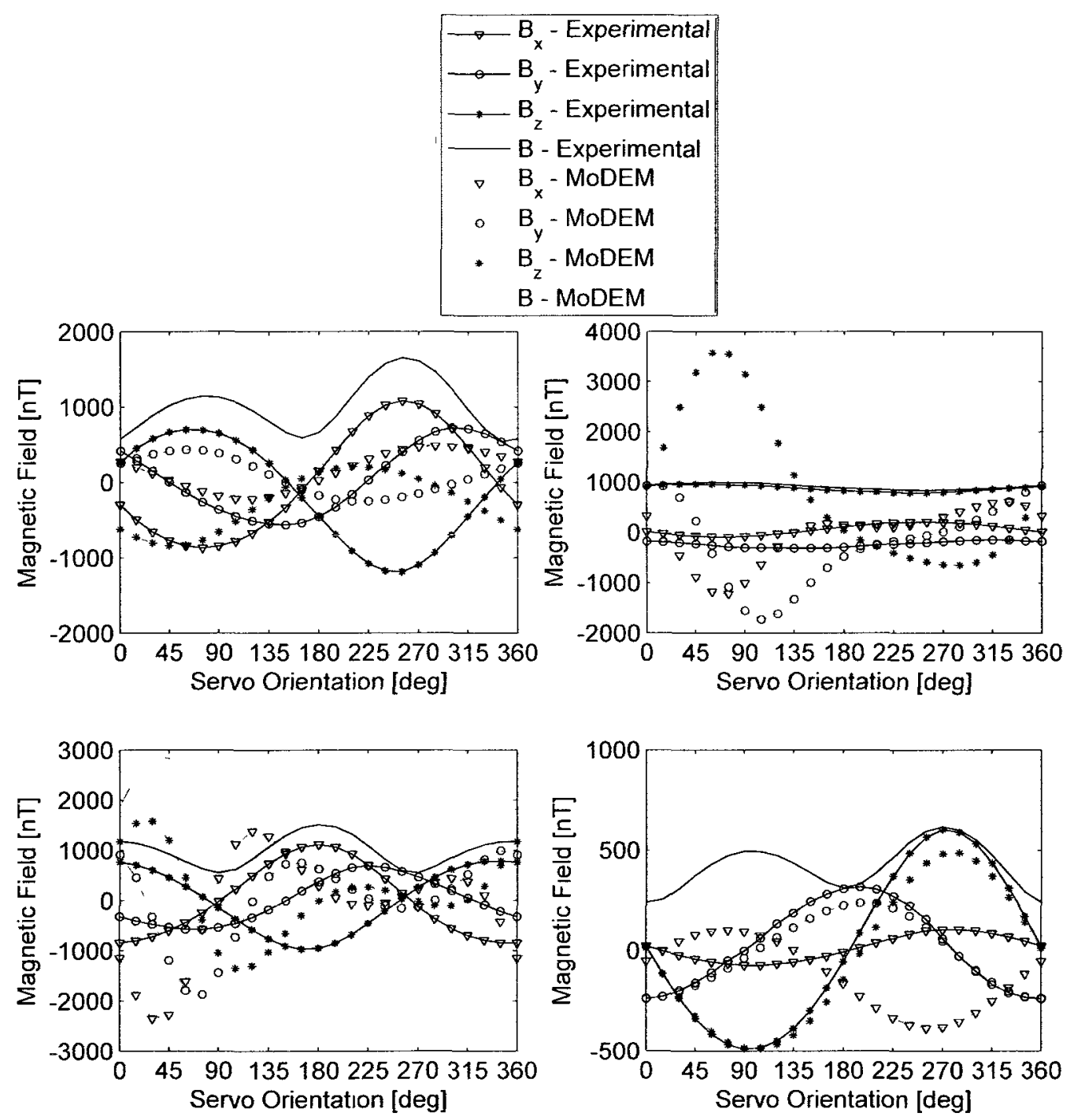

Figure 5.4: MoDEM characterization compared to experimental data collected in multiple measurement planes for a Hitec HSR-5990TG servo. Rotations about the $\mathrm{x}$-axis (top left), $\mathrm{y}$-axis (top right), and $\mathrm{z}$-axis (bottom left) are shown for measurements taken at $0.22 \mathrm{~m}$ from the servo, as well as the data used for initial characterization (bottom right). The MoDEM model does not necessarily characterize the servo accurately, leading to errors when measuring the vector and total field at points not used during the characterization. 


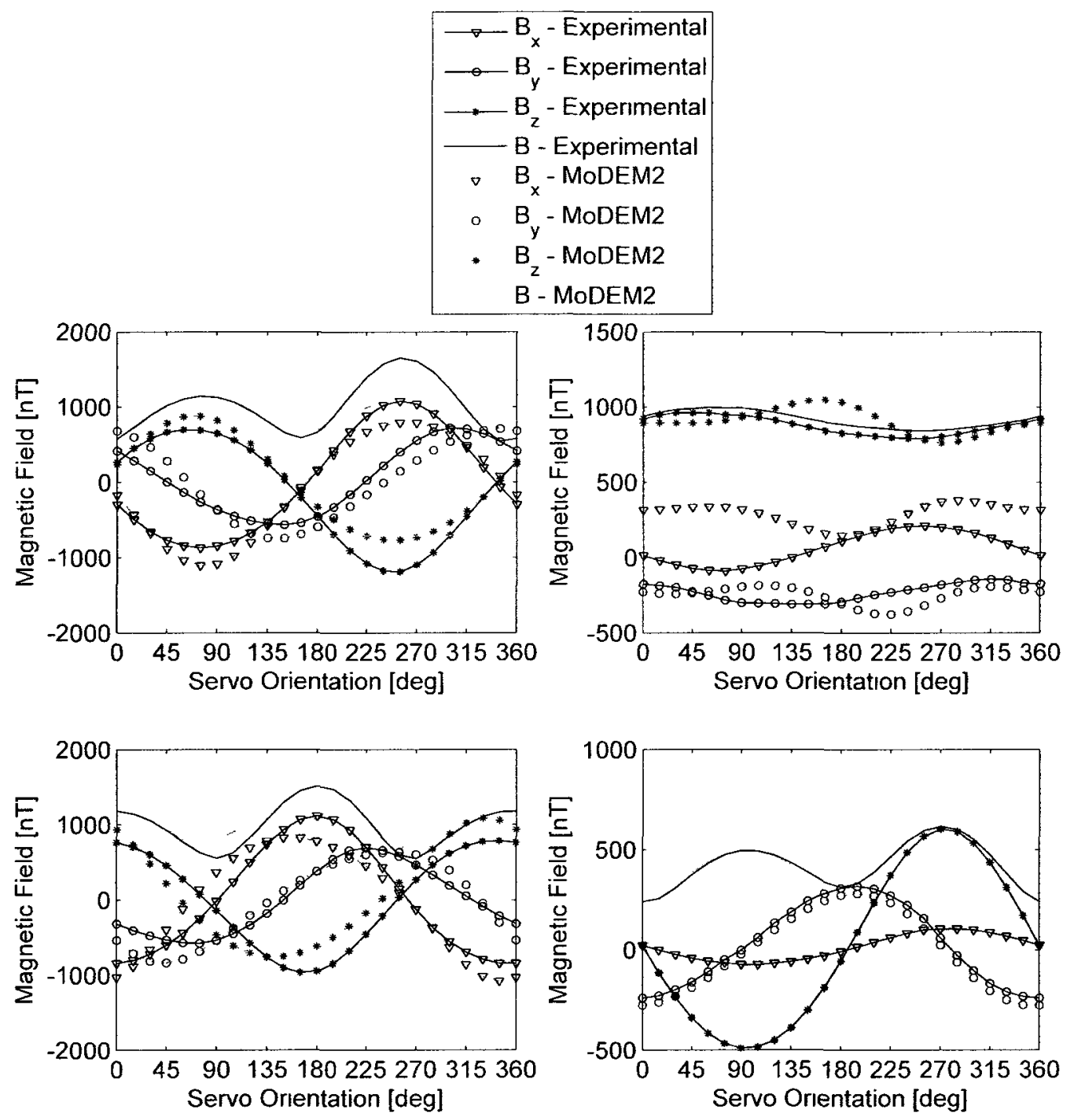

Figure 5.5: MoDEM2 characterization compared to experimental data collected in multiple measurement planes for a Hitec HSR-5990TG servo. Rotations about the $\mathrm{x}$-axis (top left), $\mathrm{y}$-axis (top right), and z-axis (bottom left) are shown for measurements taken at $0.22 \mathrm{~m}$ from the servo, as well as the data used for initial characterization (bottom right). The poor fit between the experimental and modelled curves for rotations about three separate measurement planes illustrates the need for additional data for characterization strategies. 
For Figures 5.4 and 5.5, the characterization was performed for the measurement plane modelled in Figures 5.1 and 5.3, illustrating that measurements in multiple planes are necessary to accurately model each magnetic field source in three dimensions. Additionally, if a single component of the position vector is zero, then the respective vector component of the magnetic field will not vary with rotation. Therefore, in order for all magnetic vector components to produce significant variations with rotation about the test axis, it is important to ensure that: a) at least two of the position vector components are at least twice as large as the respective component dimensions, and that b) the position vector is at least three times as large as the component's largest dimension. The mean absolute error for the MoDEM and MoDEM2 characterization schemes calculated from the three dimensional rotation data is shown in Table 5.1.

Table 5.1: Comparison of the mean absolute error for the MoDEM and MoDEM2 characterization schemes for measurements taken about three rotational axes at a distance of $0.22 \mathrm{~m}$. All units in $\mathrm{nT}$.

\begin{tabular}{|c|c|c|c|c|c|}
\hline $\begin{array}{c}\text { Characterization } \\
\text { Scheme }\end{array}$ & Servo & $e_{\vec{B}_{x}}$ & $e_{\vec{B}_{y}}$ & $e_{\vec{B}_{z}}$ & $e_{\| \vec{B}}$ \\
\hline \hline MoDEM & Hitec HSR-5990TG & 521.0 & 572.9 & 912.1 & 733.5 \\
\hline MoDEM2 & Hitec HSR-5990TG & 184.7 & 130.6 & 156.5 & 184.5 \\
\hline
\end{tabular}

The improved objective function and testing method led to the development of two novel characterization strategies, the Permanent Magnetic Dipole (PMaD) model and the Dual Permanent Magnetic Dipole (DuPMaD) model, which fit theoretical magnetic point dipole models to measurements collected in two or more planes. 


\subsection{Dual Permanent Magnetic Dipole Model}

The Dual Permanent Magnetic Dipole (DuPMaD) model was developed as an expansion to the MoDEM model to incorporate the revised objective function and measurement scheme. Similar to the MoDEM model, the DuPMaD model utilizes two magnetic point dipoles to represent each magnetic field source. Since the magnetic field at a point is the vector sum of all contributions, (2.5) may be expanded to include a second dipole as follows:

$$
\vec{B}=\frac{\mu_{o}}{4 \pi}\left[\frac{3\left(\vec{m}_{1} \cdot \hat{r}_{1}\right) \hat{r}_{1}-\vec{m}_{1}}{\left\|\vec{r}_{1}\right\|^{3}}+\frac{3\left(\vec{m}_{2} \cdot \hat{r}_{2}\right) \hat{r}_{2}-\vec{m}_{2}}{\left\|\vec{r}_{2}\right\|^{3}}\right],
$$

where $\vec{m}_{1}$ and $\vec{m}_{2}$ are the magnetic dipole moment vectors, and $\vec{r}_{1}$ and $\vec{r}_{2}$ are the position vectors for dipoles 1 and 2, respectively.

In the DuPMaD scheme, both dipoles have full degrees of freedom in their orientations and magnitudes, and are constrained to be located anywhere within the physical dimensions of the component being characterized. There are 12 parameters: $\vec{m}_{1, x}$, $\vec{m}_{1, y}, \vec{m}_{1, z}, \vec{m}_{2, x}, \vec{m}_{2, y}, \vec{m}_{2, z}, \vec{x}_{1}, \vec{y}_{1}, \vec{z}_{1}, \vec{x}_{2}, \vec{y}_{2}, \vec{z}_{2}$

In (5.3), the position vectors $\vec{r}_{1}$ and $\vec{r}_{2}$ must run from source to measurement point. If referring to the same geometry as defined in Figure 5.6, then the position vectors are calculated as follows:

$$
\vec{r}_{i=1,2}=-\left(\vec{r}_{c}+\vec{r}_{c m, i}\right),
$$

where $i$ denotes dipole 1 or 2 . The negative operator specifies that the position vector extends from source to measurement point. There will be no effect on the evaluation of (2.5) and (5.3) if the negative operator is not included, it is only used to specify the exact location of the measurement point. The position vectors $\vec{r}_{1}$ and $\vec{r}_{2}$ must 
be at least three times as large as the objects largest dimension: $L_{x}, L_{y}$, or $L_{z}$. The geometry used for the DuPMaD scheme is shown in Figure 5.6.

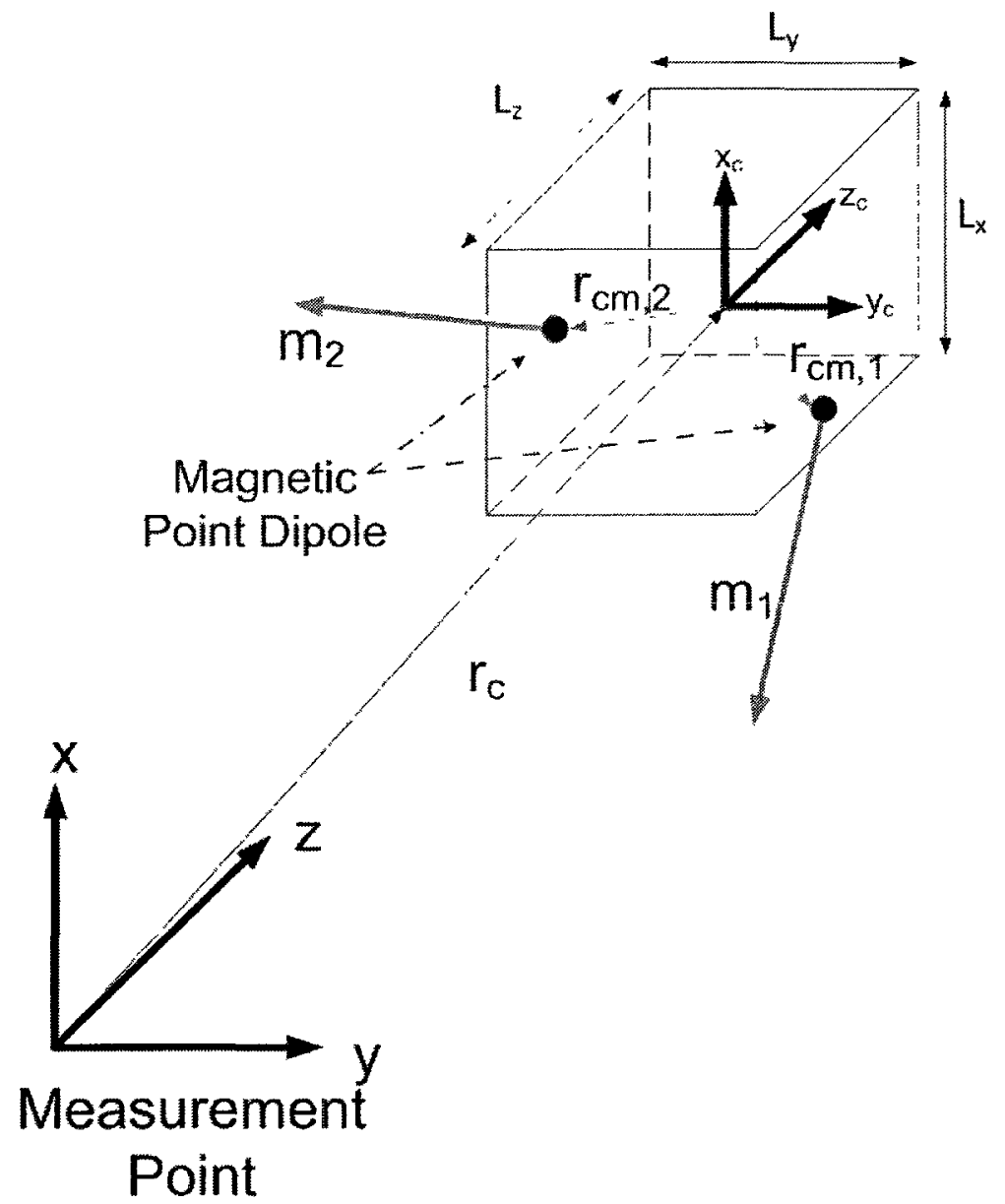

Figure 5.6: Geometry used for DuPMaD characterization.

Both MoDEM and DuPMaD use MATLAB's constrained nonlinear optimization algorithm, fmincon, to fit parameters to data collected experimentally. However, there are several key differences between the DuPMaD Model and the MoDEM model which are summarized in Table 5.2. 
Table 5.2: Model definition comparison for the MoDEM and DuPMaD models.

\begin{tabular}{|c|c|c|}
\hline & MoDEM & DuPMaD \\
\hline $\begin{array}{c}\text { Dipole } \\
\text { Moment }\end{array}$ & $\begin{array}{l}\text { The two point dipoles may } \\
\text { obtain any orientation } \\
\text { relative to the other, but } \\
\text { their magnitudes are } \\
\text { constrained such that the } \\
\text { major dipole, } \vec{m}_{1} \text {, must be } \\
\text { larger than the minor } \\
\text { dipole, } \vec{m}_{2} \text { : } \\
\qquad \vec{m}_{1} \geq \vec{m}_{2}\end{array}$ & $\begin{array}{l}\text { The magnitude of the two } \\
\text { point dipoles are free to } \\
\text { assume any magnitude or } \\
\text { orientation relative to one } \\
\text { another. }\end{array}$ \\
\hline $\begin{array}{l}\text { Dipole } \\
\text { Location }\end{array}$ & $\begin{array}{l}\text { The location of } \vec{m}_{1} \text { was } \\
\text { constrained such that it } \\
\text { must be within the physical } \\
\text { dimensions of the } \\
\text { component. The location } \\
\text { of } \vec{m}_{2} \text { was constrained such } \\
\text { that it was offset at some } \\
\text { distance, } Z_{\vec{m}_{1} \vec{m}_{2} \text { spacing } \text {, from }} \\
\vec{m}_{1} \text { in the } z \text {-direction of the } \\
\text { test component. } \\
Z_{\vec{m}_{1} \vec{m}_{2} \text { spacing was }} \\
\text { constrained to be within } \pm \\
0.01 \text { m. This allows for the } \\
\text { possibility of } \vec{m}_{2} \text { to be } \\
\text { placed outside of the } \\
\text { physical dimensions of the } \\
\text { component. }\end{array}$ & $\begin{array}{l}\text { The locations of } \vec{m}_{1} \text { and } \\
\vec{m}_{2} \text { were constrained such } \\
\text { that they must be within } \\
\text { the physical dimensions of } \\
\text { the component being } \\
\text { characterized. No } \\
\text { constraints were defined to } \\
\text { control the positions of } \vec{m}_{1} \\
\text { and } \vec{m}_{2} \text { relative to one } \\
\text { another. }\end{array}$ \\
\hline $\begin{array}{l}\text { Objective } \\
\text { Function }\end{array}$ & $\begin{array}{l}\text { Utilizes the MoDEM } \\
\text { objective function as } \\
\text { defined in Section } 5.2 \text {, } \\
\text { which fits only the total } \\
\text { field curves. }\end{array}$ & $\begin{array}{c}\text { Utilizes the MoDEM2 } \\
\text { objective function as } \\
\text { defined in Section } 5.2 \text {, } \\
\text { which fits the vector field } \\
\text { curves. }\end{array}$ \\
\hline $\begin{array}{c}\text { Experimental } \\
\text { Data }\end{array}$ & $\begin{array}{l}\text { Fits data taken about a } \\
\text { single axis in a single } \\
\text { measurement plane. }\end{array}$ & $\begin{array}{l}\text { Fits data taken about two } \\
\text { or more axes in one or } \\
\text { more measurement planes. }\end{array}$ \\
\hline
\end{tabular}


The DuPMaD model was used to characterize two servo actuators, the Hitec HSR5990TG and the Hitec HSM-7990TG, as can be seen in Figures 5.7 and 5.8, respectively. Figures 5.7 and 5.8 show that the DuPMaD characterization provides a better fit for the experimental data as a whole. This may be seen by comparing Figures 5.5 and 5.7, or by noting the mean absolute errors of each model as shown in Table 5.3.

Table 5.3: Comparison of the mean absolute error for the DuPMaD, MoDEM, and MoDEM2 characterization schemes for measurements taken about three rotational axes at a distance of $0.22 \mathrm{~m}$. All units in $\mathrm{nT}$.

\begin{tabular}{|c|c|c|c|c|c|}
\hline $\begin{array}{c}\text { Characterization } \\
\text { Scheme }\end{array}$ & Servo & $e_{\vec{B}_{x}}$ & $e_{\vec{B}_{y}}$ & $e_{\vec{B}_{z}}$ & $e_{\|\vec{B}\|}$ \\
\hline \hline MoDEM & Hitec HSR-5990TG & 521.0 & 572.9 & 912.1 & 733.5 \\
\hline MoDEM2 & Hitec HSR-5990TG & 184.7 & 130.6 & 156.5 & 184.5 \\
\hline DuPMaD & Hitec HSR-5990TG & 53.2 & 62.4 & 56.7 & 70.3 \\
\hline
\end{tabular}




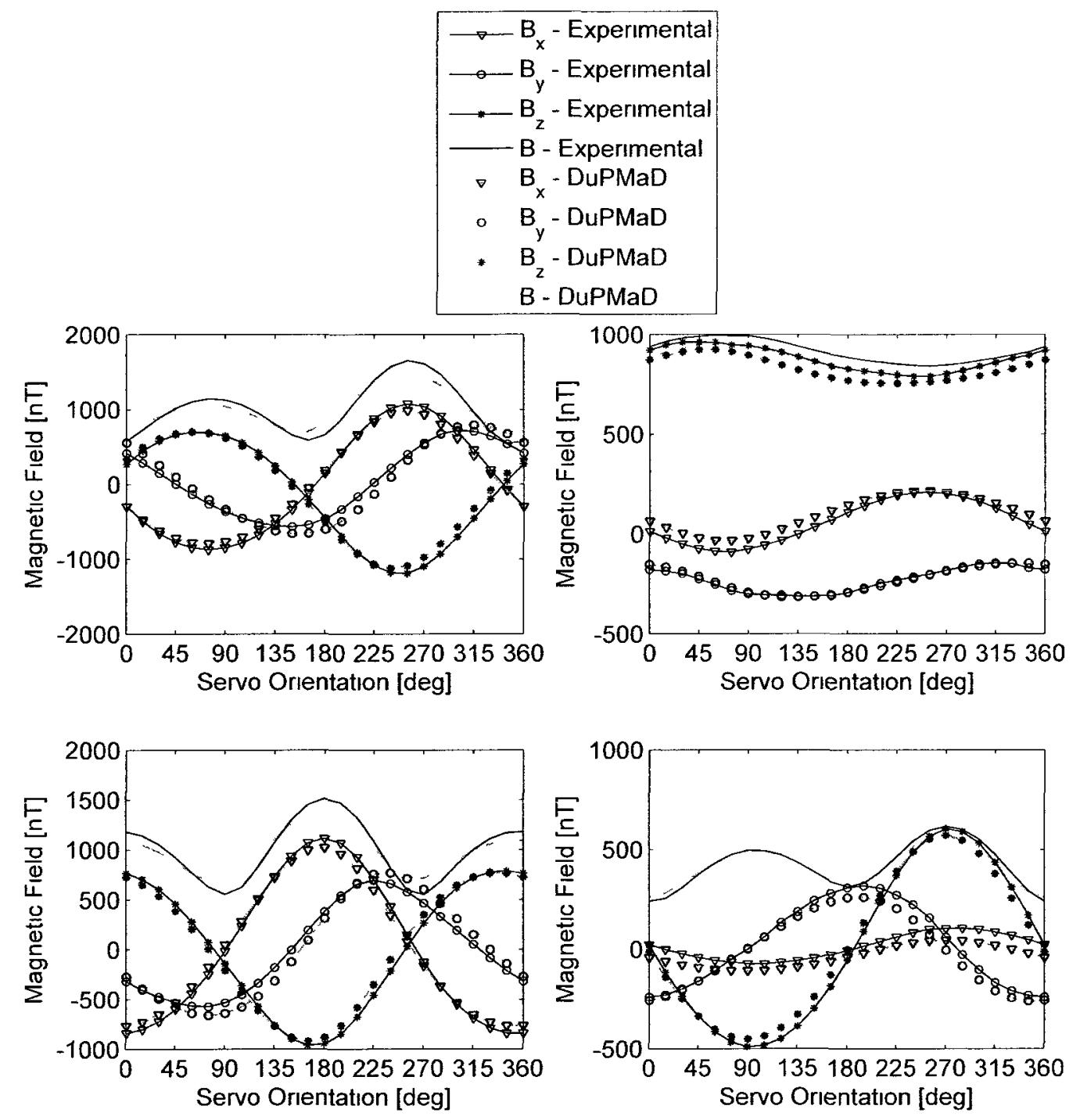

Figure 5.7: DuPMaD characterization of Hitec HSR-5990TG servo. Rotations about the $\mathrm{x}$-axis (top left), $\mathrm{y}$-axis (top right), and z-axis (bottom left) are shown for measurements taken at $0.22 \mathrm{~m}$ from the servo. Bottom right shows measurements taken at a distance of $0.30 \mathrm{~m}$, with rotation about the $\mathrm{x}$-axis of the servo. 

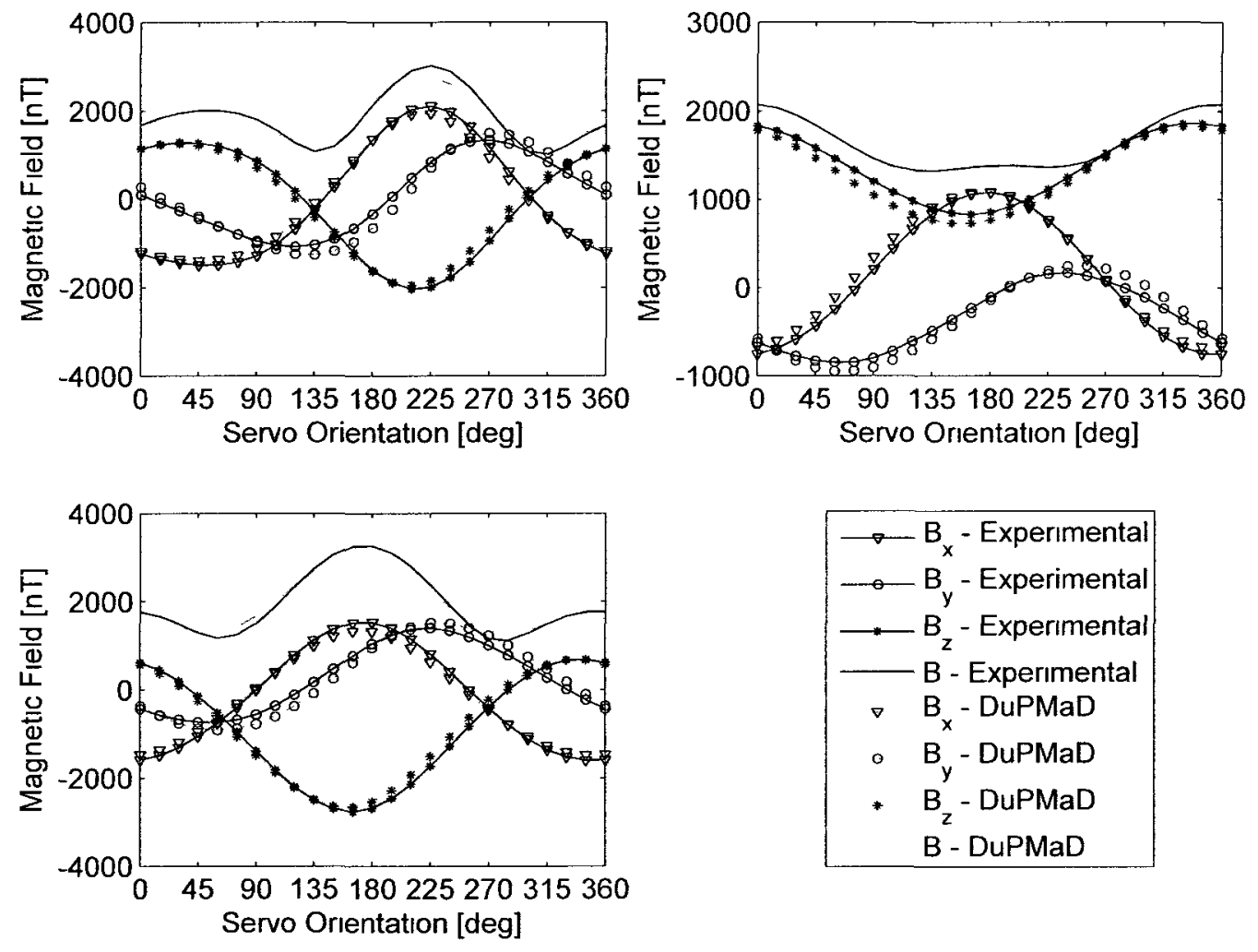

Figure 5.8: DuPMaD characterization of Hitec HSM-7990TG servo. Rotations about the $\mathrm{x}$-axis (top left), $\mathrm{y}$-axis (top right), and z-axis (bottom left) are shown for measurements taken at $0.22 \mathrm{~m}$ from the servo. 
If only fitting parameters to a single measurement plane such as in Figure 5.3, then the error between the modelled and experimental magnetic fields will be much lower for that measurement plane as can be seen in Figure 5.9, which is a comparison of Figures 5.3 and 5.7 (bottom right). Alternatively, if attempting to fit parameters to more than one measurement plane, there will be additional error between the experimental and modelled magnetic field for each measurement than there would be for a single measurement plane. This error is likely due to differences in the measurement conditions between data sets. However, additional measurement planes are necessary for proper characterization of all parameters, so this additional error is accepted in a trade-off between an accurate model that fits all three dimensions and a model that is highly accurate for only the measurement plane. 

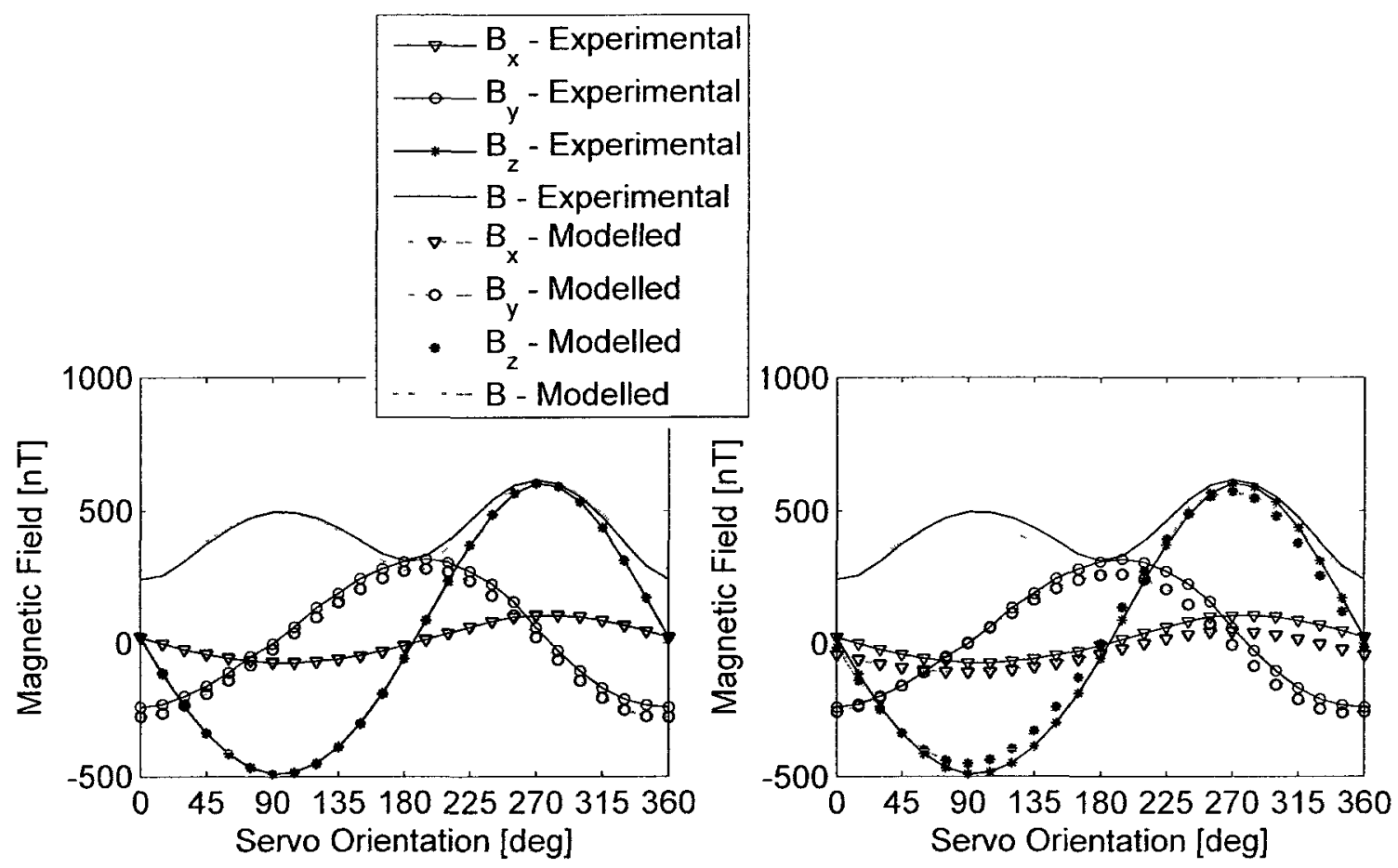

Figure 5.9: Comparison of MoDEM2 (left) and DuPMaD (right) characterization schemes for the Hitec HSR-5990TG servo rotating about the x-axis of the servo. Additional data from other rotational axis was used for the DuPMaD characterization causing a less accurate fit for the single rotational axis data shown, but a better fit for the data set as a whole as compared to the MoDEN2 strategy.

\subsection{Permanent Magnetic Dipole Model}

The Permanent Magnetic Dipole (PMaD) model was created as a sumplification to the DuPMaD model. It involves modelling sources using only a single magnetic point dipole located anywhere within the physical dimensions of the source, and assuming any orientation and magnitude. The motivation for this simplification was that sources modelled on GeoSurv II are all in the magnetic far-field, and therefore should be able to be modelled by a single magnetic point dipole [12]. The added complexity of using multipole sources(two or more dipoles) to model simple magnetic components will only marginally increase the accuracy of the model when modelling in the magnetic far-field. 
This model may be compared to the Direct Dipole Parameterization (DDP) scheme developed by Wells [1]. Wells' DDP scheme involved constraining a single point dipole to be at the centre of the component geometry, leaving only three parameters to be characterized: $\vec{m}_{x}, \vec{m}_{y}$, and $\vec{m}_{z}$. Additionally, the method used by DDP to characterize the three parameters involved calculating the magnetic dipole moment vector directly from (2.5), knowing both the magnetic field components and the position vector, at $N$ measurement points. This yielded $N$ different dipole moment vectors which were then averaged to produce a single estimate.

The DDP method involves an over simplification of the problem since it is unlikely that the magnetic dipole will be directly at the centre of the component geometry. Additionally, the method by which the dipole moment vector is calculated does not operate by attempting to reduce the error between the experimental and modelled magnetic fields, but instead attempts to directly calculate the model from the data.

The results of the PMaD characterization for the Hitec HSR-5990TG and the Hitec HSM-7990TG servos are shown in Figures 5.10 and 5.11, respectively. It can be seen that the PMaD model provides a good fit to the experimental data for both servos. 


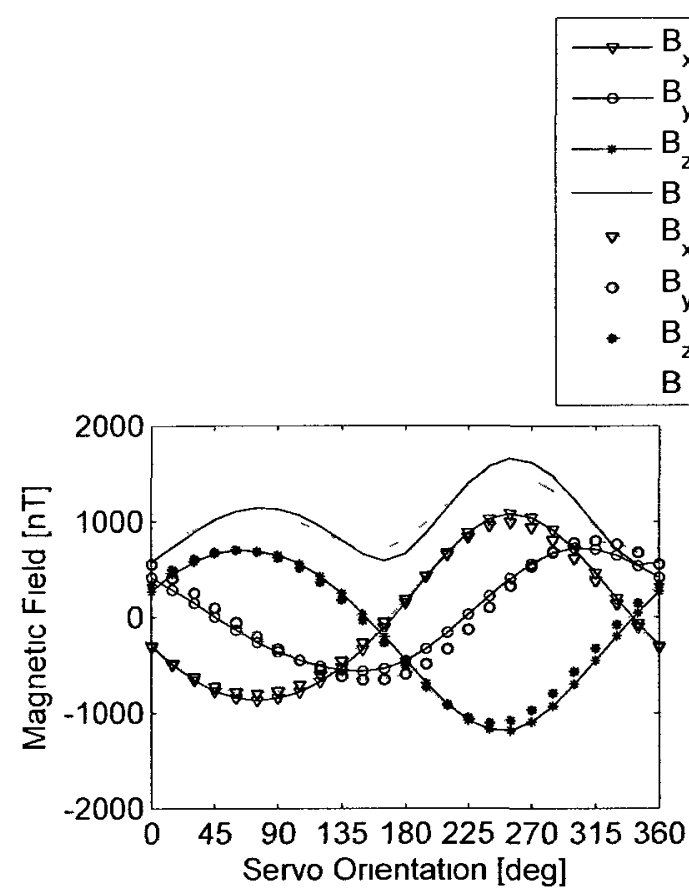

$x$ - Experimental $B_{y}-$ Experimental $B_{z}$ - Experimental

B - Experımental

- $B_{x}$ - DuPMaD

- $B_{y}$-DuPMaD

- $B_{z}$-DuPMaD

B - DuPMaD
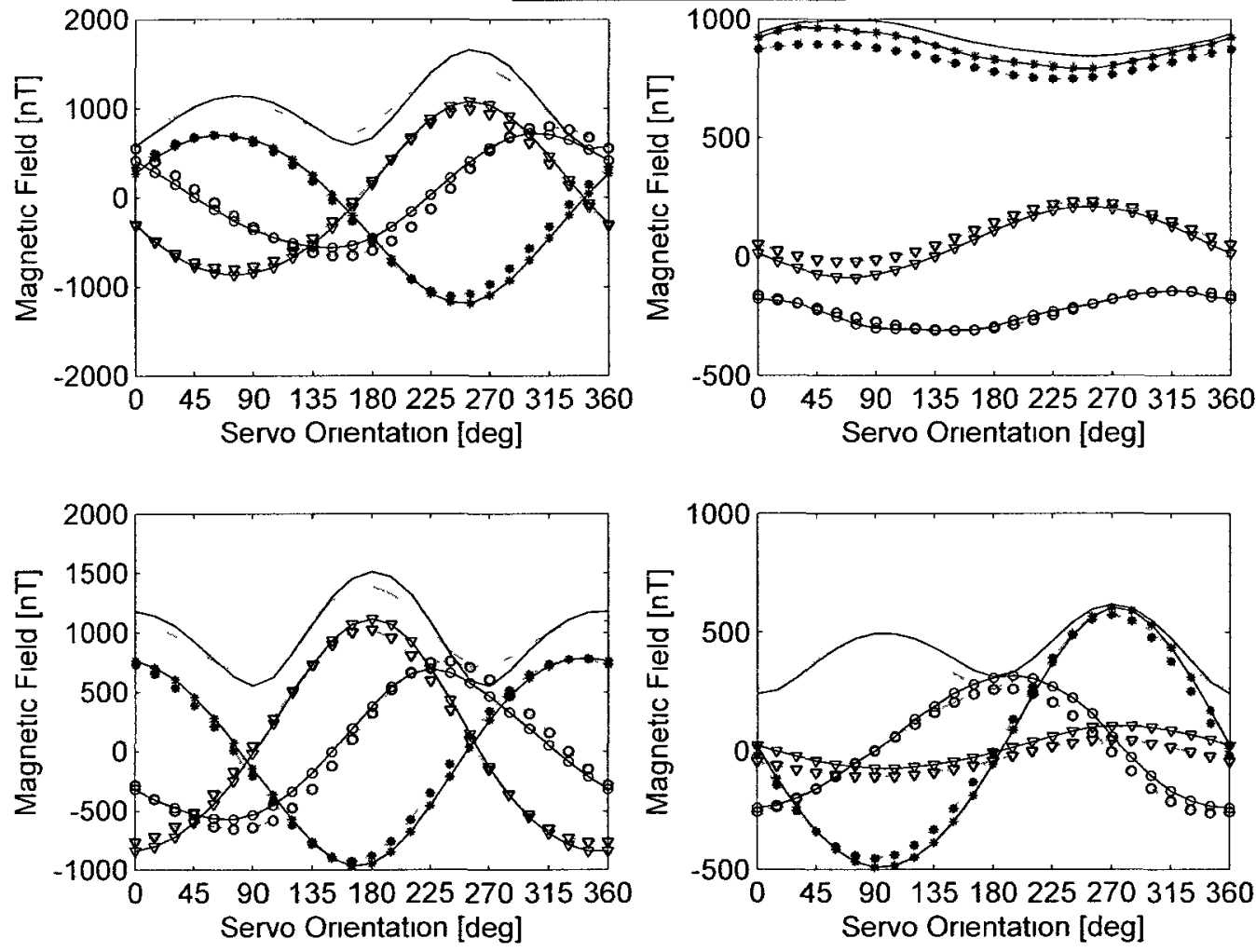

Figure 5.10: PMaD characterization of Hitec HSR-5990TG servo. Rotations about the $\mathrm{x}$-axis (top left), $\mathrm{y}$-axis (top right), and z-axis (bottom left) are shown for measurements taken at $0.22 \mathrm{~m}$ from the servo. Bottom right shows measurements taken at a distance of $0.30 \mathrm{~m}$, with rotation about the $\mathrm{x}$-axis of the servo. 

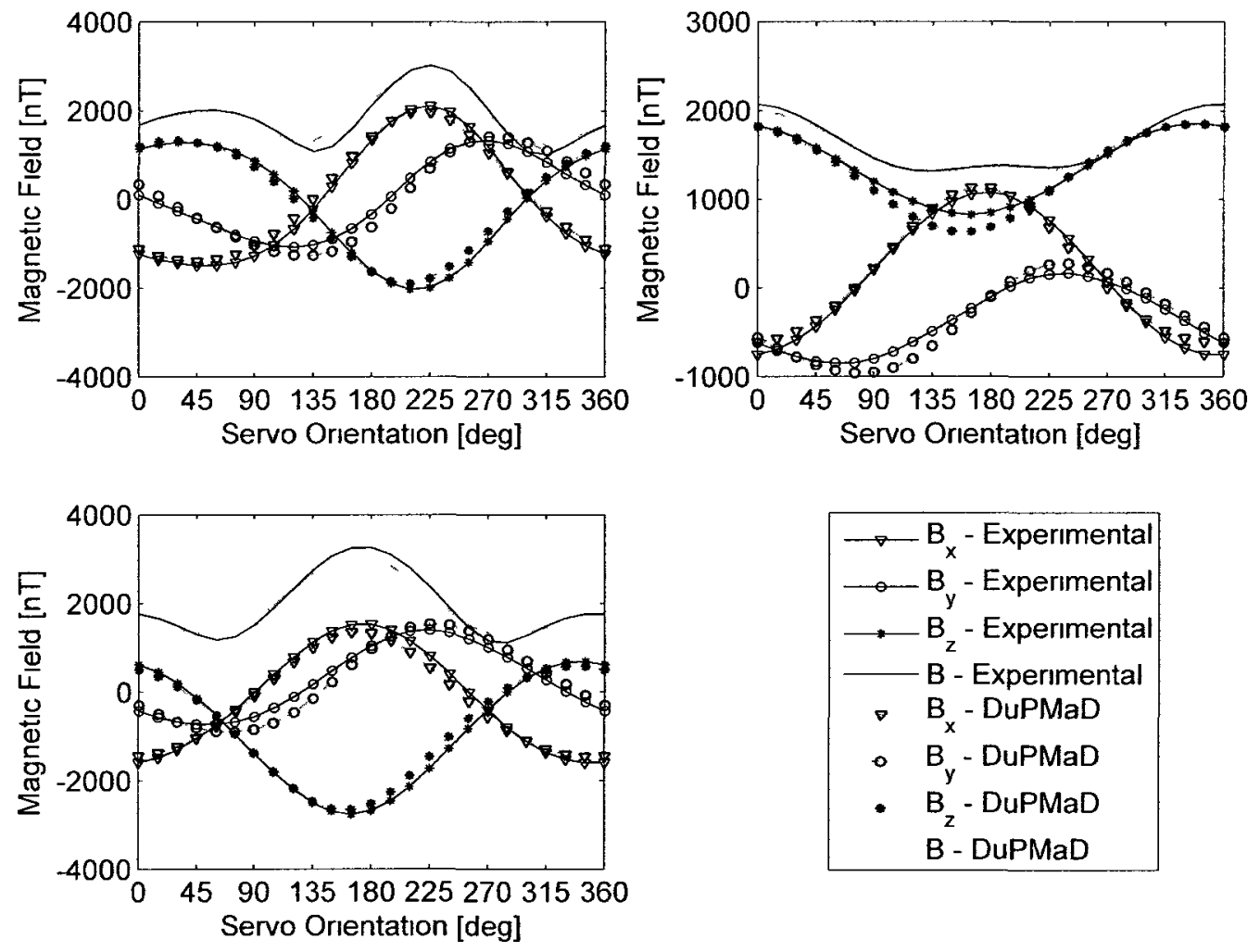

Figure 5.11: PMaD characterization of Hitec HSM-7990TG servo. Rotations about the $\mathrm{x}$-axis (top left), $\mathrm{y}$-axis (top right), and $\mathrm{z}$-axis (bottom left) are shown for measurements taken at $0.22 \mathrm{~m}$ from the servo. 
The mean absolute error for the MoDEM, MoDEM2, DuPMaD and PMaD characterization schemes for the Hitec HSR-5990TG and Hitec HSM-7990TG servos is shown in Table 5.4. The purpose of Table 5.4 is to compare the accuracy of the DuP$\mathrm{MaD}$ and the PMaD characterization schemes. Comparisons should not be drawn on the magnitude of the mean absolute error between the two servos since the magnetic fields they produce are not equivalent.

Table 5.4: Comparison of the mean absolute error for the DuPMaD and PMaD characterization schemes for measurements taken about three rotational axes at a distance of $0.22 \mathrm{~m}$. All units in $\mathrm{nT}$.

\begin{tabular}{|c|c|c|c|c|c|}
\hline $\begin{array}{c}\text { Characterization } \\
\text { Scheme }\end{array}$ & Servo & $e_{\vec{B}_{x}}$ & $e_{\vec{B}_{y}}$ & $e_{\vec{B}_{z}}$ & $e_{\| \vec{B}} \|$ \\
\hline \hline MoDEM & Hitec HSR-5990TG & 521.0 & 572.9 & 912.1 & 733.5 \\
\hline MoDEM2 & Hitec HSR-5990TG & 184.7 & 130.6 & 156.5 & 184.5 \\
\hline \multirow{2}{*}{ DuPMaD } & Hitec HSR-5990TG & 53.2 & 62.4 & 56.7 & 70.3 \\
\cline { 2 - 7 } & Hitec HSM-7990TG & 99.7 & 134.5 & 102.5 & 110.7 \\
\hline \multirow{2}{*}{ PMaD } & Hitec HSR-5990TG & 52.9 & 62.1 & 57.8 & 71.3 \\
\cline { 2 - 7 } & Hitec HSM-7990TG & 111.2 & 142.6 & 98.3 & 105.2 \\
\hline
\end{tabular}

From Table 5.4, it can noted that the mean absolute error is approximately the same for the DuPMaD scheme as it is for the PMaD scheme for both servos. This is to be expected: as the position vector increases, the effect of multipole sources, such as in the DuPMaD scheme, drop off at a rate faster than for dipole sources. Since the measurement points used for this calculation are in the magnetic far-field, the difference between a single dipole and a multipole representation should be negligible. This means that the DuPMaD scheme will also appear as a single dipole in the magnetic far-field, with some measure of error associated with the unaccounted multipole contribution. As was concluded by Zaffanella [12], this error decreases as 
the position vector increases. The location and orientation of the permanent dipole within the servo casing geometry, for the Hitec HSR-5990TG, is shown in Figure 5.12.

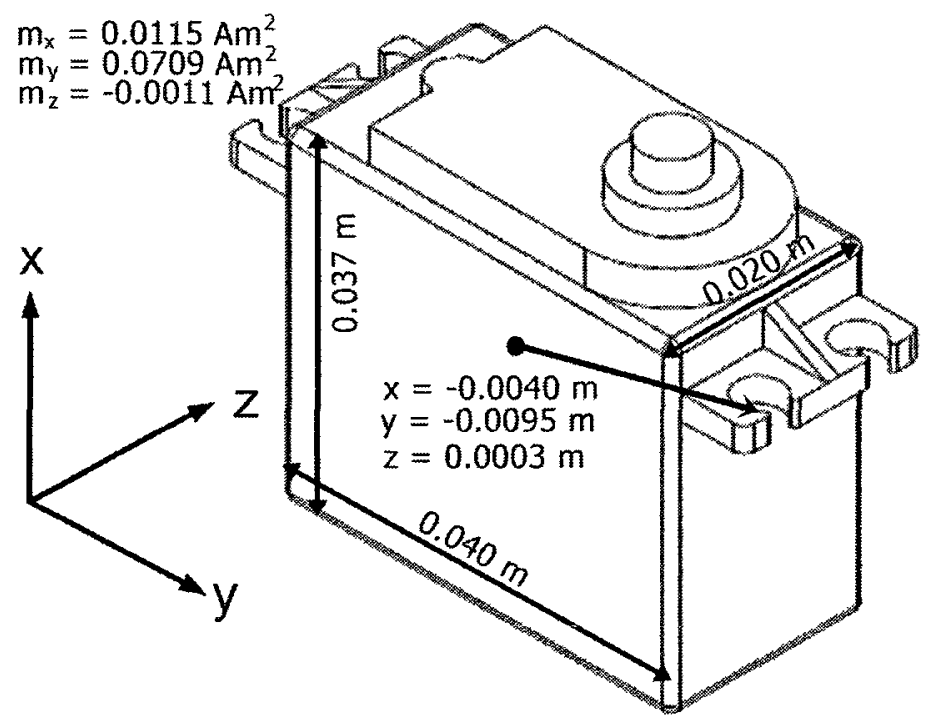

Figure 5.12: Illustration of magnetic dipole location and orientation within the Hitec HSR-5990TG servo casing.

\subsection{Validation using Finite Element Method Anal- ysis}

In order to verify the DuPMaD and PMaD characterization schemes, the Hitec HSR-5990TG servo was modelled using COMSOL Multiphysics, a finite element analysis software. This was done to verify that the physics used for the dipole characterization schemes (DuPMaD and PMaD) are realistic, and that the dipole schemes are adequate to represent simple magnetic sources.

Two concentric spheres, with their centres at the geometrical centre of the servo, were generated to represent the two domains of the model. Infinite elements were used for the outer domain, defined as the volume between the boundary of the larger sphere 
and the smaller sphere. Infinite elements account for the magnetic fields extending to infinity by providing a means for the magnetic flux to go from positive infinity to negative infinty. The inner domain which was defined as the volume of the smaller sphere. was modelled as free-space where the magnetic flux could circulate freely.

Each magnetic dipole was represented by a single point, which was then assigned a moment vector using the magnetic point dipole feature found in COMSOL's AC/DC module. The location. magnitude, and orientation of each point dipole was defined by the DuPMaD and PMaD characterization schemes. Additionally, measurement points were created around the servo to allow for the collection of data similar to those collected experimentally. The measurements points were specified to simulate those taken in Figure 4.7 for direct comparison with experimental data, and magnetic fields modelled using characterization schemes.

Figure 5.13 shows the comparison of the experimental magnetic total field values to the DuPMaD and PMaD characterization data generated in MATLAB and COMSOL. The conclusion can then be drawn that the physics used in the dipole modelling schemes do indeed match those of reality as simulated using COMSOL. Additionally, it may be noted that the DuPMaD and PMaD schemes produce almost identical magnetic fields at the measurement points, which are closely matched by their COMSOL counterparts. 

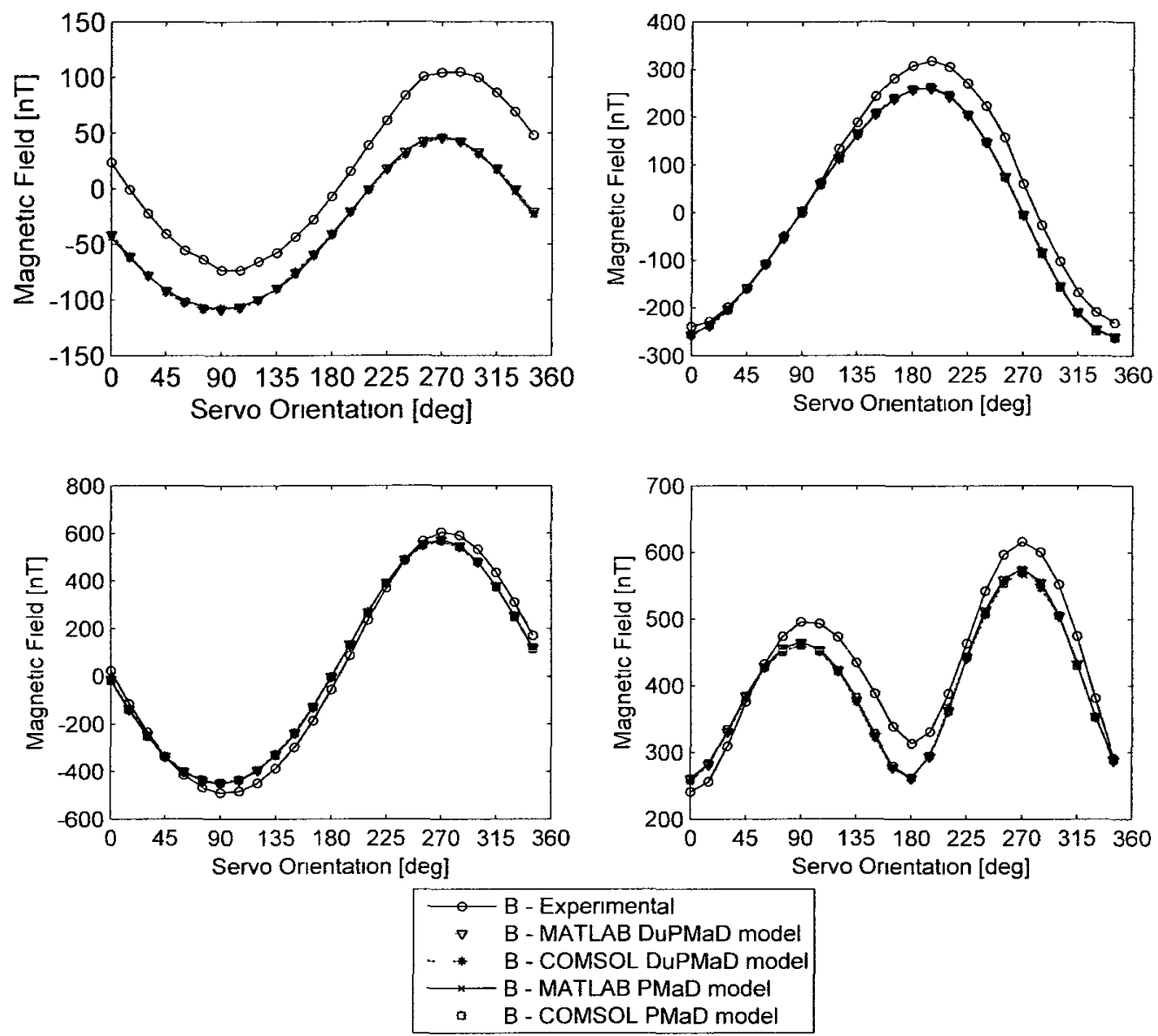

Figure 5.13: Comparison between experimental data and theoretical results produced by MATLAB and COMSOL for the DuPMaD and PMaD characterization schemes. Shown are $\vec{B}_{x}$ (top left), $\vec{B}_{y}$ (top right), $\vec{B}_{z}$ (bottom left), and $\|\vec{B}\|$ (bottom right) for all cases. It can be seen that the simulated physics using COMSOL provides a very close fit to the physics modelled in MATLAB for both characterization schemes.

For the GeoSurv II application. most magnetic field sources are considered to be in the magnetic far-field. meaning that they can be accurately modelled using a single magnetic dipole. Therefore, since the DuPMaD and PMaD models both have similar associated error, the simplicity of the PMaD model makes it the characterization scheme of choice for the source configuration optimization and cancellation models. 


\subsection{Modelling Complex Magnetic Sources}

Complex magnetic sources may include: a) large sources which consist of primarily permanent magnet and/or electromagnet sources, or b) sources consisting of ferromagnetic materials. Sources consisting primarily of permanent magnet and/or electromagnet sources may be modelled by expanding simple dipole models to incorporate additional dipoles such as with the DuPMaD characterization scheme. This is relatively straight forward, and the results for a characterization of the engine gradiometer data are shown in Figure 5.14 for a scheme involving four permanent magnetic dipoles.

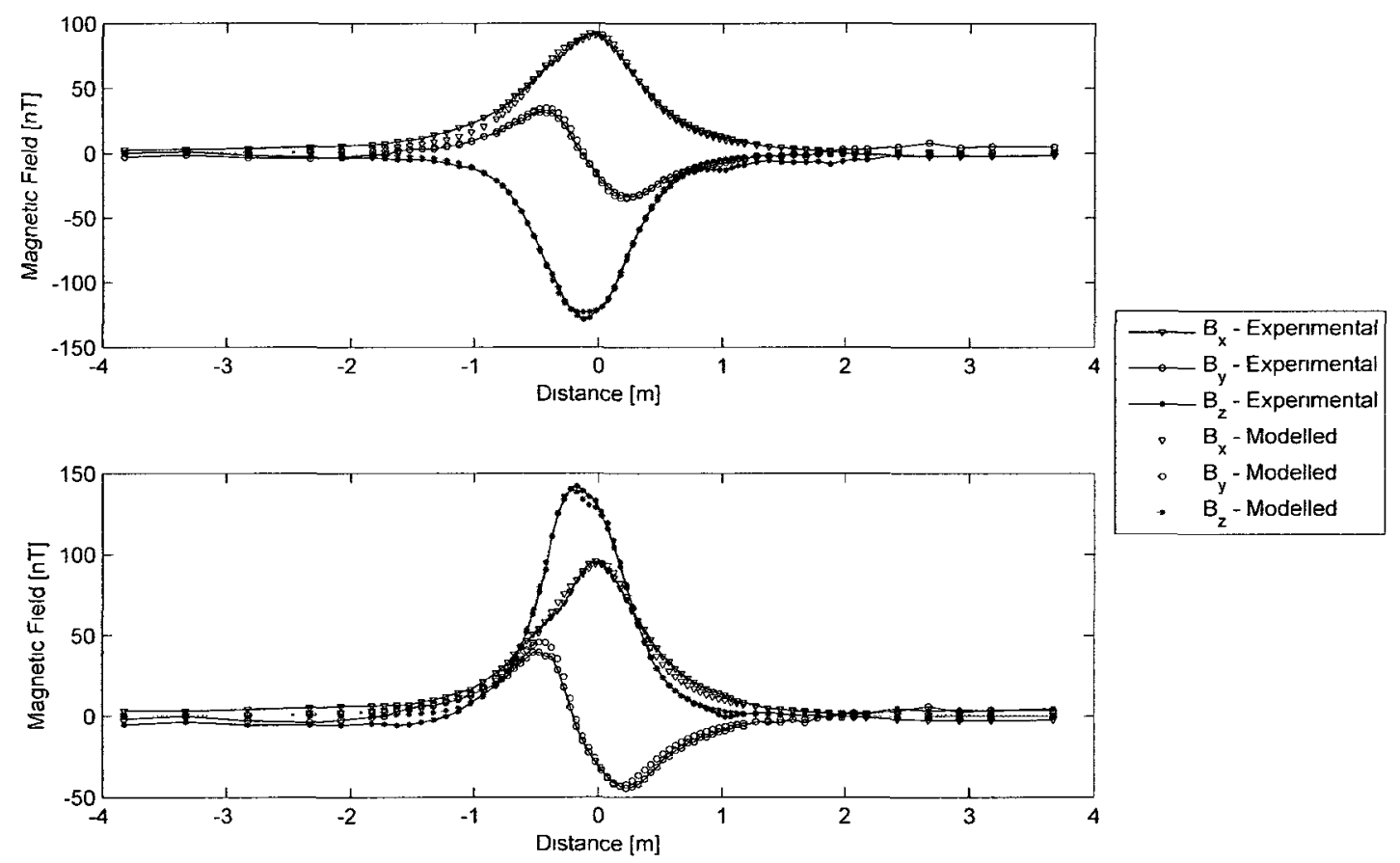

Figure 5.14: Characterization of engine using four permanent magnetic dipoles. Shown are the results for the starboard (top) and port (bottom) fluxgates. This characterization strategy yields an excellent fit to experimental data, but is not reliable due to the nature of ferromagnetic materials. This characterization is only reliable for the state of the engine at the time of testing, and for the heading and background magnetic field present during testing. 
Modelling sources consisting of ferromagnetic materials is complex and requires knowledge of the exact state and history of the material. Magnetic materials will behave as a collection of magnetic dipoles, each representing the present magnetization of the net effect of many domains. However, the dipoles used to represent ferromagnetic material are not permanent and are dependent on the applied H-field, and magnetization of the material at a given time. It is possible to model ferromagnetic materials using permanent magnetic dipoles, but this approach will yield models that become invalid as soon as the magnetic history of the material changes. Variations in the H-field, thermal fluctuations, straining the material, and several other factors must all be accounted for in the material's magnetic history [4]. More often than not, the magnetic history of a material will cause it to become more magnetic over time, which makes ferromagnetic materials a transient source of magnetic noise.

This topic has been studied for the application of reducing a ship's magnetic signature as outlined by Holmes [32]. The models generated in [32] apply mainly to a ferromagnetic ship's hull, and would not be able to capture the magnetic history that is present in the GeoSurv II engine components.

Since it is difficult to accurately track all parameters that affect the magnetization of a material, it is easier to simply replace ferromagnetic components with nonmagnetic equivalents, and to position any remaining ferromagnetic material as far away from magnetometers as possible. If ferromagnetic materials cannot be eliminated, they should be monitored and degaussed when necessary as was described in Section 2.2.2.

\subsection{Characterization Conclusions}

Two new characterization schemes were presented in this chapter. The DuPMaD strategy utilizes two permanent magnetic dipoles, and the PMaD strategy utilizes a 
single permanent magnetic dipole to model simple magnetic noise sources. Two servo actuators, the Hitec HSR-5990TG and the Hitec HSM-7990TG, were both characterized by fitting the PMaD and DuPMaD models to experimental data. The results of the characterization were validated using the COMSOL Multiphysics software. The DuPMaD and PMaD models show an improved characterization compared to the previously developed MoDEM model [1]. It was found that both the PMaD and DuPMaD produce nearly identical results, therefore the PMaD model was selected for use in cancellation and optimization strategies due to its increased simplicity. 


\section{Chapter 6}

\section{CMC Passive Cancellation Strategy}

\subsection{Introduction}

Magnetic signature reduction of all individual components would lead to a decrease in the magnetic noise at the wingtip magnetometers. Since it is not feasible to simply reduce the magnetic signature of off-the-shelf components such as servos without altering their performance, it is necessary to use cancellation strategies. Cancellation strategies aim to reduce the far-field magnetic signature of components through the use of some additional magnetic component, as was outlined in Section 2.5.4. The strategy discussed in this chapter will outline the use of a cancellation magnet to rcduce the far-field effect of static magnetic noise sources. A method to optimally position and orient each cancellation magnet relative to the respective source will be presented and experimentally validated.

\subsection{Development of Passive Cancellation Strategy}

Since the magnetic field at any point is the vector sum of the effect of all magnetic noise sources, it is possible to have a smaller net magnetic field for multiple sources than it is for sources acting individually. This property is exploited in the passive 
cancellation strategy developed here, known as the Cancellation Magnet Configuration (CMC) strategy. The CMC strategy involves optimizing the configuration of a permanent magnet relative to a source, such as a servo, with the objective of minimizing the net magnetic far-field. A model of both the magnet and the source must be used for this strategy, which may be obtained using characterization strategies outlined in Chapter 5.

The permanent magnets used in this strategy were from a circular magnet sampler containing magnets of five different sizes with lift strengths ranging from 11.1 to 40 $\mathrm{N}$, available from Lee Valley. Since it is difficult to assign a coordinate system for one circular magnet that is easily transferable to a similar magnet, the magnetic moment vector of each magnet was assumed to be oriented along the axial direction as shown in Figure 6.1. This is a reasonable assumption given the properties of circular magnets, and was confirmed through experimental results which showed no variation in magnetic field values with rotation when performing rotation testing (refer to Section 4.2.2) about the axial direction of each magnet.

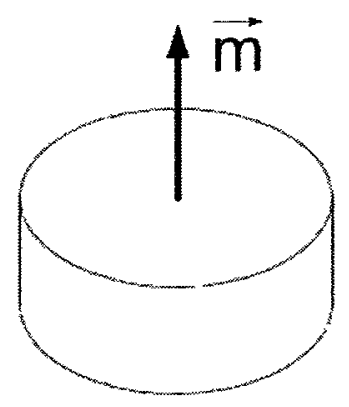

Figure 6.1: Direction of magnetic moment vector for permanent magnet used in CMC strategy. The magnitude of the dipole moment vector was determined through a modified version of the PMaD characterization strategy. 
The objective function of this strategy is to minimize the sum of the total magnetic field values at a predefined set of points in the magnetic far-field region. The near-field region will have multipole components created by the proximity of the cancellation magnet to the source, which will cause a larger magnetic near-field. As was mentioned in Section 2.3, the magnetic field decreases a rate proportional to $1 /\|r\|^{n}$ where $n=3$ for dipole sources and $n \geq 4$ for multipole sources. so if evaluating the magnetic field in the far-field region, the multipole components may be assumed to be negligible. Since the CMC strategy optimizes based on minimizing the magnetic field at a series of points, it is important to evaluate points only in the magnetic far-field region to avoid contaminating the CMC metric, which is defined as follows:

$$
\|\vec{B}\|_{\text {total }}=\min \left(\sum_{i=1}^{n}\left\|\vec{B}_{i}\right\|\right)
$$

where $\left\|\vec{B}_{\imath}\right\|$ is the total magnetic field at point $i$. The objective function, $\|\vec{B}\|_{t o t a l}$, is evaluated at $n$ points around each source. Rather than minimizing the net magnetic field at a single distance, evaluation points were specified at various distances to attempt to negate any near field effects. The evaluation points defined for the HSR5990TG servo in the CMC strategy were specified to simulate measurement points on the MMTA-3D test apparatus at -z-distances spanning $0.18 \mathrm{~m}$ to $0.60 \mathrm{~m}$ in 0.06 $m$ increments. The algorithm used for the CMC strategy is MATLAB's constrained nonlinear optimization algorithm, fmincon.

Constraints were included in the CMC algorithm to prevent the permanent magnet from being placed within the geometry of the source, as can be found in the script titled CMCconstraint.m. A value for the allowable distance for the cancellation magnetic to be placed from each side of the source was defined, and treated as bounds for the optimization algorithm. Additionally, the orientation of the cancellation magnet 
was required to be normal to all coordinate axes of the sources to simplify placement.

The CMC algorithm operated by optimizing the position of different cancellation magnet orientations for each of five previously characterized permanent magnets. The best solution was stored and treated as the optimized result for all orientations and magnet sizes as can be seen in the script CMCmaster.m.

\subsubsection{Results from CMC Implementation}

The CMC strategy was implemented to reduce the magnetic signature of the Hitec HSR-5990TG servo. Models generated using the PMaD characterization scheme of both the servo and the permanent magnet were used to determine the optimal position and orientation of the permanent magnet with respect to the the servo. A magnet mount, shown in Figure 6.2 was designed to allow for the precise positioning of the cancellation magnet relative to the servo, and was produced using a rapid prototyping machine. The experimental procedure and apparatus followed exactly that of Section 4.3.2, with-z-distances of $0.18 \mathrm{~m}, 0.30 \mathrm{~m}$, and $0.50 \mathrm{~m}$ used for the MMTA-3D test apparatus, as can be seen in Figure 6.3. The purpose of this set of experiments was to determine if the addition of the cancellation magnet would indeed cause a reduction in the magnetic signature of the servo. 


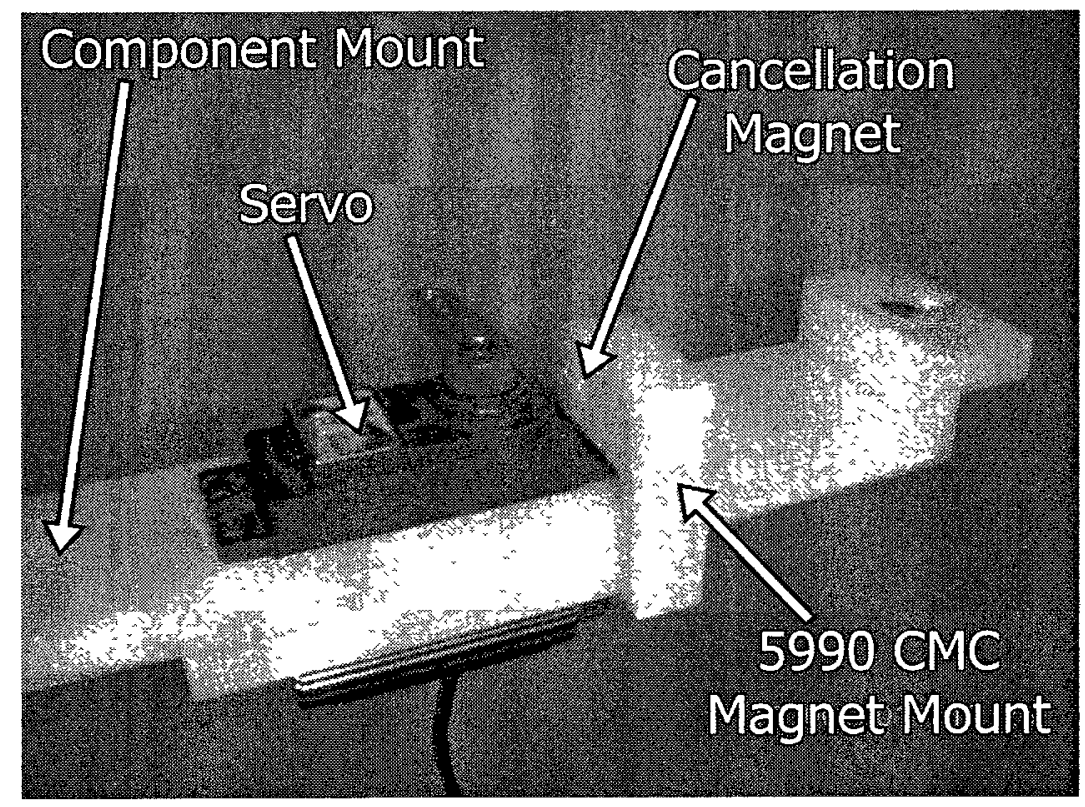

Figure 6.2: Magnet mount constructed to hold cancellation magnet for the Hitec HSR-5990TG servo. Part was manufactured on a rapid prototyping machine to allow for precise positioning and orientation of the cancellation magnet.

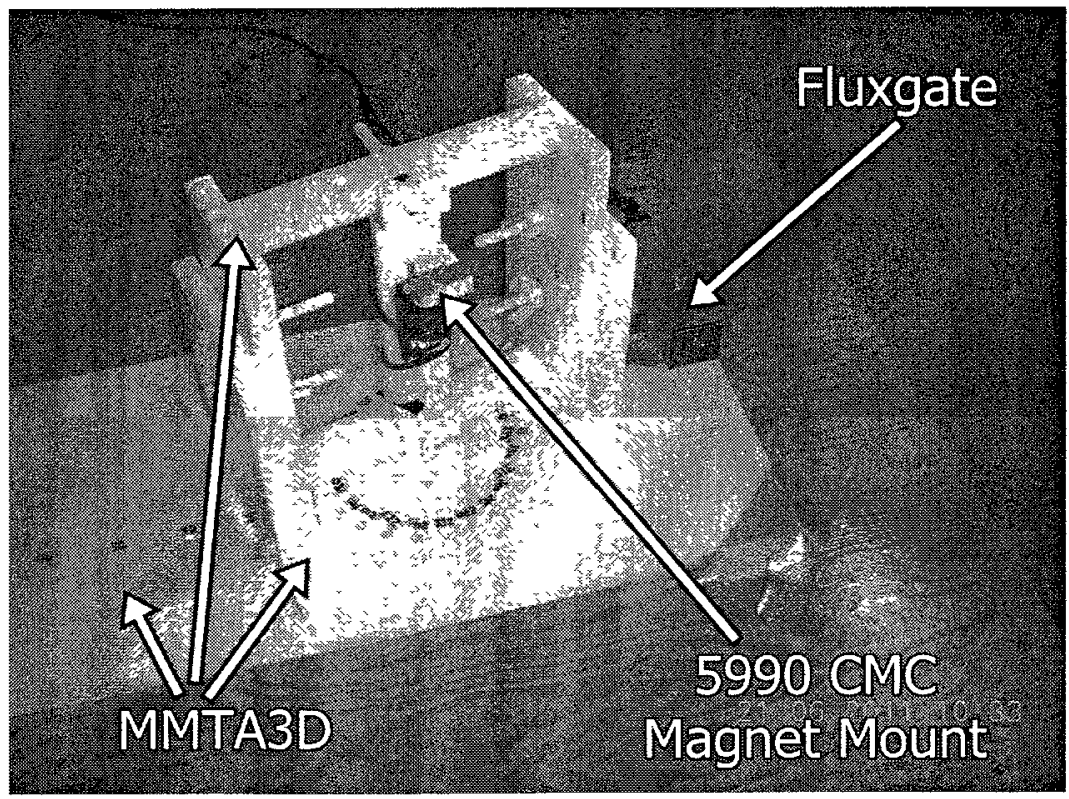

Figure 6.3: Experimental setup used for CMC validation. Shown is the Hitec HSR$5990 \mathrm{TG}$ for the $\mathrm{y}$-axis rotation configuration on the MMTA-3D at a -z-distance of $0.18 \mathrm{~m}$. 
The theoretical results generated in the MATLAB model show a significant reduction in the magnetic field with the CMC strategy. This may be seen in Figures 6.4 to 6.6 which compare the total field values of the Hitec HSR-5990TG servo with and without the CMC strategy for various distances and rotational axes.
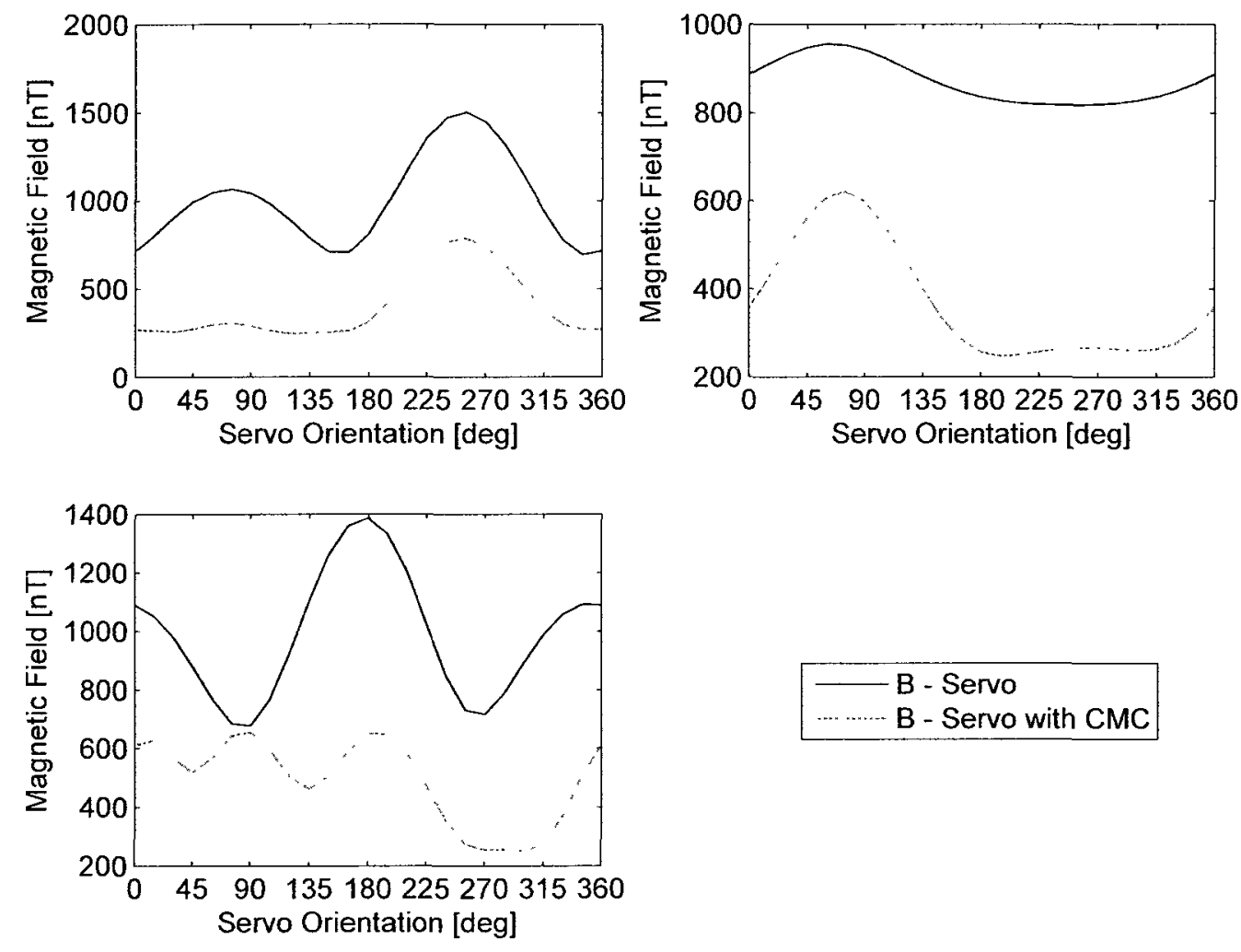

Figure 6.4: Theoretical CMC results for the Hitec HSR-5990TG servo at a distance of $0.22 \mathrm{~m}$. Shown are rotations about the servo's x- (top left), $\mathrm{y}$ - (top right), and $\mathrm{z}$ - (bottom left) axis. The total magnetic field values are shown, indicating a reduction in the magnetic signature with the CMC strategy. 

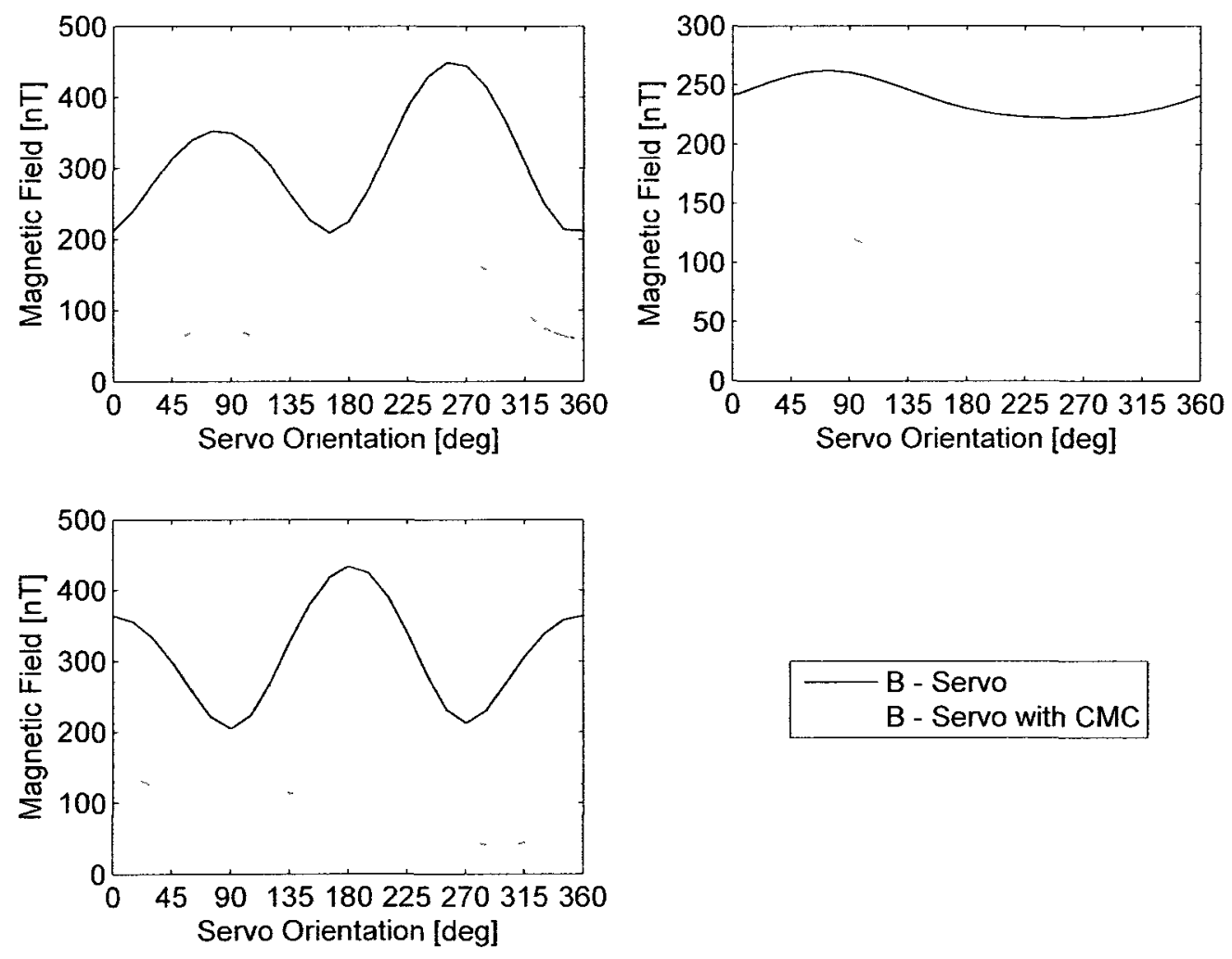

B - Servo

B - Servo with CMC

Figure 6.5: Theoretical CMC results for the Hitec HSR-5990TG servo at a distance of $0.33 \mathrm{~m}$. Shown are rotations about the servo's x- (top left), y- (top right), and z- (bottom left) axis. The total magnetic field values are shown, indicating a reduction in the magnetic signature with the CMC strategy. 

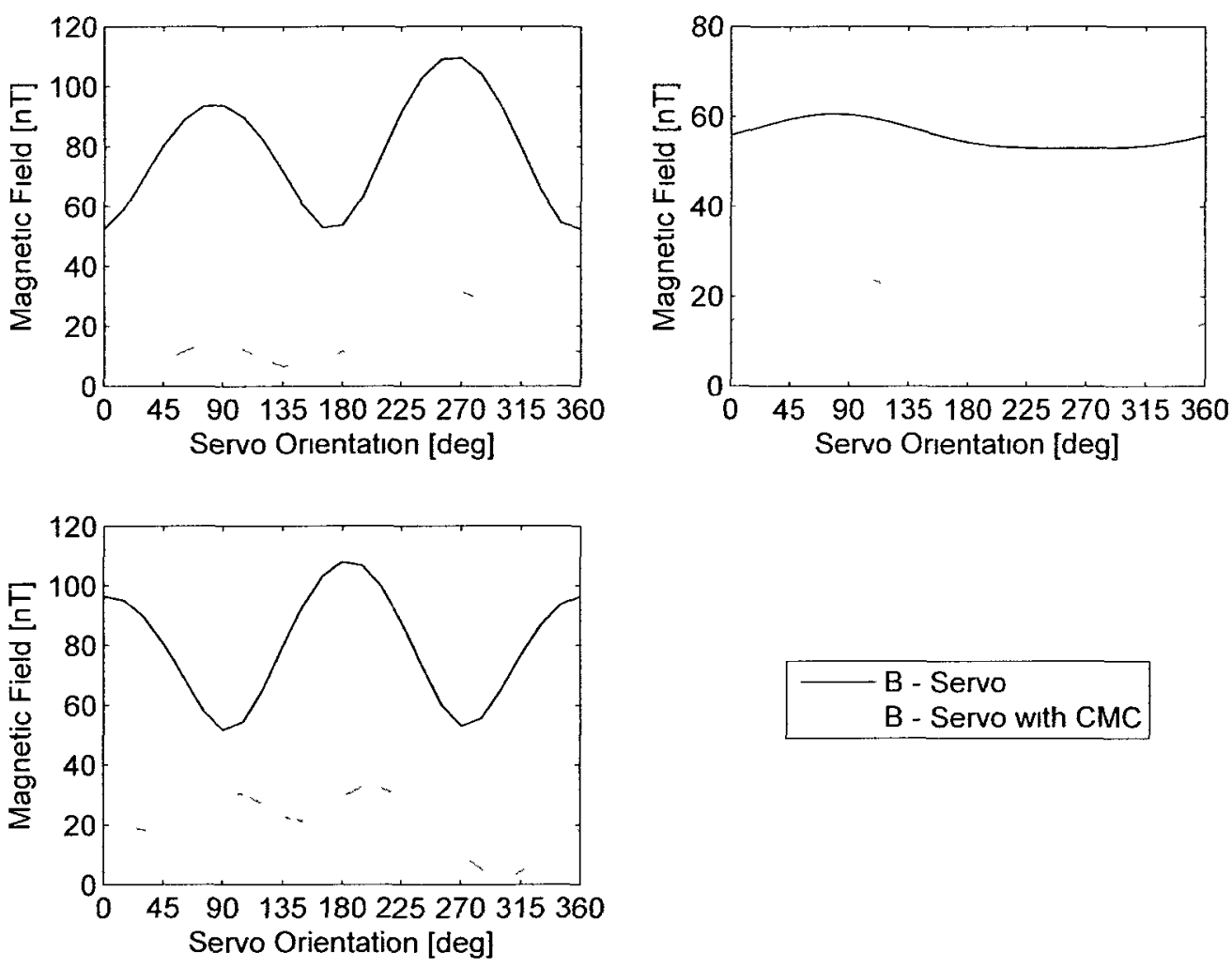

Figure 6.6: Theoretical CMC results for the Hitec HSR-5990TG servo at a distance of $0.52 \mathrm{~m}$. Shown are rotations about the servo's $\mathrm{x}$ - (top left), $\mathrm{y}$ - (top right), and $\mathrm{z}$ - (bottom left) axis. The total magnetic field values are shown, indicating a reduction in the magnetic signature with the CMC strategy. The magnetic signature is even further reduced than it is at a distance of $0.22 \mathrm{~m}$ (Figure 6.7), due to the multipole effects reducing rapidly with distance. 
Figures 6.4 to 6.6 may be compared to results measured experimentally, shown in Figures 6.7 to 6.9 for distances in the -z-direction of MMTA-3D of $0.18 \mathrm{~m}, 0.30 \mathrm{~m}$ and $0.50 \mathrm{~m}$, respectively.
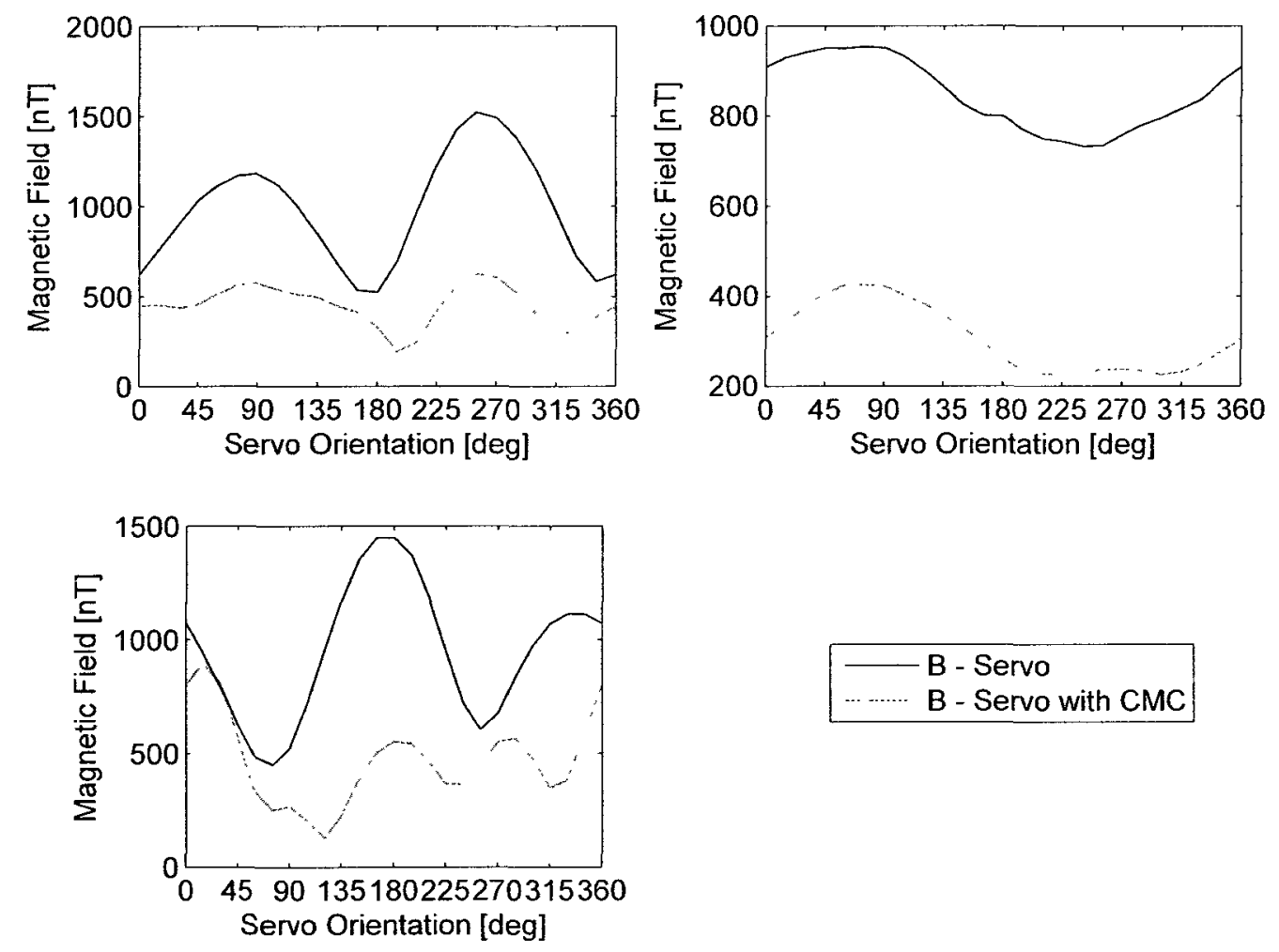

Figure 6.7: Experimental CMC results for the Hitec HSR-5990TG servo at a distance of $0.22 \mathrm{~m}$. The total magnetic field data are shown for rotations about the servo's $\mathrm{x}$ - (top left), $\mathrm{y}^{-}$(top right), and $\mathrm{z}$ - (bottom left) axis. 

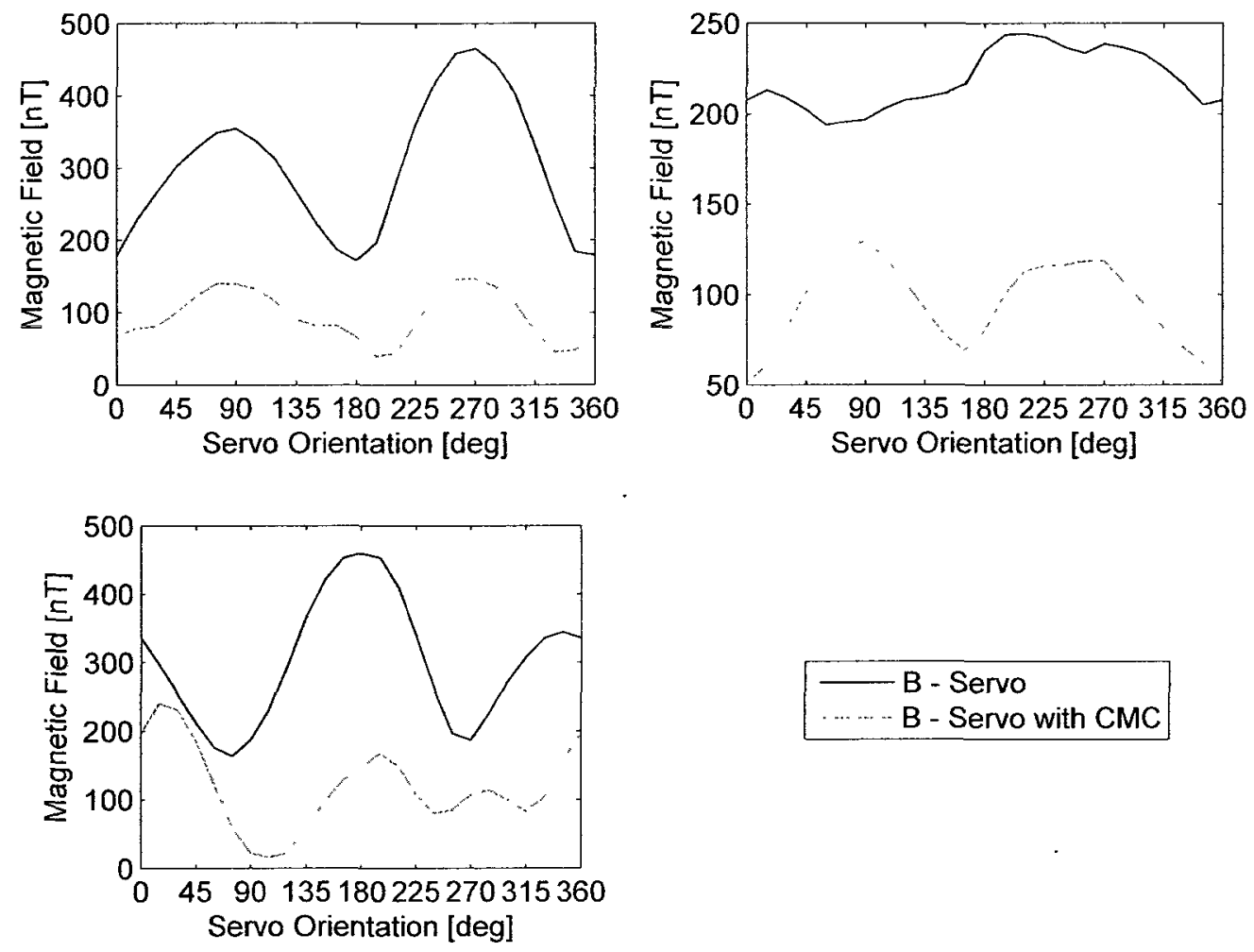

Figure 6.8: Experimental CMC results for the Hitec HSR-5990TG servo at a distance of $0.33 \mathrm{~m}$. The total magnetic field data are shown for rotations about the servo's $\mathrm{x}$ - (top left), $\mathrm{y}$ - (top right), and z- (bottom left) axis. 

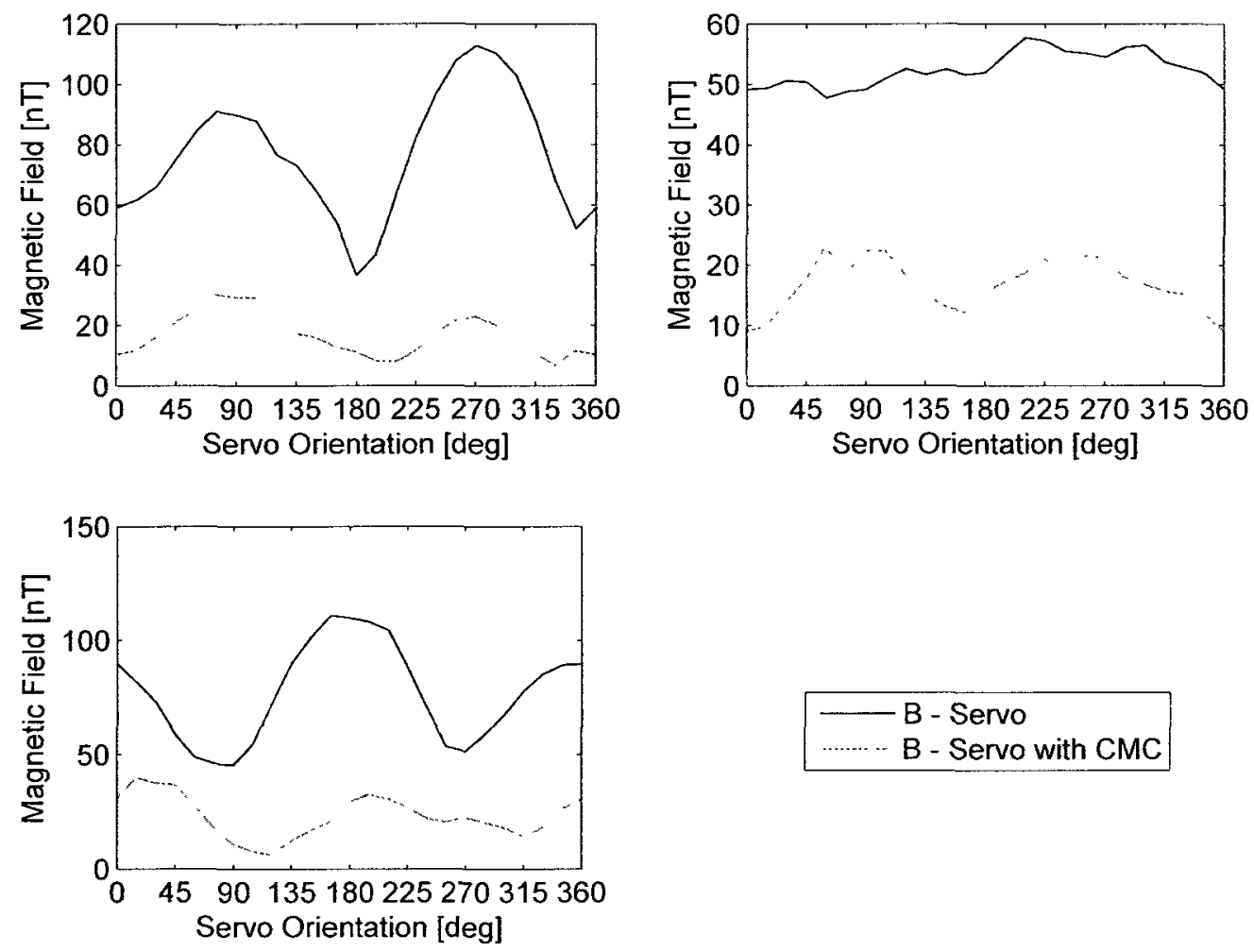

Figure 6.9: Experimental CMC results for the Hitec HSR-5990TG servo at a distance of $0.52 \mathrm{~m}$. The total magnetic field data are shown for rotations about the servo's $\mathrm{x}$ - (top left), $\mathrm{y}$ - (top right), and $\mathrm{z}$ - (bottom left) axis. The rough nature of the total field lines is due to a lower signal to noise ratio as compared to measurements taken at smaller source-sensor distances. 
The results from Figures 6.7, 6.8, and 6.9 indicate that the implementation of the CMC strategy leads to a significant reduction in the magnetic noise of the Hitec HSR5990TG servo. The percentage reduction was calculated for each data set (distance and rotation axis) to quantify the decrease in the magnetic signature. The percentage reduction was calculated as the mean absolute value of each magnetic field component for each distance and rotational axis:

$$
\% \text { reduction }=\frac{\frac{1}{n} \sum_{i=1}^{n}\left|\vec{B}_{i, \text { original }}\right|-\frac{1}{n} \sum_{i=1}^{n}\left|\vec{B}_{i, C M C}\right|}{\frac{1}{n} \sum_{i=1}^{n}\left|\vec{B}_{i, \text { original }}\right|} \times 100,
$$

where $\left|\vec{B}_{i, \text { original }}\right|$ and $\left|\vec{B}_{i, C M C}\right|$ are the absolute magnetic field values for vector field component $\vec{B}_{i}$, for the original and CMC source configurations, respectively, where $n$ is the number of measurement points per full rotation. A positive percentage reduction indicates a reduction in the magnetic signature of the servo with $\mathrm{CMC}$ as compared to the servo only, and vice versa.

Tables 6.1 to 6.3 compare the percentage reduction for the theoretical and experimental results for three distances. It can be noted that the percentage reduction in the magnetic signature of the Hitec HSR-5990TG increases with distance. This is due to the multipole effects - created by placing the cancellation magnet in close proximity to the servo - decreasing more rapidly with distance than the dipole effects. 
Table 6.1: Comparison of the percentage reduction in the magnetic signature of the Hitec HSR-5990TG servo as a result of utilizing the CMC strategy for measurements at a distance of $0.22 \mathrm{~m}$. The theoretical results (Theo) and experimental results (Exp) are provided. All units in \%.

\begin{tabular}{|c|c|c|c|c||c|}
\hline \multirow{2}{*}{ Rotational Axis } & \multirow{2}{*}{ Case } & \multicolumn{4}{|c|}{ Percentage Reduction [\%] } \\
\cline { 3 - 6 } & & $\vec{B}_{x}$ & $\vec{B}_{y}$ & $\vec{B}_{z}$ & $\vec{B}$ \\
\hline \hline \multirow{3}{*}{$x$ - axis } & Theo & 61.8 & 66.4 & 47.3 & 54.8 \\
\cline { 2 - 6 } & Exp & 68.1 & 62.1 & 54.2 & 60.5 \\
\hline \multirow{3}{*}{$z$ - axis } & Theo & -150.4 & 49.3 & 83.1 & 63.8 \\
\cline { 2 - 6 } & Exp & -171.8 & 76.1 & 76.9 & 57.5 \\
\hline & Theo & 48.7 & 46.8 & 61.9 & 51.2 \\
\cline { 2 - 6 } & Exp & 49.6 & 45.4 & 58.5 & 50.3 \\
\hline
\end{tabular}

Table 6.2: Comparison of the percentage reduction in the magnetic signature of the Hitec HSR-5990TG servo as a result of utilizing the CMC strategy for measurements at a distance of $0.33 \mathrm{~m}$. The theoretical results (Theo) and experimental results (Exp) are provided. All units in \%.

\begin{tabular}{|c|c|c|c|c||c|}
\hline \multirow{2}{*}{ Rotational Axis } & \multirow{2}{*}{ Case } & \multicolumn{4}{|c|}{ Percentage Reduction [\%] } \\
\cline { 3 - 6 } & & $\vec{B}_{x}$ & $\vec{B}_{y}$ & $\vec{B}_{z}$ & $\mid \vec{B}$ \\
\hline \hline \multirow{3}{*}{$x$-axis } & Theo & 54.1 & 76.2 & 75.9 & 68.5 \\
\cline { 2 - 6 } & Exp & 74.2 & 71.5 & 73.7 & 71.4 \\
\hline \multirow{3}{*}{$z$-axis } & Theo & 60.8 & -98.5 & 60.5 & 56.0 \\
\cline { 2 - 6 } & Exp & 55.4 & 69.8 & 82.0 & 69.3 \\
\hline & Theo & 57.2 & 55.2 & 65.7 & 61.3 \\
\cline { 2 - 6 } & Exp & 61.3 & 58.7 & 67.7 & 63.6 \\
\hline
\end{tabular}


Table 6.3: Comparison of the percentage reduction in the magnetic signature of the Hitec HSR-5990TG servo as a result of utilizing the CMC strategy for measurements at a distance of $0.52 \mathrm{~m}$. The theoretical results (Theo) and experimental results (Exp) are provided. All units in \%.

\begin{tabular}{|c|c|c|c|c||c|}
\hline \multirow{2}{*}{ Rotational Axis } & \multirow{2}{*}{ Case } & \multicolumn{4}{|c|}{ Percentage Reduction [\%] } \\
\cline { 3 - 6 } & & $\vec{B}_{x}$ & $\vec{B}_{y}$ & $\vec{B}_{z}$ & $\mid \vec{B}$ \\
\hline \hline \multirow{3}{*}{$x$-axis } & Theo & 39.8 & 86.6 & 85.4 & 77.9 \\
\cline { 2 - 6 } & Exp & 67.8 & 80.4 & 82.1 & 79.8 \\
\hline \multirow{3}{*}{$y$-axis } & Theo & 88.0 & -138.8 & 59.0 & 67.8 \\
\cline { 2 - 6 } & Exp & 79.7 & 23 & 73.1 & 74.4 \\
\hline \multirow{2}{*}{-axis } & Theo & 65.5 & 64.5 & 72.7 & 70.2 \\
\cline { 2 - 6 } & Exp & 72.9 & 71.4 & 76.0 & 74.5 \\
\hline
\end{tabular}




\subsection{Cancellation Conclusions}

The cancellation magnet configuration(CMC) strategy yielded an average improvement of $56.1 \%, 68.1 \%$, and $76.2 \%$ for all data points tested experimentally at distances of $0.22 \mathrm{~m}, 0.33 \mathrm{~m}$, and $0.52 \mathrm{~m}$, respectively. This constitutes a significant reduction in the magnetic noise contributions of single components. The CMC strategy could be easily implemented for all servo actuators onboard GeoSurv II. The CMC strategy is most beneficial for unpaired sources such as the nose landing gear and throttle servos. Paired servos, such as the flaperon, elevator, and rudder servos, may have their net magnetic signature reduced by counterorienting each servo relative to the other, along the dominant magnetic dipole axis.

The CMC approach requires the use of permanent magnets which are closely paired in magnetic dipole moment magnitude to sources. Care must be taken when orienting the cancellation magnet to each source since improper orientation may result in increased magnetic signature, which is undesirable.

Additional recommendations for this approach, are to investigate the use of multiple cancellation magnets to cancel multiple components of each source's magnetic dipole moment. Also, the manufacture of magnets of custom magnetization would allow for exact matching of the magnitude of each source's dipole. Furthermore, the effect of the cancellation magnet on servo performance was not investigated. It is possible that the cancellation magnet may reduce the performance of the servo. 


\section{Chapter 7}

\section{Testing and Analysis of Assembled GeoSurv II Prototype}

\subsection{Introduction}

Geomagnetic survey aircraft must fly a Figure of Merit (FOM) test at the beginning of each survey. A FOM involves manoeuvring the aircraft through different attitude variations (pitch, roll, yaw) in four flight directions (usually cardinal directions) to measure the magnetic noise variations for each heading and attitude combination. This allows for an evaluation of the magnetic signature of the aircraft at the survey location, and allows for partial compensation of the magnetic noise through data processing. Since an evaluation of the current magnetic noise of GeoSurv II was required to determine the level of improvements necessary to operate within design specifications, and because the GeoSurv II prototype has not achieved first flight at the time of this writing, an alternative testing scheme was required to develop a benchmark for the magnetic noise of the UAS while on the ground.

This chapter outlines testing of the assembled GeoSurv II prototype to determine magnetic noise at the wingtip magnetometers under varying conditions. For this series 
of testing, components that were known to be magnetic and are not intended to be used on the final GeoSurv II design were removed and replaced with nonmagnetic equivalents. These components include the steel ballast, the landing gear, and the wing-boom shear bolts, among others. As was stated in Section 1.2.2, the allowable magnetic noise is $\pm 0.1 \mathrm{nT}$ and $\pm 0.01 \mathrm{nT}$ for time invariant and time varying sources in a 0 to $5 \mathrm{~Hz}$ range, respectively. The test procedures, analysis, and results are described in greater detail in [29].

\subsection{Actuation of Control Surfaces}

Due to the proximity of the control surfaces to the onboard magnetometers, there was a concern that any magnetic materials within each control surface or control surface control systems may cause unwanted transient magnetic noise. All control surfaces on GeoSurv II are of nonmagnetic construction, but some of the control rods used to transfer forces from the servos to the control surfaces are known to be slightly ferromagnetic. Due to the difficulty of loading each control surface appropriately to simulate realistic flight behaviour, and since the servos will have been previously tested under loading, only the effect of moving each control surface on magnetic noise was studied for this investigation.

For this experiment, the GeoSurv II prototype was assembled in the Absolute Building of the Geomagnetic Laboratory. A Cesium magnetometer was placed at each respective wingtip magnetometer location. The UAS was wired and powered to allow proper actuation of all control surfaces using a radio controller. The experimental setup used can be seen in Figure 7.1.

Each control surface servo was tested by performing both fast and slow actuation. The slow actuation sequences comprised of roughly 3 cycles of minimum to maximum 


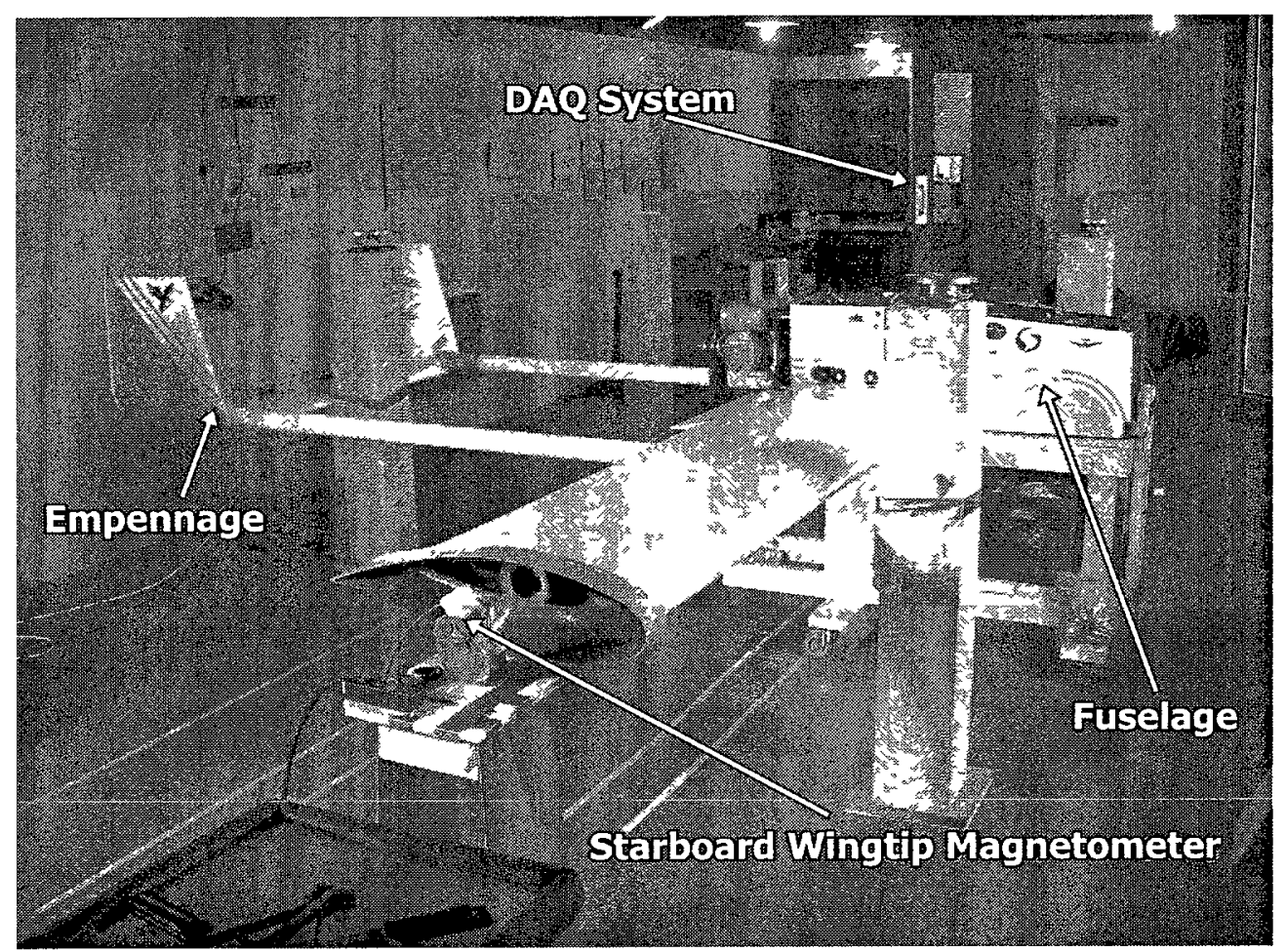

Figure 7.1: Experimental setup used for indoor full scale testing at the Geomagnetic Laboratory. Magnetometers were placed at the respective UAS wingtip locations.

travel points over approximately 25 seconds. The fast actuation sequences comprised of roughly 10 cycles of minimum to maximum travel points over approximately 10 seconds. The two speeds were taken to rule out any effect from a varying background field. Also, during baseline readings. the UAS was unpowered and all control surfaces were in the neutral position. A reference sensor was not used, so for each data set it was assumed that the background field did not vary throughout the experiment. (4.1) was used to generate the offset for each sensor.

As can be found in Appendix B, there does not appear to be any effect of actuating the elevator and rudder control surfaces, as well as for throttle actuation, on the transient magnetic noise experienced at the wingtips. Analysis of Figure 7.2 shows 
that actuating the flaperons affects the transient magnetic signature of the UAS. Additionally, the effect is greater at the starboard magnetometer than at the port magnetometer.
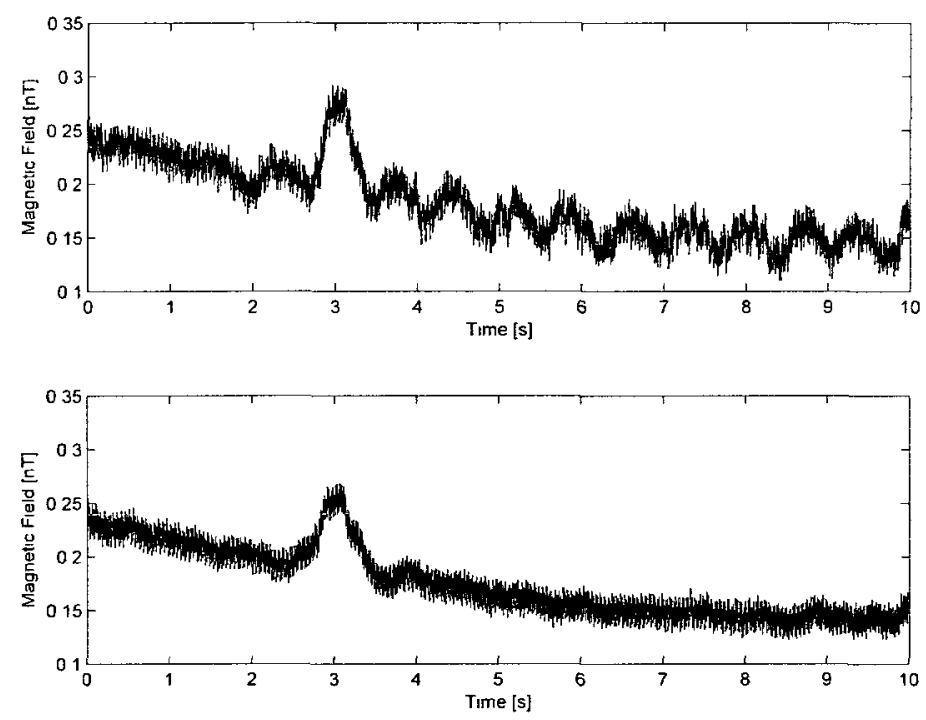

Figure 7.2: Behaviour of Magnetic Field while actuating both flaperons in a roll manocuvre using powered servos. The magnetic noise effect for the starboard (top) and port(bottom) magnetometers is shown for the fast actuation scenario. The magnetic noise observed at the wingtip magnetometers appears to be related to actuating the control surface, especially for the fast actuation sequence.

A FFT analysis was completed for the flaperon actuation data as can be seen in Figure 7.3. From the frequency domain analysis, it appears that the magnetic noise at the port magnetometer is within design specifications, but that the magnetic noise at the starboard magnetometer is slightly above design specifications for frequencies $\leq 1.5 \mathrm{~Hz}$. If the flaperon servos could be relocated to the fuselage this would almost certainly bring the transient behaviour to within the design specification of \pm 0.01 $\mathrm{nT}$ for time-varying noise in the 0 to $5 \mathrm{~Hz}$ bandwidth. 

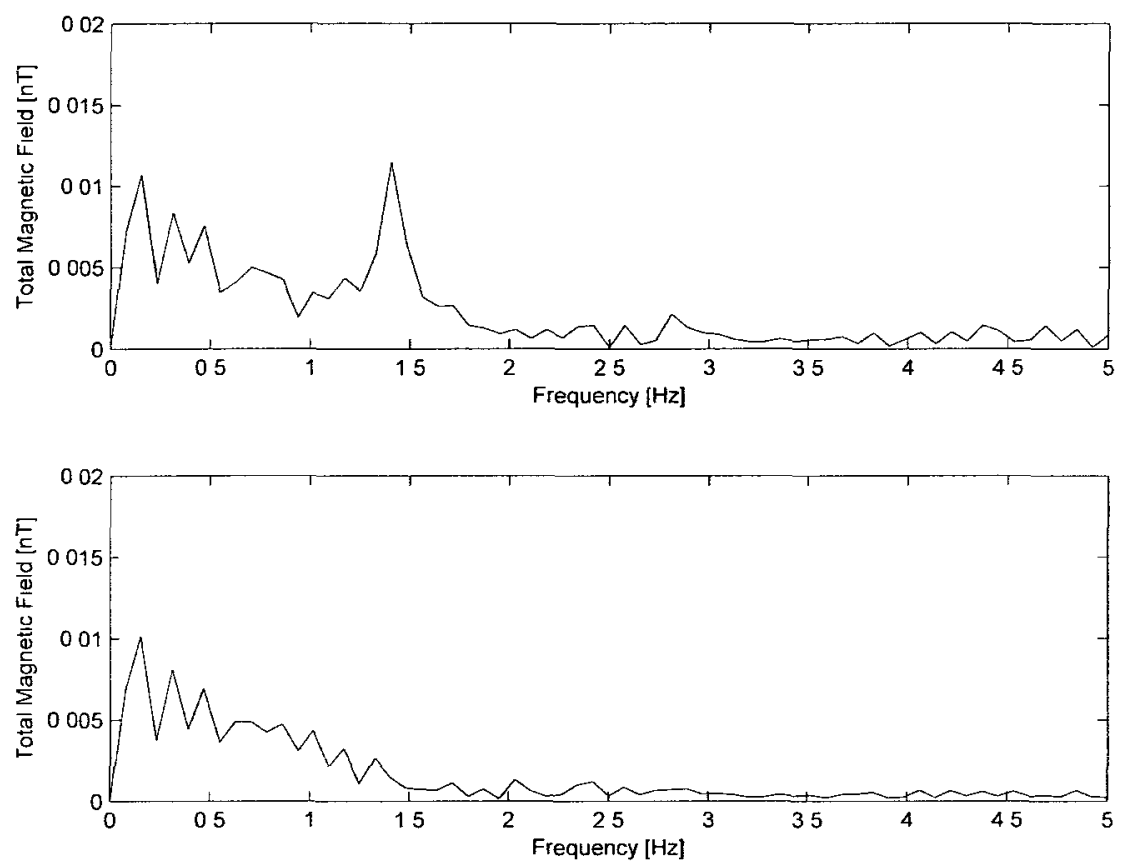

Figure 7.3: Behaviour of Magnetic Field while actuating both flaperons in a roll manoeuvre analyzed in the frequency domain. The transient magnetic noise at the starboard magnetometer appears to be above design specifications for frequencies $\leq 1.5 \mathrm{~Hz}$.

\subsection{Simulated Wingtip Deflection}

Since the wingtips are expected to deflect upwards under aerodynamic loading, it was necessary to determine the magnetic field properties above the wingtip sensor locations. Ideally, the variation of the magnetic field at the wingtip sensors will be negligible. For this test, the entire UAS remained stationary in the setup shown in Figure 7.1. with the wings experiencing no loads or deflections. Instead, the wingtip sensors were displaced vertically in incremental distances to simulate the effect of wingtip deflections. 
One wingtip was tested at a time, with the other wingtip magnetometer acting as a reference sensor. (4.1), (4.2), and (4.3) were used to remove any background field effects. Figure 7.4 indicates that the magnetic noise effect of wingtip deflection is much more severe on the starboard side than it is on the port side. However, a flaw in the experimental procedure was noted after testing was completed. When testing, the assumption was made that the background magnetic field did not vary in the upwards direction at either wingtip location, since a baseline reading was taken only at the undeflected state. This assumption may not be entirely valid, and as such it is recommended that when this experiment is conducted in the future that a baseline reading without the UAS present be taken to account for any local magnetic field gradients.

This series of testing may not be necessary in the future since there are a variety of ways in which the different sections of the UAS structure can move with respect to one another due to the airframe not being rigid. This makes the analysis of all possible deflections increasingly difficult. 


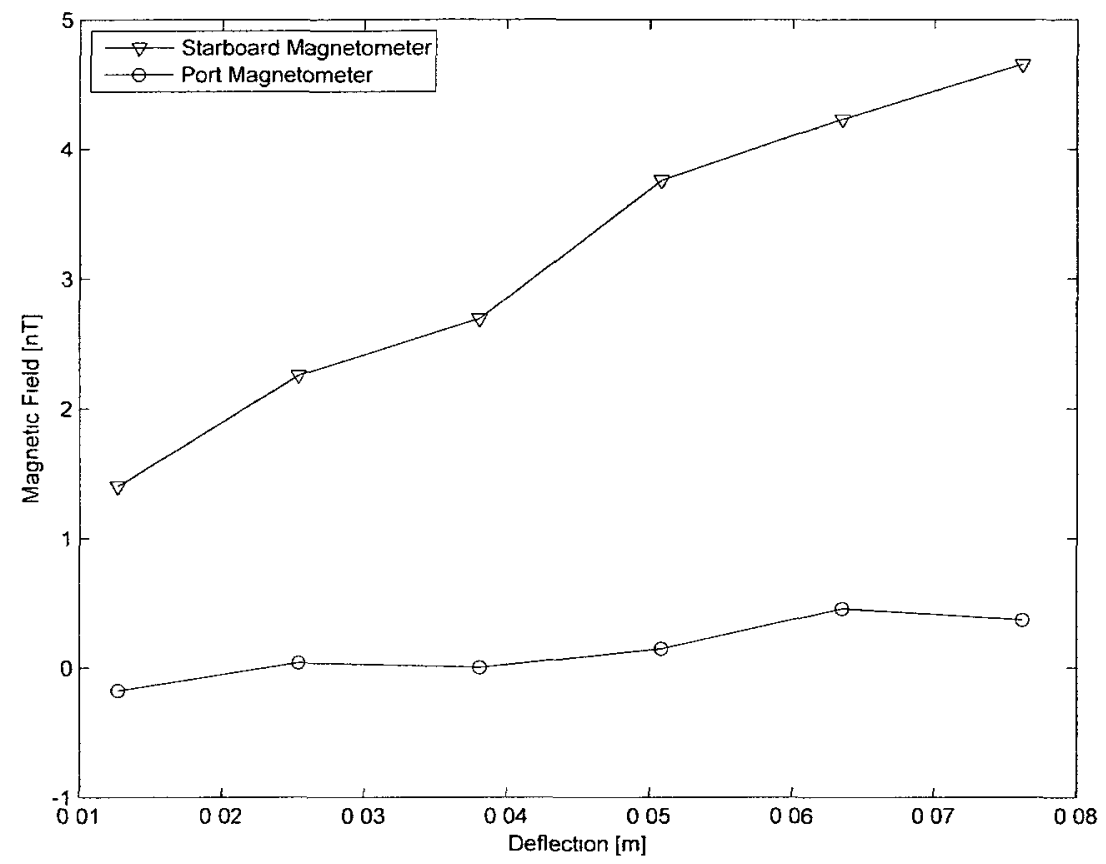

Figure 7.4: Behaviour of wingtip magnetic noise while simulating wingtip deflections. Results indicate that there is a large magnetic gradient above the starboard wingtip location. The gradient of the background field was not measured, so it is not known whether this effect is due entirely to the magnetic field produced by GeoSurv II.

\subsection{Gradiometer Test}

The concept behind this test is that by positioning the magnetometers in a gradiometer configuration, it is possible to determine the locations at which the magnetic noise is greatest for sensors spaced according to the current GeoSurv II design. The procedure of this experiment follows the gradiometer procedure outlined in Section 4.2.3. The magnetometers were spaced to allow the inner wingtip fairing to pass on either side with approximately $0.05-0.08 \mathrm{~m}$ of clearance. A picture taken during testing is shown in Figure 7.5. 


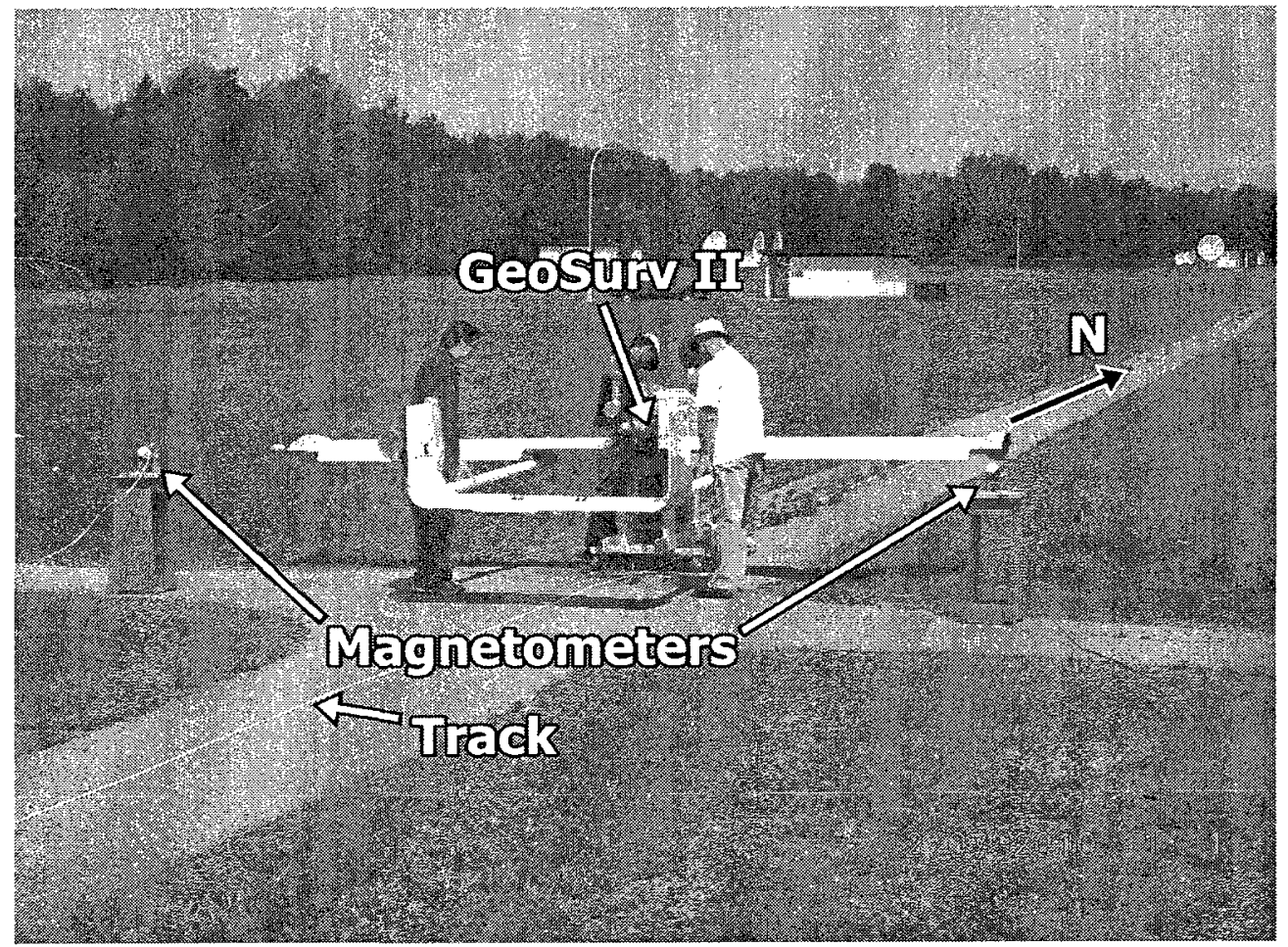

Figure 7.5: Experimental setup used for full scale gradiometer testing at the Geomagnetic Laboratory. GeoSurv II was moved in incremental distances manually past two stationary Cesium magnetometers in a gradiometer configuration by nonmagnetic volunteers in a South-North direction.

Baseline readings were taken before and after each data set, and the measurement values were calculated using (4.1), (4.2), and (4.4). From Figure 7.6, it can be observed that the UAS has several locations at which the magnetic noise peaks indicating multiple sources of magnetic noise. It is also interesting to note that the magnetic noise experienced at both wingtips was not the same, meaning that the total magnetic noise of all sources is not symmetric about the longitudinal axis of the UAS. 


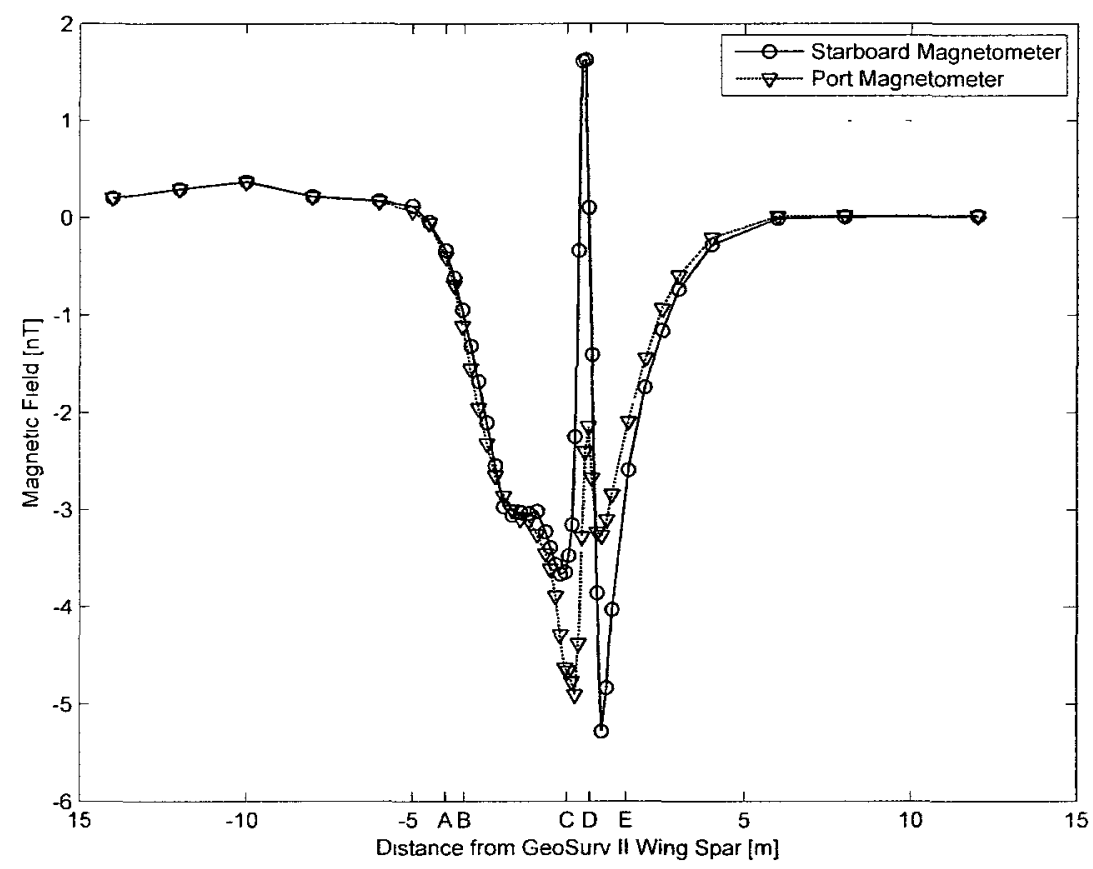

Figure 7.6: Behaviour of magnetic noise for GeoSurv II while moving past two Cesium Magnetometers in a gradiometer configuration. Axis reference labels: (A) empennage trailing edge, (B) empennage leading edge, (C) wing trailing edge, (D) wing leading edge, and (E) nose. The magnetic noise is largest near the wings, a result that is unfavourable for the current GeoSurv II design.

Additionally, Figure 7.7 indicates that the largest magnetic noise gradient between the two sensors occurs at the wingtip location. This results is undesirable since this is where magnetometers are designed to be located on the GeoSurv II design. As explained in Chapter 5, this was determined to be due to the orientation of the magnetic dipole within each servo and the combined effect due to the configuration of the empennage servos. Since all paired servos are similarly oriented, this results in the large magnetic noise exhibited on the GeoSurv II prototype. 


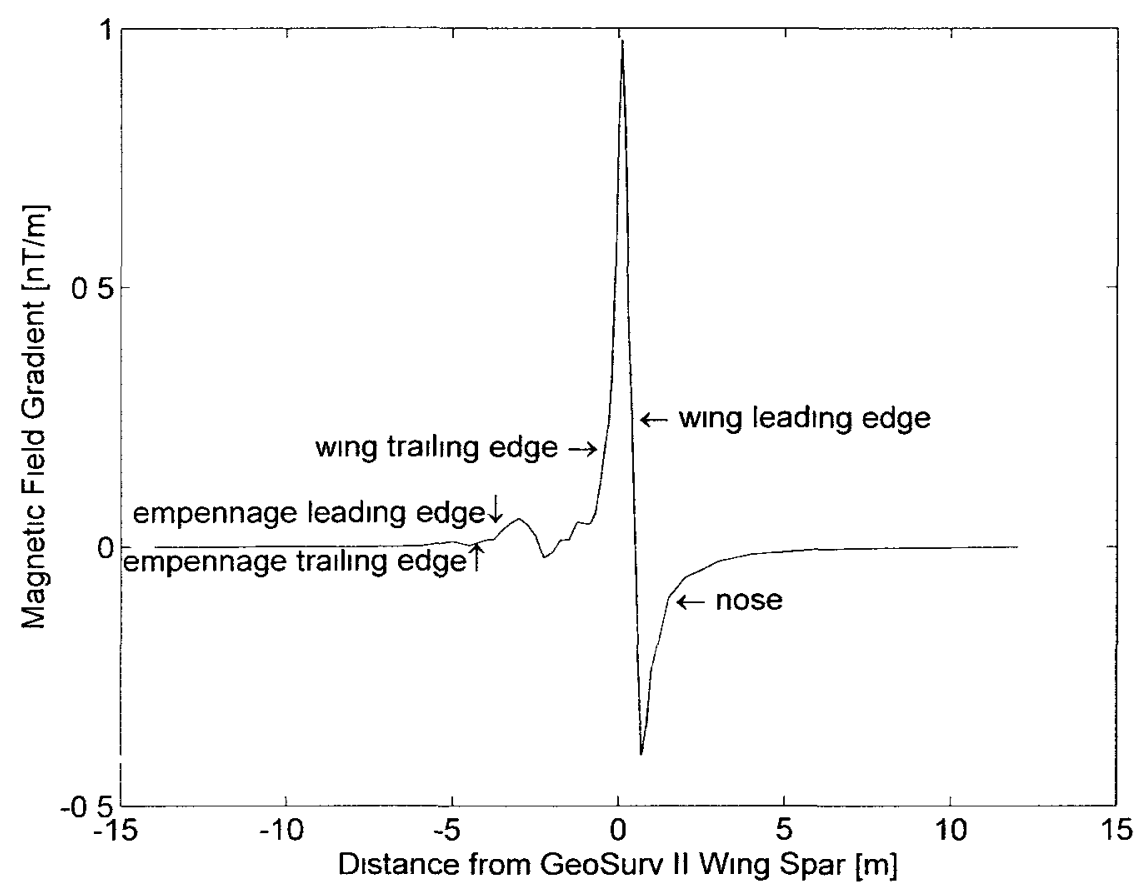

Figure 7.7: Behaviour of magnetic noise gradient for GeoSurv II while moving past two Cesium Magnetometers in a gradiometer configuration. The largest magnetic field gradient occurs at the wingtip locations.

\subsection{Heading Test}

This test provided the most definitive measure of the magnetic signature of GeoSurv II without actually performing a Figure of Merit (FOM) described in Section 7.1. The magnetic noise experienced at the wingtip sensors was expected to vary with the heading of the UAS due to the interaction of Earth's background magnetic field with the magnetic noise sources onboard GeoSurv II.

GeoSurv II was rotated about the vertical axis intersecting the centre of the wing carry-through spar through varying headings. The locations of measurements are shown in Figure 7.8, and the experimental setup is shown in Figure 7.9. It is worth noting the location of testing was fairly flat such that the experiment effectively 
simulated level flight at different headings

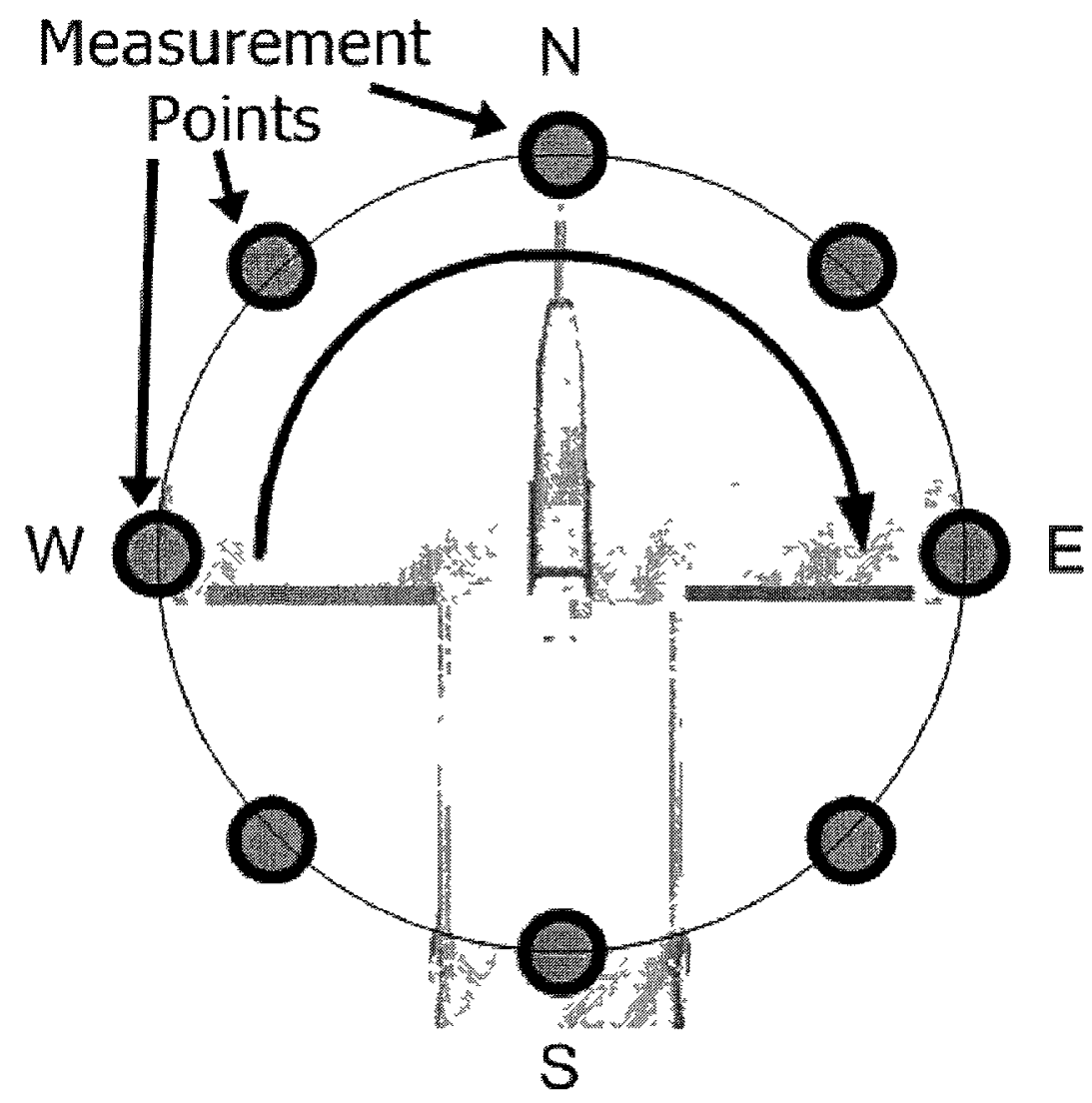

Figure 7.8: Locations of measurement points used during full scale heading test. Baseline readings were taken at each of the measurement points to allow for the background field to be removed from test results. Magnetic noise measurements were taken for each wingtip at headings of North, Northeast, East, Southeast, South, Southwest, West, and Northwest, which correspond to the measurement points shown. 


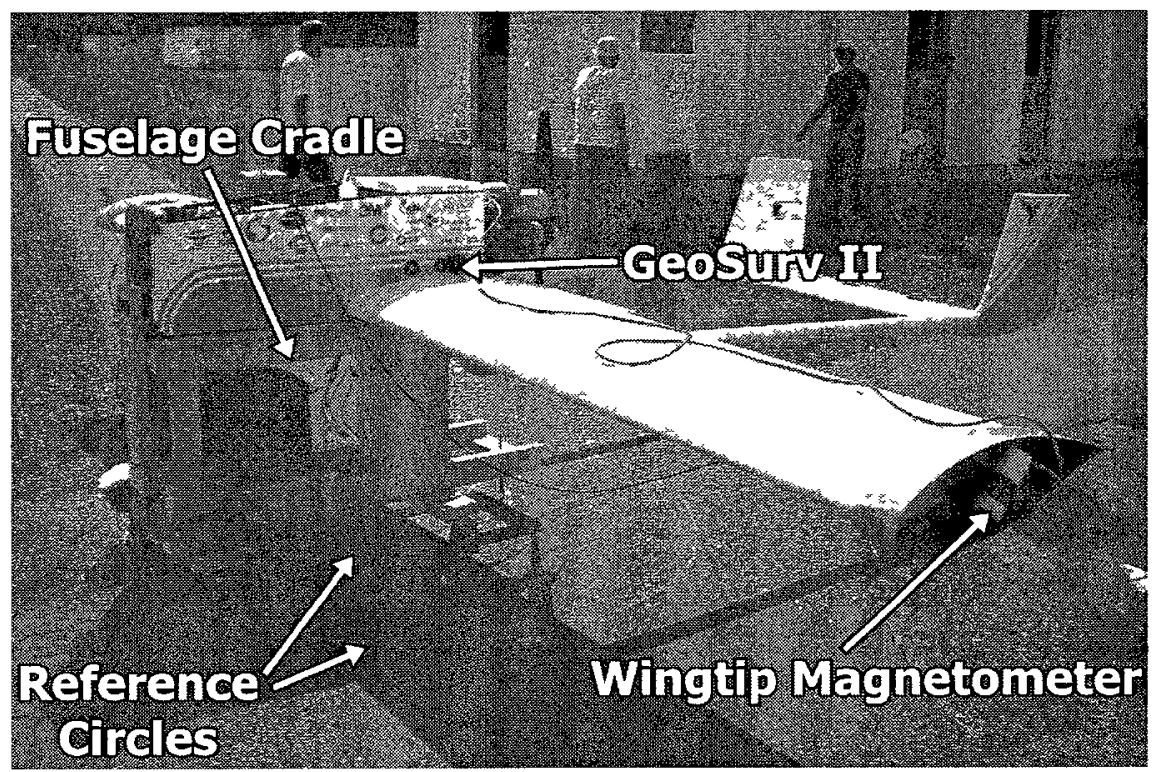

Figure 7.9: Experimental setup used during full scale heading test. The magnetic noise was evaluated one wingtip at a time. A reference magnetometer was used to measure changes in the background magnetic field. The maximum local magnetic field gradient was $2.64 \mathrm{nT} / \mathrm{m}$.

The background field was removed using baseline readings taken at locations corresponding to the wingtip positions at various headings; these baseline readings were taken with GeoSurv II positioned far enough away to not affect the local magnetic field of the test area. Since a reference sensor was used, any variations in the background magnetic field were accounted for using (4.2) and (4.3). It can be seen in Figure 7.10 that the magnetic noise is more than two orders of magnitude larger than the allowable magnetic noise as defined by the design specifications [3] as $\pm 0.1 \mathrm{nT}$ for time invariant sources. Additionallv, there is a significant difference between the magnetic noise behaviour at the starboard and port wingtip locations, meaning that additional measures will be needed to further reduce the magnetic signature. It may also be noted that largest total noise occurs when GeoSurv II is in a North-East heading, and the smallest total noise occurs when GeoSurv II is in a South-West heading, implying that survey lines should be flown at a South-West heading, which is not 
practical from a geomagnetic surveying standpoint.

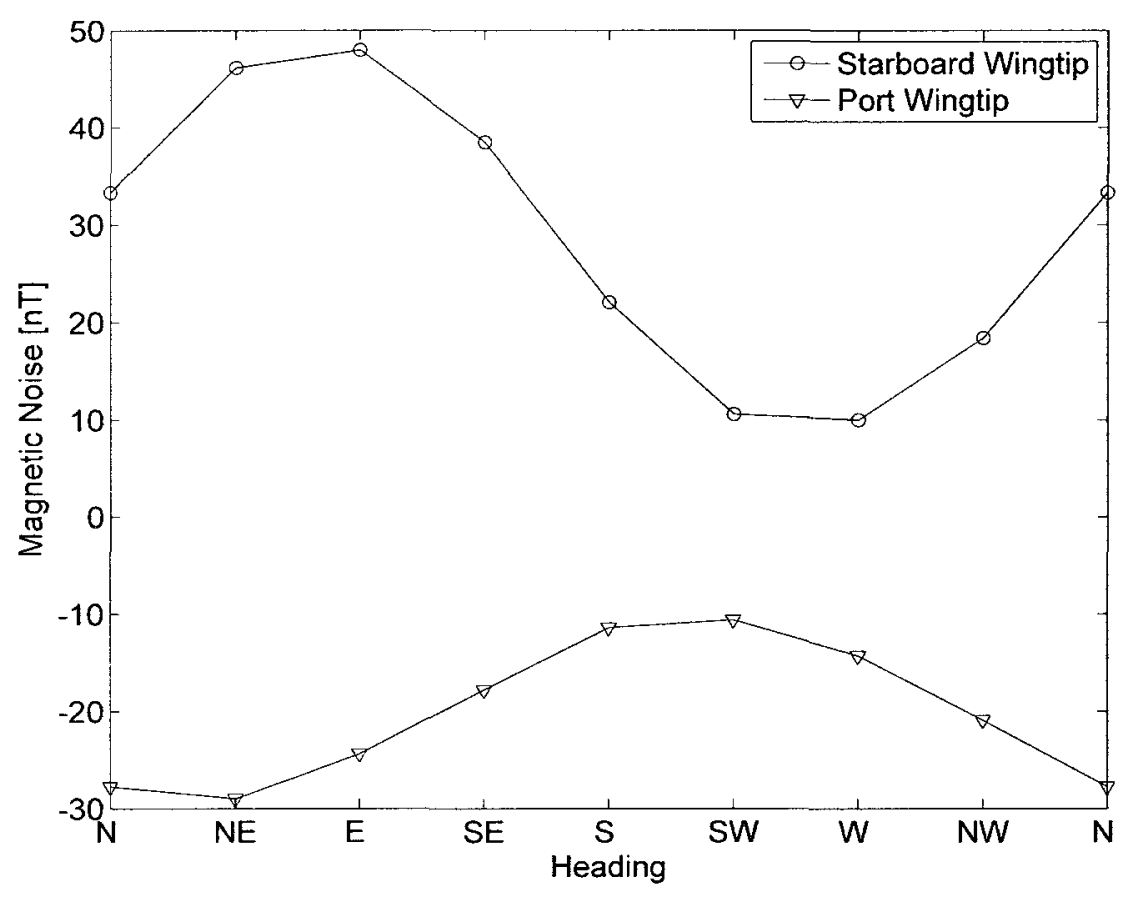

Figure 7.10: Behaviour of wingtip magnetic noise for GeoSurv II at various headings. The measured wingtip magnetic noise is larger than the allowable magnetic noise, and is significantly different for both wingtips.

It may be noted that when comparing the magnetic noise values for a North heading from Figure 7.10, $33.3 \mathrm{nT}$ (starboard) and $-27.7 \mathrm{nT}$ (port), to those from the gradiometer testing corresponding to the measurement where the the wingtips were closest to the magnetometers, $-0.3 \mathrm{nT}$ (starboard) and $-4.4 \mathrm{nT}$ (port), that the values do not agree. Note that the values from the gradiometer testing were taken approximately $0.15 \mathrm{~m}$ further away from the centreline of the UAS. The reason for this difference was not determined during the course of this research. It is believed that there is a large magnetic field gradient near the wingtip locations which may have led to the difference in values. 
Additionally. the difference in the magnetic noise behaviour at both wingtips was determined to be due to asymmetry of the magnetic field sources. Even though the magnetic field sources on GeoSurv II are geometrically symmetrical about the longitudinal axis of the UAS, the magnetic dipole moment within each servo is not aligned with the geometry of the servo, leading to an unsymmetrical magnetic profile.

\subsection{Full-scale Testing Conclusions}

From the full scale testing, it may be concluded that the wingtip magnetic noise of the current GeoSurv II prototype does not meet design specifications. The transient noise due to the actuation of the flaperon control surfaces may be reduced by increasing the distance between flaperon servos and magnetometer locations. Additionally, the magnetic noise found at the wingtip locations highlights the requirement for the use of additional methods to reduce wingtip magnetic noise. These methods may include cancellation schemes, and source configuration optimization schemes designed to reduce magnetic noise at wingtip magnetometer locations. 


\section{Chapter 8}

\section{Genetic Algorithm Magnetic Signature}

\section{Optimization}

\subsection{Introduction}

After analysis of the magnetic signature of GeoSurv II as described in Chapter 7, it was concluded that it would be necessary to use multiple approaches to minimize the wingtip magnetic noise. The first approach being to minimize each source locally through cancellation. The second approach being to configure each source such that the combined effect at the wingtip magnetometer locations would be minimized.

The Genetic Algorithm Magnetic Signature Optimization (GAMSO) strategy was designed to optimize the configuration (location and orientation) of magnetic noise sources such that the combined effect at each wingtip magnetometer location was attenuated, equalized, and spatially uniform. This strategy was developed using custom MATLAB scripts, which are completely modular allowing for easy and simple modification of parameters such as source bounds and sensor measurement points. The GAMSO package presented here was created for the GeoSurv II UAS prototype, but can easily be modified for other applications. 
This chapter will present a brief summary of genetic algorithm theory, outlining the key components of genetic algorithms, along with alternative implementation strategies for each component. Additionally, a detailed description of how the genetic algorithm was applied to GeoSurv II magnetic source configuration through the development of GAMSO will be provided. The effectiveness of GAMSO to determine the optimal solution is evaluated through an analysis detailed in this chapter. Finally, theoretical results from the GAMSO strategy are presented and compared to experimental results.

\subsection{Genetic Algorithm Theory and GAMSO Im- plementation}

Genetic algorithms come from a branch of evolutionary algorithms that locate a population of optimal solutions through a stochastic heuristic search. Initial work on the subject was presented by Holland [33], and further expanded to a variety of practical problems by Goldberg [34]. Genetic algorithms operate by subjecting a population of possible solutions to the biological processes of genetics and evolution. Haupt and Werner [35] state that "genetics is the study of the inheritance and variation of biological traits" and evolution "results in genetic changes through natural selection, genetic drift, mutation, and migration." Individuals within populations are evaluated on their fitness, and subjected to evolutionary forces which yield a population of individuals that are best suited within a defined environment. In terms of optimization, the fitness of each individual is evaluated by a defined cost or objective function. It is the fitness function that defines the environment.

Traits of successful individuals may be carried directly over to the next generation, known as elite selection (natural selection in the biological world), or may be mixed 
with the traits of other successful individuals in a process created to mimic mating called crossover. In order to maintain genetic diversity, individuals are also subject to mutation whereby one part of their genome is altered to produce a new individual. Each new population generated is referred to as a generation. The genetic algorithm may be terminated if a set number of generations is reached. the best fitness function of the population has converged. or if a defined amount of time has elapsed, among other options. A detailed explanation of how these evolutionary processes are implemented and integrated into GAMSO will be explained in this section.

The general process of genetic algorithms begins with creating an initial population, for which the fitness function of each individual in that population is evaluated. Individuals are then selected for elite selection and crossover based on their fitness function. Additionally, individuals are selected, usually at random, for mutation. The subpopulations created by elite selection, crossover, and mutation are then combined to create the population defining the next generation. Before evaluating the fitness function of the individuals in the next generation, termination conditions are evaluated to ensure that subsequent generations are necessary. The process followed to implement genetic algorithms is shown in Figure 8.1.

The advantages of genetic algorithms over other optimization strategies are as follows [35]:

- Ability to handle both continuous and/or discontinuous variables.

- No need to supply or to calculate any derivative information.

- Ability to handle a large number of variables over complex cost surfaces.

- Output generates a list (population) of optimum solutions, instead of a single solution. 


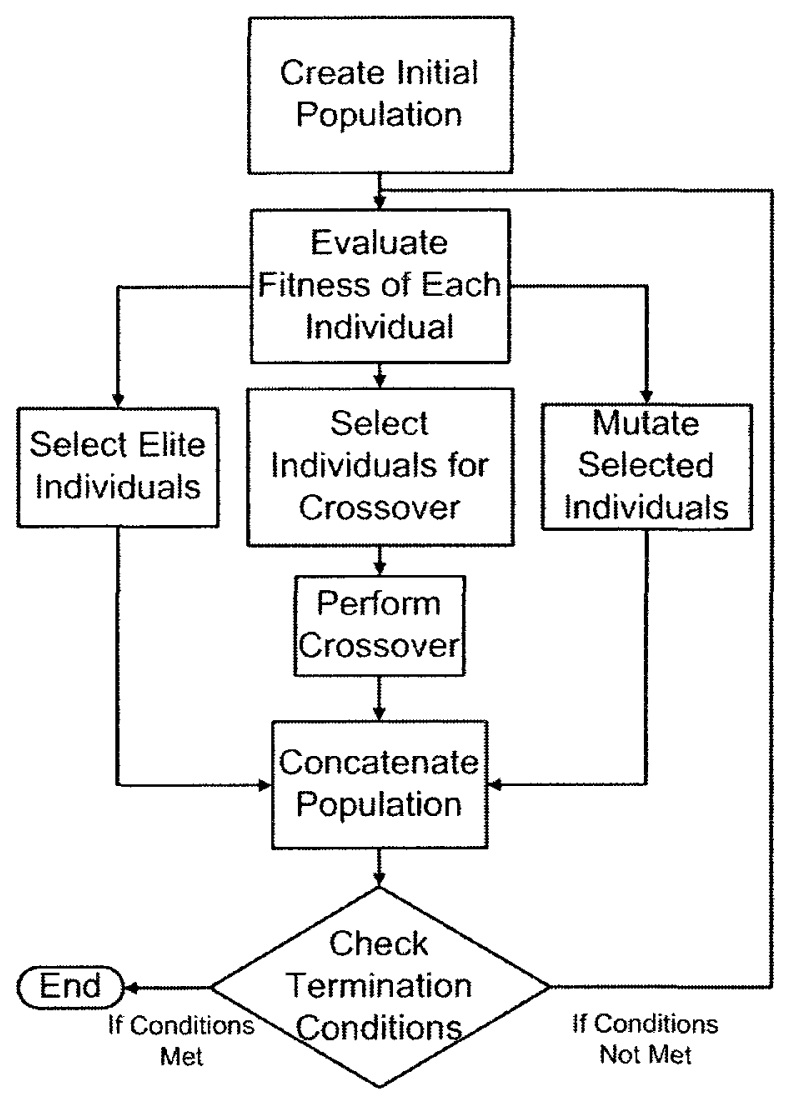

Figure 8.1: Block diagram of the processes used in genetic algorithms. The main processes are creation, selection, mutation, and crossover, which all allow the population to change from one generation to the next.

- Relatively easy to implement and to alter once created.

Some disadvantages of genetic algorithms compared to other optimization strategies are as follows [35]:

- Fast initial convergence, followed by slow improvements as the genetic diversity of the population decreases. Genetic algorithms are often paired with other optimization algorithms for this reason.

- Uncertainty of termination conditions for some problems. It is not clear when the global optimum has been reached. 
- Tendency to converge to local optima, rather than the global optimum. There are algorithms which may be built in to avoid this such as imposing a niche penalty.

\subsubsection{Constraints}

Genetic algorithms are able to handle many types of variables and constraints, including continuous and discontinuous variables. Variables in genetic algorithms are known as genes. Variables used in genetic algorithms must be numerical, although nonnumerical values can be used as long as they are assigned a numerical value. A sequence of genes is known as a chromosome. The chromosome is the entire string of genes which define an individual. Furthermore, a collection of chromosomes is referred to as a population. It is possible to have identical individuals within the same population.

Individuals can be evaluated against defined constraints, and individuals with constraint violations may be treated in several different ways, A few of the possibilities are listed below:

1. Individuals can be assigned an arbitrarily large fitness function value. This will ensure that the individual is not selected as part of the elite population, and will significantly decrease the probability of the individual being chosen as a parent for crossover. However, this individual may still be selected for mutation in some schemes which may not necessarily correct the constraint violation.

2. A new individual may be generated using the creation algorithm, taking the place of the individual with the constraint violation. This does not guarantee that the replacement individual will not have have a constraint violation. 
3. Application of a penalty term to the fitness function of the individual such that the probability of the individual being selected for crossover is decreased but not to the same extent as Option 1. This individual may still be selected for mutation.

4. The individual may be repaired using a repair heuristic as outlined by SalcedoSanz [36]. The genes which cause the constraint violation may be identified and altered such that the individual no longer violates the defined constraints.

Options 1 to 3 are easy to implement, but may involve the premature removal of that individual's genes from the population which may cause slower convergence towards the optimal solution. Option \& involves added complexity to the algorithm, but will often yield a faster convergence towards the optimal solution [36]. Option 1 was chosen for constraint handling in GAMSO due to its ease of implementation. The slower convergence of this option was deemed acceptable since this is an entirely offline application.

Upper and lower bounds may be established for each gene. Provided that the upper and lower bounds are respected in the creation and mutation functions, they will not be violated in the remainder of the algorithm.

The configuration of each source may be defined by six variables which are $\mathrm{x}-, \mathrm{y}^{-}, \mathrm{z}-$ locations and rotations about the $\mathrm{x}-, \mathrm{y}-$, and $\mathrm{z}$-axis. Since the GeoSurv II prototype uses 14 servos as shown in Figure 1.3, this would yield a problem with a total of 84 variables/genes. The total number of variables may be further reduced with the assumption that sources are configured symmetrically about the xz-plane of the UAS (reference Figure 8.2). This means that by defining the starboard flaperon, elevator, and rudder servo pairs, that the configuration of their port counterparts may be 
defined through a simple translation and rotation of the starboard equivalents. This reduces the total number of variables/genes to 48 . The constraints applied to each GAMSO gene result in a chromosome that is composed of 24 continuous variables (locations), and 24 discontinuous variables (rotations).

Upper and lower bounds for the location of each source were defined as follows:

- Control Surface (flaperon, elevator, rudder) Servos: Sources may be located anywhere along the span of each control surface, and must be contained by the chord and camber of the airfoil corresponding to the control surface.

- Throttle Servo: Source may be located anywhere in the standoff gap between the rear of the fuselage and the engine mounting plate.

- Nose Landing Gear (NLG) Servo: Fully constrained location due to the geometry of the NLG. The location was defined as a variable rather than a constant to allow the option of location optimization for future versions of GeoSurv II that may include a redesigned NLG.

The bounds for both location and orientation are defined in the script GAMSObounds.m. Additionally, the locations of the control surface servos were constrained such that they would not interfere geometrically with one another. This was implemented by ensuring that the minimum centre-to-centre distance for each servo pair was greater than a defined offset, which in the case of the Hitec HSR-5990TG is 0.065 m. A separate function named GAMSOconstraint.m is called to determine if there are any constraint violations. Constraint violation repair was completed in the main script GAMSOmaster.m.

Finally, the orientation of each source was constrained such that it must be orthogonal to the coordinate planes of the UAS, defined in Figure 8.2. This constraint was 
introduced for two reasons. The first being that it is much simpler to install servos that are orthogonal to the coordinate planes. More importantly, this was necessary to ensure that the mechanical force from each servo could be utılized easily with a simple control linkage.

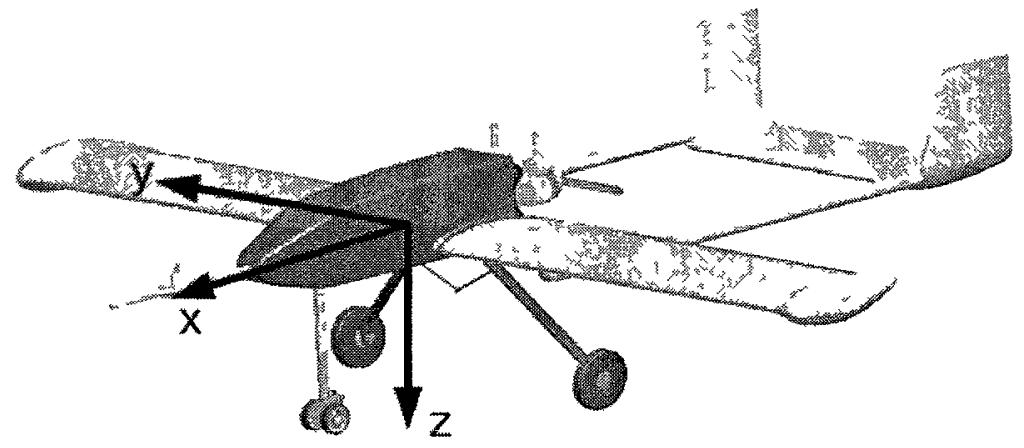

Figure 8.2: GeoSurv II coordmate system used for GAMSO strategy. $x$ is along the longitudinal axis, $y$ is along the lateral axis, and $z$ is along the vertical axis. Origin is at the intersection of the wing carry-through spar and lateral centre of the fuselage.

Additionally, for the control surface servos, a further subset of the orthogonal orientations was utrlized to ensure that the plane of travel of each servo armature contained the $\mathrm{x}$-axis of the UAS in order for the servo torque to be utilized directly. In other words, the plane of travel of the servo armature must not be in the yz-plane of the UAS. A list of feasible servo orientations was generated using the script $G A M$ SOconfigs $m$ which is used for creation and mutation of orientations. Also defined in GAMSOconfigs.m are two lists of orientations corresponding to the two separate rotation matrices needed to properly generate port sources from defined starboard sources. 


\subsubsection{Fitness Function}

In biology, the fitness of an individual is related to its ability to survive, and reproduce within its environment. In genetic algorithms, the fitness of an individual is evaluated using a fitness function which is akin to cost or objective functions in optimization. The objective of genetic algorithms is to minimize the fitness function value of the population. The most fit individual in a population will be the one with the lowest fitness function value. The fitness function determines the suitability of an individual for elite selection and crossover. The genes composing each individual's chromosome are more probable to be carried over to the next generation if the individual has a favourable fitness within the population.

The fitness function in GAMSO was designed to attenuate, equalize, and make spatially uniform the magnetic flux density at the wingtip magnetometer locations. Due to aerodynamic loading, there will be deflection of the wingtips during flight, resulting in translation of the wingtip magnetometers relative to the rest of the airframe. Additionally, the location of the magnetometer sensors may be anywhere within a $0.178 \mathrm{~m}$ (7") diameter sphere within the wingtip fairings, as defined by SGL. For this reason, a cubic array of measurement points at each wingtip magnetometer location was created to represent the $0.178 \mathrm{~m}$ diameter sphere, and to allow for wingtip deflections of up to $0.089 \mathrm{~m}$. Each measurement point within each magnetometer domain is also assigned a weight proportional to its proximity to the centre of the respective magnetometer domain. This was used to assign higher priority for fitness to the points at the centre of each magnetometer domain than to points near the exterior of the domain. The weighting of each measurement point, $W$, is calculated as follows

$$
W=\frac{1}{\|\vec{r}\|},
$$


where $\|\vec{r}\|$ is the distance from the centre of the magnetometer domain to the measurement point. Since a measurement point is defined at the centre of the magnetometer domain, and since the associated weighting factor would be infinite, a separate weighting factor is assigned to this measurement point with the following equation:

$$
\|\vec{r}\|_{c e n t r e}=\frac{D_{m a g}}{\sqrt[3]{n^{2}}-\sqrt[3]{n}}
$$

where $D_{\text {mag }}$ is the diameter of the magnetometer domain, which in the case of GeoSurv II is $0.178 \mathrm{~m}$, and $n$ is the number of measurement points. (8.2) simply calculates the distance between measurement points divided by the number of measurement points along each side of the cubic array of measurements. This weighting factor is used to calculate the attenuation and equalization. In GAMSO, the magnetometer measurement points along with their respective weighting factors were generated in a scripted called genmagpoints. $m$.

The three fitness metrics are explained below:

\section{Attenuation}

Attenuation refers to the decrease in magnetic flux density in regions of interest. Since the magnetic field at a point is the vector sum of all of the contributions from individual sources, it is possible to create small regions where the magnetic field is significantly decreased. This is the most important metric for the GeoSurv II application and as such is the most heavily weighted.

The equation used to evaluate the attenuation within each magnetometer domain is expressed as:

$$
\text { Attenuation }=K_{\text {atten }} \frac{1}{n} \sum_{i=1}^{n} W_{i}\left\|\vec{B}_{i}\right\|,
$$


where $K_{\text {atten }}$ is the penalty term used to ensure that the attenuation remains the most heavily weighted part of the fitness function, and $\vec{B}_{\imath}$ is the magnetic field at point $i$. This calculation must be completed for both magnetometer domains separately in order to make sure that both are attenuated.

\section{Equalization}

Equalization refers to the difference in magnetic field between the starboard and port magnetometers. This metric is important because the magnetic ficld gradient measured in $n T / m$ is often used in the analysis of geomagnetic data. If the difference in the magnetic noise between the starboard and port magnetometer locations is too large, then errors are introduced into the analysis of the magnetic field gradient.

When calculating the equalization. it is necessary to consider the difference in the vector field between starboard and port magnetometers. This is necessary because the interference vector which is the vector sum of the individual contributions of all individual's will also combine with the background field vector. If the port and starboard interference vectors are not oriented identically, they will interact with the background magnetic field to produce a different amount of magnetic noise at each wingtip even though they may have identical magnitudes in the zero background field scenario. This point can be visualized by referring to Figure 5.2.

The formula used to calculate the equalization in GAMSO is as follows:

$$
\text { Equalization }=K_{e q} \frac{1}{n} \sum_{\imath=1}^{n} W_{\imath}\left\|\vec{B}_{e q, \imath}\right\| \text {, }
$$

where,

$$
\overrightarrow{B_{e q, \imath}}=\vec{B}_{\text {starboard }, \imath}-\vec{B}_{\text {port }, \imath}
$$


where $\vec{B}_{\text {starboard,i }}$ and $\vec{B}_{\text {port }, \imath}$ are the vectors of magnetic interference for measurement. point $i$, and $K_{e q}$ is the penalty term for the equalization metric. The weighting factor, $W$, is included in (8.4) since it is more probable that the magnetometer will be at the centre than at the extremes of each domain, making it more desirable to equalize the points closest to the centre than it is for points further from the centre.

It should be noted that it is necessary to compare corresponding points. For example, the point that is lowest, furthest aft, and closest to the fuselage on the starboard side must be compared to the corresponding point on the port side. In GAMSO, this was completed by arranging the order of the magnetometer measurement points such that corresponding points are in the same order for both magnetometer domains.

\section{Spatial Uniformity}

Spatial Uniformity refers to attempting to make the magnetic noise gradient within each magnetometer domain as small as possible. This is necessary since the location of the magnetometer within the domain will not be constant during flight. Due to aerodynamic loading, there will be varying amounts of wingtip deflection causing the location of the magnetometer to change with respect to the rest of the UAS. If the magnetic field gradient within the magnetometer domain is too great, then this will appear as time-varying magnetic noise as the wingtip deflection changes throughout the mission.

The uniformity of the magnetic field within each domain may be measured by simply finding the difference between the maximum and minimum magnetic field within each domain. This calculation is shown below:

$$
\text { Uniformity }=K_{u n}\left(\|\vec{B}\|_{\max }-\|\vec{B}\|_{\min }\right)
$$


where $K_{u n}$ is the penalty term for spatial uniformity; $\|\vec{B}\|_{\max }$ and $\|\vec{B}\|_{\text {min }}$ are the maximum and minimum values of the total magnetic field within a specific magnetometer domain, respectively. The spatial uniformity must be calculated for both magnetometer domains separately.

\section{GAMSO Fitness Function}

Since fitness must be represented by a scalar, it is nccessary to combine all of the fitness metrics into a single value. In GAMSO this was performed by the summation of all fitness metrics, as shown below:

$$
\begin{aligned}
\text { Fitness }= & \text { Attenuation }_{\text {starboard }}+\text { Attenuation }_{\text {port }} \\
& + \text { Equalization }+ \text { Uniformity } \text { starboard }_{\text {for }}+\text { Uniformity }_{\text {port }}
\end{aligned}
$$

Additionally. it was necessary to define the penalty terms $K_{a t t e n}, K_{e q}$, and $K_{u n}$ through experimentation with GAMSO. These values were chosen such that the attenuation components had the greatest contribution, followed by roughly equal weighting for the equalization and uniformity components.

In GAMSO, the fitness function is calculated using the GAMSOmagfield.m script. This script outputs the magnetic field vector and total field at each measurement point, along with the fitness function components and fitness function sum. Only the fitness function sum is used during the genetic algorithm. The magnetic vector and total field measurements, and fitness function components are used in post-processing.

\subsubsection{Population Creation}

The first step in a genetic algorithm is to define the initial population. The initial population controls the genetic diversity of the population used in the first generation 
of genetic algorithm. The population of a generation is defined by a matrix that is of size Total Population $\times$ Number of Genes. Each row in the population matrix represents the chromosome of an individual. The initial population may be defined in one of three ways:

1. Generate New Population: Initial population may be randomly or uniformly generated using a creation function. The creation function may be hidden as is often the case in genetic algorithm toolboxes, or it may be customized as is the case with custom coded genetic algorithms. When initiating a genetic algorithm for the first time, it is necessary to generate a new population. Each individual in this population is a guess at the optimum solution.

2. Previous Final Population: The initial population may also be defined as the final population of a previous run of the genetic algorithm. This means that the genetic algorithm may be repeatably run for a discrete number of generations, whereby the final population of one run becomes the initial population of the next such that the total number of generations is equal to the sum of the number of generations from the individual runs. It is still necessary to initialize the genetic algorithm with a generated population for the first generation.

3. Combination: An initial population may also be defined as a combination of randomly or uniformly generated individuals and individuals from a previous run. This is useful for the scenario when a population is to be expanded from one run to the next. The final population of the previous run can be imported into the current run, and can be supplemented with a generated population to accommodate an increase in total population size.

The GAMSO strategy is able to utilize all three listed options. The ability to run a consecutive series of genetic algorithm runs, as explained in Method 2, was quite 
useful in terms of saving populations throughout the optimization. This allowed optimization over a large number of generations to be completed in a series of smaller runs.

In GAMSO, to define a new population use the GAMSOcreation.m script which generates a random population of potential solutions. It creates the locations and orientation portions of the population separately since the locations are continuous and the orientations are discontinuous. For sources that must be oriented in one of a subset of feasible orientations, an orientation previously defined by GAMSOconfigs. $m$ is randomly selected. Additionally, the current configuration of GeoSurv II and the manually optimized configuration (servo pairings have $+y$-axis of one servo counteroriented with respect to the other but positions remain the same) were seeded into the initial population. A brief description of each configuration is provided in Table 8.1 for reference.

Table 8.1: Brief description of configurations used during optimization.

\begin{tabular}{|c|l|}
\hline Configuration & Description \\
\hline Current & $\begin{array}{l}\text { Servo locations and orientations same as } \\
\text { on current GeoSurv II prototype. }\end{array}$ \\
\hline Manually Optimized & $\begin{array}{l}\text { Servo locations same as on the current } \\
\text { GeoSurv II prototype. Servo pairs (flap- } \\
\text { eron, elevator, rudder) are counterori- } \\
\text { ented such that their x-axes are similarly } \\
\text { oriented but their y-axes are oppositely } \\
\text { oriented relative to one another. Single } \\
\text { servos (throttle, nose landing gear) are ori- } \\
\text { ented the same as on the GeoSurv II pro- } \\
\text { totype. }\end{array}$ \\
\hline GAMSO & $\begin{array}{l}\text { Servo locations and orientations deter- } \\
\text { mined through the GAMSO strategy. }\end{array}$ \\
\hline
\end{tabular}




\subsubsection{Population Selection}

Evolutionary processes such as natural selection and mating are essential for producing populations that are adapted to their environment and are genetically diverse, ensuring that the best genes are carried over from one generation to the next. These processes are mimicked in genetic algorithms by the elite selection and crossover functions, respectively, whereby it is important that the variables which yield the best solutions are carried over to the next generation. Population selection is necessary to define the population of the succeeding generation. The mechanisms by which each generation, other than the initial generation, are defined are elite selection, crossover, and mutation. These mechanisms are used in varying fractions with respect to one another to create the population for the next generation.

\section{Elite Selection}

Elite selection refers to directly importing the best solutions of a previous generation directly into the current generation. This is always done by selecting the fittest individuals. The variation that one might see in this application is the fraction of the total population that is composed of elite individuals, also known as the elite fraction. This may be defined within the genetic algorithm to be a constant, or may vary depending on the genetic diversity of the population. It is important to maintain genetic diversity in order to avoid premature convergence to a local minimum instead of a global minimum. Additionally, the elite fraction may not be defined and instead the number of elite individuals are selected according to a threshold fitness, whereby individuals with fitness greater than the threshold are carried over directly to the next generation. Similar to the elite fraction, the threshold fitness may be predefined or can vary depending on the current population. 
In GAMSO, the elite fraction is defined in the options at the beginning of the GAMSOmaster.m script. Elite individuals, along with their fitness function values. are carried over directly into the next generation. The fitness function values of these individuals are never recalculated, which increases the speed of the GAMSO scheme.

\section{Crossover Selection}

Crossover selection relies on the choosing of two (or more) individuals with good fitness, which will then combine in some defined manner to form one (or more) children. The mechanisms used for crossover will be discussed in 8.2.5. The number of individuals selected for crossover is controlled by the crossover fraction which is the fraction of the remaining succeeding population (total population less elite individuals) that is to be filled by crossover children. The selection of individuals for crossover is most commonly created in one of two ways:

1. Roulette Wheel Selection: Individuals are assigned a probability of being selected for crossover based on their fitness. Individuals with the highest fitness have a higher probability of being selected. A uniform random number is then generated and the individual corresponding to that number is selected for crossover. Additional uniform random numbers are generated and individuals are selected until there are enough parents for crossover. The probability of an individual to be selected can be based either on its rank within the population or by its normalized fitness function value [35].

2. Tournament Selection: A subset of individuals is randomly chosen from the entire population and ranked by fitness function value. The fittest individual within the subset is selected to be a parent for crossover. This process is repeated until enough parents for crossover are generated. The size of the subset of individuals randomly selected may be constant or varying. 
According to Haupt and Werner [35], the probability of selection of individuals for rank order roulette wheel and tournament selection is nearly the same. Both methods are simple to implement. In GAMSO, tournament selection was used to select parents for crossover. The selection of parents for crossover is performed in the GAMSOselection.m script.

\section{Mutation Selection}

The selection of individuals for mutation is most often random, but it can be performed on a subset of the population such as the elite individuals. The number of individuals selected for mutation is the number of individuals needed to fill the remainder of the succeeding generation not defined by elite selection or crossover. In GAMSO, individuals are chosen randomly for mutation. This is done in the GAMSOselection.m script.

\section{Population Concatenation}

The population of the succeeding generation is compiled from the subpopulations of elite individuals, crossover children, and mutated individuals. In GAMSO the elite fraction and the crossover fraction are defined as constants in the main options structure of GAMSOmaster.m. A function was built in to GAMSO so that if the population remained stagnant, i.e. no improvement in the best fitness function over a defined number of generations, called stall generations, then the elite fraction and crossover fraction would be decreased. This was intended to allow for additional mutation, and hence additional genetic diversity within the population. This measure was implemented in order to avoid converging to local minima. 


\subsubsection{Population Crossover}

Crossover is the genetic algorithm equivalent of mating in evolution. It involves the combination of two (or more) individuals to create an offspring that differs from its parents. This essentially provides a means of combining favourable genes to produce a chromosome that is better than its parents. This is most often completed with two parents, but can be completed with $N$ parents, where $N$ is the number of genes within each chromosome.

There are many ways that the genes from parents may be combined to form a crossover child. Among others, the two most common methods are listed as follows:

1. Single-point crossover: A random integer, $i$, between 2 to $N-1$ is generated. All of the genes before gene $i$ are taken from parent \#1, and the remainder of the genes are taken from parent \#2. A second offspring may be generated by reversing the order of the parents.

2. Multi-point crossover: A random binary string of length $N$ is generated. The first offspring inherits genes from parent \#1 in all of the locations where the string has a value of 1 , and inherits genes from parent \#2 in the locations where the string has a value of zero. Again, a second offspring may be generated whereby a string value of 1 means inheritance from parent \#2, and a string value of zero means inheritance from parent \#1.

The multi-point crossover method was implemented in GAMSO. This was performed in the script GAMSOxover.m. Special care had to be taken with the orientation components of the chromosome, since the orientation is defined by the combined XYZ-rotation of the source. Simply mixing the orientation genes would result in offspring that are similar to neither parent, and hence potentially introduce undesirable solutions and even constraint violations. The entire orientation chromosome 
(XYZ-rotation) for each source was treated as a single gene during crossover.

\subsubsection{Population Mutation}

Mutation introduces variations into the chromosome of selected individuals. Random mutation within a population can help to inject diversity into the gene pool of a population. This is especially useful for populations which have converged onto local minima.

In GAMSO, this is completed by randomly selecting a certain number of individuals who will then have one or more of their genes altered. The chromosome of each individual may be broken up into the location chromosome and the orientation chromosome. The number of location and orientation genes that will be altered is chosen at random. Additionally, the location and orientation genes that are altered are also chosen at random. The genes are then altered by replacing them with random values which are generated using the same procedure as in population creation. Using the same gene creation method ensures that the mutated genes will not violate the bounds, whereas altering the genes through an operation such as multiplication with a random number can generate genes which cause boundary violations. Mutation in GAMSO is performed in the GAMSOmutation.m script.

\subsection{GAMSO Results}

This section presents the results of the GAMSO implementation. A study on the efficiency of GAMSO was conducted. The results of the theoretical optimized configuration were validated experimentally, as will be described in this section. 


\subsubsection{GAMSO Efficiency}

An investigation was conducted to evaluate GAMSO's ability to find the global minimum of a defined problem. For this investigation, additional constraints were placed on the GeoSurv II GAMSO definition, to produce a manageable number of potential solutions. All source locations, and the orientations of both \#2 elevator and \#2 rudder servos, the throttle servo, and the nose landing gear servo were fixed to their respective configurations on the GeoSurv II prototype. The orientation of both flaperon servos, as well as the \#1 elevator and \#1 rudder servos were able to assume any of 16 feasible orientations, yielding a problem with 65,536 unique solutions.

An exhaustive search was conducted in MATLAB to determine the global minimum of the problem. The exhaustive search took approximately 20 hours to complete, running on a computer with an Intel Core i5 2.67 GHz CPU. Each additional variable or degree of freedom multiplied the processing time proportional to the size of the allowable subset of values corresponding to the additional variable. The amount of time necessary to complete an exhaustive search of all possible solutions for the full GAMSO problem would lead to solution times on the order of years. For this reason, it is not feasible to determine the global minimum through an exhaustive search, thus an investigation was required to determine the ability of GAMSO to quickly reach a global minimum.

GAMSO was modified to meet the constraints required for this investigation. A total of 16 unique initial populations of 200 individuals were produced and run until each trial reached the global minimum found during the exhaustive search. All of the 16 trials reached the global minimum. The fewest number of generations required to reach the minimum was 7 , which occurred for nine trials. The largest number of generations needed was 278 , which occurred for a single trial. Each generation took 
roughly 4 minutes. The number of generations needed, as well as the approximate elapsed time to reach the global minimum for each trial is shown in Appendix C.

The constrained problem definition had a strong local minimum. which corresponded to the second best global solution, that caused GAMSO to stall for an extended number of generations, leading to decreased genetic diversity within the population. The genetic diversity was increased by altering the selection ratios as outlined in Section 8.2.4. For the constrained problem, GAMSO was able to quickly converge to the global minimum or to the strong local minimum within 28 generations for all trials. These results confirm that GAMSO is capable of efficiently locating the global minimum of the defined problem.

\subsubsection{GAMSO MATLAB Results}

The GAMSO package was used to determine the optimized configuration of the GeoSurv II prototype sources within the constraints discussed in Section 8.2.1. The optimal GAMSO solution was reached in 599 generations using a population of 200 chromosomes per generation, yielding a solution that is a significant improvement over the current GeoSurv II configuration.

For comparison, a chromosome representing the current configuration and a manually optimized configuration were analyzed using the same scripts as in the GAMSO package. The metric used to define the improvement of the optimized solution over the current solution is expressed as follows:

$$
\% \text { Improvement }=\frac{\left\|\vec{B}_{\text {current }}\right\|-\left\|\vec{B}_{\text {optımized }}\right\|}{\left\|\vec{B}_{\text {current }}\right\|} \times 100
$$


where $\vec{B}_{\text {current }}$ and $\vec{B}_{\text {optrmazed }}$ is the magnetic flux density at the centre of each magnetometer volume for the current and optimized configurations, respectively. Using (8.8), a positive value would indicate that the magnetic noise has decreased over the present value, and vice versa. The percent improvement of the theoretical results over the optimized results is provided in Table 8.2, which shows that the GAMSO strategy is capable of decreasing the wingtip magnetic noise to an even greater extent than just simple manual optimization.

Table 8.2: Comparison of theoretical results for the current configuration, manually optimized configuration, and the GAMSO optimized configuration. Results are presented for a zero background magnetic field. The magnetic noise, $\left\|\vec{B}_{\text {norse }}\right\|$, and percent improvement, \% Imp., are indicated.

\begin{tabular}{|c|c||c|c||c||c|c|}
\hline & \multicolumn{2}{|c|}{ Port } & \multicolumn{3}{c|}{ Starboard } \\
\hline Configuration & $\vec{B}_{\text {nonse }} \|[\mathrm{nT}]$ & \% Imp. & $\vec{B}_{\text {norse }}$ & {$[\mathrm{nT}]$} & \% Imp. \\
\hline \hline Current & 4.47 & - & 4.77 & - \\
\hline Manually Optimized & 1.06 & 76.3 & 0.56 & 88.2 \\
\hline GAMSO & 0.06 & 98.7 & 0.03 & 99.4 \\
\hline
\end{tabular}

A visualization of the GAMSO solution as compared to the current GeoSurv II prototype source configuration is shown in Figure 8.3. Although the positioning of sources in Figure 8.3 is approximate, it illustrates that although the positioning changes of each servo are not drastic, the optimized orientation of each source leads to the substantial improvement in wingtip magnetic noise. 


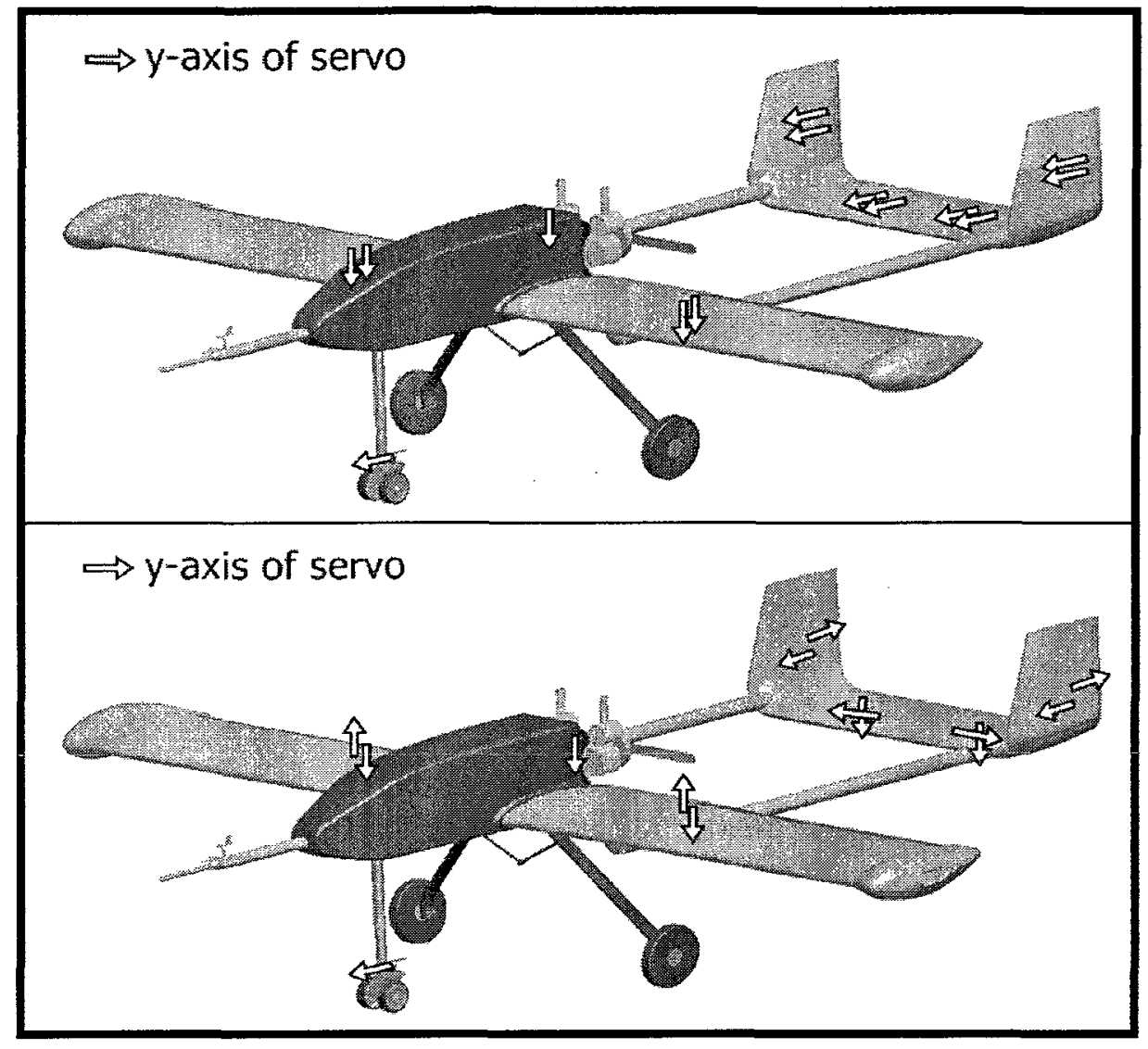

Figure 8.3: Illustration of servo locations and orientations for current (top) and GAMSO optimized (bottom) configurations. The position of each servo is located approximately at the base of the corresponding arrow.

In addition to improvement in the attenuation of wingtip magnetic fields, the GAMSO solution also exhibited greater spatial uniformity over the other two configurations. This may be seen by comparing Figures 8.4 to 8.6 which show the total magnetic field contours in $\mathrm{nT}$ for the coordinate planes intersecting the centre of each magnetometer domain. 

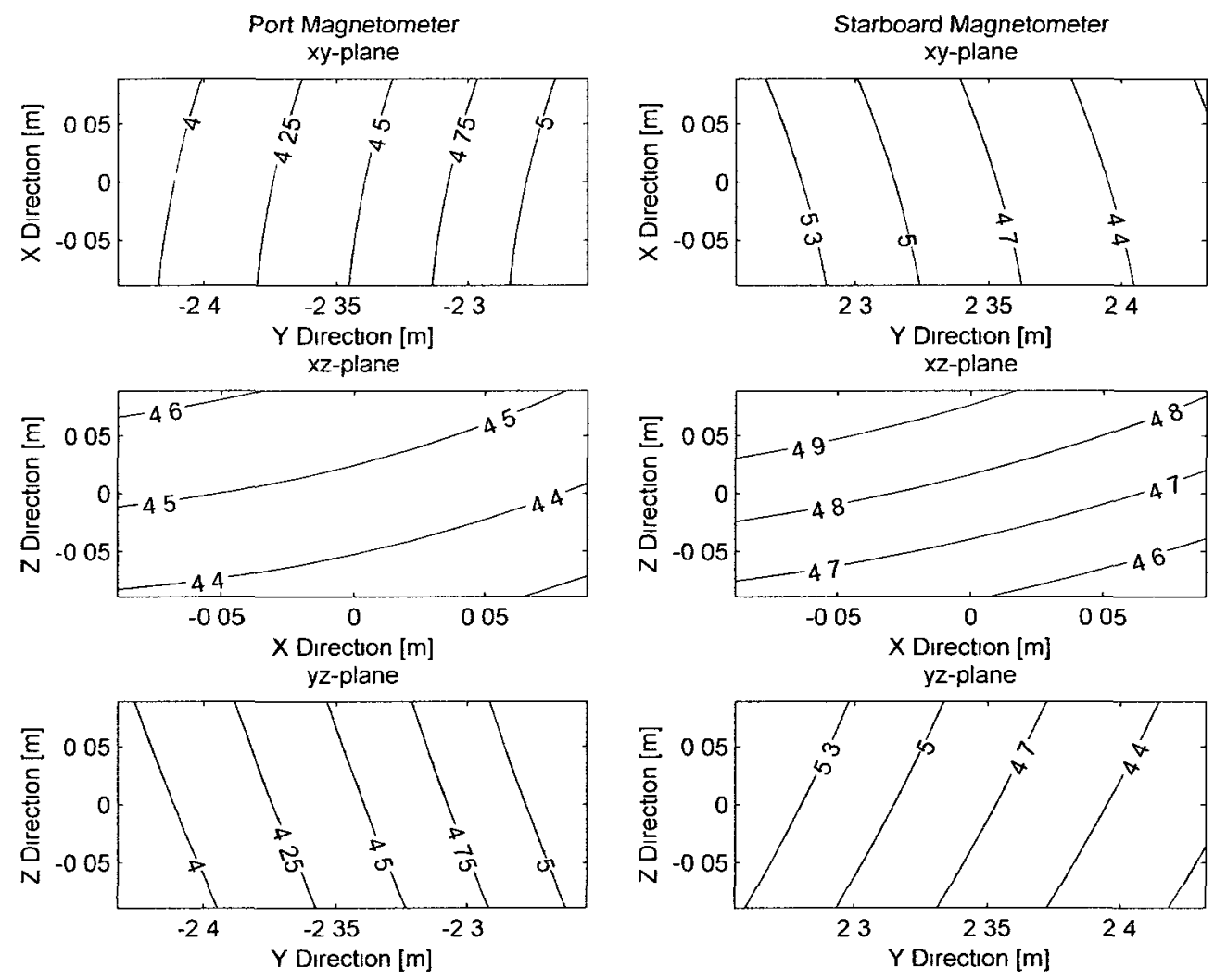

Figure 8.4: Magnetic field at wingtip magnetometers for the current servo configuration along coordinate planes intersecting the centre of each magnetometer domain. All values in $\mathrm{nT}$. 

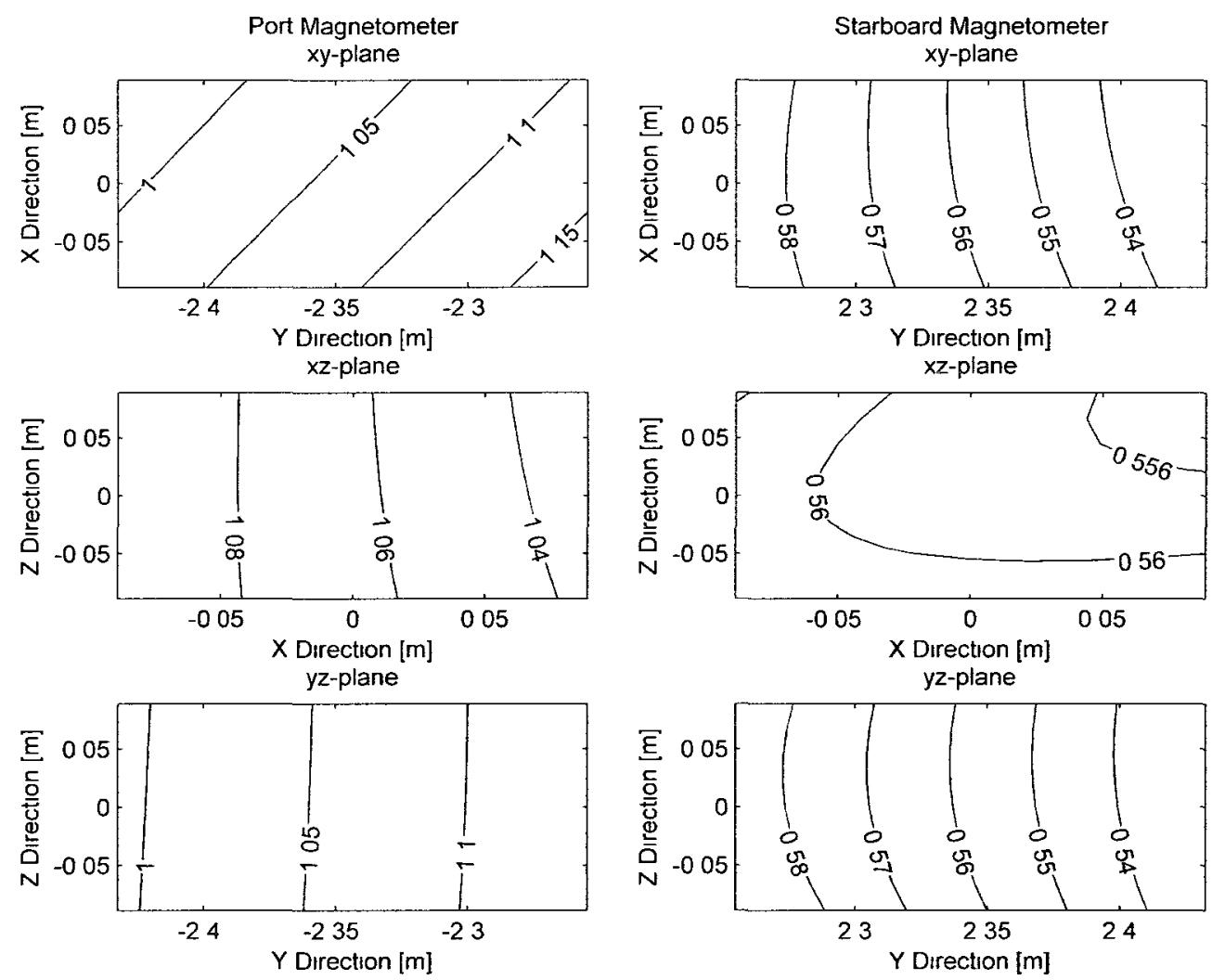

Figure 8.5: Magnetic field at wingtip magnetometers for the manually optimized servo configuration along coordinate planes intersecting the centre of each magnetometer domain. All values in $\mathrm{nT}$. 

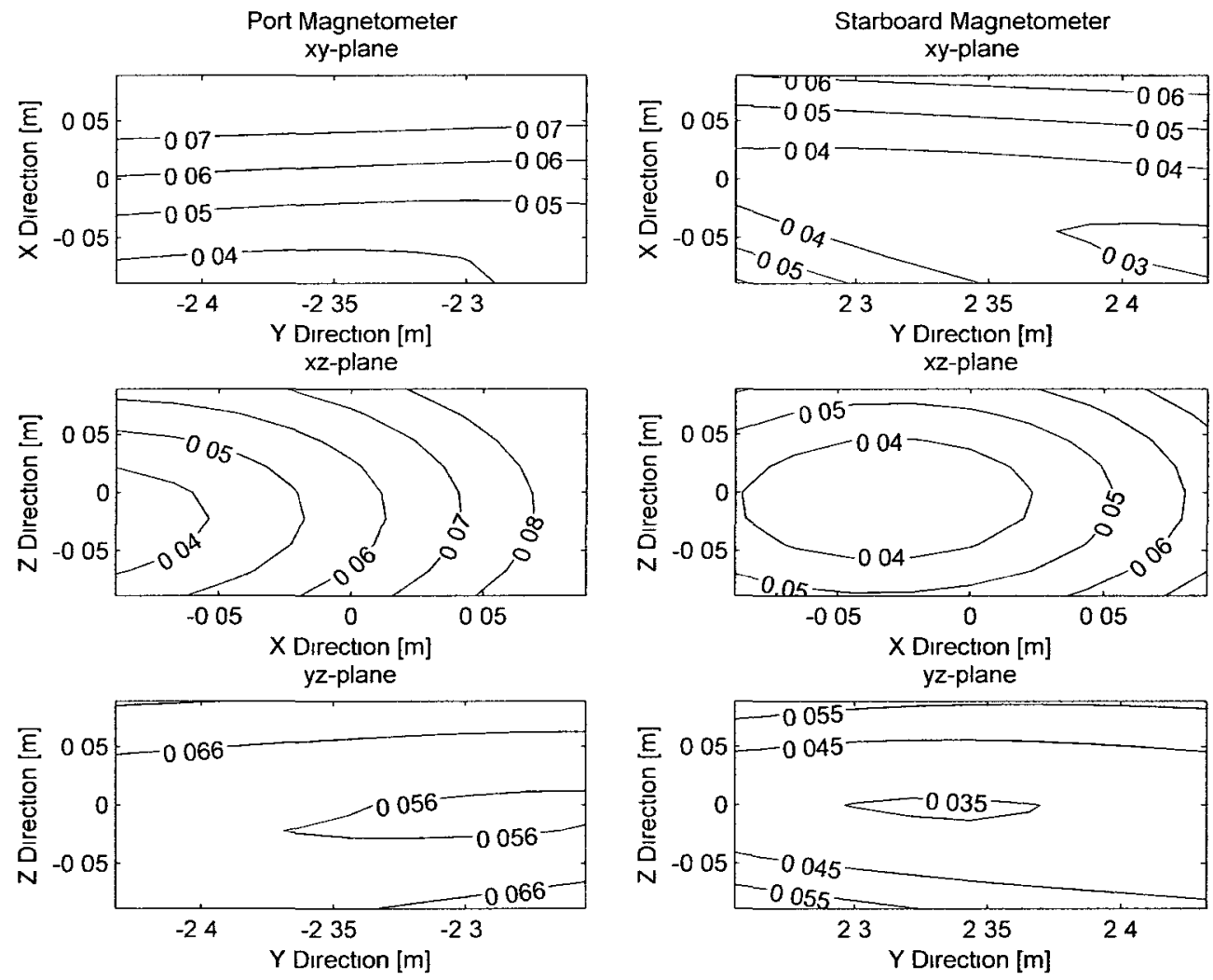

Figure 8.6: Magnetic field at wingtip magnetometers for the GAMSO optimized servo configuration along coordinate planes intersecting the centre of each magnetometer domain. All values in nT. 
Furthermore, it should be noted that the result of the optimized GAMSO solution does not decrease the magnetic field at every point around the UAS, but simply configures sources to minimize the magnetic field at the magnetometer locations. This result is illustrated in Figures 8.7 and 8.8 which show the total magnetic flux density contours for the current and GAMSO optimized configurations in xy-plane of the UAS. Note that the magnetic flux density is still large near sources since it is impossible to completely cancel any source.

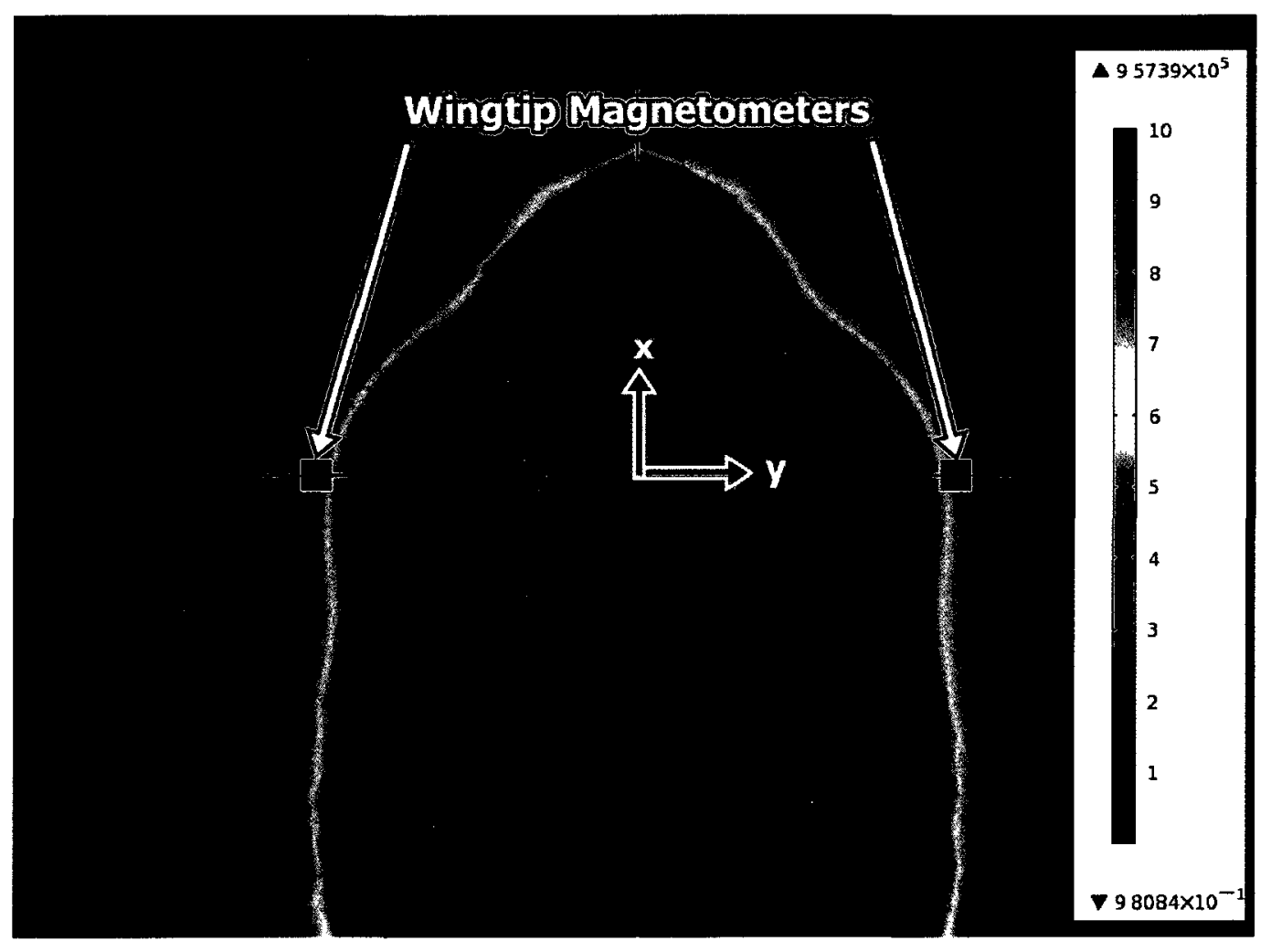

Figure 8.7: Total magnetic field contour in the xy-plane for current configuration generated using COMSOL Multiphysics. There are large magnetic fields and magnetic field gradients at wingtip magnetometer locations. All values in $\mathrm{nT}$. 


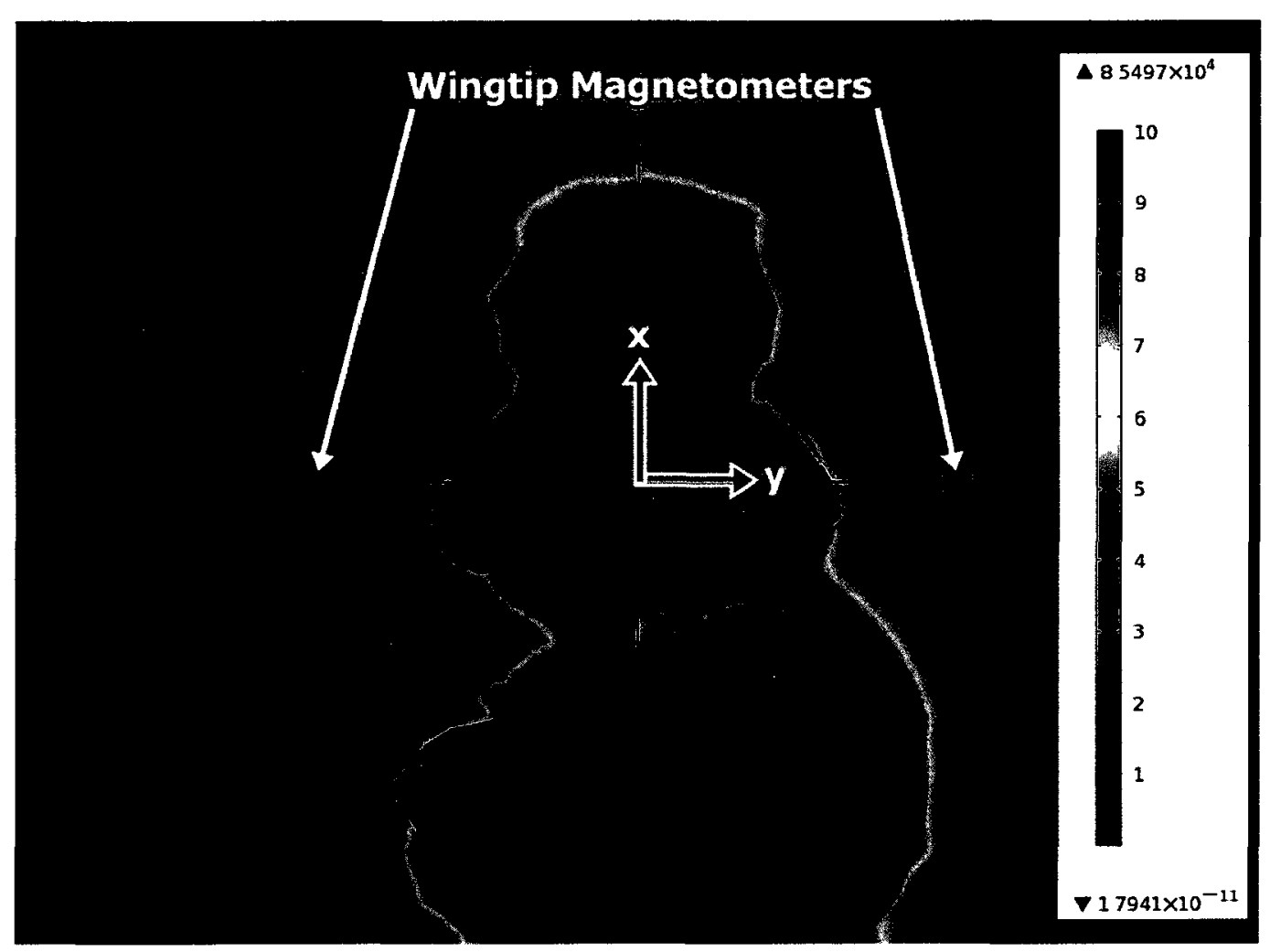

Figure 8.8: Total magnetic field contour in the xy-plane for GAMSO optimized configuration generated using COMSOL Multiphysics. Magnetic field is shaped to minimize the gradient and total field at wingtip magnetometer locations. All values in $\mathrm{nT}$.

\subsubsection{GAMSO Experimental Validation}

An experimental validation was conducted at the Geomagnetic Laboratory by configuring 13 Hitec HSR-5990TG servos in the current, manually optimized, and GAMSO optimized configurations with respect to the wingtip magnetometers. Supports were created to allow for the approximate positioning of each servo and magnetometer. Due to only 13 servos being available at the time of testing, the nose landing gear servo was not represented.

The experimental setup required the creation of a grid representing the coordinate system of the UAS, and mapping of the locations of each servo and magnetometer 
support. Servo and magnetometer locatıons were estimated to be within $\pm 0.05 \mathrm{~m}$, and servo orientations were estimated to be within $\pm 20^{\circ}$ of their respective configurations Three Geometrics G-822A Cesium magnetometers were utilized: one at each wingtip location, and the third magnetometer was located outside the test building and used as a reference sensor A fluxgate magnetometer was located $1 \mathrm{~m}$ aft of each wingtıp magnetometer to monitor the magnetic field vector components The configuration of the current and GAMSO configurations is shown in Figures 8.9 and 810 taken during testing.

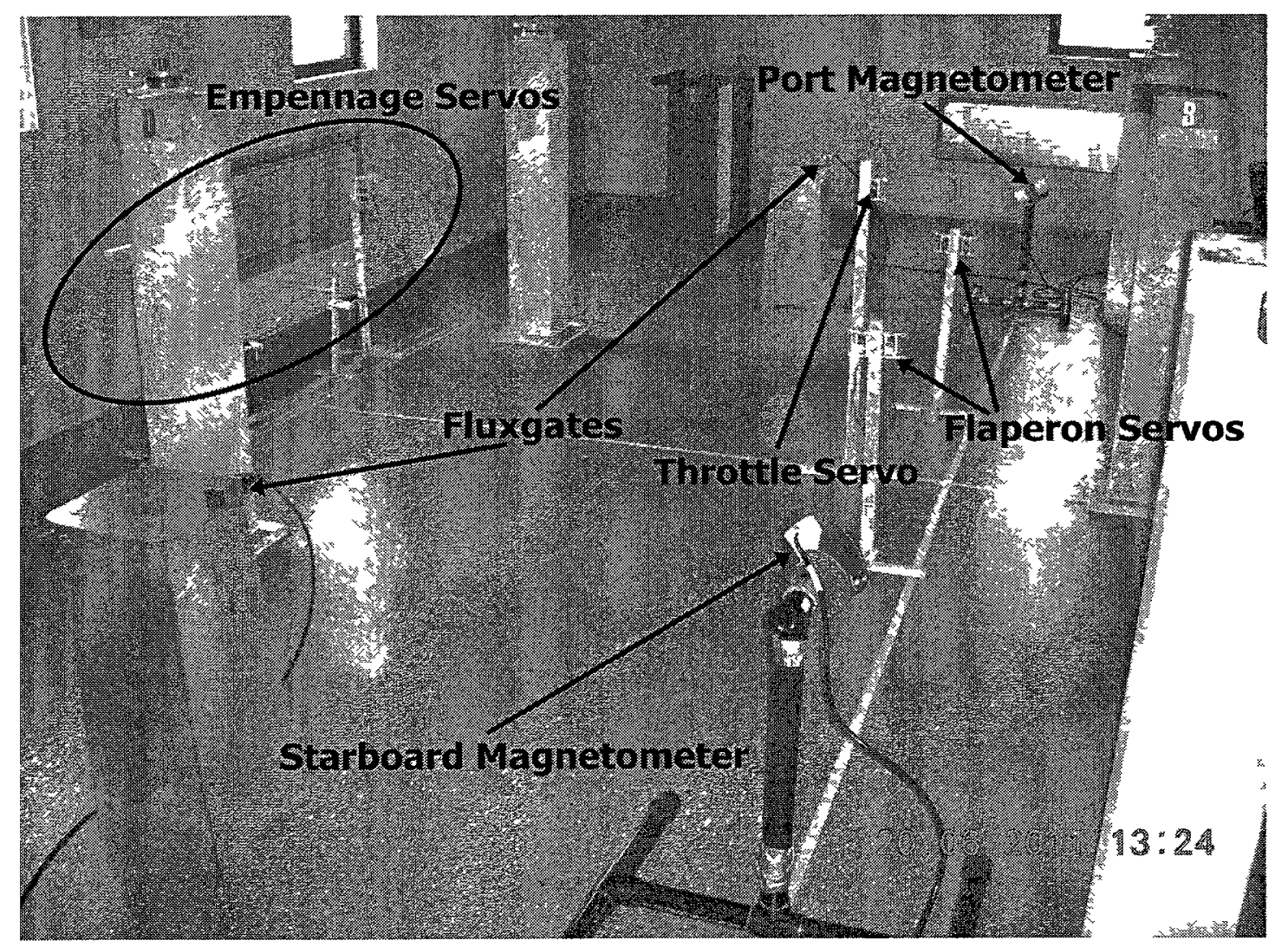

Figure 8.9: Current configuration used durng GAMSO validation as shown from the starboard wingtip location. 


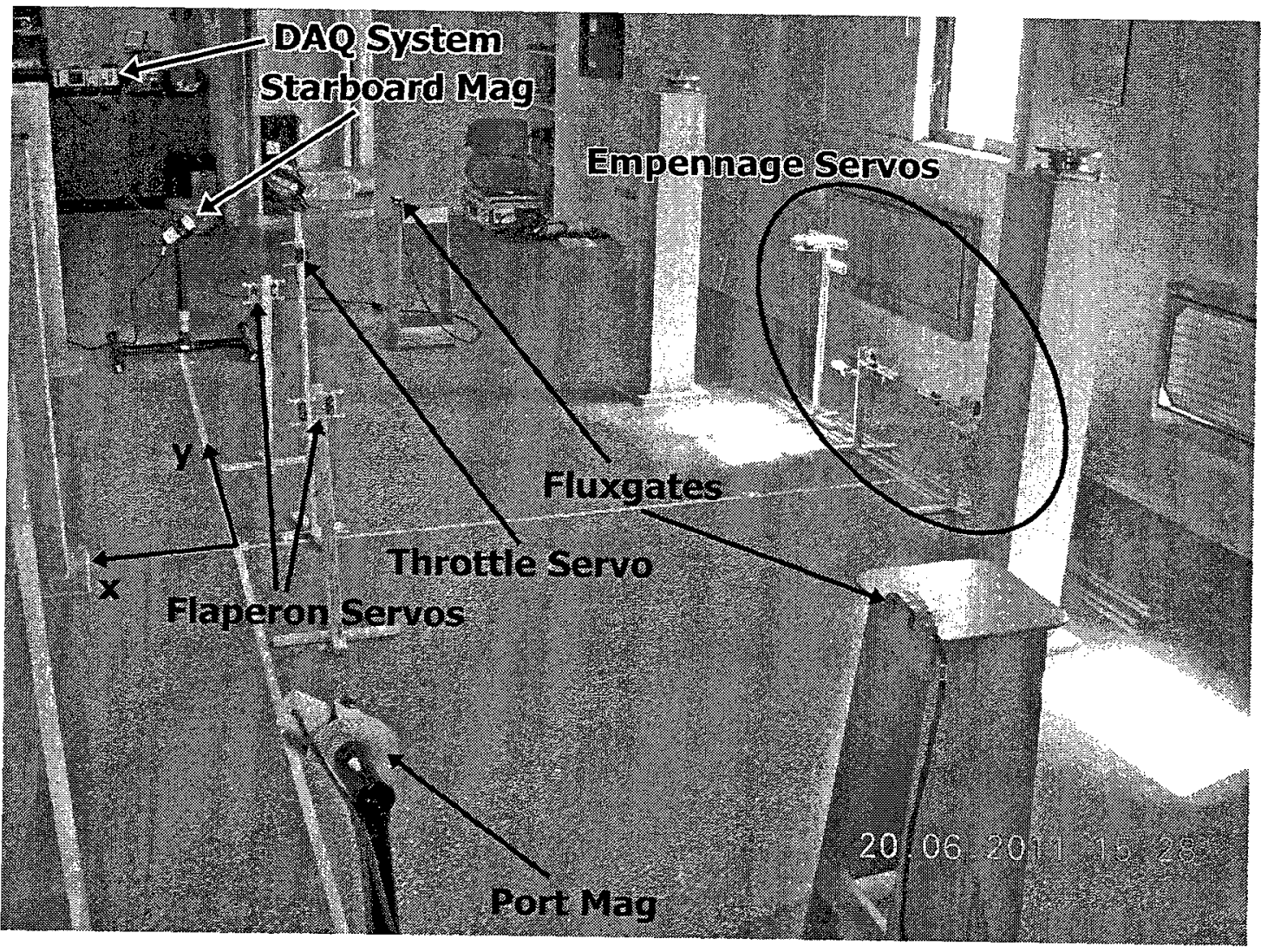

Figure 8.10: GAMSO optimized configuration used during GAMSO validation as shown from the port wingtip location. 
The experimental procedure involved obtaining a baseline reading before each measurement, during which all servos were moved to a distance of at least from $4 \mathrm{~m}$ from the nearest magnetometer. The servo supports were then quickly brought into the test area, and configured on the predefined grid to simulate the respective configurations. A measurement was taken of the magnetic field at all magnetometers, and the magnetic noise was calculated as the difference between the baseline reading and the measurement with servos present, and corrected for any changes in the background field. For each configuration. this process was repeated multiple times to average out any positioning or orientation errors, and the mean of all noise measurements was treated as the true noise value.

The results of the experimental investigation show that the GAMSO solution is indeed capable of significantly reducing the magnetic field at the wingtip magnetometer locations. Table 8.3 compares the theoretical and experimental results for the current, manually optimized, and GAMSO optimized configurations for the 13 servo scenario. The values in Table 8.3 differ from Table 8.2 due to the absence of the nose landing gear servo. Additionally, the theoretical magnetic noise values have been corrected for the magnetic vector field used during the respective measurements. 
Table 8.3: Comparison of theoretical and experimental results for the GAMSO algorithm. The current GeoSurv II prototype configuration, as well as the manually optimized and GAMSO solution are compared. The theoretical (Theo.) and experimental (Exp.) percent improvement are measured relative to the the current theoretical and experimental wingtip noise values, respectively. Results are presented for the background field measured during testing. The magnetic noise, $\left\|\vec{B}_{\text {noise }}\right\|$, and percent improvement, \% Imp., are indicated.

\begin{tabular}{|c|c|c|c|c|c|c|c|}
\hline & & \multicolumn{3}{|c|}{ Port } & \multicolumn{3}{|c|}{ Starboard } \\
\hline Config. & Case & $\vec{B}_{\text {noise }}$ & {$[\mathrm{nT}]$} & \% Imp. & $\vec{B}_{\text {nonse }}$ & {$[\mathrm{nT}]$} & \% Imp. \\
\hline \multirow{2}{*}{ Current } & Theo. & \multicolumn{2}{|c|}{3.36} & - & \multicolumn{2}{|c|}{4.87} & - \\
\hline & Exp. & \multicolumn{2}{|c|}{3.30} & - & \multicolumn{2}{|c|}{5.09} & - \\
\hline \multirow{2}{*}{$\begin{array}{l}\text { Manually } \\
\text { Optimized }\end{array}$} & Theo. & \multicolumn{2}{|c|}{0.43} & 87.2 & \multicolumn{2}{|c|}{0.29} & 94.0 \\
\hline & Exp. & \multicolumn{2}{|c|}{0.40} & 87.9 & \multicolumn{2}{|c|}{0.33} & 93.5 \\
\hline \multirow{2}{*}{ GAMSO } & Theo. & \multicolumn{2}{|c|}{0.23} & 93.2 & \multicolumn{2}{|c|}{0.03} & 99.4 \\
\hline & Exp. & \multicolumn{2}{|c|}{0.47} & 85.8 & 0.0 & & 98.8 \\
\hline
\end{tabular}

\subsection{GAMSO Conclusions}

Reasons for the discrepancy between theoretical and experimental results stems from two main sources: experimental and modelling errors. The experimental errors are due to improper positioning and orientation of servos and magnetometers during experimentation. Also, included in experimental errors are different magnetic field gradients at each wingtip magnetometer, a scenario which is difficult to avoid. The modelling errors are due to the utilization of a servo model that is not an exact representation of each servo, and using only a single representative servo to model all 14 servos. The results from the investigation outlined in Section 4.3.2 show that there is a roughly $11 \%$ variability from the mean in the magnitude of the magnetic signatures of the 13 Hitec HSR-5990TG servos used during experimental validation, meaning that in order to more accurately model the magnetic field of the UAS, a 
separate model for each individual servo must be generated.

The GAMSO strategy was proven to significantly reduce the wingtip magnetic noise while maintaining a servo configuration that is feasible from an aircraft performance standpoint. Although the magnetic noise reduction measured experimentally was not as great as that found theoretically, the results still indicate that GAMSO is an effective strategy. The GAMSO strategy may be expanded to include additional sources and configurations, making it a useful tool for many applications.

Recommendations to further improve this strategy involve individually characterizing each magnetic field source (each servo) for inclusion into the GAMSO script. Servo pairings should consist of servos with similar magnetic signatures. Additionally, improvement of the constraint and stall generation handling methods to more sophisticated schemes may improve the efficiency and performance of the strategy. 


\section{Chapter 9}

\section{Conclusions and Recommendations}

\subsection{Summary}

Magnetic signature reduction of a geomagnetic surveying unmanned aircraft system (UAS), GeoSurv II, is presented in this thesis. The magnetic noise generated by onboard sources at magnetometer locations must be attenuated to avoid contamination of survey data. This thesis outlines the results of an investigation into the magnetic signature of individual GeoSurv II components, as well as full scale testing of GeoSurv II to quantify the magnetic signature of the current prototype. This investigation was followed by the development of two characterization schemes, permanent magnetic dipole (PMaD) and dual permanent magnetic dipole (DuPMaD), which were used to model simple magnetic field sources using magnetic point dipoles.

Both characterization schemes were able to adequately model two different servo models by matching experimental data to dipole models. The experimental data was collected using a custom built test apparatus, the MMTA-3D, which allowed for measurement points at thousands of precisely known locations around smaller 
components. The results of PMaD and DuPMaD schemes were validated through a simulation in the COMSOL Multiphysics software package. Both the PMaD and DuPMaD schemes provided an accurate representation of each servo and yielded nearly identical results, leading to the recommendation of the PMaD scheme for modelling simple magnetic noise sources.

The servo models developed using the characterization schemes were used for both a cancellation strategy and source configuration optimization strategy. The cancellation strategy known as the cancellation magnet configuration (CMC) strategy utilized a permanent magnet close to each source to yield a reduced net magnetic effect. The position and orientation of the cancellation magnet relative to each source was optimized to minimize the net magnetic far-field. The CMC strategy was experimentally validated which yielded reductions in the total magnetic field of $56.1 \%, 68.1 \%$, and $76.2 \%$ at source-sensor distances of $0.22 \mathrm{~m}, 0.33 \mathrm{~m}$, and $0.52 \mathrm{~m}$, respectively.

Lastly, the genetic algorithm source configuration optimization (GAMSO) strategy was developed to optimize the configuration of magnetic noise sources to minimize magnetic noise at wingtip magnetometer locations using a genetic algorithm. A full scale model simulating the configuration of all servos onboard GeoSurv II was utilized, and respective bounds were established. Furthermore, the orientations of each servo were constrained such that all servos were normal to the coordinate system of the UAS, ensuring that the mechanical leverage of each servo could be readily utilized. The GAMSO strategy was experimentally validated, yielding a reduction of magnetic noise of $98.8 \%$ and $85.8 \%$ for the starboard and port magnetometer locations, respectively, relative to the current configuration. 


\subsection{Conclusions}

This section outlines the major conclusions and contributions from this research project.

1. Current State of GeoSurv II Magnetic Signature: Full scale testing conducted at the Geomagnetic Laboratory revealed that the magnetic noise at the wingtips of the current GeoSurv II prototype is at least one order of magnitude above design specifications; therefore, in its current state, the GeoSurv II UAS is unfit for geomagnetic survey missions. The UAS components responsible for the largest magnetic noise contribution are the servo actuators and engine assembly. The reduction of the wingtip magnetic noise to within design specifications will require the use of multiple mitigation strategies. Specific recommendations to meet design specifications are outlined in Section 9.3.

\section{Genetic Algorithm Source Configuration Optimization (GAMSO):} The GAMSO strategy developed in this thesis resulted in a significant reduction of wingtip magnetic noise from the onboard servo actuators. The results were experimentally validated which yielded magnetic noise reductions of $98.8 \%$ and $85.8 \%$ for the starboard and port wingtip magnetometer locations, respectively, relative to the current GeoSuv II prototype configuration. This approach was designed to produce practical servo configurations which could be readily implemented on GeoSurv II.

3. Passive Cancellation Strategy: The novel Cancellation Magnet Configuration $(\mathrm{CMC})$ strategy was proven to significantly reduce the magnetic signature of an individual component through the use of a cancellation magnet. The CMC strategy involved determining the optimal position and orientation of a cancellation magnet relative to each source to yield a minimized net magnetic 
far-field effect. The CMC strategy was implemented for a servo actuator and experimentally validated, resulting in a $76.2 \%$ reduction in the total magnetic field at a source-sensor distance of $0.52 \mathrm{~m}$. This approach is most beneficial for unpaired sources, since paired sources may be counteroriented relative to one another to reduce the net magnetic effect.

4. Modelling Simple Magnetic Sources: The PMaD and DuPMaD modelling strategies were developed as an extension of modelling strategies previously developed by Wells [1]. It was found that the PMaD and DuPMaD schemes were better able to model the vector components of simple magnetic field sources by fitting dipole models to data collected experimentally using a novel test apparatus. The PMaD and DuPMaD approaches yielded nearly identical results. Since the PMaD scheme is simpler, it was concluded that it should be the characterization strategy of choice for input into models and optimization schemes.

\subsection{Recommendations}

An experimental investigation of the GeoSurv II UAS led to the conclusion that the magnetic signature of the current prototype is above design specifications by at least one order of magnitude. It is necessary for the GeoSurv II to have a magnetic signature within design specifications to perform geomagnetic survey missions capable of producing useful data. The implementation of magnetic signature control strategies is of vital importance to the success of GeoSurv II as a geomagnetic survey aircraft.

The recommendations generated from this thesis project for the GeoSurv II design are as follows: 
1. Replace all ferromagnetic components with nonmagnetic counterparts. Although the selection of materials for GeoSurv II requires that the use of metals be kept to a minimum, there are still a significant number of GeoSurv II fasteners that are ferromagnetic. An investigation identifying these components was discussed in [30], which includes a list of over 100 components that should be replaced. Proper attention should be given to the initial selection of components to avoid the use of magnetic materials. A control system tracking current and allowable materials onboard GeoSurv II would be beneficial for this purpose. Also, the configuration of the UAS (components and component configurations) should be retested periodically to ensure that components do not pose a problem.

2. All GeoSurv II wiring runs should be in twisted pair configuration. Twisting or braiding the wiring runs reduces the magnetic field by essentially creating a series of magnetic dipoles of alternating polarity.

3. Implementation of GAMSO strategy. The utilization of the GAMSO strategy is proven to significantly reduce the magnetic signature in regions of interest. An optimized configuration of the current Hitec HSR-5990TG servos was generated for the current prototype configuration. The GAMSO strategy should be used for any future design iterations involving the addition of magnetic components or revised control surface definitions. An attempt should be made to move all magnetic field sources as far away from each magnetometer as possible, which may include relocating the flaperon servos to the fuselage.

4. Implementation of CMC strategy. The CMC strategy will be most useful for unpaired magnetic noise sources such as the throttle servo and nose landing gear servo. A paired servo will behave as a cancellation magnet for its partner servo, and vice versa. if properly configured. This strategy should be performed 
in conjunction with GAMSO to reduce the magnetic signature of the UAS to the greatest extent possible.

By ensuring that magnetic signature control is an integral part of all GeoSurv II design decisions, it is the opinion of this author that achieving allowable wingtip magnetic noise values as specified in the systems requirements document [3] for GcoSurv II is entirely feasible.

\subsubsection{Future Work}

Recommendations for future work include:

1. Further investigation of low magnetic signature actuation strategies. Due to the current state of actuation options, a low magnetic signature actuator suitable for GeoSurv II will likely require in-house development. An investigation into piezoelectric actuators was conducted as part of this project. It was concluded that although piezoelectric actuators have a low magnetic signature (found to be negligible during experimentation), the present state of the technology does not allow for simple implementation into the GeoSurv II design; the displacements and forces generated by piezoelectric actuators are not fit for GeoSurv II's requirements.

2. Development of an active cancellation strategy to cancel transient magnetic fields. This strategy will likely involve pairing cancellation induction coils to sources, and generating opposing transient magnetic fields. Knowledge of the transient magnetic field from each source will be necessary to produce an appropriate cancellation field. This will require either a model based control system, or a feedback control system, each with their own inherent difficulties. A model based system would require a priori knowledge of the behaviour of the source, 
which may be difficult since it was observed in this thesis that the transient magnetic field response was not constant cycle-to-cycle for active servo actuators. Alternatively, a feedback control system would require the measurement of very small magnetic fields in close proximity to sources, while filtering the varying background field that would be present during missions due to flying over a survey area. 


\section{List of References}

[1] Matthew Wells. Attenuating magnetic interference in a UAV system. Master's thesis, Carleton University, 2008.

[2] R. Caron, C. Samson, P. Straznicky, S. Ferguson, R. Archer, and L. Sander. Magnetic and magneto-gradiometric surveying using a simulated unmanned aircraft system. 81st Annual Meeting of the Society of Exploration Geophysicists, 2011. Future publishing date.

[3] Project. GeoSurv II system requirements document: Revision E, March 2008. Carleton University Department of Mechanical and Aerospace Engineering.

[4] J.M.D. Coey. Magnetism and Magnetic Materials. Cambridge University Press, 2010.

[5] Sabri Cetinkunt. Mechatronics. Jọn Wiley \& Sons, 2007.

[6] John J. Holmes. Reduction of a Ship's Magnetic Field Signatures. Morgan \& Claypool Publishers, 2008.

[7] David Jiles. Introduction to Magnetism and Magnetic Materials. Chapman and Hall, 1991.

[8] Raluca Morjan and Sergey Prasalovich. EM4: Magnetic Hysteresis. Technical report, Chalmers University of Technology and Goteborg University, 2003. Lab Manual.

[9] T.J. Sumner, J.M. Pendlebury, and K.F. Smith. Conventional magnetic shielding. Journal of Physics D: Applied Physics, 20(9):1095-1101, 1987.

[10] Robert G. Olsen and Christopher E. Lyon. Modelling of extremely low frequency magnetic field sources using multipole techniques. IEEE Transactions on Power Delivery, 11(3):1563-1571, 1996. 
[11] R.F. Wiegert and J.W. Purpura. Magnetic scalar triangulation and ranging system for autonomous underwater vehicle based detection, localization and classification of magnetic mines. Oceans '04 MTTS/IEEE Techno-Ocean '04, 2:890896, 2004.

[12] Luciano E. Zaffanella, Thomas P. Sullivan, and Ivano Visitainer. Magnetic field characterization of electrical appliances as point sources through in situ measurements. IEEE Transactions on Power Delivery, 12(1):443-450, 1997.

[13] Wallace H. Campbell. Introduction to Geomagnetic Fields. Cambridge University Press, 1997.

[14] George Backus, Robert Parker, and Catherine Constable. Foundations of Geomagnetism. Cambridge University Press, 1996.

[15] Jerzy Jankowski and Christian Sucksdorff. Guide for magnetic measurements and observatory practice. Technical report, International Association of Geomagnetism and Aeronomy, 1996. Warsaw.

[16] Kok-Meng Lee and Hungsun Son. Distributed multipole model for design of permanent-magnet-based actuators. IEEE Transactions on Magnetics, 43(10):3904-3913, 2007.

[17] Thomas N. Roy. Spacecraft magnetic field modeling. IEEE Transactions on Magnetics, 13(1):914-920, 1977.

[18] Klaus Mehlem. Multiple magnetic dipole modeling and field prediction of satellites. IEEE Transactions on Magnetics, 14(5):1064-1071, 1978.

[19] A.V. Kilidshev, J.A. Nyenhuis, D.L. Boyko, P.N. Dobrodeyev, and S.A. Volokhov. Application of magnetic signature processing to magnetic center pinpointing in marine vehicles. OCEANS '99 MTS/IEEE, 3(1):1532-1536, 1999.

[20] Roelof Versteeg, Mark McKay, Matt Anderson, Ross Johnson, Bob Selfridge, and Jay Bennett. Feasibility study for an autonomous UAV-magnetometer system. Technical Report Final Report on SERDP SEED 1509:2206, Idaho National Laboratory, 2007.

[21] B. Ginzburg, L. Frumkis, B.Z. Kaplan, A. Sheinker, and N. Salomonski. Investigation of advanced data processing technique in magnetic anomaly detection systems. International Journal of Smart Sensing and Intelligent Systems, $1(1): 110-122,2008$. 
[22] A. Sheinker, B. Lerner, N. Salomonski, B. Ginzburg, L. Frumkis, and B.Z. Kaplan. Localization and magnetic moment estimation of a ferromagnetic target by simulated annealing. Measurement Science and Technology, 18(11):3451-3457, 2007.

[23] R. Alimi, N. Geron, E. Weiss, and T Ram-Cohen. Ferromagnetic mass localization in check point configuration using a Levenberg Marquardt Algorithm. Sensors, 9(11):8852-8862, 2009.

[24] J.T. Bono, D.J. Overway, and W.M. Wynn. Magnetic sensor operation onboard a UUV: magnetic noise investigation useing a total-field gradiometer. OCEANS '03 Proceedings, 4:2018-2022, 2003.

[25] G.I. Allen, J. Purpura, and D. Overway. Measurement of magnetic noise characteristics on select AUVs with some potential mitigation techniques. OCEANS '02 MTS/IEEE, 2(1):978 -984, 2002.

[26] G.I. Allen, R. Matthews, and M. Wynn. Mitigation of platform generated magnetic noise impressed on a magnetic sensor mounted in an autonomous underwater vehicle. OCEANS '01 MTS/IEEE, 1(1):63-71, 2001.

[27] Robert Forrester. Investigation into the effect of magnetic shielding of magnetic field sources on the background magnetic field. Technical report. Carleton University, 2011.

[28] Pavel Ripka. Magnetic Sensors and Magnetometers. Artech House, 2000.

[29] Robert Forrester. GeoSurv II magnetic signature control test procedures, results, and analysis. Technical report, Carleton University, 2011.

[30] Robert Forrester. Design change recommendations from magnetic signature control project. Technical report, Carleton University, 2010. DR No. 137-02.

[31] Kenichi Yamazaki and Tadashi Kawamoto. Simple estimation of equivalent magnetic dipole moment to characterize ELF magnetic fields generated by electric appliences incorporating harmonics. IEEE Transactions on Electromagnetic Compatibility, 43(2):240-245, 2001.

[32] John J. Holmes. Modeling a Ship's Ferromagnetic Signatures. Morgan \& Claypool Publishers, 2007.

[33] John H. Holland. Adaptation in Natural and Artificial Systems. University of Michigan Press, 1975. 
[34] David E. Goldberg. Genetic Algorithms in Search, Optimization, and Machine Learning. Addison-Wesley, 1988.

[35] Randy L. Haupt and Douglas H. Werner. Genetic Algorithms in Electromagnetics. John Wiley and Sons, Inc., 2007.

[36] Sancho Salcedo-Sanz. A survey of repair methods used as constraint handling techniques in evolutionary algorithms. Computer Science Review, 3(3):175 - 192, 2009. 
Appendix A

Component Testing Figures 

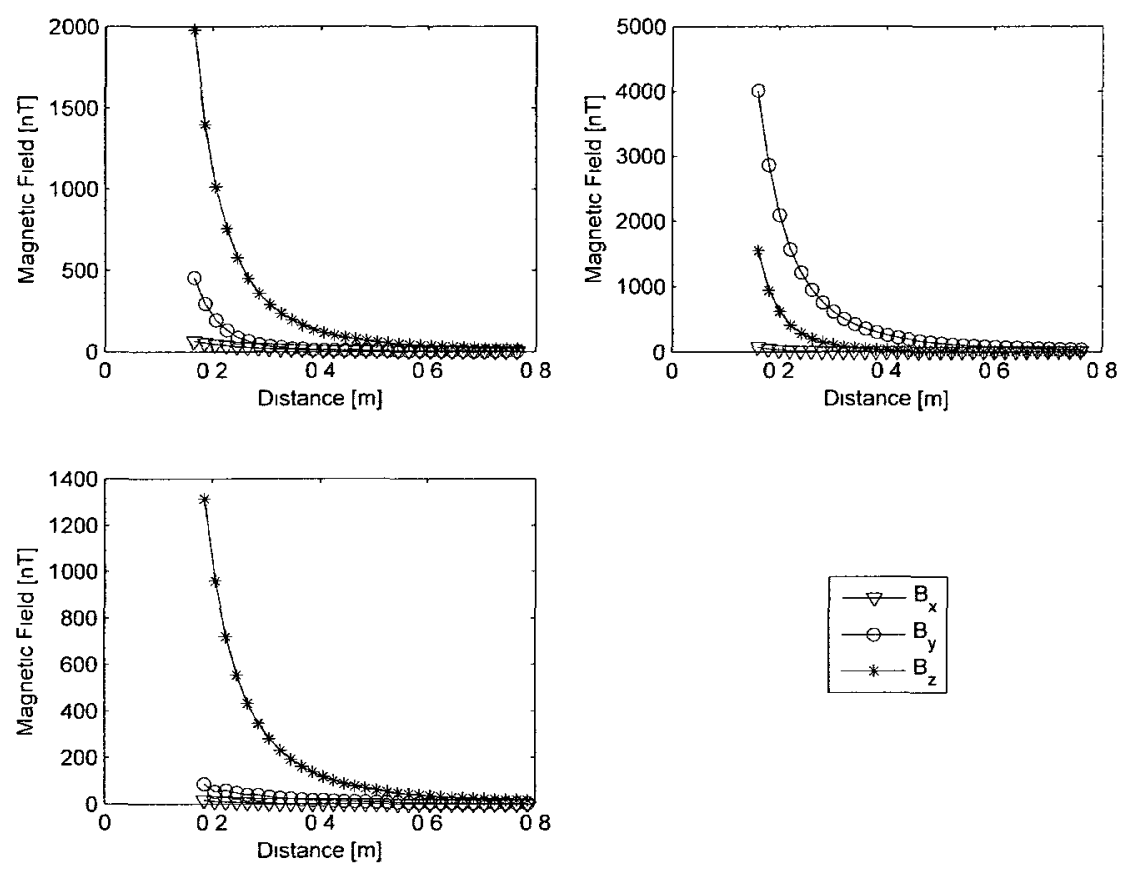

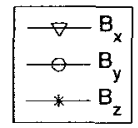

Figure A.1: Magnetic field rate of decay results for a single Hitec HSR-5990TG servo. Increasing distance along the $\mathrm{x}$ - (top left), $\mathrm{y}$ - (top right), and z- (bottom left) axis of a single servo. 

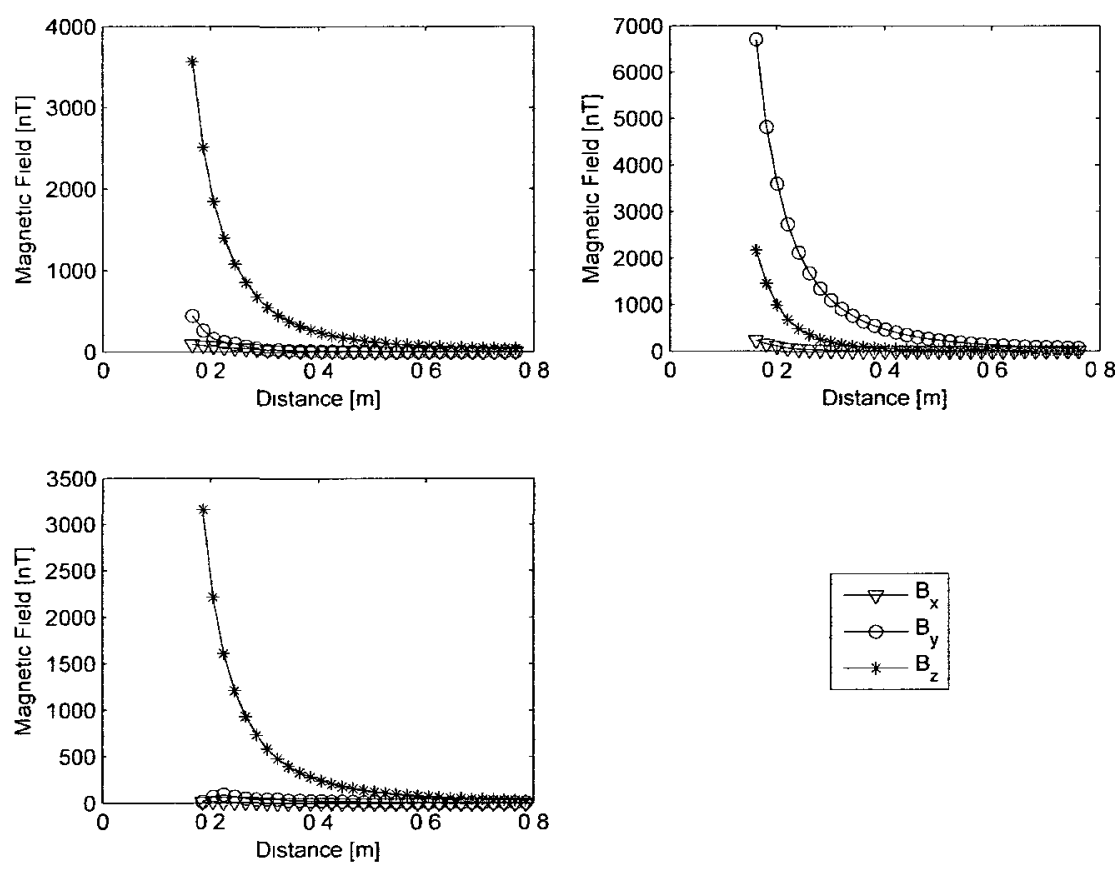

$$
\begin{array}{rr}
\nabla & B_{x} \\
-0 & B_{y} \\
* & B_{z}
\end{array}
$$

Figure A.2: Magnetic field rate of decay results for dual Hitec HSR-5990TG servos. Increasing distance along the $\mathrm{x}$ - (top left), $\mathrm{y}$ - (top right), and $\mathrm{z}$ - (bottom left) axis of a single servo. 

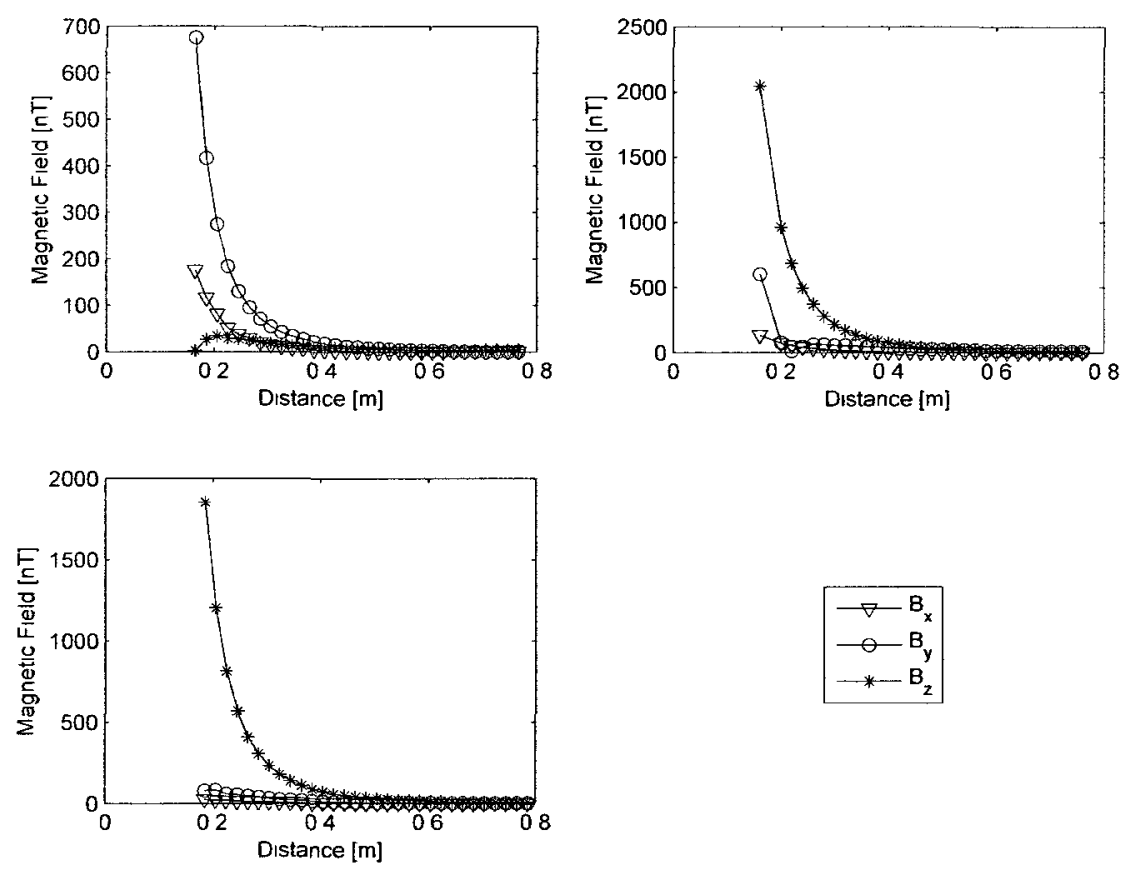

Figure A.3: Magnetic field rate of decay results for dual counteroriented Hitec HSR5990TG servos. Increasing distance along the $\mathrm{x}$ - (top left), $\mathrm{y}$ - (top right), and z- (bottom left) axis of a single servo. 


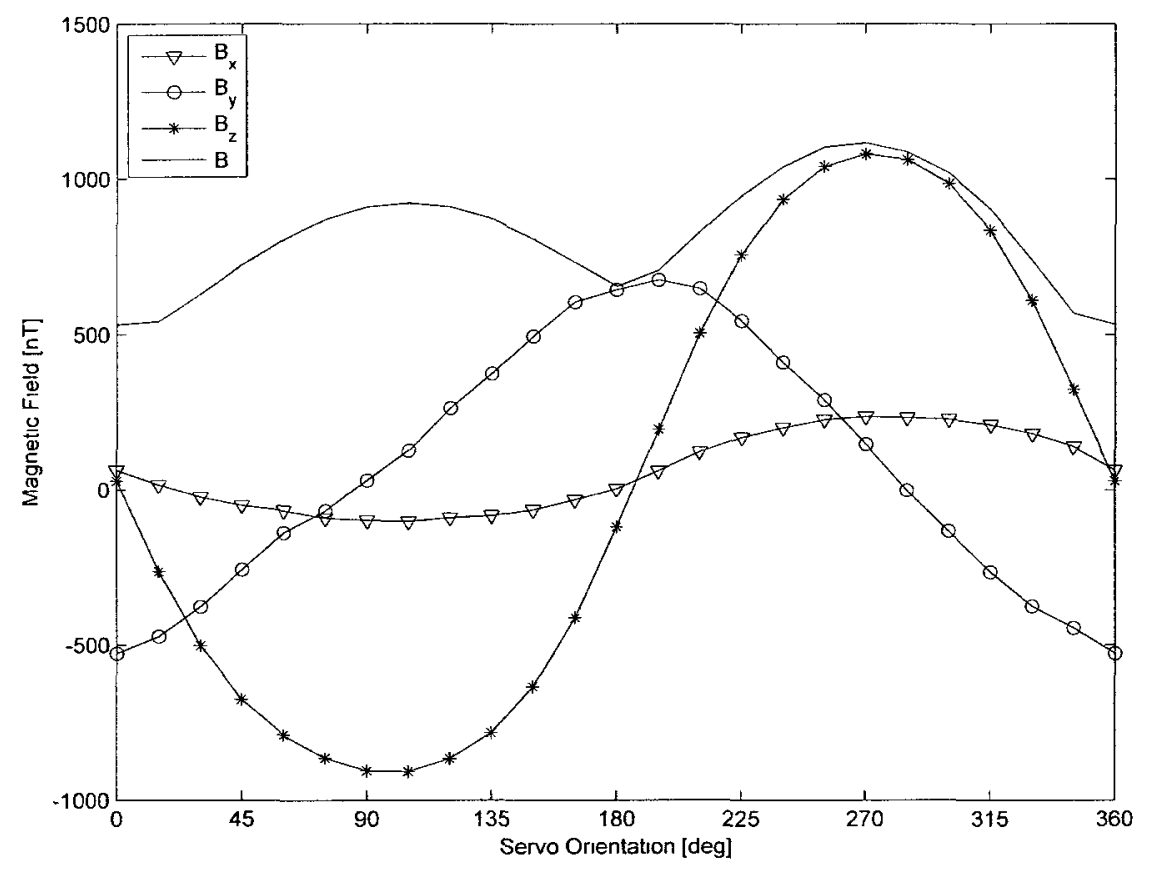

Figure A.4: Magnetic field vs. rotation for dual Hitec HSR-5990TG servos collected using MMTA-1D at a distance of $0.30 \mathrm{~m}$ in the $+\mathrm{z}$-direction of the measurement fluxgate. 


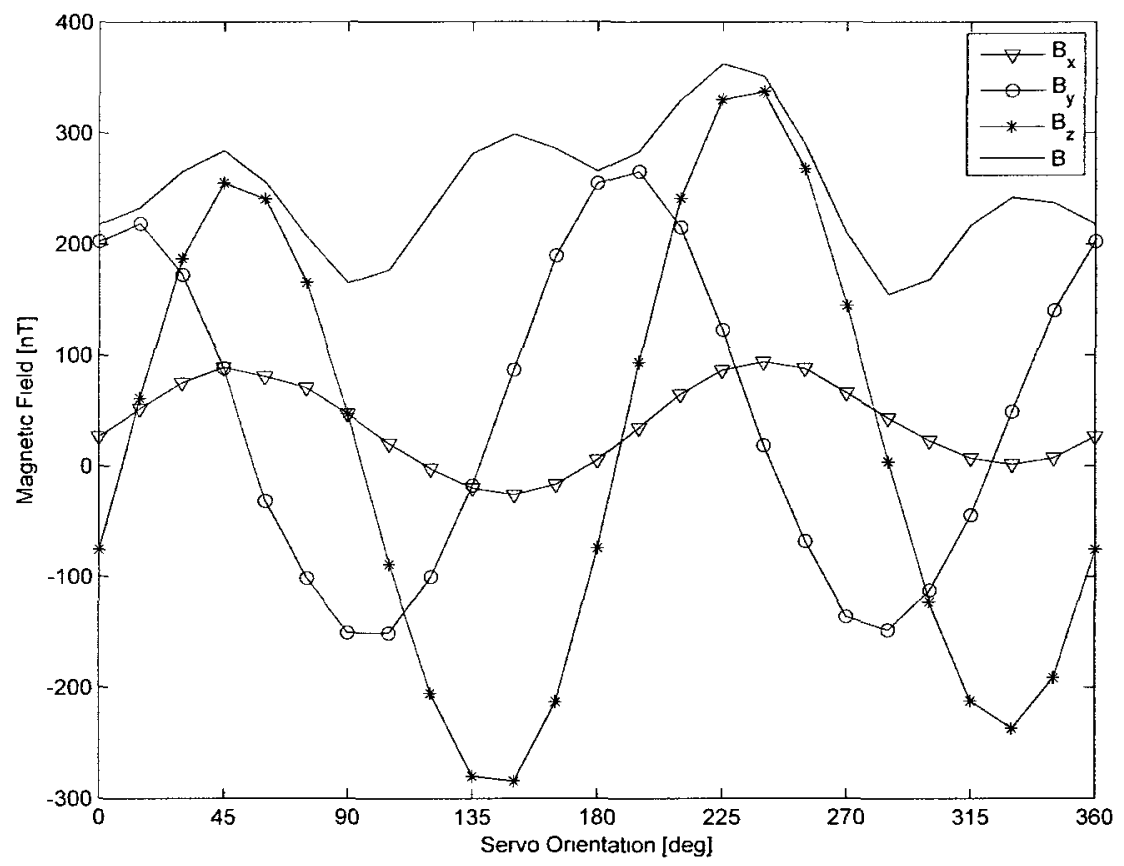

Figure A.5: Magnetic field vs. rotation for dual counteroriented Hitec HSR-5990TG servos collected using MMTA-1D at a distance of $0.30 \mathrm{~m}$ in the +z-direction of the measurement fluxgate. 

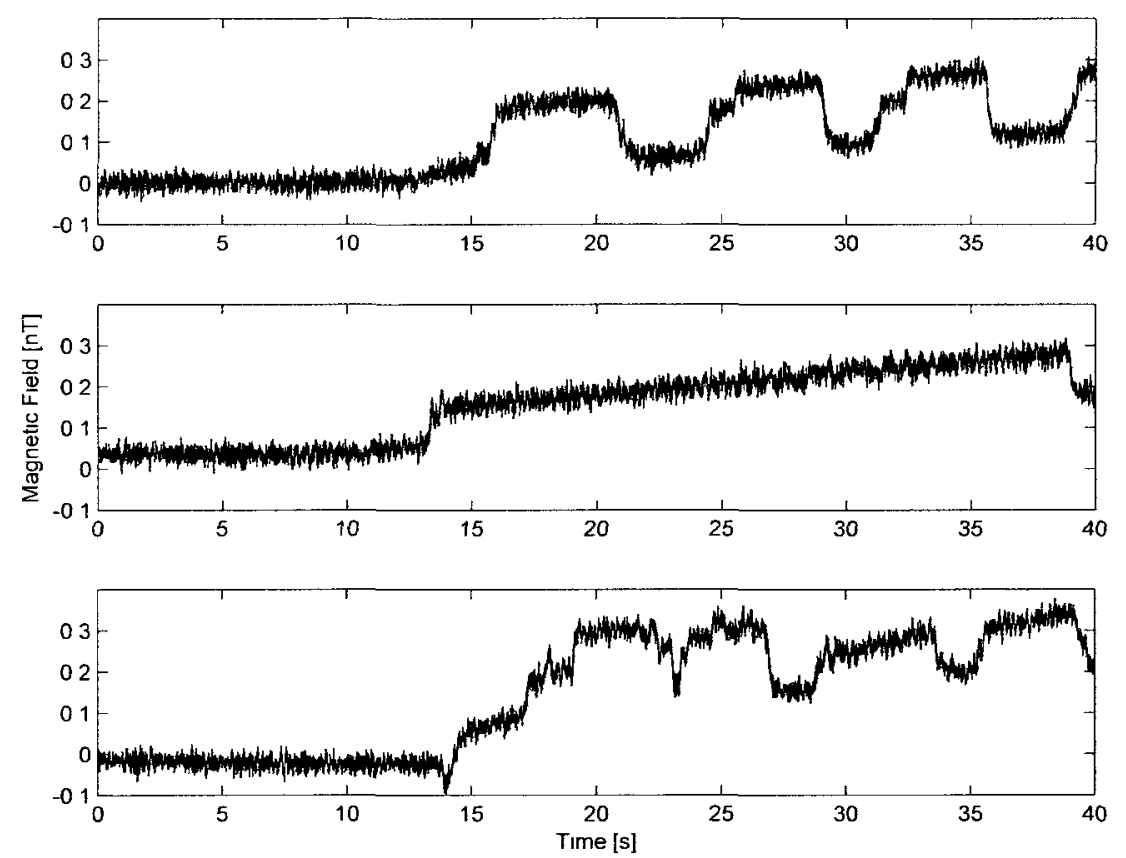

Figure A.6: Transient effect of cycling a Hitec HSR-5990TG servo at load of approximately $4.5 \mathrm{~kg} \cdot \mathrm{cm}$ at a distance of $0.691 \mathrm{~m}$. Shown are the results for cycling load/no load (top), constant load (middle), and the effect of the movement of the load alone (bottom). Sand bags were used for the load which had an adverse effect on the experimental results. 

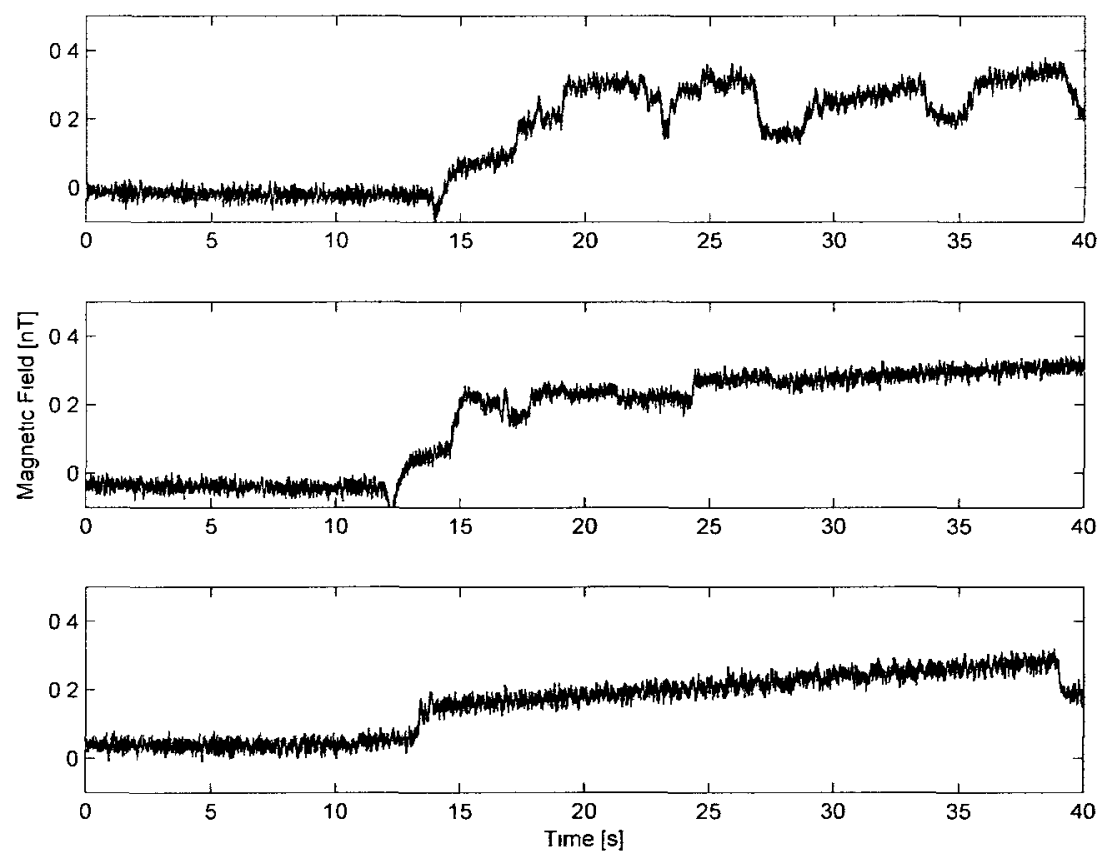

Figure A.7: Transient effect of cycling a Hitec HSR-5990TG servo at load of approximately $13.5 \mathrm{~kg} \cdot \mathrm{cm}$ at a distance of $0.691 \mathrm{~m}$. Shown are the results for cycling load/no load (top), constant load (middle), and the effect of the movement of the load alone (bottom). Sand bags were used for the load which had an adverse effect on the experimental results. 
Appendix B

Full-Scale UAS Testing Figures 

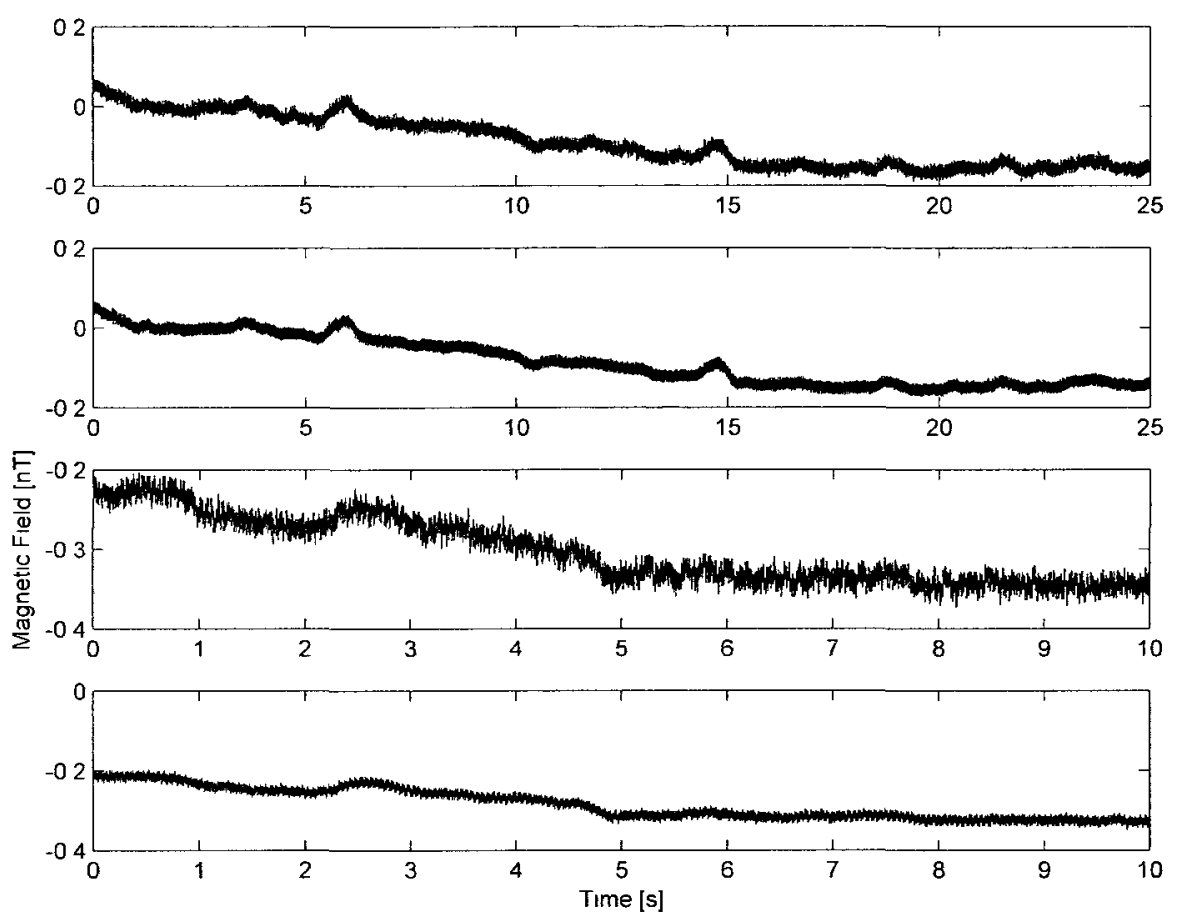

Figure B.1: Behaviour of magnetic field while actuating both elevators using powered servos. The magnetic noise effect for slow actuation at the starboard (top) and port (second from top) magnetometers, as well as fast actuation at the starboard (second from bottom) and port (bottom) magnetometers is shown. The magnetic noise observed at the wingtip magnetometers does not appear to be related to actuation of the elevator control surfaces.

NOTE: The large spikes in the Figures B.2 and B.3 were due to changes in the background field, and did not originate from the UAS itself. The variation in the background field can be distinguished from UAS generated sources by comparing both wingtip magnetometers. Since it was unlikely that the transient magnetic field effect was the same at both wingtips, it was concluded that spikes of identical amplitude at each wingtip sensor were due to variations in the background field. It is important to note that immediately prior to this series testing, a thunderstorm occurred leading to increased ionospheric activity causing the background magnetic field to be less stable. 

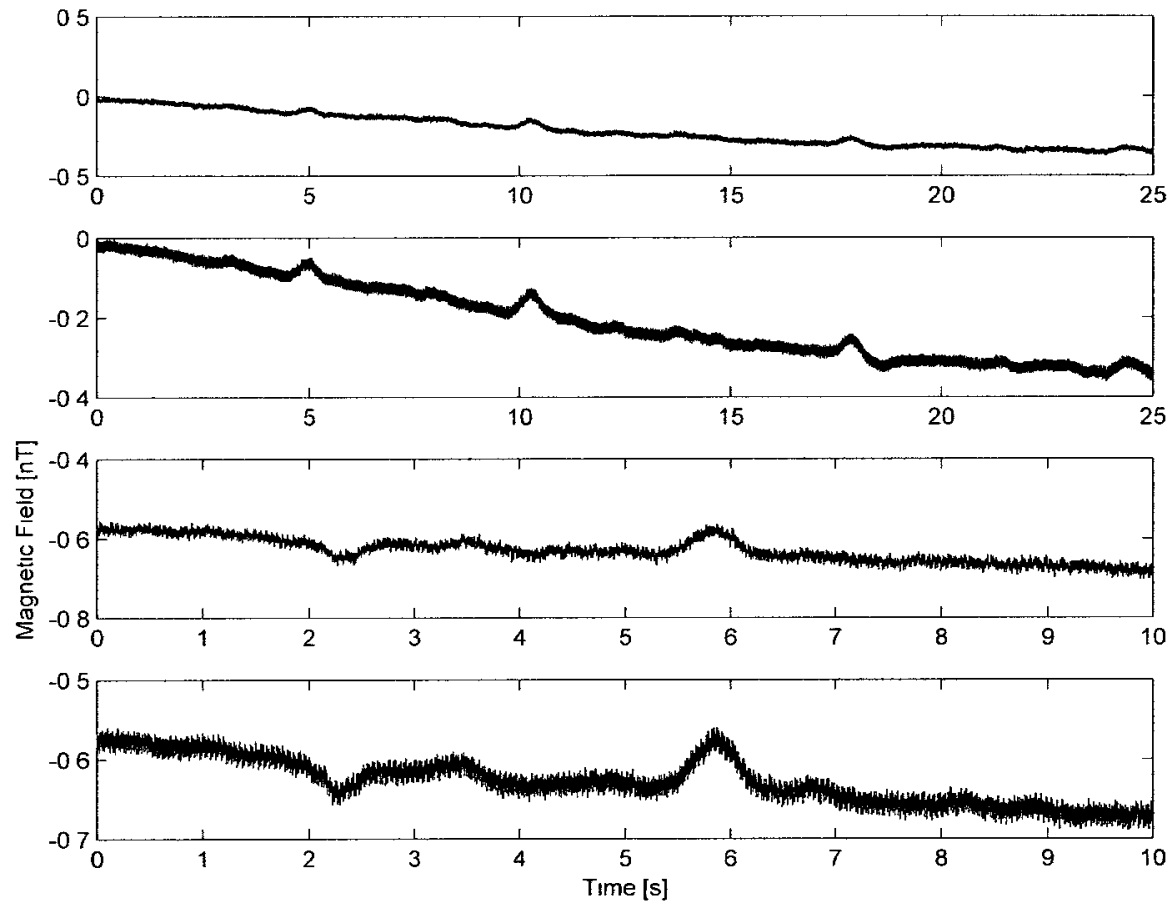

Figure B.2: Behaviour of magnetic field while actuating both rudders using powered servos. The magnetic noise cffect for slow actuation at the starboard (top) and port (second from top) magnetometers, as well as fast actuation at the starboard (second from bottom) and port (bottom) magnetometers is shown. The magnetic noise observed at the wingtip magnetometers does not appear to be related to actuation of the rudder control surfaces. 

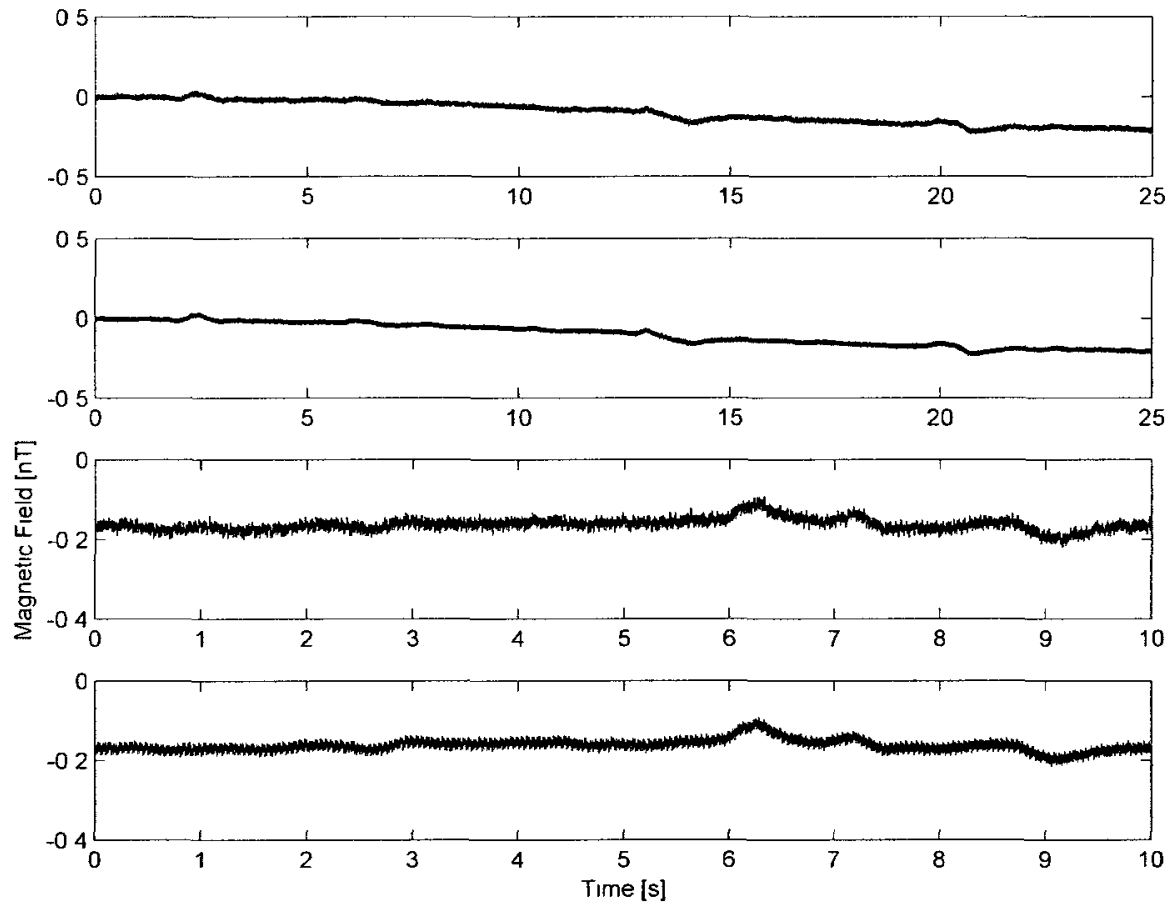

Figure B.3: Behaviour of magnetic field while actuating the throttle using a powered servo. The magnetic noise effect for slow actuation at the starboard (top) and port (second from top) magnetometers, as well as fast actuation at the starboard (second from bottom) and port (bottom) magnetometers is shown. The magnetic noise observed at the wingtip magnetometers does not appear to be related to actuation of the throttle. 
Appendix $\mathrm{C}$

GAMSO Efficiency Investigation 
Table C.1: Investigation of the number of generations required to reach the global minimum with GAMSO. The approximate elapsed time is shown for an Intel Core i5 $2.67 \mathrm{GHz}$ CPU. There were 65,536 unique solutions for this problem.

\begin{tabular}{|c|c|c|}
\hline Trial \# & Number of generations & Approximate Elapsed Time (hours) \\
\hline 1 & 7 & 0.47 \\
\hline 2 & 53 & 3.53 \\
\hline 3 & 7 & 0.47 \\
\hline 4 & 7 & 0.47 \\
\hline 5 & 13 & 0.87 \\
\hline 6 & 7 & 0.47 \\
\hline 7 & 7 & 0.47 \\
\hline 8 & 7 & 0.47 \\
\hline 9 & 21 & 1.4 \\
\hline 10 & 7 & 0.47 \\
\hline 11 & 120 & 8 \\
\hline 12 & 9 & 0.6 \\
\hline 13 & 7 & 0.47 \\
\hline 14 & 72 & 4.8 \\
\hline 15 & 278 & 18.5 \\
\hline 16 & 7 & 0.47 \\
\hline
\end{tabular}

